\title{
Strategies nurse educators use to integrate computer -assisted instruction into their courses to teach clinical decision-making
}

\author{
Beverly J. Schaefer \\ West Virginia University
}

Follow this and additional works at: https://researchrepository.wvu.edu/etd

\footnotetext{
Recommended Citation

Schaefer, Beverly J., "Strategies nurse educators use to integrate computer -assisted instruction into their courses to teach clinical decision-making" (2005). Graduate Theses, Dissertations, and Problem Reports. 2686.

https://researchrepository.wvu.edu/etd/2686

This Dissertation is protected by copyright and/or related rights. It has been brought to you by the The Research Repository @ WVU with permission from the rights-holder(s). You are free to use this Dissertation in any way that is permitted by the copyright and related rights legislation that applies to your use. For other uses you must obtain permission from the rights-holder(s) directly, unless additional rights are indicated by a Creative Commons license in the record and/ or on the work itself. This Dissertation has been accepted for inclusion in WVU Graduate Theses, Dissertations, and Problem Reports collection by an authorized administrator of The Research Repository @ WVU. For more information, please contact researchrepository@mail.wvu.edu.
} 
Strategies Nurse Educators Use to Integrate Computer-assisted Instruction Into their Courses to Teach Clinical Decision-making

Beverly J. Schaefer

Dissertation submitted to the College of Human

Resources and Education at West Virginia University in partial fulfillment of the requirements for the degree of

\author{
Doctor of Education \\ in \\ Technology Education \\ David McCrory, Ph.D., Chair \\ Norman Lass, Ph.D. \\ Dorothy Johnson, Ed. D. \\ Neal Shambaugh, Ph.D. \\ Lawrence Stead, Ph.D. \\ Department of Advance Educational Studies
}

Morgantown, West Virginia

2005

Keywords: CAI, Clinical Decision-making, Computer-assisted Instruction, Electronic On-line Questionnaire, Integration of Instruction, Integration Strategies, Instructional Design, Nursing Education, Software, Software for Nursing Education 


\begin{abstract}
Strategies Nurse Educators Use to Integrate Computer-assisted Instruction Into their Courses to Teach Clinical Decision-making
\end{abstract}

Beverly J. Schaefer

The strategies nurse educators' use to integrate computer-assisted instruction (CAI) designed to teach clinical decision-making into their courses were investigated to determine their frequency of use and effectiveness. The "Integration of CAI Questionnaire," an on-line electronic survey designed by the investigator, was used to collect data. A stratified randomlyselected group of 109 schools of nursing that was a statistically representative mix of baccalaureate (BSN), associate (AD) and diploma schools from each of six geographic regions of the United States comprised the sample.

The study questions were as follows: (1) What integration strategies have nurse educators used to integrate CAI? (2) How effective did participants perceive the strategies were for increasing student learning, decreasing course costs, students' time and the teachers' time? (3) What were the key features of the schools and educators that integrated CAI effectively? (4) What types of software did nurse educators use to teach clinical decision-making? (5) How effective were their peers at integrating the software and how effectively did they integrate commercially-purchased and in-house produced software?

Statistical analysis consisted of measures of central tendency, frequencies, and percentages. The Pearson $r$ was used to determine the relationships between the frequency of use and perceptions of the effectiveness of the strategies.

The findings showed that $71 \%$ of the schools used CAI and that more AD (81\%) and diploma schools $(80 \%)$ used CAI than BSN (56\%). In fact, AD schools and teachers used CAI more on most measures and rated their peers as more "effective" at integrating CAI than their BSN counterparts. Twenty-nine of the 44 strategies were "frequently" used and 17 were perceived to be "effective." Significant correlations $(\mathrm{p}<0.01)$ were noted for "explain CAI, explain objectives, motivate, integrate, individual mode, course exam and follow-up." "Technical" and "instructional" support were the two most "frequently" used and "effective" strategies for increasing learning and decreasing students' and teachers' time, but not costs. BSN schools used more strategies "frequently" but almost no differences in AD and BSN participants" perceptions of "effectiveness" were noted. Tutorials and drill-and-practice programs were used most and $95 \%$ of the programs were commercially-purchased. 


\section{DEDICATION}

I would like to dedicate this research to my mother, Julia C. Tyler, who made it possible in so many ways. It is also dedicated to the nurse educators across the United States and particularly the ones who participated in the study and expressed so much interest in learning about the results. It is hoped that the findings of this study will be useful to teachers in all areas of education as they strive to improve their classroom practices and seek to design their courses so that all four of the measures of educational productivity are improved. 


\section{ACKNOWLEDGMENTS}

I would like to acknowledge the following individuals for aiding me in the completion of my dissertation research. To my dissertation committee: Professors Dr. Norman Lass, Dr. Dorothy Johnson, Dr. Neal Shambaugh, Dr. Floyd L. Stead and my chairperson, Dr. David McCrory. I am also grateful to Dr. Ernest Goers who so graciously filled in at the last moment for Dr. Stead at my defense and contributed so much to the discussion. I would also like to thank Dr. George Maughan who assisted me in the early stages of its development. A special thanks to Dr. Johnson who went so far above and beyond what I would have expected by attending my defense. Thank you all for the time you spent in helping me in the preparation of this document. It was a privilege for me to have studied at West Virginia University in the Department of Technology Education and the College of Human Resources and Education.

I would also like to express my gratitude to my family and, in particular my husband, Richard for the support and encouragement he has offered during the entire process and my two children Corby and Scott for their belief in me and their encouragement over the years. 


\section{TABLE OF CONTENTS}

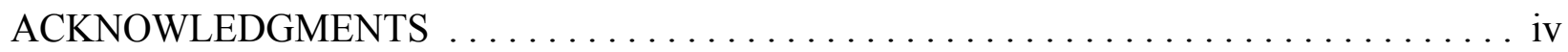

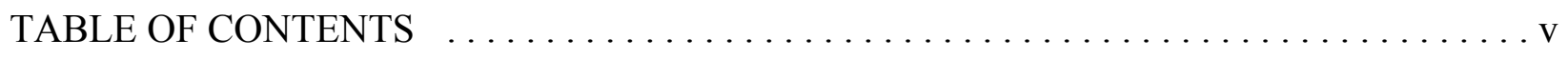

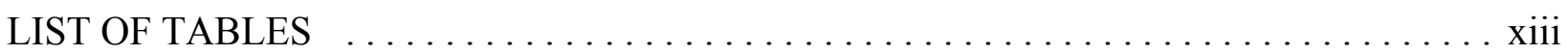

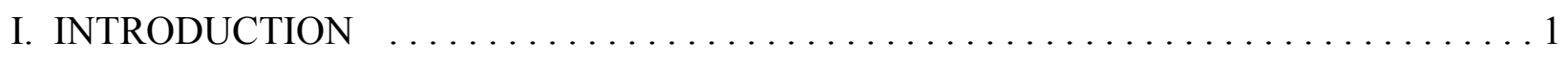

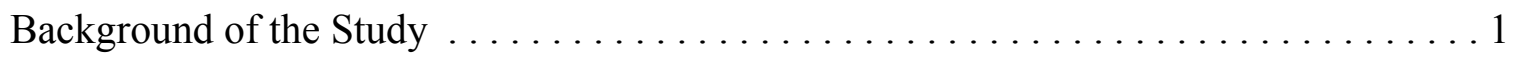

Clinical Decision-making Is the Backbone of Nursing $\ldots \ldots \ldots \ldots \ldots \ldots 1$

Innovative Teaching Methods $\ldots \ldots \ldots \ldots \ldots \ldots \ldots \ldots \ldots \ldots \ldots \ldots \ldots$

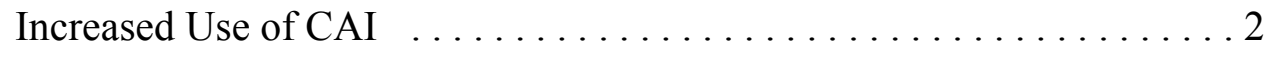

Nursing Educations' Unique Position $\ldots \ldots \ldots \ldots \ldots \ldots \ldots \ldots$

Integration Skills of Teachers $\ldots \ldots \ldots \ldots \ldots \ldots \ldots \ldots \ldots$

Complexity of the Task Underestimated $\ldots \ldots \ldots \ldots \ldots \ldots \ldots$

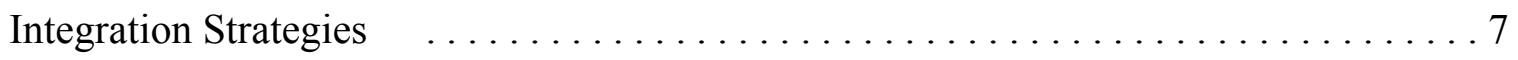

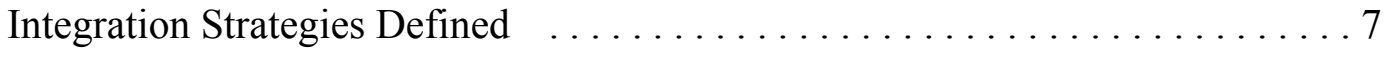

Educational Productivity $\ldots \ldots \ldots \ldots \ldots \ldots \ldots \ldots \ldots \ldots \ldots \ldots$

Strategies to Increase Learning $\ldots \ldots \ldots \ldots \ldots \ldots \ldots$

Strategies to Decrease the Students' Time $\ldots \ldots \ldots \ldots \ldots \ldots \ldots$

Strategies to Decrease Costs $\ldots \ldots \ldots \ldots \ldots \ldots \ldots \ldots \ldots \ldots \ldots$

Strategies to Decrease the Teachers' Time $\ldots \ldots \ldots \ldots \ldots \ldots \ldots$

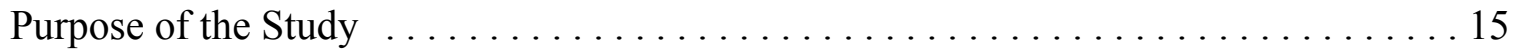




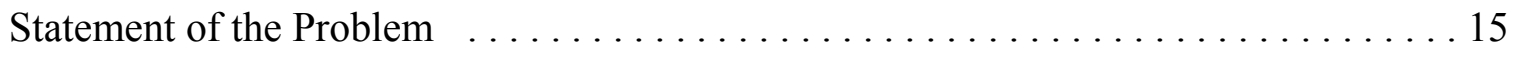

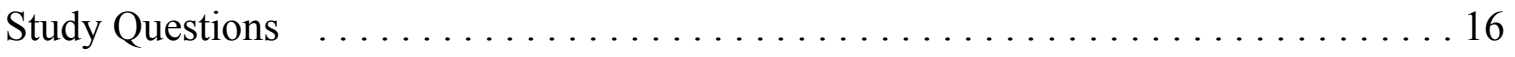

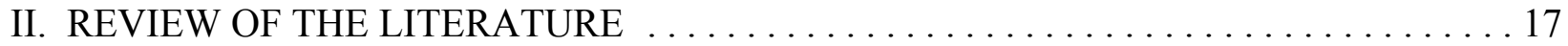

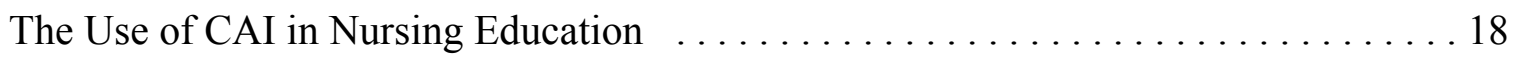

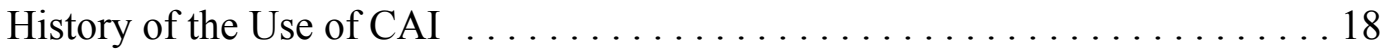

Measures of Educators' Skills $\ldots \ldots \ldots \ldots \ldots \ldots \ldots$

Interventions to Improve Educators' Skills $\ldots \ldots \ldots \ldots \ldots \ldots \ldots 21$

Use of CAI in General Education $\ldots \ldots \ldots \ldots \ldots \ldots \ldots \ldots \ldots \ldots \ldots \ldots$

CAI vs. Traditional Teaching Methods $\ldots \ldots \ldots \ldots \ldots \ldots \ldots . \ldots 23$

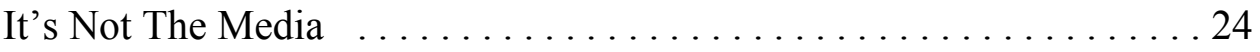

Effectiveness of CAI for Nursing Education $\ldots \ldots \ldots \ldots \ldots \ldots \ldots \ldots$

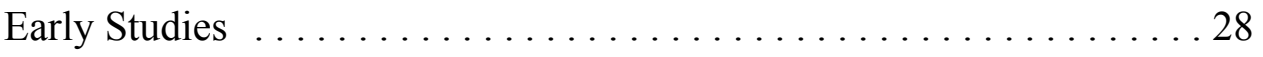

Quality of the Research Questioned .................... 29

Uses of CAI to Teach Clinical Decision-making $\ldots \ldots \ldots \ldots \ldots \ldots \ldots \ldots \ldots \ldots$

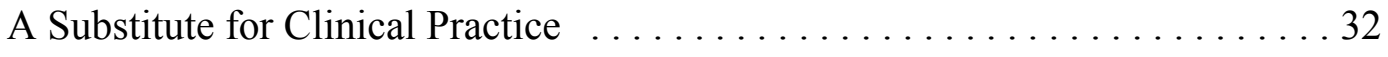

Expect Different Learning Outcomes $\ldots \ldots \ldots \ldots \ldots \ldots \ldots \ldots \ldots$

Expert Systems Support Clinical Decision-making $\ldots \ldots \ldots \ldots . \ldots 37$

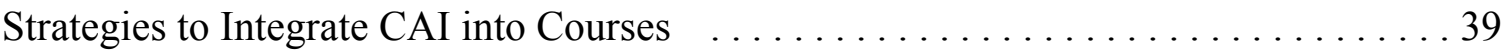

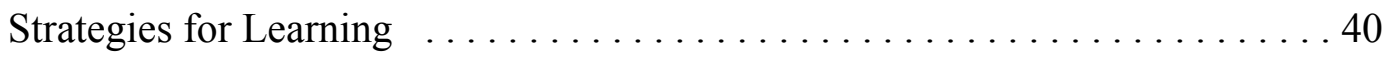

Preinstruction strategies $\ldots \ldots \ldots \ldots \ldots \ldots \ldots \ldots \ldots \ldots \ldots \ldots$

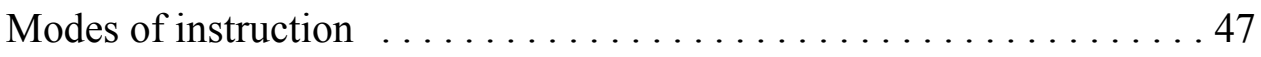

Strategies to Decrease the Students' Time ...................... 53 


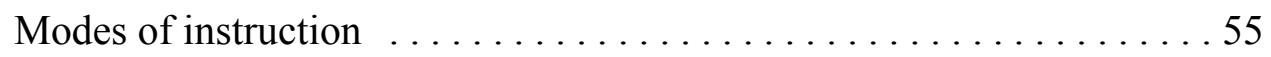

Technical and instructional support $\ldots \ldots \ldots \ldots \ldots \ldots \ldots 62$

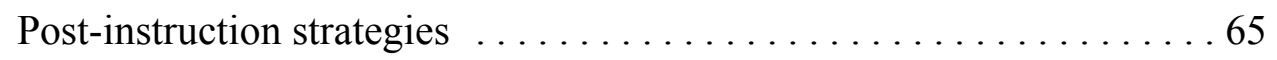

Strategies to Decrease Costs $\ldots \ldots \ldots \ldots \ldots \ldots \ldots \ldots \ldots \ldots \ldots \ldots$

Replace traditional instruction . . . . . . . . . . . . . . . . 89 89

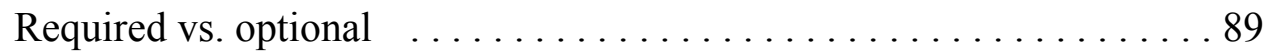

Modes of instruction $\ldots \ldots \ldots \ldots \ldots \ldots \ldots \ldots \ldots \ldots$

Technical and instructional support $\ldots \ldots \ldots \ldots \ldots \ldots \ldots . \ldots 9$

Strategies to Decrease the Teachers' Time .................... 104

Replace traditional instruction $\ldots \ldots \ldots \ldots \ldots \ldots \ldots . \ldots \ldots . \ldots . \ldots 104$

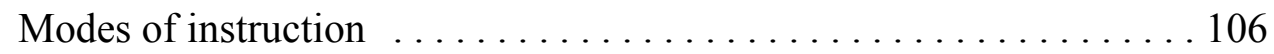

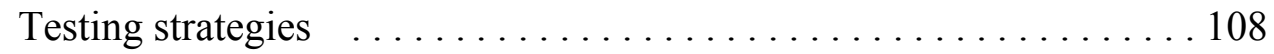

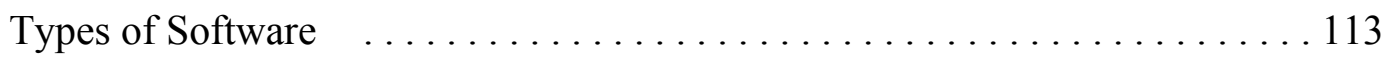

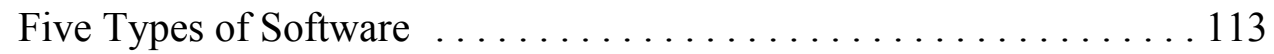

Commercially and In-house Produced Software $\ldots \ldots \ldots \ldots \ldots 114$

Key Features of Schools and Faculty That Effectively Integrate CAI . . . . . . . 120

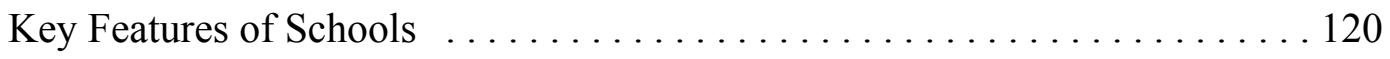

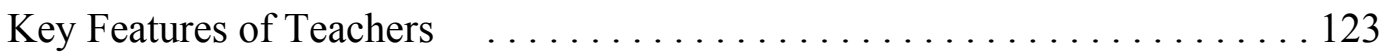

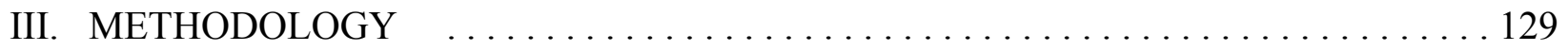

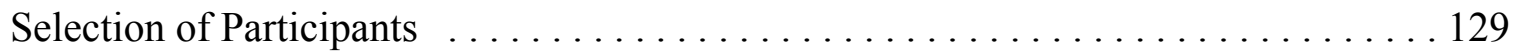

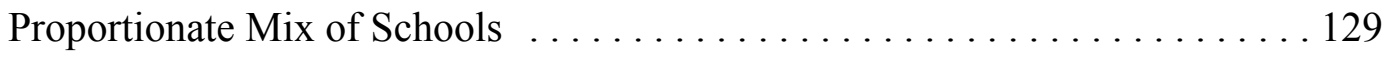

Proportionate Mix of Geographic Regions .................... 129 


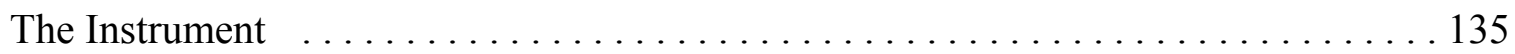

The Integration of Computer Assisted Instruction Questionnaire . . . . . . 135

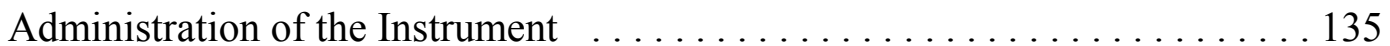

Validity and Reliability of the Instrument $\ldots \ldots \ldots \ldots \ldots \ldots \ldots$

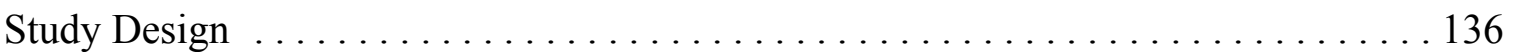

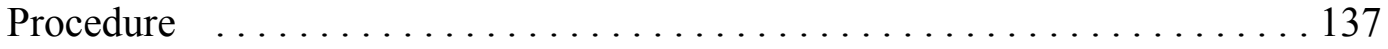

Analysis of the Data ... . . . . . . . . . . . . . . . . . . . . 139

Assumptions Underlying the Study $\quad \ldots . \ldots \ldots \ldots \ldots \ldots . \ldots \ldots . \ldots \ldots$

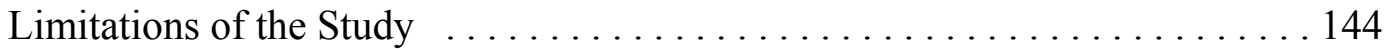

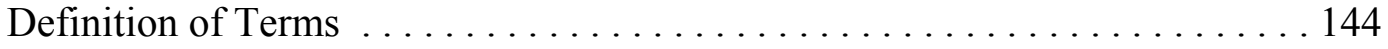

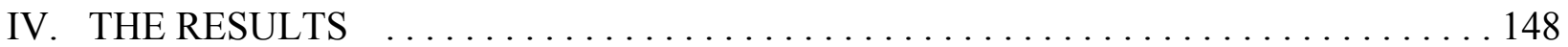

The Sample . . . . . . . . . . . . . . . . . . . . . . . . . . 149

Sample Schools ........................ 151

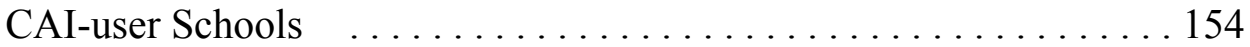

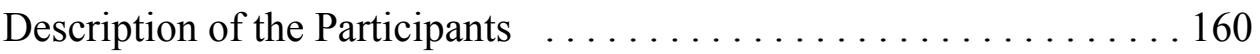

A Non-respondent Bias-check $\quad \ldots \ldots \ldots \ldots \ldots 16 \ldots \ldots \ldots \ldots$

Study Question 1 Findings - Frequency $\ldots \ldots \ldots \ldots \ldots$............. 163

Study Question 2 Findings - Effectiveness $\ldots \ldots \ldots \ldots \ldots \ldots$

Strategies to Increase Learning $\ldots \ldots \ldots \ldots \ldots \ldots \ldots \ldots$

Strategies to Decrease Costs . . . . . . . . . . . . . . . 173

Strategies for Decreasing the Students' Time . . . . . . . . . . . . . . . . . 174

Strategies to Decrease the Teachers' Time . . . . . . . . . . . 175

viii 
Correlation Findings $\ldots \ldots \ldots \ldots \ldots \ldots \ldots \ldots \ldots \ldots \ldots \ldots$

Study Question 3 Finding - Key Features of Schools $\ldots \ldots \ldots \ldots \ldots \ldots 182$

Comparison of the Frequency of Use $\ldots \ldots \ldots \ldots \ldots \ldots \ldots . \ldots 183$

Comparison of Effectiveness of Strategies for Learning ........ 191

Comparison of Effectiveness of Strategies for Costs _......... 197

Comparison of Effectiveness of Strategies for Students' Time . . . . 199

Comparison of Effectiveness of Strategies to Decrease Teachers' Time

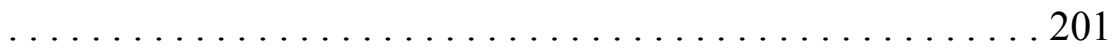

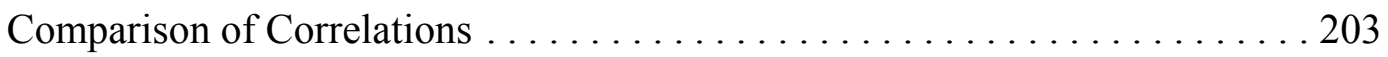

Comparison of Correlations for Learning .................. 204

Comparison of Correlations for Costs $\ldots \ldots \ldots \ldots \ldots \ldots \ldots 206$

Comparison of Correlations for Students' Time . . . . . . . . . . . 207

Comparison of Correlations for Teachers' Time $\ldots \ldots \ldots \ldots \ldots 208$

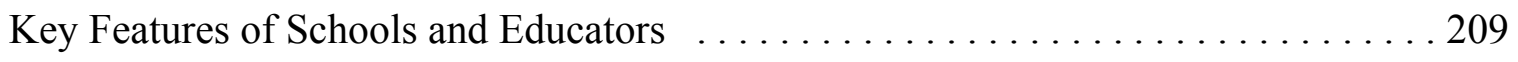

Key Features of Schools .............................. 209

Schools with a Computer Lab in the SON $\ldots \ldots \ldots \ldots \ldots . \ldots 209$

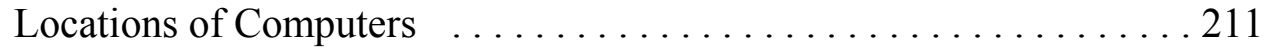

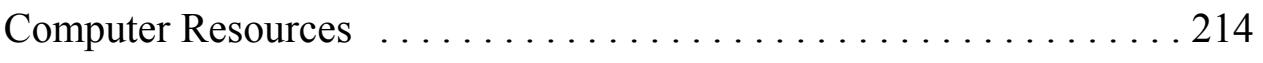

Technical and Instructional Support Personnel $\ldots \ldots \ldots \ldots \ldots .217$

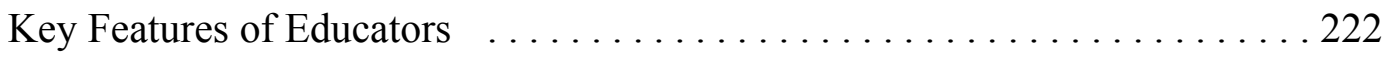

Key Features of Participants . . . . . . . . . . . . . . . . 227

Key Features of Schools That Integrate Effectively $\ldots \ldots \ldots \ldots 235$

Study Question 4 Findings- Types of Software $\ldots \ldots \ldots \ldots \ldots \ldots \ldots . \ldots 247$ 


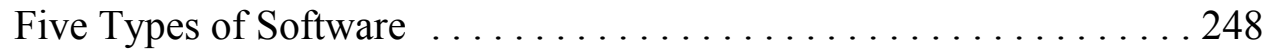

Commercially Produced and In-house Produced Software ....... 251

Study Question 5 Finding- Ratings of Peer's Effectiveness $\ldots \ldots \ldots \ldots 253$

Peer Ratings of Effectiveness for Integrating 5 Types of Software $\quad$. 254

Peer Rating of Effectiveness of Commercial and In-house Produced . . 257

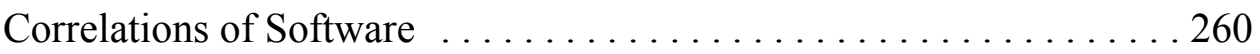

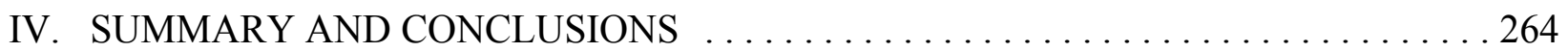

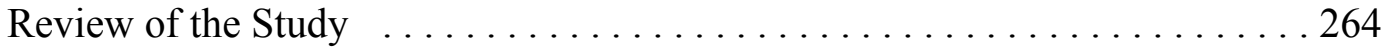

The Problem Statement $\ldots \ldots \ldots \ldots \ldots \ldots \ldots \ldots \ldots . \ldots \ldots$

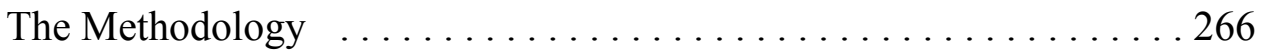

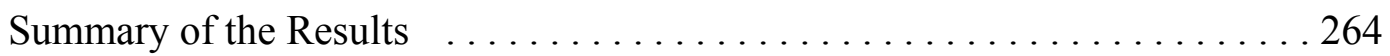

Description of the Sample $\ldots \ldots \ldots \ldots \ldots \ldots \ldots \ldots \ldots . \ldots . \ldots 266$

Discussion of the findings $\ldots \ldots \ldots \ldots \ldots \ldots \ldots \ldots \ldots$

Frequently used strategies $\ldots \ldots \ldots \ldots \ldots \ldots \ldots \ldots 268$

Effective strategies $\ldots \ldots \ldots \ldots \ldots \ldots \ldots . \ldots . \ldots . \ldots 269$

Significant correlations $\ldots \ldots \ldots \ldots \ldots \ldots \ldots \ldots \ldots 270$

Comparison of strategies $\ldots \ldots \ldots \ldots \ldots \ldots \ldots . \ldots 271$

Computer resources $\ldots \ldots \ldots \ldots \ldots \ldots \ldots \ldots \ldots \ldots \ldots$

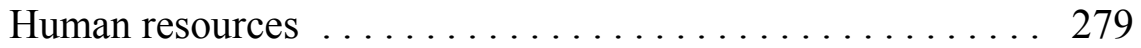

Key features of effective schools $\ldots \ldots \ldots \ldots \ldots \ldots 284$ 
Effectiveness of the Strategies for Learning . . . . . . . . . . . 302

Effectiveness of Strategies for Decreasing Costs $\ldots \ldots \ldots \ldots \ldots . \ldots 306$

Effectiveness of Strategies to Decrease Students' Time . . . . . . . 308

Effectiveness of Strategies to Decrease the Teachers' Time ...... 309

Key Features of Effective Schools and Educators $\ldots \ldots \ldots \ldots \ldots \ldots 313$

Comparisons of Effectiveness of Strategies for Learning ........ 314

Comparison of Effectiveness of Strategies to Decrease Costs ..... 316

Comparison of Effectiveness to Decrease Students' Time ........ 317

Comparison of Effectiveness to Decrease Teachers' Time ....... 318

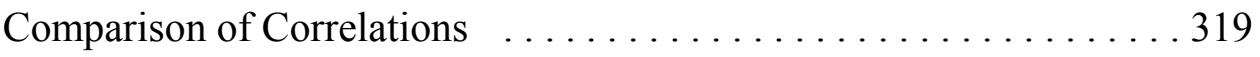

Key Features of Schools ............................... 321

Schools With a Lab in the SON ........................ 321

Locations of Computers ............................. 321

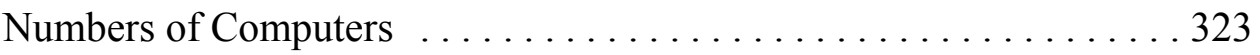

Technical and Instructional Support $\ldots \ldots \ldots \ldots \ldots \ldots \ldots \ldots \ldots$

Key Features of Effective Educators $\ldots \ldots \ldots \ldots \ldots \ldots \ldots \ldots \ldots$

Less Concrete, but Interesting Key Features of Schools ........ 330

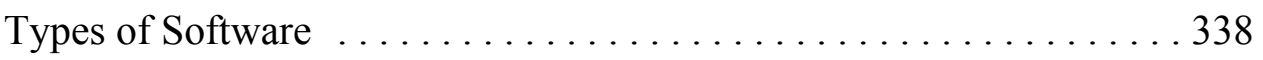

Peers' Ratings of Effectiveness $\ldots \ldots \ldots \ldots \ldots \ldots \ldots \ldots \ldots$.............. 341

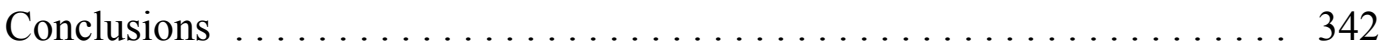

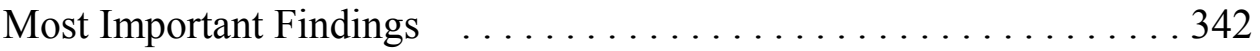


Unanticipated Occurrences $\ldots \ldots \ldots \ldots \ldots \ldots \ldots \ldots \ldots \ldots \ldots \ldots$

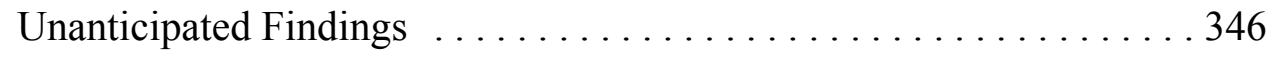

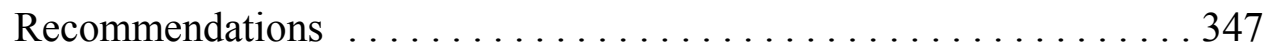

Recommendations for Nursing Education $\ldots \ldots \ldots \ldots 347$

Recommendations for General Education $\ldots \ldots \ldots \ldots 349$

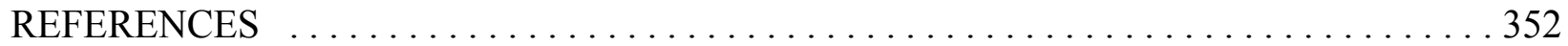

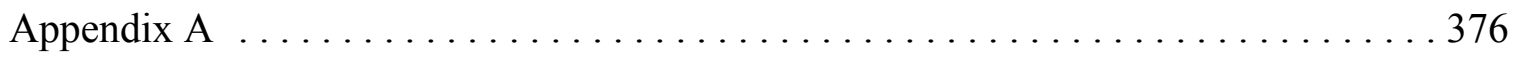

Appendix B ............................................... 389

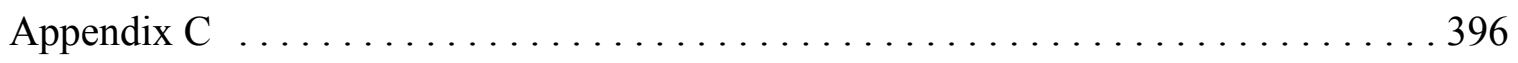

Appendix D ....................................... 464

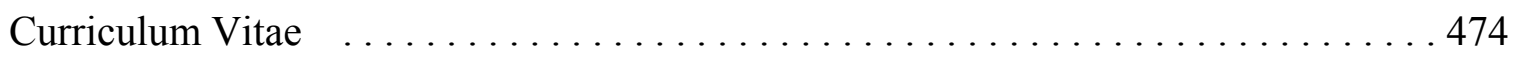




\section{LIST OF TABLES}

Table 1. Percent and Number of Each Type of School in the Population and the Sample . . . 130

Table 2. Randomized Selection of Schools from Six Geographic Divisions . . . . . . . . . 130

Table 3. Summary of Study Questions, Questionnaire Questions Answered and Methods of

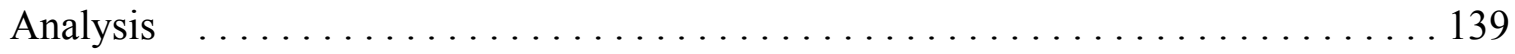

Table 4. Comparison of Proportionate Mix of Schools in the Population and the Sample . . 149

Table 5. Comparison of Intended Target and Actual Number of Schools from Each Geographic

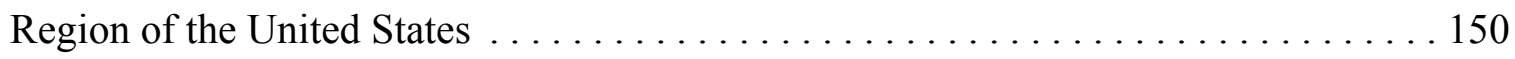

Table 6. Student Enrollments: Number of Students and Average Enrollments in Public and

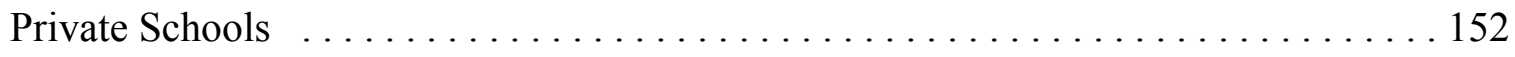

Table 7. Division of Sample Schools into Three Groups based on Student Enrollments in the

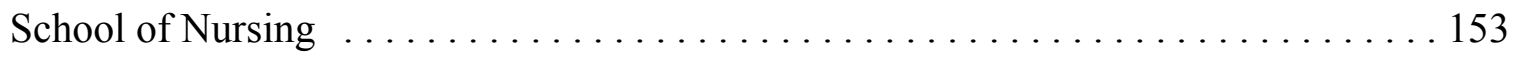

Table 8. CAI-user Schools and Non-users in the Sample by Type of School: Responses to Question One of the ICAI Questionnaire $\ldots \ldots \ldots \ldots \ldots \ldots \ldots \ldots \ldots \ldots \ldots \ldots \ldots \ldots \ldots \ldots \ldots \ldots$

Table 9. Percent of all Bachelor, Associate and Diploma Schools that Use CAI: Response to

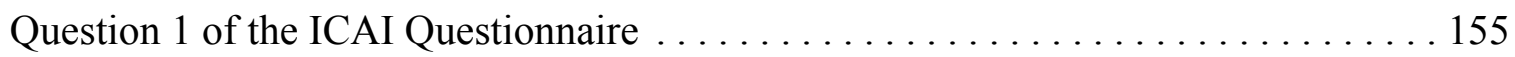

Table 10. CAI-user Schools and Non-user Schools in Sample based on Type of Institution According to Responses to Question 1 of the ICAI Questionnaire . . . . . . . . 156

Table 11. Percent of Schools that are CAI-users and Non-users based on School Size: Responses to Question 1 of the ICAI Questionnaire $\ldots \ldots \ldots \ldots \ldots \ldots \ldots \ldots \ldots \ldots \ldots \ldots \ldots \ldots \ldots$ 
Table 12. Percent of CAI-user Schools by Size and Type of School According to Responses to

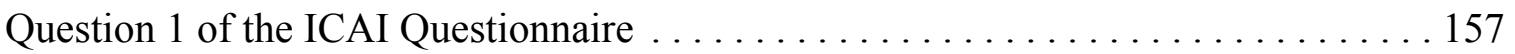

Table 13. Means of Frequency of Use and Perceived Effectiveness of Integration Strategies for Increasing Student Learning, Decreasing Costs, Students’ Time and Teachers' Time

Table 14. Correlations Between Frequency of Use and Perceived Effectiveness of the

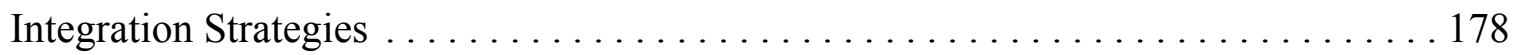

Table 15. Integration Strategies That Were Frequently Used and Perceived to be Effective for Increasing Student Learning by BSN and AD Schools

Table 16. Measures of Central Tendency of Number of Teachers That Use CAI in Response to Question 18 A by Type of School, Type of Institution, Size of School 224

Table 17. Key Features of Schools: Comparison of the Means of the Integration Strategies . 239 Table 18. Percent of Educators Who Use CAI: Comparison of the Means of the Integration

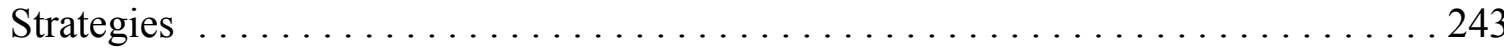

Table 19. Correlations between Frequency of Use and Ratings of Peer's Effectiveness at Integrating the Five Types of Software based on School Sizes . . . . . . . . . . . . 261

Table B 1. CAI-user and Non-User Schools: Non-Response Bias-check . . . . . . . . . . . . 389

Table B 2. Types of Schools and Institutions in Sample and Non-respondent Group: NonResponse Bias-check ................................. 390

Table B 3. Average Student Enrollments in Sample and Non-respondent Groups: Bias-check

Table B 4. Schools with a Computer Lab in the School of Nursing: Bias-check . . . . . . . . . 392 
Table B 5. Participants' Roles: Non-Response Bias-check . . . . . . . . . . . . . . . . . . 393

Table B 6. Participants' Genders: Non-Response Bias-check . . . . . . . . . . . . . . . 393

Table B 7. Participants Age Groups: Non-Response Bias-check . . . . . . . . . . . . . . . 394

Table B 8. Participants' Educational Backgrounds: Non-Response Bias-check . . . . . . . 395

Table C 1. Frequency of Use of Integration Strategies: Measures of Central Tendency . . . . 396

Table C 2. Percentages of Frequency of Use of Integration Strategies . . . . . . . . . . . 397

Table C 3. Effectiveness of Integration Strategies: Measures of Central Tendency . . . . . 398

Table C 4. Percentages of Perceived Effectiveness of Integration Strategies to Increase Student

Learning ................................................... 399

Table C 5. Perceived Effectiveness of Integration Strategies for Decreasing Costs: Measures of

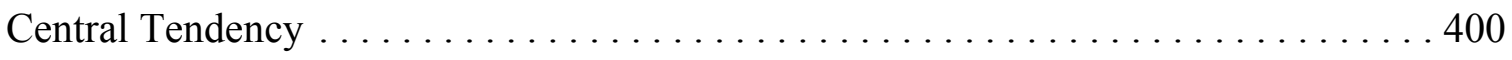

Table C 6. Percentages of Perceived Effectiveness of Integration Strategies for Decreasing

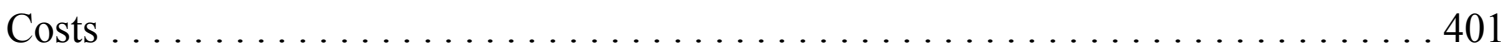

Table C 7. Perceived Effectiveness of Integration Strategies for Decreasing Students' Time:

Measures of Central Tendency $\ldots \ldots \ldots \ldots \ldots \ldots \ldots \ldots \ldots \ldots \ldots . \ldots . \ldots . \ldots . \ldots 2$

Table C 8. Percentages of Perceived Effectiveness of Integration Strategies to Decrease

Students' Time

Table C 9. Perceived Effectiveness of Integration Strategies for Decreasing Teachers' Time: Measures of Central Tendency $\ldots \ldots \ldots \ldots \ldots \ldots \ldots \ldots \ldots \ldots . \ldots . \ldots . \ldots 4$

Table C 10. Percentages of Perceived Effectiveness of Integration Strategies to Decrease

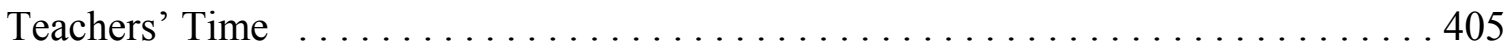


Table C 11. Comparison of Means of Frequency of Use of Integration Strategies by Type of School 406

Table C 12. Comparison of Frequency of Use of Integration Strategies based on Type of Institution 407

Table C 13. Comparison of Frequency of Use of Integration Strategies based on School

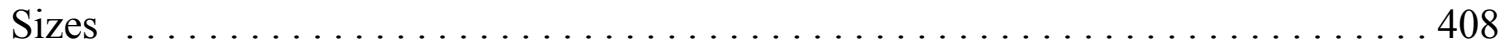

Table C 14. Comparison of Perceived Effectiveness of Integration Strategies to Increase Student

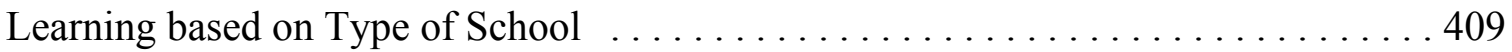

Table C 15. Comparison of the Perceived Effectiveness of the Integration Strategies to Increase Student Learning based on Type of Institution $\ldots \ldots \ldots \ldots \ldots \ldots \ldots \ldots \ldots \ldots$

Table C 16. Comparison of the Perceived Effectiveness of Integration Strategies to Increase Student Learning based on School Size $\ldots \ldots \ldots \ldots \ldots \ldots \ldots \ldots \ldots \ldots \ldots \ldots$

Table C 17. Comparison of Perceived Effectiveness of Integration Strategies for Decreasing Costs based on Type of School . . . . . . . . . . . . . . . . . . . . 412

Table C 18. Comparison of Perceived Effectiveness of Integration Strategies for Decreasing Costs based on Type of Institution

Table C 19. Comparison of Perceived Effectiveness of Integration Strategies for Decreasing Costs based on School Sizes 414

Table C 20. Comparison of Perceived Effectiveness of Integration Strategies for Decreasing Students' Time based on Type of School $\ldots \ldots \ldots \ldots \ldots \ldots \ldots \ldots \ldots \ldots$ Table C 21. Comparison of Perceived Effectiveness of Integration Strategies for Decreasing Students' Time based on Type of Institution 416 
Table C 22. Comparison of Perceived Effectiveness of Integration Strategies for Decreasing Students' Time based on Size of Schools $\ldots \ldots \ldots \ldots \ldots \ldots \ldots \ldots \ldots \ldots . \ldots 417$

Table C 23. Comparison of Perceived Effectiveness of Integration Strategies for Decreasing Teachers' Time based on Type of School $\ldots \ldots \ldots \ldots \ldots \ldots \ldots \ldots \ldots \ldots . \ldots 418$

Table C 24. Comparison of Perceived Effectiveness of Integration Strategies for Decreasing Teachers' Time based on Type of Institution $\ldots \ldots \ldots \ldots \ldots \ldots \ldots \ldots \ldots . \ldots 419$

Table C 25. Comparison of Perceived Effectiveness of Integration Strategies for Decreasing Teachers' Time based on Size of School . . . . . . . . . . . . . . . . . . 420

Table C 26. Comparison of Correlations Between Frequency of Use and Perceived Effectiveness of Integration Strategies for Increasing Student Learning by Type of School

Table C 27. Comparison of Correlations Between Frequency of Use and Perceived Effectiveness of Integration Strategies for Increasing Student Learning by Type of

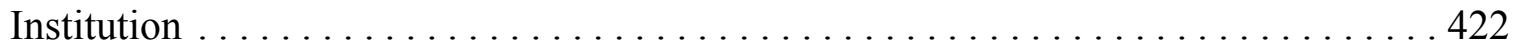

Table C 28. Comparison of Correlations Between Frequency of Use and Perceived Effectiveness of Integration Strategies for Increasing Student Learning by School Size . .

Table C 29. Correlation Between Frequency of Use and Perceived Effectiveness of Strategies to Decrease Costs by Type of School 424

Table C 30. Correlation Between Frequency of Use and Perceived Effectiveness of Strategies to Decrease Costs by Type of Institution 425 
Table C 31. Correlation Between Frequency of Use and Perceived Effectiveness of Strategies to

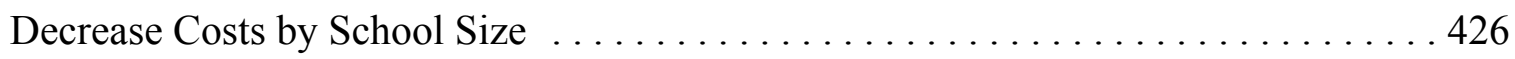

Table C 32. Correlation Between Frequency of Use and Perceived Effectiveness of Strategies to

Decrease the Students' Time by Type of School . . . . . . . . . . . . . 427

Table C 33. Correlation Between Frequency of Use and Perceived Effectiveness of Strategies to Decrease the Students' Time by Type of Institution $\ldots \ldots \ldots \ldots \ldots \ldots \ldots \ldots 28$

Table C 34. Correlation Between Frequency of Use and Perceived Effectiveness of Strategies to Decrease the Students' Time by School Size . . . . . . . . . . . . . . . . . . 429

Table C 35. Correlation Between Frequency of Use and Perceived Effectiveness of Strategies to Decrease the Teachers' Time by Type of School $\ldots \ldots \ldots \ldots \ldots \ldots \ldots \ldots \ldots 40$

Table C 36. Correlation Between Frequency of Use and Perceived Effectiveness of Strategies to Decrease the Teachers' Time by Type of Institution $\ldots \ldots \ldots \ldots \ldots \ldots \ldots \ldots . \ldots 41$

Table C 37. Correlation Between Frequency of Use and Perceived Effectiveness of Strategies to Decrease the Teachers' Time by School Size $\ldots \ldots \ldots \ldots \ldots \ldots \ldots \ldots \ldots \ldots 42$

Table C 38. Schools with a Computer Lab in the School of Nursing . . . . . . . . . . . 433

Table C 39. Institutions with a Computer Lab in the School of Nursing . . . . . . . . . 434

Table C 40. Schools with a Computer Lab in the School of Nursing based on School Size . . 435

Table C 41. Number and Percent of Computers in Four Locations Reported by CAI-user and

Non-user Schools . . . . . . . . . . . . . . . . . . . . . 436

Table C 42. CAI-users and Non-user Schools Reporting Computers in Four Locations . . . . 437

Table C 43. Types of Schools Reporting Computers in Four Locations . . . . . . . . . . . 438

Table C 44. Institutions Reporting Computers in Four Locations . . . . . . . . . . . . . 439 
Table C 45. School Sizes Reporting Computers in Four Locations . . . . . . . . . . . . . . . 440

Table C 46. Percent of Computers in Four Locations Owned by CAI-user and Non-user Schools 441

Table C 47. Number of Computers in Four Locations Owned by CAI-user and Non-user Schools

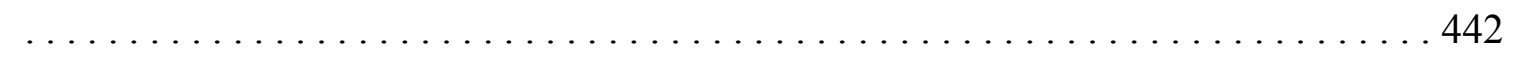

Table C 48. Number of Computers in Four Locations by Type of School . . . . . . . . . . 443

Table C 49. Number of Computers in Four Locations by Type of Institution . . . . . . . . . . 444

Table C 50. Number of Computers in Four Locations by School Size . . . . . . . . . . . 445

Table C 51. Full and Part-time Technical and Instructional Support Personal Employed by

CAI-user and Non-user Schools $\ldots \ldots \ldots \ldots \ldots \ldots \ldots \ldots \ldots \ldots \ldots \ldots \ldots$

Table C 52. Comparison of the Percent of Full and Part-time Technical and Instructional Support Personal Employed by CAI-user and Non-user Schools . . . . . . . . . . . . . . 447

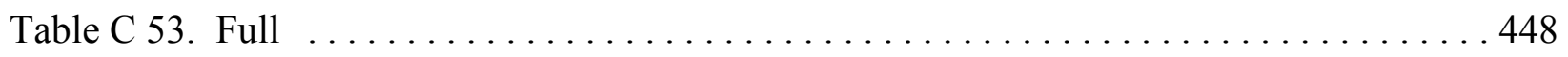

Table C 54. Comparison of the Percent of Full and Part-time Technical and Instructional Support Personal Employed by Three Types of Schools . . . . . . . . . . . . . 449

Table C 55. Full and Part-time Technical and Instructional Support Personal Employed by

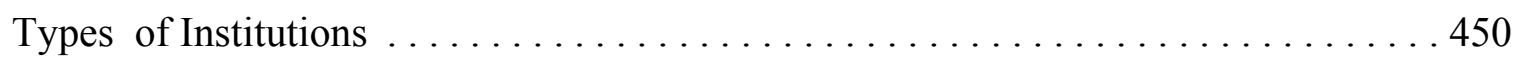

Table C 56. Full and Part-time Technical and Instructional Support Personal by Employed by

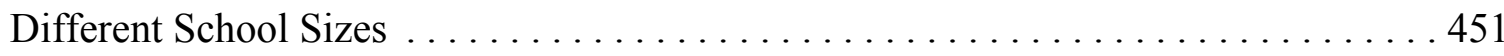

Table C 57. Frequency Distribution and Polygon Graphic Illustration of the Percent of Faculty That Use CAI 452 
Table C 58. Frequency Distribution and Polygon Graphic Illustration of the Percent of Teachers

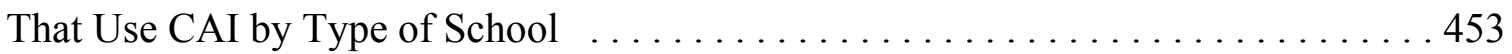

Table C 59. Frequency Distribution and Polygon Graphic Illustration of the Percent of Teachers That Use CAI by Type of Institution $\ldots \ldots \ldots \ldots \ldots \ldots \ldots \ldots \ldots \ldots \ldots \ldots \ldots \ldots \ldots \ldots \ldots \ldots$

Table C 60. Frequency Distribution and Polygon Graphic Illustration of the Percent of Teachers

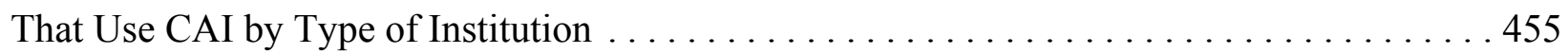

Table C 61. Types of Software Used by Sample Schools by Type of School . . . . . . . . 456

Table C 62 . Types of Software Used by Sample Schools based on Type of Institution . . . . 457

Table C 63. Types of Software Used by Sample Schools based on School Size . . . . . . . 458

Table C 64. Types of Commercially Purchased and Customized Software Used by Sample Schools based on Type of School

Table C 65 . Types of Commercially Purchased and Customized Software Used by Sample Schools based on Type of Institution 460

Table C 66. Types of Commercially Purchased and Customized Software Used by Sample Schools based on Sizes of Schools 461

Table C 67. Ratings of Peers' Effectiveness at Integration Five Types of Software by Type of School: Measures of Central Tendency . . . . . . . . . . . . . . . . . 462

Table C 68. Ratings of Peers' Effectiveness at Integration Commercially Purchased and Inhouse Produced Software: Measures of Central Tendency $\ldots \ldots \ldots \ldots \ldots \ldots \ldots 4$ 


\section{CHAPTER I}

INTRODUCTION

As more nurse educators turn to automated instructional delivery systems such as computer-assisted instruction (CAI) to teach students how to make clinical decisions, the need for information concerning which teaching strategies work best for integrating CAI into the course has grown. The focus of this study is the strategies teachers use and their effectiveness for improving the productivity of the course in terms of student learning, costs, students' time and the teachers' time. The first chapter introduces the study and explains the importance of the research questions.

\section{Background of the Study \\ Clinical Decision-making Is the Backbone of Nursing}

The complexity and unpredictable nature of today's health care environment are placing new demands on nurse educators to prepare students who are equipped with both general nursing knowledge and the ability to make clinical decisions before they graduate (Lowdermilk \& Fishel, 1991). Clinical decision-making was first identified as the terminal objective of nursing education programs in the early 1980's when Benner (1984) began researching the topic. Most of the researchers who have subsequently contributed to Benner's work found that most of a nurse's clinical decision-making skills were developed through practical clinical experience during the five-year period following their formal education (Guyton, 1984; Jenkins, 1985; Jenks, 1993; Maynard, 1996; Tanner, 1989, 1993). Benner's Novice-to-Expert Theory (1984) has been influential in shaping the way nurse educators taught, or failed to teach, clinical decision-making in the interim (Lowdermilk \& Fishel, 1991). More recently, those traditional 
teaching methods have been challenged by a number of innovative learner-centered teaching methods that focus on teaching students to think critically and solve problems, both of which contribute to their ability to make clinical decisions (Lewis, 1997; Maynard, 1996).

\section{Innovative Teaching Methods}

The learner-centered paradigm shift that has taken place in nursing education is evidenced by the teaching methods being used (Adams, Murdock, Valiga \& McGinnis, 2002). The central premise of a new educational philosophy is that proficiency in decision-making is best accomplished by providing students with opportunities to actively practice the higher level thinking skills (Engberg \& White, 1998). For safety as well as financial reasons educators often use simulated rather than real-life clinical experiences for a large portion of students' decisionmaking practice. Some of the learner-centered teaching methods include case studies (with or without live patients) (Bal, Britnell, McCarthy \& Samuels, 1995; Boney \& Baker, 1997; Cioffi \& Markhan, 1997; Lewis, 1997); problem-based learning (PBL) (Engberg \& White, 1998); the use of videotape scenarios (del Bueno, 1983; Gaberson \& Oermann, 1999; Miller, 1990); and computer-assisted instruction (McAlindon, 1992; Perciful \& Nester, 1996; Sittig, Jiang, Manfre, Sinkfeld, Ginn, Smith, Olsen, \& Borden, 1995; Warnock-Matheron, 1997). These methods are being used alone and in combination to teach decision-making skills and to provide students with practice making decisions (Ishida, McKnight, Solem, Tanaka \& Wong, 1994). Increased Use of CAI

Of these teaching methods, the use of CAI has exceeded all of the others for a number of different reasons (Cheek, Gillham, \& Mills, 1998). The first and perhaps most important reason is the developments that have taken place in the area of computer technology. As computers 
were manufactured with greater speed, memory, and power, instructional designers were able to develop more powerful and more sophisticated software programs that could be run from the types of inexpensive desk-top computers schools could afford to purchase. As a result, CAI programs were being designed to incorporate all of the best features of the other student-centered teaching methods but without the high recurrent costs (Barrows, 1983). There are at least four different types of CAI software programs designed to teach clinical decision-making available on the market. Excluding drill-and-practice programs, all of the others, such as simulations, tutorials, virtual reality, and expert systems, are capable of providing students with an almost unlimited, readily available source of active decision-making learning experiences that are difficult to duplicate with any other teaching method.

A second reason the use of CAI has increased is that as computers become more ubiquitous in the home, society, and workplace, consumers' expectations have changed (Goodman \& Blake, 1996). Not only do students expect to use computers for learning but employers also expect their future employees to be computer savvy and prepared as lifelong learners who are able to update their skills and knowledge inexpensively and conveniently at a computer terminal (Sparks, 1989). As the environment around them changes, nursing educators are coming under increased pressure from school administrators, department chairs and peers to use CAI in order to remain current and attract student enrollments (Austin, 1999). Respected organizations, such as the National League of Nursing (NLN), have also encouraged nurse educators to use computer technology in their teaching in order for nursing, as a profession, to keep pace. The authors of the organization's most recent report on the status of nursing education warned that the only way the profession can remain relevant is if nurses individually, 
and collectively, stay on the cutting edge of a technology-intensive health care environment (Adams, Murdock, Valiga, \& McGinnis, 2002).

Nursing Education's Unique Position

A somewhat surprising finding is that when schools of nursing were compared with other schools in higher education, nursing far exceeded all others in the availability and use of computers for instructional purposes (Carty \& Rosenfeld, 1998; Green, 2000). The reason is not that nurse educators are peculiarly attracted to the use of technology but that nursing is a clinically-based profession and as such, must comply with state laws that mandate low teacherto-student ratios in the clinical practice area. As a result, nursing schools must employ more teachers and have higher operating budgets than most other schools in higher education. In response, nursing school administrators and educators continually search for ways to lower their costs in order to keep their productivity figures in line with other schools (Austin, 1999). All of these factors combine to put nursing in a somewhat unique position because any technology, even one as expensive as CAI that can be used to replace costly clinical hours, is apt to save the school money. In addition, when budget cuts do make it necessary to reduce faculty positions, nurse educators turn to CAI and other instructional technologies in an effort to work "smarter" and maintain the teaching loads of the faculty who remain to manageable levels (Austin).

\section{Integration Skills of Teachers}

This explanation helps to explain how it is possible that several studies have also shown that, despite the almost universal use of CAI by nursing schools (Carty \& Rosenfeld, 1998), most nurse educators lack the ability to integrate CAI into their teaching (Austin, 1999; Lewis \& Watson, 1997). In all fairness, the research also shows that similar problems have been 
identified in most areas of higher education. A National Campus Computing Survey conducted in 2000 revealed that institution leaders identified, "assisting teachers in the integration of technology into instruction as the single most important technology challenge facing American colleges and universities over the next two to three years" (Green, 2000, p. 3).

Although it has become widely accepted among educators that the effective use of technology is a critical component of teaching in today's world, most teachers still have limited knowledge about the ways that technology can be used in their professional practices (Thurston, Secaras \& Levin, 1997). One possible explanation for this was proposed by Heinich (1995) who explained that authority for curriculum and instruction decisions in higher education rests with the faculty. However, educators are selected for employment on the basis of their scholarly activity and expertise in their respective professions and not for their knowledge of pedagogy. Many of the same educators who are well qualified to make decisions about the curriculum are not the most qualified persons on campus to make decisions about how instruction relates to student learning (Heinrich). It is also true that educators rarely seek out the advice of media specialists or instructional design experts on such matters. Instead, most of their decisions are based on information from the literature, discussions with other educators, observations of their own prior experiences and, rarely, from evidence-based research (Angel, Duffey \& Belyea, 2000).

Another plausible explanation for the difficulty that educators have integrating CAI into the courses they teach is the complexity of the task. In one report entitled "Technology and Teachers: Making the Connection" (Office of Technology Assessment, 1995, p. 161) the report writers noted, "Contrary to popular opinion, learning how to use technology and mastering 
software is simply not enough; teachers also need to learn how to teach with technology and how to harness the tools for instructional ends which is a much more complex issue." Hammond, Gardner, Heath, Kirby, Mayes, McAleese, Mullings, and Trapp (1992) surveyed a large group of seminar participants from the U.K. to determine why, despite coordinated efforts to promote the use of CAI in university teaching, it was so seldom used. Answers by survey respondents revealed that faculty had many personal reasons for not using technology such as fear, laziness, loss of control, and the failure of promotion and tenure policies to reward their efforts to do so. Of those who wanted to use CAI, most indicated the primary reason they did not was because they lacked the time for such things as modifying the course, evaluating the available software or developing the materials themselves. Hammond and his colleagues concluded that it is unrealistic to expect an entire educational system to go from a world almost totally without technology to one where most learning occurs from a computer. It is too big a jump and too few people recognize how much time and work is involved and how much educators need to learn to do it (Hammond et al.).

\section{Complexity of the Task Underestimated}

Bates (1999) explained that teachers need to concentrate on designing the learning experience and not on teaching the same things in the same ways with the addition of technology. A fundamental question that needs to be addressed is not, "should" technology be used to replicate traditional instructional methods but "how should" technology be used to change teaching and improve the quality of teaching and learning (Bates, 1999). Thede (1999) expressed the same idea, a little differently, when she proposed that the key to successful CAI application is the integration of the technology rather than in merely adding it onto a previously 
designed course. A predominant view in North American universities and colleges is that the traditional form of group, or face-to-face instruction is still the preferred and most effective form of education, and that the closer technology-delivered instruction can mimic this ideal, the more effective it will be. However, in Bates' opinion, any CAI program that can only mimic traditional instruction is not worth the investment. To be cost effective, CAI needs to be able to go far beyond what is being done in a traditional classroom.

\section{Integration Strategies}

As previously mentioned, in order to use CAI software for instructional purposes, educators must first recognize the need to integrate the CAI learning experience with the rest of the course and then to learn how to do it (Thede, 1999). Fortunately, there are a number of wellknown theories by such notables as Briggs (1991), Dick and Carey (1991), Gagne' (1985) and Merrill (1983) who, on the basis of decades of research, described the principles of instructional design and how any instructional technology should be integrated into a course. One highly regarded reference on the topic is a text by Kemp, Morrison and Ross (2001) that was used to identify the integration strategies that were included in the data collection instrument of this study.

\section{Integration Strategies Defined}

Integration strategies are defined as the skillful planning and management of instruction by the teacher in ways that increase the overall effectiveness of the course. The four basic reasons teachers use CAI include: (1) to increase student learning, (2) decrease the time students spend on the learning task, (3) decrease course costs, or (4) decrease the time the teacher spends. An integration strategy, such as include the scores earned on tests "embedded" in the CAI as part 
of the course grade may be used by one teacher to motivate students to learn the content, by another to decrease the time students spend on the task by having them complete the test as they work their way through the CAI assignment, and by another to reduce the time the teacher spends preparing for and administering a test on that content. Some integration strategies are only used by teachers for one purpose, and therefore are only included under one heading, while others may be used for all four purposes and are included under all four headings on the "Integration of CAI Questionnaire."

\section{Educational Productivity}

Educational productivity is a method of measuring effectiveness by looking at the relationship between the resources expended to provide instruction and the outcomes of the instruction (Levin, 1983). It has become an increasingly important concept in higher education in recent years as schools search for ways to improve educational effectiveness while at the same time adjusting to tightened budget constraints. There are three broad categories of costs that are commonly included when calculating CAI costs that include: (1) the cost of the technology (i.e., hardware, software and peripherals), (2) personnel costs (i.e., teachers, assistants and support personnel) and (3) the costs for the equipment and upkeep of the facilities (Levin). The most common ways teachers use teaching strategies to improve the productivity of a course are to either intervene to increase student learning in measurable ways or use the intervention to hold the level of learning constant while either increasing course revenues or decreasing course costs. The usual method of increasing course revenues is by increasing the number of students enrolled in the course (Massy \& Wilger, 1998) and since the teachers' salaries are almost always the greatest costs of the course, the usual method of reducing course costs is to reduce the time the 
teacher spends teaching or working at teaching-related tasks (i.e., test construction, administration, grading and record keeping) or to reduce the teacher's time indirectly by significantly reducing the amount of time it takes for students to learn (Dean, 1989).

Integration strategies may be classified as preinstruction, midinstruction or postinstruction activities or decisions the teacher makes to integrate the CAI learning experience so that it becomes part of the total educational plan (Bates, 1995a). A brief discussion of how the integration strategies that were selected for inclusion in this study were chosen and how they relate to measures of productivity will follow.

\section{Strategies to Increase Learning}

Some of the preinstructing integration strategies that are primarily intended to improve student learning are decisions or actions the teacher typically makes before the course begins such as evaluating and selecting commercially-produced software or developing customized software that closely matches the objectives, skills, and content of the course (Conklin, 1983; Goyne, Mc Donough, \& Padgett, 2000; Griepp, 1988; Kemp et al., 2001) to the needs and abilities of the students (Gleydura, Michelman \& Wilson, 1995; Khoiny, 1995). Other preinstruction strategies that are intended to prepare students for the instruction include activities such as informing students of the objectives of the CAI program, motivating by explaining why the content is important and how the content or skills taught in the CAI relate to the rest of the course (Kemp et al., 2001). Still others include providing or arranging for someone else to demonstrate how to use the computer and each new software program before students do the assignment (Thede, Taft \& Coeling, 1994).

Two examples of midinstruction strategies that a teacher may decide to use to improve 
student learning are to include the scores students earn on "embedded" CAI tests as part of the course grade or include a sufficient number of test questions from the content of the CAI as part of an "in-class" examination. The rationale for both strategies is to promote the perception in the minds of the students that the CAI assignment is an integral and important part of the course, and because it is, the time spent on the assignment will significantly impact the course grade (Kemp et al., 2001; Thede et al., 1994).

A teacher-directed in-class or post clinical "discussion" to help students relate the content of the CAI to the rest of the course is an example of a post-instruction strategy that is intended to increase student learning. Assigning a written critique, a worksheet, or some other "follow-up" assignment in order to reinforce and evaluate student learning as well as provide the teacher with an opportunity to correct students' misconceptions are others (Kemp et al., 2001; Laurillard, 1993). Most of the postinstruction strategies involve some form of "evaluation" to ensure the students who use the CAI program accomplish the course objectives or to collect student feedback about the CAI learning experience so that appropriate adjustments can be made to the course (Dick and Carey, 1991). Another is to have an "alternative," remedial assignment ready for students who fail to accomplish the course objectives using the CAI program (Kemp et al., 2001).

Strategies to Decrease the Students' Time

Because human learning requires information processing, which consumes massive amounts of energy, the role of the teacher in "integrating" CAI is to follow good instructional design principles so that learners can conserve time and energy and direct their attention to where it needs to be, on the learning task (Gibbons \& Fairweather, 1998). The research findings 
indicate that students learn as much in less time using CAI as they do with lecture and clinical practice (Fletcher, 1992; Weis \& Guyton-Simmons, 1998). One dated but dramatic finding showed that the CAI group learned as much as the lecture half of the class but cut their learning time from 66.5 to 23.5 hours (Bitzer \& Bordreaux, 1969). Even more important to this study, the research also shows that not only does CAI decrease learning time but there are a number of anecdotal accounts in the literature that support the idea that teachers can impact the time students spend on a CAI program using many of the same integration strategies that are intended to increase learning. Some of the best illustrations are studies where teachers failed to use the integration strategies. One example came from the Thede et al. (1994) study where students reported the reason they wasted considerable learning time was due to a failure on the part of the teachers to prepare them beforehand or to ensure that there were "technical" and "instructional" support personnel available to help novice students who needed it.

\section{Strategies to Decrease Costs}

Clark and Sugrue (1988) noted that the research does not support the notion that the psychological aspects of learning are related to any media. Students who set about achieving the same learning tasks and who had equal preparation and motivation to learn did equally well regardless of the media. However, there is considerable and dramatic evidence that media does influence the economic elements of learning. That is, under certain conditions, media can dramatically influence the cost of learning. In a review of cost-effectiveness studies of media (primarily devoted to computer-based instruction) Henry Levin (1986) concluded the costeffectiveness of CAI was relatively poor in most of the better evaluation studies. However, Levin also found that there were dramatic cost-effectiveness differences for the same CAI 
program implemented at different sites. When sites made a determined effort to promote full utilization of the media and software, the cost-effectiveness ratio increased by a factor of 50 percent. In some cases, when the same CAI program was implemented at different schools by different teachers, the cost-effectiveness ratio changed significantly by as much as 400 percent. The findings strongly suggested different strategies for managing the media and the way the media was implemented could greatly influence the cost of achievement from computers and probably other media as well. Since one would expect media courseware that was administered by a computer would produce the same level of achievement at different sites, Levin concluded it was most likely management and organizational issues that influenced costs and, in some cases, inhibited achievement (Levin, 1986).

There are three integration strategies teachers use to assign a CAI program that are commonly referred to in the literature as "modes" of learning, or instruction. Depending on a number of variables, the teacher may decide to integrate the CAI by assigning students to work as "individuals" or in "small groups" or the teacher may use the software in the classroom with a computer-projection system for a "whole-class" learning activity (Thede, 1999). Each mode of learning has been shown to offer a complex mix of advantages and disadvantages that could impact the cost of the course.

The research shows that when teachers integrate CAI as a supplement to the regular mode of instruction for remedial or enrichment purposes instead of using it to "replace" the traditional teaching method of teaching, the productivity of the course falls (Bates, 1999). When teachers use both CAI and teacher-delivered instruction to teach the same content, not only is the unit costs of the instruction duplicated but the course productivity is lowered even further 
because the teachers' and the student's time are nearly doubled (Dean, 1989). Another common mistake teachers make is to designate a CAI assignment as "optional" rather than a "required" part of the course (Bates). Motivated by a desire to be fair to off-campus students, the teachers designate technology-delivered assignments optional and then, again, motived by a desire to be fair, the teacher seldom includes the "optional" information covered solely by the technologydelivered instruction on the course tests. Once the students become aware that they can pass the tests without completing the assignment, they stop doing the CAI assignment and the end result is that an expensive, potentially valuable learning resource ends up being a costly liability rather than an asset to the productivity of the course (Greipp, 1988; Collis \& Moonen, 2001).

Ensuring that there are "technical" and "instructional" support personnel available to assist students who need it, either by phone or on-site at a computer laboratory, has been shown to positively impact student learning and decrease the students' time, both of which are important productivity measures (Thede et al., 1994). However, the ongoing costs for support personnel salaries are so expensive that teachers who elect to include the costs as part of the course budget are likely to report a significant increase in the course costs (Dean, 1989; Levin, 1983).

Strategies to Decrease the Teachers' Time

There are several integration strategies that are implemented specifically to reduce the teachers' time. The first, and probably most obvious way, is to use the CAI to "replace" the usual classroom or clinical instruction (Bates, 1999). One of the most widely recognized advantages of CAI is that it can be assigned for "individualized" self-paced learning by students working alone or in "small groups" outside the classroom. A second teacher time-saving 
strategy is to use CAI software for a "whole-class" presentation as a part or to entirely replace the conventional mode of instruction. Thede (1999) and Goodman and Blake (1996) are a few of many authors to report CAI simulations, virtual reality and tutorial programs are being used by teachers to visually illustrate difficult principles and concepts so that the amount of time it normally takes for a lecture-discussion class is reduced. A third strategy is to select or design software that includes "embedded" tests and automated methods of evaluating and reporting students' grades or use test questions from embedded tests as part of an "in-class test." The rationale is to eliminate, as much as possible, the time the teacher spends on tests without compromising student learning (White, 1995). Again, by ensuring there are adequate "technical" and "instructional" support personnel available to assist students, the teacher can save considerable amounts of time simply by eliminating the need to provide the services themselves, which could have a significantly negative impact on the productivity of the course and their teaching effectiveness (MacKnight, 1995).

In conclusion, nurse educators are under pressure to find ways to teach students clinical decision-making skills prior to graduation by a number of dynamic changes that are taking place in the health care and educational systems. Nursing schools have adopted many different learner-centered teaching strategies to teach and provide students with practice making clinical decisions. Developments in computer technologies, as well as social, professional, and economic factors are all contributing to the development of a trend toward the increased use of CAI in nursing education. The literature indicates nurse educators are using CAI more often than many of their contemporaries in higher education but that their knowledge of how to integrate it is poor. A comprehensive review of the nursing education literature revealed that 
few studies have considered the use of CAI from a pedagogical perspective and that only one qualitative study by Thede et al. (1994) addressed the way nurse educators integrate CAI as a primary topic of interest. The literature also indicates the way teachers integrate CAI into the course makes a large difference in both the cost-effectiveness and productivity of the course. For these reasons, this is a topic of considerable importance to nurse educators and it may also contribute to the larger body of knowledge concerning educational practices.

\section{Purpose of the Study}

The purposes of this study were to identify the integration strategies nurse educators use most frequently to integrate CAI designed to teach clinical decision-making into the courses they taught and those that were perceived to be effective for increasing student learning, decreasing the students' time, course costs and the teachers' time. The study also identified the characteristics of schools and teachers that nurse educators perceived to integrate CAI most effectively. Another purpose was to identify the types of software nursing schools throughout the United States used to teach clinical decision-making as well as the numbers of commercially and in-house produced software programs that were used and which types of software nurse educators perceived teachers at their schools were able to integrate effectively.

\section{Statement of the Problem}

The problem statement for this study was nurse educators did not know which integration strategies were being used or which ones were perceived of as effective for increasing student learning, and decreasing costs, the students' time and the teachers' time. Nurse educators also needed to know the characteristics of schools and teachers that were perceived by participants as integrating CAI effectively, which types of CAI software were being used to teach clinical 
decision-making, what types of in-house or commercially produced programs were being used and which types of software participants perceived their peers were able to integrate effectively into their courses.

\section{Study Questions}

1. What integration strategies have nurse educators used to integrated CAI programs designed to teach clinical decision-making into the courses they taught?

2. How effective did participants perceive the integration strategies were in terms of increasing student learning, decreasing course costs, the students' learning time and the teachers' time?

3. What are the key features of the schools and educators that were perceived by participants to integrate CAI designed to teach clinical decision-making effectively?

4. What types of CAI software did nurse educators use to teach clinical decisionmaking?

5. How effective did participants perceive teachers at their school were at integrating the different types of CAI software (simulations, tutorials, etc.) and commercially purchased and inhouse produced software into the courses they taught? 


\section{CHAPTER II}

\section{REVIEW OF THE LITERATURE}

There is a plethora of literature on the topic of CAI as an educational intervention. Some of the publications are empirical studies but most are descriptions of new and innovative ways CAI software applications are being used in higher education to teach a broad range of topics and skills. Many of the descriptions focus on the unique features of the software applications and for the sake of clarity and brevity the authors describe the programs as if they were standalone units of instruction. In fact, in almost every instance CAI programs are small units of instruction that the teacher merges with a variety of other instructional units to form a cohesive course of instruction. A few of the authors discuss how the CAI is integrated into the course, but for the most part, the literature is silent on the topic of "integration" (Pierson, 2001; Thede et al., 1994). There is also little in the literature about the role of the teacher in selecting and implementing integration strategies that increase the effectiveness of the CAI and, ultimately, the course. This scarcity of information is both troublesome and surprising in light of a large number of studies and reports from experts in the field who have noted that most educators view the "integration of computer instruction" as a major professional concern and that they have expressed a need to know more about the topic (Austin, 1999; Bates, 1999; Bolwell, 1998; Green, 1996, 2000; Hammond et al., 1992).

In an effort to logically link this study with what is already known about the topic, the literature review will begin by exploring the first study question which seeks to identify how often nurse educators use the integration strategies under investigation in this study. The discussion will begin with a brief overview of how often CAI is used by nurse educators and 
what is known about how they integrate CAI into their courses. The second study question addresses the effectiveness of the integration strategies that are being used. The review will look at the effectiveness of CAI in terms of learning outcomes and then, what the literature says about the effectiveness of CAI for teaching clinical decision-making. Finally, the focus will narrow to a discussion of the primary topic of the study, the integration strategies and what the literature, in terms of instructional design theories and experts in the field have said about how they may, or may not, be used to increase the effectiveness of a course in terms of the productivity measures under consideration here. The review ends by looking at the literature as it relates to the other study questions such as the types of software that are being used to teach clinical decisionmaking and the characteristics of the schools and educators who integrate it effectively.

The Use of CAI in Nursing Education

History of the Use of CAI

For more than thirty years CAI has been recognized as an effective tool for improving learner performance and achievement in nursing education (Cohen \& Dacanay, 1994). Mikan and Aiken's (1992) nationwide survey included 513 undergraduate nursing schools throughout the United States and the findings revealed that computers were used in both pre-nursing (55\%) and nursing courses (77\%). Computer-assisted instruction was primarily used as an enrichment supplement to classroom and clinical teaching, rather than to replace traditional instruction. The types of software programs being used at that time were simulations, tutorials, drill and practice, and testing programs. Nursing-related content areas were calculations, adult nursing, clinical case studies, clinical decision-making, and maternity nursing (Mikan \& Aiken). In another study, Bass (1991) surveyed 211 nurse educators from Georgia to determine their frequency of 
use of CAI and found that only 34.6\% used drill and practice, tutorials, games, problem-solving simulations and/or interactive video in at least one course during the period from 1988 through 1990. Oermann (1997) surveyed educators from 122 associate programs that were randomly selected from four different geographic regions of the United States and found $89 \%$ of the schools required the use of computers for instruction in their nursing curricula.

\section{Measures of Educators' Skills}

When program directors were asked to rate the computer competency of their faculty and students on a scale of 1 (not important) to 4 (most important), the directors rated the competency levels of faculty members at "using PCs" as a 3.84 which they perceived as more important than any of the other options. The same directors rated "being able to use CAI programs" at 3.78 for students indicating it was the most important of the four options (i.e., using a PC, using software to document patient care, entering patient data, or retrieving data). There was no statistically significant difference among the four geographic regions of the country.

The most recent study on the topic conducted by Carty and Rosenfeld (1998) looked at the status of computer technology in nursing education in the United States. The survey included 190 randomly selected NLN accredited schools. The results showed that CAI was used almost universally by all of the respondents. A large majority (70.9\%) of the schools that used CAI required its use by undergraduates as part of the curriculum. More than half (61\%) used interactive video disc instruction (IVDI) and $70.7 \%$ of those required its use by students in the undergraduate curriculum.

In 1989 Sparks reported the nursing teachers at that time were seriously limited in their ability to use computers for educational applications. Ten years later, Austin (1999) did a 
broader follow-up survey to determine faculty competency levels at performing 60 computer literacy skills and how respondents rated their ability to integrate the skills into the curriculum. The study was a cross-section of 184 nurse educators from 18 publically-funded, NLNaccredited BSN schools in the New England region. The results indicated that over $50 \%$ of the educators reported performing 21 of 60 computer skills "well” or "very well," but only 50\% reported that they were only integrating three of the 60 computer skills in their teaching practice either "moderately" or "extensively." The participants scored a mean of 138.39 points out of a maximum 240.00 possible points. Seventy percent achieved a total score of 147 or less on skills that were rated as "highly important" by a national study of nurse educators (Bryson, 1991). One particularly interesting finding was that $53 \%$ of the teachers reported that they had "little" or "no" ability to run nursing software as a learning tool or for evaluation purposes; $46 \%$ had "little" or "no" ability to discuss CAI and its use as a teaching-learning tool and that $80 \%$ had "little" or "no" ability to use a multimedia presentation package or to prepare a teaching presentation. As would be expected, teachers who could comfortably perform computer skills were found to be more likely to integrate them into the curriculum through their teaching (Austin).

Austin (1999) identified the need to provide ongoing, systematic continuing education programs for nursing teachers both in the basic use of computers and for the use of computers for nursing professional practice. However, she also noted that the success of the offerings would be limited since $56 \%$ of the respondents indicated they did not usually attend continuing educational unit (CEUs) courses when they were offered. Austin concluded that the participants' scores indicated there were extensive areas of self-reported weaknesses in the integration of 
nursing computer skills into teaching practice in all domains. Of the 60 skills listed, only three were being integrated into teaching practice either "moderately" or "extensively" and only one skill (i.e., discuss CAI and its use as a teaching-learning tool) could be performed "well" or "very well" by $50 \%$ or more of the participants (Austin).

Several investigations have shown that educators identified a lack of adequate preparation as a significant barrier to the adoption of technology innovations (Dupagne \& Krendi, 1992; Handler, 1993). On the other hand, educators who feel adequately prepared to use computers in the classroom are more likely to apply the technology and initiate computer use in their educational programs (Handler, 1993; Podemski, 1981).

Interventions to Improve Educators' Skills

Lewis and Watson (1997) used a standardized questionnaire (The Stages of Concern) to measure nursing faculty members' concerns about the adoption of computer technology and an author-developed survey to determine the level of technology use before and after a series of faculty development computer workshops. Initially, most of the 28 participants were low-level computer users. After a series of five computer workshops, many of the teachers showed statistically significant $(\mathrm{P}<0.05)$ improvements in their comfort levels in three of the technology areas one of which was the use of software. Of the ten individuals who responded to questionnaires about barriers to the use of computers, time to learn $(n=8)$ and insufficient numbers of computers in their offices were identified as the two greatest barriers $(n=7)$. Some of the other barriers included the following: too many responsibilities $(n=3)$, lack of software $(n=2)$, and a lack of availability of technology equipped classrooms $(n=2)$. Overall, workshop participants experienced increased comfort levels and interest in learning about and using 
computer applications (Lewis and Watson).

In summary, the literature indicates that there has been a trend toward the increased use of CAI over the past 30 years in nursing education. By the early 1990s, educators were primarily using it as an enrichment strategy but not to replace traditional instruction (Miken \& Aiken, 1992). Several large studies found that CAI was almost universally used by nursing schools across the United States and that most schools required students to use it as part of the curriculum (Carty \& Rosenfield, 1989; Oermann, 1997). Austin (1999) found that nurse educators reported they lack both the knowledge and skills to integrate CAI into the curriculum. Out of the 60 skills that pertained to integrating computers into their teaching practice, only three were being used moderately or extensively and only one could be performed by $50 \%$ of the participants. Over $80 \%$ had little or no ability to use a multimedia presentation package or prepare a teaching presentation. There were extensive areas of self-reported weakness in integrating nursing computer skills in all domains of nursing education (Austin). The lack of adequate preparation has been identified as a major barrier to the use of CAI by educators. Lewis and Watson (1997) found that a series of five computer workshops was enough to significantly reduce faculty members' concerns about using computer technology for teaching and heighten their levels of interest in its use.

The focus of the literature review will shift to the effectiveness of CAI as an educational intervention and its impact on the measures of productivity that are of interest in this study. Since the primary topic of this study is the "integration strategies" teachers use and not CAI, the literature review on the topic of CAI will be limited to a brief overview of the highlights of the research and not an in depth review. 
Use of CAI in General Education

A national survey from the Higher Education Research Institute at UCLA reported 87\% of the faculty believed students' use of computers enhanced learning and that the intelligent use of CAI technology could improve the quality of teaching (Sax, Astin, Korn \& Gilmartin, 1999). The findings were somewhat surprising in light of the fact that most of the early research that was designed to measure the effectiveness of CAI was called into question in the early 1980s and more recently, researchers generally view studies that compare the relative effectiveness of computer-based teaching with traditional face-to-face teaching as a futile effort since the results of such comparisons (i.e., no significant difference) are already known (Epper, 2001; Russell, 1999).

\section{CAI vs. Traditional Teaching Methods}

At the very least, studies from a number of disciplines have shown that students learn as well with CAI as with so-called, "traditional methods" for a number of reasons. Niemiec and Walberg (1987) listed sixteen major reviews beginning with studies from the 1960s that assessed the relationship between CAI and achievement. The list included eleven meta-analysis studies and five other reviews that used quantitative percentage comparisons. The findings across all educational levels showed that CAI was more effective than traditional instruction with an average effect size of (.42). Niemiec and Walberg concluded the CAI groups had a "substantial, but not overwhelming advantage" over the traditional instruction groups. The highest student gains were at the elementary school level (.46), the lowest were at the college/university level (.26), and high school students were in the midrange (.32) (Niemiec \& Walberg).

In another early meta-analysis study, Kulik, Kulik, and Cohen (1980) looked at the 
effectiveness of CAI for college-level students and found small gains in measures of achievement for CAI over traditional teaching methods. In another meta-analysis, Kulik and Kulik (1991) reviewed studies of higher education that spanned the twenty year time period from 1966 to 1986 and found similar findings with a mean effect size of .30. Most studies also reported more favorable attitudes and more positive ratings of the quality of instruction and the subject matter in the CAI groups. Bunderson, Baillio, Olsen, Lipson, and Fisher (1984) compared learning and students' attitudes with CAI and traditional lecture in an introductory, college-level Biology course and found the gain scores of the CAI group were significantly higher than the lecture group on the pre-post-test comparisons and pre-retention test comparisons.

\section{It's Not The Media}

In 1983, Clark began a widely publicized debate on the topic when he published an article on media research and learning. In a follow-up series of articles, Clark argued that no media has a distinct advantage over any other and that it was the uncontrolled effects of the instructional method and content, as well as novelty, which account for any learning benefits that were attributed to CAI (Clark, 1985a; 1985b). Clark (1983) proposed that "media" and the "method" are often confused and as a result, researchers often made misleading statements regarding the attribute of effectiveness. Clark interpreted the findings by Kulik and his colleagues (Kulik, Bangert \& Williams, 1983; Kulik \& Kulik, 1987; Kulik \& Kulik, 1991) that showed consistently higher effect sizes for teacher, duration of study, and publication as supporting his view; when the same teacher was used and the duration of the study is more than short-term, the uncontrolled effects of method and novelty are reduced and consequently the 
effect size dropped substantially. Clark (1983) also believed that publishers' editorial bias in selecting studies that show significant effects resulted in those studies being published while others that did not report significant effects were not. He also proposed the time savings that were noted when CAI was used actually reflected better designed instruction. In rebuttal, Kulik and Kulik (1991) acknowledged the study feature effects cited by Clark were troublesome, but noted other alternative explanations were just as viable; the converse of editorial gate-keeping might also be true and studies of lower quality may not have been accepted for publication; shorter studies may have been better controlled, and in studies with the use a one teacher control, diffusion of the methods used in CAI may have raised the standard of teaching in the control group as well. In all of these cases, effect sizes would be underestimated rather than overestimated. They concluded the reasons for the differences were imperfectly understood and agreed with Clark that more research was needed (Kulik \& Kulik).

Fletcher-Flinn and Gravatt (1995) also used a meta-analysis to examine the effectiveness of CAI across a broad range of variables that were chosen specifically to answer the questions raised by the Clark vs. Kulik et al.debate. The initial findings were consistent with past reviews in showing the same CAI advantage over traditional instruction. The average effect size of (.24) had the effect of raising the post-test scores in a typical study from the $50^{\text {th }}$ to the $60^{\text {th }}$ percentile. The findings were similar to the Kulik and Kulik study (1991) which reported an effect size of (.24) for studies from 1966 to 1974; (.36) for studies from 1974 to 1984; and (.30) for studies from 1974 to 1985 . However, Fletcher-Flinn and Gravatt also found the learning gains were, as Clark had suggested, often an artifact of poor research design and not due to the media. In studies that controlled for both teacher and materials, studies that were longer in duration and 
those using the pencil and paper equivalents of CAI demonstrated no learning advantage over the CAI group. Fletcher-Flinn and Gravatt concluded just as Kulik and Kulik (1991) and Clark (1983) had that CAI was generally of higher quality because a lot of time and effort were put into the design, the instructional objectives tended to be clearer, the instruction was sequenced with feedback, and materials encouraged active participation instead of passive inactivity on the part of the learner. So, while the research clearly showed CAI does not seem to result in any learning benefit that can be attributed to the computer as a medium, Fletcher-Flinn and Gravatt went on the conclude that learners still gained more from the typical CAI learning experience than from most other classroom learning experiences. In agreement with Clark's findings, Kulik and Kulik found in a later study that most researchers agreed that students had more favorable attitudes toward CAI instruction and rated the quality of instruction and the subject matter of CAI more favorably which, they concluded, probably contributed to higher learning achievement scores (Fletcher-Flinn \& Gravatt).

In another meta-analysis of over 200 studies to determine the effectiveness of CAI on various productivity measures, Roblyer (1988) found that computer applications had a statistically significant positive effect $(p .<0.05)$ in a majority of the areas examined. While significant results were found at all grade levels, there were larger effects at the college and adult levels than at elementary and secondary levels. Statistical analysis permitted no firm conclusions about the superiority of any type of computer application (i.e., drill and practice, tutorials, simulations, problem solving) but most types of skills were found to profit from computer applications (Roblyer).

In summary, many faculty believe that when CAI is used intelligently, it not only 
enhances learning but it can also improve the quality of teaching (Sax, Astin, Korn \& Gilmartin, 1999).

Many researchers have compared CAI with traditional teaching methods by investigating different measures of student learning and have found that the CAI group had a substantial, but not overwhelming advantage over the traditional group at all educational levels. Kulik and his colleagues (1983) used meta-analysis techniques to evaluate the results of earlier studies at the college level and found in favor of CAI for achievement, reductions in students' learning time and more favorable attitudes toward the CAI instruction. In response, Clark (1983) wrote a series of scholarly articles in which he argued that the CAI media was merely a vehicle and as such, made no difference in student learning but that the more favorable results were actually due to research design flaws as well as editorial bias in selecting statistically significant studies for publication. Instead of an effect of media, Clark argued that the improved performance and time-savings were due to the fact that instruction was designed better and that when the same teacher designed both, the differences vanished. In a follow-up study, Fletcher-Flinn and Gravatt (1995) tested the same variables Clark questioned and found that while the Kulik and Kulik (1991) calculations were correct, most of the learning gains were an artifact of poor research design and not due to the media. They also concluded that learners gained more from the typical CAI learning experience because the instruction was designed better and students enjoyed active learning more than passive forms of instruction (Fletcher-Flinn \& Gravatt). More recently, almost no researchers test for differences in learning between CAI and traditional instruction because the results of "no significant differences" are already known (Epper, 2001; Russell, 1999). 


\section{Effectiveness of CAI for Nursing Education}

Computers have been used in nursing education since the late 1960s to teach topics such as respiratory assessments (Bratt \& Vockell, 1986), nursing research (Gaston, 1988), nursing management of hypertension (Huckabay, Anderson, Holm, Lee, 1979), and surgical nursing (Conklin, 1983).

\section{Early Studies}

Many studies in the field of nursing education found no significant differences in achievement between the experimental and control groups (Abrams \& Streit, 1986; Levenson, Morrow, \& Signer, 1985-1986; Hamby, 1986; Schare, Dunn, Clark, Soled, \& Gilman, 1991). A number of early studies used a large mainframe computer system called PLATO and different locally-developed software programs to compare CAI with traditional teaching methods. Bitzer and Bourdreaux (1969) also used PLATO to teach maternity nursing and found that while both groups met the objectives, the differences between them were not significant.

Huckabay et al. (1979) used a teacher-developed CAI program as a supplement to regular classroom instruction to teach 31 nurse practitioner students the care of a hypertensive patient. The findings revealed that the CAI group were able to transfer what they learned from the CAI to the practice area better than those taught by lecture/discussion alone (Huckabay et al.). Boettcher, Alderson and Succucci (1981) used a locally developed CAI software program to teach 83 BSN students psychopharmacology and found the CAI training was as effective as printed instruction for both recall and the transfer of knowledge to the clinical area (Boettcher et al.). Conklin (1983) taught 34 third year BSN students surgical nursing using a supplemental CAI package and found the CAI group scored significantly higher $(\mathrm{p}<0.001)$ than those who 
used textbooks as supplements. Benton (1989) used CAI to replace conventional teaching for 55 in-service nurses and found the CAI group scored higher, transferred what they learned to the clinical area better and accomplished the learning objectives in a shorter amount of time.

\section{Quality of the Research Questioned}

Lewis, Davis, Jenkins and Tait (2001) reviewed the international nursing and health-care databases from 1966 to 2001 to evaluate the research and to see if any meaningful conclusions could be drawn about the usefulness of CAI. An electronic search resulted in hundreds of titles that were quickly narrowed down to only 25 empirical studies that met the study criteria. Of those, many had serious design flaws such as too small sample sizes, the lack of a control group, no attempt to control for incidental learning or maturation effects, etc. There were two metaanalysis studies conducted by Belfry and Winne (1988) and Cohen and Dacanay (1994) that yielded questionable results because there were inconsistent findings in the calculations of the original data. Only the studies conducted by Huckabay et al. (1979), Conkin (1983) and Benton (1989) (mentioned above) were comprehensive and designed well enough to be given credence (Lewis et al.).

In addition to study design flaws, Lewis et al. (2001) noted that three other factors make it almost impossible to derive any substantive evidence from a review of the nursing studies concerning the utility of CAI as an "effective" teaching tool. The first, intra-study variability made it almost impossible to minimize the effect of non-CAI variables. Bias was often introduced by the variable parameters of the student population (i.e., differences in age, previous knowledge, preferred learning style, etc.) and due to the parameters associated with the control of the delivery of the CAI within an educational setting. The second, inter-study variability 
occurred as a result of the highly specific nature of most of the CAI studies (i.e., specific design objectives and learning outcomes, type and style of CAI program, etc.) which made it difficult to extrapolate from the results of individual studies to general situations. In most cases, a particular CAI package that was effective in one situation could not be assumed to be effective in another. The third variable, the technological developments that occurred over the 30 year time period meant study results from the 1980 s were, for all practical purposes, meaningless in the 2000 s. The rapid pace of changes that occurred in the levels of sophistication and expectations of endusers meant almost every year there were new expectations and new criteria for what was meant by good instructional materials. As a result, a quantitative body of evidence that is the sum of multiple studies is simply not possible. Lewis et al. concluded, the results of individual studies should only be interpreted meaningfully within the context of that study.

A summary of the literature concerning the effectiveness of CAI in nursing education revealed that the use, sophistication, ease of access, and types of CAI programs being used for nursing education have grown considerably over the years. Just as in general education, many of the early studies found no significant differences in student achievement regardless of the topic being taught or the student population tested. A few researchers found students were better able to transfer learning to the clinical area with CAI (Conklin, 1983: Huckabay et al., 1979; Boettcher et al., 1981). With a few exceptions (Benton, 1989; Huckabay et al.), most studies looked at CAI that was used as a supplement to traditional instruction. Many authors discussed the reason they used it only as a supplement was because the research on its effectiveness as a stand alone intervention was still inconclusive (Greipp, 1988; Mallow \& Gilje, 1999). A more recent review of the nursing literature from 1966 until 2001 conducted by Lewis et al. (2001) 
revealed there were thousands of articles on the topic of CAI but only 25 were empirical studies and of those, all but 3 had serious design flaws and problems with the methodology. Lewis et al. (2001) determined that a substantiative conclusion about the impact of CAI from the available literature was not possible. Most of the studies were potentially biased due to the variability of subjects and lack of the researchers' ability to control the delivery of treatments in a school setting. Inter-study variability made generalizations from one study to another almost impossible which meant a CAI package that was shown to be effective in one setting may not be effective in the next (Lewis et al.).

Next, the review will discuss the literature concerning the use of CAI to both teach and provide students with practice in clinical decision-making.

\section{Uses of CAI to Teach Clinical Decision-making}

The research shows that novice students often make rudimentary decisions based on life experiences and not what they learn in the traditional lecture style classroom (Thiele, Baldwin, Hyde, Sloan \& Standquist, 1986). As a result, most of their decisions are inappropriate, superficial, and lack established priorities. In a traditional educational setting, students are taught a variety of nursing actions such as observation, interview techniques, physical, and mental assessment procedures and a vast number of psychomotor skills and then, armed with these skills and some degree of basic nursing knowledge, they are expected to somehow make appropriate clinical decisions. Thiele et al. found that just as psychomotor skills must be practiced over and over, students also need to be taught how to make clinical decisions and then, provided with opportunities to practice the skills. 


\section{A Substitute for Clinical Practice}

Since nursing is a practice-based discipline, what students are able to do in the clinical area is more important than how well they perform in the classroom but financial and practical constraints make it necessary to teach the bulk of nursing theory in a classroom or school-based clinical laboratory setting (Gaberson \& Oermann, 1999). In general, most educators agree that there are many technical, interpersonal, and organizational type skills associated with clinical decision-making that students can only learn in the clinical setting. Cheek et al. (1998) noted there are discipline-specific considerations that must take precedence over considerations for cheap and fast education. Students need adequate opportunities to develop clinical competence, professional communication skills, and be socialized into the professional role in the clinical area (Cheek). Turley (1996) described a distinctly different type of concrete, action-based "praxis," knowledge that is similar to the "know how" a skilled craftsman develops through years of experience and fine-tuning. And yet, there are also numerous accounts in the literature that show it's not only possible, but desirable, to substitute some portion of clinical practice with CAI (Cheek et al., 1998; Kilmon, 1996; Theile \& Holloway, 1990; White, 1995).

\section{Expect Different Learning Outcomes}

Bates (1999) noted the primary advantage of CAI is that new and different learning outcomes can be achieved through the use of technology that need not imitate the goals and assumptions of either the classroom or clinical teaching arena. Many software programs are designed to provide the student working at a computer with more active participation in the clinical decision-making process than is possible in a real-life practice setting (Lyons \& Milton, 1999; Weis \& Guyton-Simmons, 1998). Computers can also minimize or eliminate many of the 
problematic variables that are commonly associated with the clinical practice area such as safety risks for both patients and students, time constraints, and limited opportunities to work with rare or unusual clinical problems (Gaberson \& Oermann, 1999; Weis, Guyton-Simmons, 1998). Anxiety associated with having an instructor watching your every move and that can severely interfere with a student's ability to concentrate and learn is often reduced with CAI (Cobb, 1999). In real-life clinical situations, students seldom receive corrective feedback which means many of their misconceptions go undetected and can result in serious harm. On the other hand, most CAI programs are designed to provide students with immediate, confidential, objective feedback that prevents them learning incorrect information and is often easier to accept and learn from than feedback from a human instructor (Cobb).

Lyons and Milton (1999) described a case-based computer program they used to teach nurse midwifery students clinical decision-making by providing them with intrinsic (constantly changing, computer-mediated) feedback as they examined a term pregnancy simulator mannequin that was connected to a multimedia program. Some of the benefits of the teaching mode for the teacher include the ability to control the level of difficulty of each teaching situation so that beginning students were not overwhelmed and more advanced students were not bored. Teachers were also able to provide a wide variety of learning situations for each student in the class at just the right time in each course which is something that seldom happens in a live clinical setting (White, 1995).

Weis and Guyton-Simmons (1998) investigated the utility of a CAI simulation program for providing students with opportunities to actively practice clinical decision-making. The program permitted free-text entry and the use of natural language to test the student's ability to 
solve simulated clinical problems. Some of the students $(\mathrm{N}=16)$, who were observed for patterns of behaviors as they interacted with the program, displayed computer anxiety but most of the findings were positive. Students used the immediate feedback about the actions they took to take corrective actions or make adjustments in a way that was similar to the way students learn in the real world. Observers noted the simulations allowed students to interpret data, make decisions, and develop systematic plans of action more often with CAI than would have been possible in a real life clinical situation. Students were forced to think but were allowed to make mistakes and learn from them. As would be expected, the findings showed students who were Licensed Practical Nurses (LPNs) and had previous clinical experience did much better than those without experience. Inexperienced students understood the factual information but were unable to interpret or evaluate the data and were not able to determine the impact their decisions had on the patient. All of the students' overall responses to the simulations were positive and most believed CAI was better at evaluating their knowledge that the typical multiple-choice questions used in the classroom (Weis \& Guyton-Simmons).

Many graphic simulations and virtual reality programs are designed specifically to teach students enrolled in dangerous professions decision-making skills. Gaberson and Oermann (1999) point out that nursing is a perfect profession to use CAI for training since it is characterized by complexity, instability, uncertainty, and unique and value laden-conflicts and problems. Because it is, software designers are increasingly creating life-like environments where nursing students can gain experience, make mistakes, receive feedback and go through the process of learning without risking lives. Just as pilots, air-traffic controllers, engineering students and surgeons have used simulators and virtual reality computers connected to 
mannequins for years, nursing simulators and VR programs are being developed to optimize the probability of the student making a correct decision in life-threatening situations (Abdulla, Henke \& Watkins, 1987; DeAmicis, 1997; Engberg \& White, 1998; Jeffries, 1999; McDowell, Nardini, Negley \& White, 1984; Merril \& Barker, 1996).

Several studies have shown that text-based case-study simulations and tutorial CAI programs are capable of teaching the types of higher level thinking skills students need to make clinical decisions. One study by Perciful and Nester (1996) used a combination of 4 simulations and 4 tutorial programs to measure the effectiveness of CAI for increasing knowledge and critical thinking abilities. The sample of 83 BSN students enrolled in a psychiatric nursing course were randomly assigned to one of two groups. The treatment group used the commercially available CAI software programs throughout (over four clinical days) and participated in a collaborative teaching model (student-faculty-service) during their initial clinical experiences. The comparison group participated in the "traditional" clinical experiences of rotations to mental health facilities and homework assignments. Knowledge was measured by the score for psychiatric nursing component of the Mosby Assess Test and two parts of the NLN Psychiatric Exam (theory and total scores). Critical thinking was measured by two parts of the NLN's Psychiatric Nursing Exam. The experimental CAI group scored significantly higher than the control group on assessing, analyzing, and evaluating, as well as planning and implementation measures for critical thinking skills. However, there was no significant difference between the two groups with respect to knowledge (Perciful \& Nester).

In a similar study, Pond (1987) used a pretest-posttest control group design to determine if a CAI program was more effective than traditional methods for teaching knowledge and 
critical thinking skills. The $28 \mathrm{BSN}$ students were randomly assigned to either attend a lecture or used CAI to learn the same content. Pretests included a 21-item multiple-choice exam and the Watson-Glaser Critical Thinking Appraisal. Posttest included comparable forms of the tests administered to both groups and a measure of attitudes toward the type of instruction. The scores for critical thinking did not improve significantly for either group but CAI was as effective as lecture for teaching course content and was well accepted by the students (Pond). In another, Budacki (1992) compared the effects of an interactive videodisc (IVD) program to teach associate degree nursing students decision-making when caring for postoperative patients. The study compared CAI and traditional lecture on cognitive achievement and affective response. The students $(\mathrm{N}=53)$ were randomly assigned to either the treatment or the control group after no obvious differences were found between the two groups in relation to age, gender, or previous computer experience. The results revealed no significant difference between the groups on cognitive achievement as measured by written examination scores but there was also no difference $(\mathrm{p}=.05)$ between the control and experimental groups concerning attitude toward method of learning (Pond).

Cox (1988) used a supplemental CAI simulation program to prepare students to administer intravenous (IV) medications to pediatric patients and found students in the experimental group administered the intravenous medications more quickly and more accurately than those in the control group (Cox). Thiele et al. (1986) tested a set of CAI programs to teach a group of 80 junior and senior nursing students the basic skills of decision-making. The results revealed significant improvements in accuracy in making decisions following completion of a series of five commercially purchased CAI software programs. The first tutorial program taught 
cue recognition and diagnostic reasoning and was followed by a series of four simulations programs to provide students with practice using the skills. Students attributed their successful outcomes to the immediate feedback and intense practice provided by the CAI programs that would have been lacking in the traditional clinical setting (Theile et al.).

\section{Expert Systems Support Clinical Decision-making}

Similarly, expert system software programs are being developed to support students and practitioners with decision-making tasks. Hanson, Foster, Nassah, Hodson, and Dillard (1994), a group of nursing faculty from Ball State University, developed an expert system program that provided clinical decision support for students and practicing nurses. The software was designed to support nurses in the identification and management of common post-operative complications. A formative evaluation of the system with ADN and BSN students elicited positive responses and formed the basis of ongoing program refinement. The educators emphasized the importance of nursing students understanding how to use the systems since such systems are clearly an upand-coming clinical tools that will be used by all nurses in the very near future.

Martin (2001) described an expert system software program that was designed to support family physicians and nurse practitioners in the management of a variety of minor outer eye disorders. The artificial intelligence (AI), or "knowledge" on the program, was gathered from the scientific literature and six experienced ophthalmologists and six experienced ophthalmic nurses. Another program described by Jirapaet (2001) was used as an alternative method of training and providing real-time clinical decision-making support for pediatric ICU nurses. The program was a multimedia interactive consultation-based program that contained two components: a knowledge base of nursing diagnoses and another of nursing interventions that 
were appropriate for the care of neonates on mechanical ventilators. The nurses scored their information access capability and clinical judgement as significantly higher $(\mathrm{P}=.0001)$ after the training session with the expert system.

In summary, the literature that addresses the ways CAI is being used to teach and provide students with practice making clinical decisions revealed that while most nurse educators recognize that an in vivo clinical setting is ideal but financial and practical considerations limit the amount of clinical practice the schools are able to provide for their students. There are also many benefits that can be gained from using CAI to replace some clinical time. Four different types of software are being used that are able to provide students with more independent simulated decision-making situations than a student would normally encounter in a live clinical setting. The use of computers can minimize safety concerns for both patients and students and improve learning by reducing students' anxiety levels. The provision of immediate feedback has been shown to improve learning and reduce the time it takes to learn. Benefits for the teacher include the ability to control the learning situation so that simulated decision-making situations match the students' ability and the content being taught in the course. Simulations and VR software programs are increasingly being used by nurse educators to teach a decision-making in a variety of specialty fields within nursing (Cheek et al., 1998; Kilmon, 1996; Theile \& Holloway, 1990; White, 1995).

Several studies that compared the use of CAI with traditional teaching methods for teaching clinical decision-making were discussed. Perciful and Nester (1996) compared CAI with traditional clinical assignments and found that the CAI group had significant improvements in several areas of critical thinking but similar knowledge gains. The Ponds (1987) study 
compared CAI with lecture for teaching knowledge and critical thinking and found students' scores for critical thinking did not improve significantly in either group but knowledge gains were similar in both groups.

Cox (1988) looked at the use of a CAI simulation program to prepare students to administer IV medications and found the students in the experimental CAI group were both faster and more accurate than the control group. Theile et al.(1986) used a combination of tutorial and simulation programs to teach clinical decision-making skills followed by intense practice and found significant improvements in the skills after the treatment.

Several authors reported expert system software programs are being used in nursing education with students (Hanson et al., 1994), to provide support for nurse practitioners caring for patients with minor eye disorders (Martin, 2001) and to train and support ICU nurses in the care of neonates on mechanical ventilators (Jirapaet, 2001).

The focus of the literature review will change to the primary topic of this study which is the integration strategies teachers use to integrate CAI into the courses they teach. This part of the review will include a brief discussion of instructional design theories and the literature that addresses the integration strategies under consideration in this study. For organizational purposes, the review will follow the same basic structure as the rest of the paper with a discussion of the various strategies falling under the broad headings of strategies to increase student learning, decreased course costs, students' time and the teachers' time.

\section{Strategies to Integrate CAI into Courses}

The strategies that were included in the "Integration of CAI Questionnaire" were taken from a text entitled, "Designing Effective Instruction" by Kemp, Morrison and Ross (2001). 
Most of the strategies were selected because they represent good teaching practices and were backed by instructional design principles. Others were selected based on recommendations or controversies surrounding them in the literature (Gustafson \& Powell, 1991). All 19 strategies have the potential to impact student learning and therefore, were included under the "student learning" section of the questionnaire. There is a great deal less information in the literature about how the integration strategies impact students learning time but many of the same strategies that are intended to increase learning have also been shown to reduce the time it takes for students to learn (Kulik \& Kulik, 1991). For that reason, and in an effort to make the literature review slightly less cumbersome, the two topics will be reviewed together. Then, the review will focus on the integration strategies that are primarily used to reduce course costs, and then the ones that are primarily used to reduce teachers' time.

Strategies for Learning

Instructional design theories provide a framework for systematically planning, developing and adapting instruction based on identified learner needs and course content requirements. There are many instructional design theories (Alessi \& Trollip, 1985; Briggs, 1991; Dick and Carey, 1991; Gagne', 1985; Merrill, 1983; Reigeluth \& Schwartz, 1989) that are used by teachers to guide them in designing an entire course or by instructional designers to design a single unit of instruction (Kemp et al., 2001). Gustafson and Powell (1991) reviewed many of the major theories and found that while the terminology is different, for the most part, there are very few fundamental differences among them. A simplified version will be used in this review that is based on Gustafson and Powell's summary and terminology.

Pre-instruction strategies. The first four integration strategies of the Integration of CAI 
Questionnaire are actions or decisions the teacher typically makes before the instruction begins. The first is to "pre-test" learners to be sure they are adequately prepared to learn the content of the CAI program (Kemp et al., 2001). The purpose of the pre-test is to ensure the CAI program closely matches the needs and abilities of the students who will use it (Dick \& Carey, 1991). The underlying principle is from a number of instructional design theories that have their roots in General Systems Theory (GST) (Bertalanffy, 1968). According to the theory, a system is an integrated set of elements that dynamically interact with one-another (Gustafson \& Powell, 1991). Rather than a piecemeal approach, systems theory advocates strive for congruence between learners, objectives, instructional methods and evaluation procedures. That means that before a CAI software program can be selected or designed, an empirical needs assessment must to be conducted to insure the instruction is relevant and that there is a close match between the various parts. Relevance means providing exactly the right instruction so that a specific group of learners are able, at the conclusion of the instruction, to perform the skills of the job (Gustafson \& Powell).

Khoiny (1995) reviewed the nursing literature to see what factors impacted student learning when using CAI and found that (1) the quality of the software, (2) the environment where the computer is used and (3) the characteristics of the learners were the three most important factors. Because the quality of commercially available CAI nursing education software varies greatly, she suggested the teacher should select programs that are affordable but of the highest level of quality possible. Commercially available software should be tested before it is purchased to be sure it contains correct information, has clear instructional objectives, correct sequencing, and appropriate and immediate feedback (Khoiny). Dick and Carey (1991), 
a pair of instructional designers, stress the importance of selecting programs that are based on sound pedagogical principles and thoughtfully selected instructional strategies. The highest quality programs begin with prerequisite memory-level knowledge but quickly advance to teach performance-level thinking skills. Poor quality programs teach at the memory level only and fail to support the learner in achieving performance-level objectives. In higher education courses, performance-level objectives should be introduced in early courses and continued throughout the entire curriculum (Dick \& Carey).

Gleydura et al. (1995) also noted the use of poorly designed software or the inappropriate application of software not only detracts from the educational objectives of the course but will end up wasting students' time and could turn them against using the media in the future. Conklin (1983) pointed out that if CAI applications are to be effective, nurse educators need to be aware of which types of software are most effective for meeting different learning objectives and select the most appropriate programs based on the objectives and the readiness of the students in the course. Goyne, McDonough and Padgett (2000) noted several practical guidelines evaluators need to consider are whether or not the software provides a variety of appropriate media and activities, supplies formative feedback and achievement measures, allows opportunities for practice and review and is both enjoyable and interesting. Two of the most important criteria, according to Bowell (1995), are whether or not the program is interactive and do learners become intensely engaged in the instruction? Interaction means the learner can apply, try out or practice what he/she is learning as opposed to simply clicking on a button to turn a page. The person evaluating the software needs to determine if the software permits social interaction so that it can be used for small-group or whole-class learning. Also, is the time it 
takes to learn the content comparable to the amount of time it would take using a traditional teaching method? Can the school afford to purchase enough copies to make the software available to the number of students in the class (Bowell)?

In addition to ensuring that the students are academically ready for the software, it is also important to match the level of difficulty of the software with the computer skills of the students in the course. Cobb (2001) videotaped groups of nursing students as they interacted with an IVDI program and found the majority of students without previous IVDI experience had difficulty accessing and proceeding through the lessons. Fill-in-the-blank questions posed by the software were often frustrating and students had difficulty reading the touch screen monitor. One of the conclusions she reached at the end of the study was that the students were ill-prepared for the assignment, that they needed more orientation to the computer equipment and faculty needed to be certain that technical assistance was available throughout the lesson for students who needed it (Cobb).

According to the literature, the second through the fourth integration strategies are primarily implemented by teachers to improve learning and decrease students' learning time. The strategies are to "explain the objectives, explain how to use the CAI program, integrate the CAI content with the rest of the course and motivate students by explaining the value of learning the content of the CAI" (Kemp et al., 2001). The first strategy is taken directly from instructional design theory that states the goals and objectives of instruction should be clearly stated and understood by the learners beforehand (Gustafson \& Powell, 1991). Goals are broad statements of instructional intent (e.g., provide care for a post-operative patient) while objectives are specific steps leading to the attainment of the goal (e.g., assess the patient's intra-cranial 
pressure). The focus of the instruction, according to instructional design theory, is always on the learner, which means the goal is always a change in learners' knowledge, skills, or attitudes (Willis, 1993).

All three of the strategies relate to Gagne's Nine Events of Instruction (1985) which are activities carried out by the teacher and learners before or during the instruction. Gagne's model is based on information processing model of human cognition and the internal mental processes required for learning. The processes include reception, semantic encoding, response, organization, and retrieval.

The first event of instruction is to gain the learners' attention or help the learner become more receptive by focusing his/her attention on the learning tasks. This may be accomplished by presenting an unusual or discrepant event (a problem, story, or illustration) or for adult learners, attention can be gained by presenting words or descriptions that relate upcoming events to something that is important to them such as the objectives of the program. The purpose is to help the learner focus his her attention and sort out important from unimportant material and thereby, save their time and energy. In order to increase the relevance of the instruction, the goals and objectives of the instruction, even when they appear in printed form in the instruction, should be pointed out verbally by the teacher before the instruction begins so that students come to the instruction with their attention and study efforts clearly in focus (Gustafson \& Powell, 1991). A direct statement of the objectives is usually sufficient for adult learners (Gagne'). Attention also must be maintained throughout the lesson for learning (semantic encoding) to occur. This is best accomplished by the teacher (or CAI) through meaningful interactions and by presenting the content in a way that engages and challenges the learner mentally (Gagne') . 
The thinking behind the strategy of demonstrate or "explain how to use the CAI" program beforehand is to prepare learners for what to expect, to decrease the time it takes to begin learning and to prevent their being distracted from learning by peripheral concerns such as how to operate the computer or navigate through the program (Khoiny, 1995; Perciful \& Nester, 1996). Brudenell and Carpenter (1990) noted a lack of orientation to the computer and software program as possible reasons that students have negative attitudes toward CAI. After observing hundreds of students struggling to complete assignments in a library computer lab, MacKeller and Elliott (1999) wrote to warn teachers in higher education that just because the label on a CDROM package says the program is user-friendly it does not mean that the teacher does not need to prepare students beforehand and make sure students have both technical and instructional help available.

When working with CAI, prior computer-related experience has been shown to be important. A sample of both undergraduate and graduate students enrolled in Web-based courses identified a lack of both computer skills and access to necessary computer equipment as potential barriers to learning in the program (Theile, Allen \& Stucky, 1999). Similarly, observations of nursing students completing CAI programs identified an association between lack of computer skills and the increased likelihood that the user would be unsure how to complete the assignment or would be become easily frustrated (Weiss \& Guyton-Simmons, 1998).

The next integration strategy is to "illustrate the relationship between topics in order to integrate the CAI with the rest of the course" (Kemp et al., 2001). The purpose is to increase students' motivation to learn by helping them understand why the content is important, how it 
relates to what they already know or to the rest of the course and how it will be of value to them in the future (Gustafson \& Powell, 1991). The strategy also correlates with Gagne's (1985) Third Event of Instruction which is to present a stimulating recall of prerequisite learning. The assumption behind the strategy is that all learning builds on what is already known which is particularly true when teaching complex, integrative skills such as clinical decision-making where learners need to have relevant prerequisites in their minds in order to integrate them with new ideas. According to Gagne', simply presenting the information may not be sufficient, learners may also need to take some action with the prerequisites in order to bring them into active memory.

Lyons and Milton (1999) describe an excellent example of how a group of Australian university faculty integrated a virtual reality program by linking it with the theory component of the nurse midwifery course. The Pregnancy Simulator Learning Package (PSLP. ) was used in conjunction with didactic instruction in much the same way as a clinical laboratory learning experience might be used. Students conducted a physical examination on a mechanical model of a term pregnancy abdominal simulator that was linked to a computer model of a hierarchicallystructured interactive learning environment that presented a range of simple-to-complex learning experiences embedded in a group of problem-led case scenarios. The case studies provided situated learning experiences that were intended to reflect the way theoretical knowledge is used to care for women in real world situations. Some of the case studies were designed specifically to address misconceptions that students might hold about the physical assessments. The primary advantage of the PSLP was that it provided students with ready access to the learning experiences so they were able to practice immediately after learning the new information and 
still had the prerequisite knowledge in mind when asked to apply it. Students were also encouraged to repeat the learning activity as many times as needed in a non-threatening, nonhurried environment until they achieved mastery. Another important advantage was the immediate intrinsic and extrinsic feedback the simulator provided. Privacy and ethical considerations often prohibit instructors from providing the immediate feedback students need during an actual bedside assessment teaching session. Instead, students are often given feedback in another room at a much later time which reduces the opportunity for learning (Lyons \& Milton).

In the Thede et al. (1994) study, the 12 graduate and undergraduate nursing students who were interviewed believed that faculty should have done more to integrate the CAI into the course. Many thought a simple explanation from the teacher would have gone a long way in preparing them for what to expect and for motivating them to learn the information. Students also said they needed to be warned there were embedded questions within the CAI that would be helpful for the test (Thede et al.). On the positive side, several students said completing CAI programs before coming to class helped them prepare for the class, and as a result, their participation in the classroom discussion increased and was more meaningful. The researchers concluded that if teachers have as their goal to improve the quality of education with CAI, rather than just providing automated instruction, they need to change their role from an information provider to a facilitator of the instructional process. This means they need to provide students with support before, during, and after the instruction (Thede et al.).

Modes of instruction. The mode of instruction the teacher selects has also been shown to impact both student learning and learning time (Kemp et al., 2001). Khoiny (1995) noted that 
there are a large number of complex variables the teacher needs to take into consideration when making the decision about which mode is best such as course objectives, types of computer hardware, classroom facilities, students' computer skills, the number of students in the class as well as the teachers' level of comfort and expertise with computers (Khoiny). Gagne's (1985) fourth Event of Instruction is "present the facts, knowledge or skill to learners in a context or background." The context or background of learning may be individualized, a small-group or a classroom setting where the teacher uses a multimedia simulation or virtual reality program to present the lesson to the whole-class(Wells \& Kick, 1996). Regardless of the mode of instruction, the goal during this event is always to help the learner focus their attention on the relevant parts of instruction. This can be accomplished by the teacher (or the CAI program) using attention directing techniques such as simplifying the display, using voice inflection, underlining, circling or pointing at, or enlarging the display. The presentation may be interactive with questions, answers, and activities that must be completed correctly before the student advances through the material or the teacher (or the CAI program) may use a model or show the learners what a correct performance looks like or what constitutes a correct response. Other options might be to illustrate or show all the steps to complete the problem or provide guidance or suggestions (Gagne’).

Once the presentation is complete, the Fifth Event of Instruction of Gagne's (1985) model is to elicit the actual performance or provide the learner with an opportunity to practice. During this event, the teacher (or the software program) turns the action over to the learner to see what they do, and do not, know. The emphasis at this phase is on learning and not testing (Gagne, Briggs \& Wagner, 1992). One of the major advantages most often cited in the literature 
of CAI is the high level of interactivity and attention it requires of the learner. Interactivity means two-way communication between the user and the computer in a way that fosters the learner's active involvement and stimulates thinking. Wittrock (1991) noted that in order for learning to occur, learners must, at minimum, be mentally active in the learning process. This may or may not happen, or may intermittently happen, during small-group or classroom instruction or while reading a textbook, listening to a lecture, or participating in a discussion but mental activity is more likely to be sustained when the learner is required to provide the correct responses to questions before advancing to the next level (Wittrock). A popular term in education is "active learning" which implies students learn best by "doing" or are directly engaged in the application of content (Epper, 2001). Many of the newer CAI software designs teach both content and process at the same time when they allow students to experiment with and manipulate variables or simulated equipment and instantly see the results (Epper).

From its inception, CAI was designed to promote individualized learning and to adapt instruction to each student's needs in much the same way a tutor would provide one-to-one instruction. Many authors refer to the use of computerized instruction as a one-student-onecomputer activity (Johnson \& Johnson, 1985). An important feature of individualized CAI is that it makes it easier and more convenient for learners to access the instruction, which in and of itself, may contribute to better learning outcomes and save valuable time (Bates, 1999). Many CAI software applications are being used in ways that are not new but are merely new applications of old methods that have been made more accessible. For instance, many text and reference books are being replaced with CD-ROMs and DVDs. Learning takes less time because the media has search capabilities which textbooks do not have. In addition to text, CD-ROMs 
and DVDs have such large storage capacities they are able to provide almost unlimited and instant access to sophisticated graphics, animations, sound and video so that students are able to watch and hear a procedure or treatment being performed by an expert instead of reading about it in a textbook and trying to visualize what it might look like (Gleydura, Michelman, \& Wilson, 1995). The search, pause and replay capabilities allow students to repeat the presentation until they are ready to advance to the next section which also saves the learner's time (Laurillard, 1993).

As anyone who has ever struggled to learn from haphazardly arranged information knows, a reduction in the students' learning time is conditional and depends on the way the instruction is designed and presented (Gibbons \& Fairweather, 1998). The packaging of the instruction makes a large difference in terms of learning efficiency as well as the energy and motivation of the learner. Each of these variables, in turn, contributes to how much is learned and the depth of learning. In other words, more learning can be accomplished in a given amount of instructional time if the developer has arranged things to make that happen (Gibbons \& Fairweather).

There is a trend in software design toward even more individualized instruction. Dick and Carey (1994) define instructional design strategies as the activities software designers use to help the student accomplish the performance goal. Many of the best programs contain, not just one, but a sequence of instructional strategies that can be selected, applied, assessed and discarded until one is found that works. These programs use artificial intelligence and multimedia to dynamically adjust instructional strategies to make CAI an adaptive instructional experience in which strategies are adjusted according to how the student responds to the 
instruction. For instance, in a multimedia environment, if speaking words doesn't work, then perhaps showing a film will, if one type of practice doesn't help, then perhaps another will. Gibbons, Fairweather, and O’Neal (1993) also explained instruction used to be perceived as an “informing" activity but CAI inquiry support tutorial programs are being developed that have changed how learning occurs. The learner (as opposed to the teacher) assumes a greater amount of responsibility for learning but they are aided by an observant support system that is capable of monitoring, learning, coaching, advising, judging, providing feedback and assisting with the selection of the most beneficial learning paths so that students can carry out progressively challenging real-world tasks. Learning with these types of programs can be done in much less time than in a traditional classroom or by using a textbook (Gibbons, Fairweather, \& O’Neal).

There are many advantages for students working independently on a CAI assignment. Smith, Jones and Waugh (1986) noted the major advantage of CAI for a college-level chemistry class was the self-pacing feature that permitted lessons to be tailored to the level and learning style of the individual learner. Conklin (1983) noted that various academic backgrounds and learning styles could only be addressed through individualized instruction. Levin (1983) also found the learner's interest, rate of learning and knowledge-base were acknowledged and supported through individualized instruction. There is considerable research that has shown that students working independently with CAI demonstrated a marked improvement in motivation and enthusiasm for education, the development of critical thinking, problem-solving and a greater capacity for recalling information (Charp, 2001). Scanlan (1986) noted that the convenience of learning any place, any time makes CAI an attractive medium that is particularly well suited to adult learners (Knowles, 1984; Reif, Roulis, \& Huber, 1995). Since adults’ 
readiness to learn is motivated by reality and practicality and their interests tend to focus on problem-solving rather than on abstract content or theory (Knowles), many adults like learning from computers because computers alleviate the tedious activities of memorization and hunting for information and they allow the learner to plan their own learning projects, control the pace of instruction, decide when the content is presented and select the style of the presentation. The fact that adults learn well from computers was confirmed when Roblyer (1988) reviewed the research on the effectiveness of microcomputers for educational applications by comparing results across age groups and found those with the best results were the college and adult-learner populations.

A completely independent home-study CAI program on pharmacokinetics was tested by 27 APN students enrolled in a traditional lecture continuing education course in pharmacology (Neafsey, 1997). The APNs took a knowledge test before and after using the CAI program which showed all 27 had significant knowledge gains. The students cited convenience, organization and simplicity of use, graphic animations, and interactive question sequences as advantages of the program. Home study was found to be a feasible option to printed home-study and a lecture-based continuing education program in this study (Neafsey).

As the student population of higher education becomes increasingly diverse, the selfpaced format and unlimited practice potential of CAI could become an important way to provide instruction for students who require different amounts of time and practice to accomplish the learning objectives (Lerner, Cohen \& Brown, 1990). Gaberson and Oermann (1999) noted it is common for some nursing students to be able to perform in a few hours what it takes others the entire clinical day to accomplish. Individualized assignments can save the more advanced 
students time while providing slower students with the opportunity to go at their own pace just as they do in the clinical setting (Gaberson \& Oermann).

Strategies to Decrease the Students' Time

Substantial reductions in the time students spent learning with CAI have been reported in several studies (Bitzer \& Boudreaux, 1969; Larson, 1982) with secondary and post-secondary students (Kulik, Bangert, \& Williams, 1983; Kulik, Kulik, \& Cohen, 1980). Tilson (1986) found students working independently at a computer spent $50 \%$ less time to learn the same content as students who attended traditional classroom instruction. Not only has CAI been shown to reduce the amount of time it takes for individuals to learn (Huckabay et al., 1979) but it has also been shown to reduce by one-third (Bowell, 1995) to two-thirds the time needed for a conventional course (Kulik \& Kulik, 1991; Clark, 1991; Reynolds \& Pontius, 1986). Still other studies have shown the opposite effect and that CAI motivates students to invest more time in the subject-area which ends up increasing their time to learn. This was especially true when the student is free to work at his/her own pace and develop their own time schedule (Worthen, Van Dusen \& Sailor, 1994). Even when students used older text-based programs, most studies indicated that students learned as much information more quickly. Estimates of time savings vary considerably and range from small reductions to findings such as the Bitzer \& Bordreaux (1969) study, mentioned earlier, who found the CAI group learned equally well as the lecture half of the class but cut their learning time from 66.5 to 23.5 hours.

In Hmelo’s (1990) review of the medical, nursing and allied health literature over a 20-year period, the findings showed no significant difference between the CAI and the control groups on performances but there was a significant difference in favor of CAI for decreased 
learning time (Hmelo). Different authors attribute the time savings to different reasons. Bitzer and Bordreaux, 1969) thought it was due to the learners' ability to pick and choose the content that is needed and skip portions that are not while others believed it was due to the fact that the instructional designer spent considerably more time designing and arranging the instruction than a classroom teacher was able to spend. Better design and organization (Bunderson et al., 1984; Clark, 1983; Kulik \& Kulik, 1991; Kulik, Kulik \& Cohen, 1980) and more individualized interaction and feedback were also thought to contribute to decreased time to learn (Bates, 1995a).

One particularly innovative example of using CAI to substantially reduce students' time to learn was reported by Bareford (2001) who used an inexpensive commercially available computer simulation game called Sim City to increase student learning and reduce course costs by reducing the amount of time the teacher spent and dramatically reducing the amount of time students' normally spent on the learning task. The simulation was used as a supplement to a community health course along with traditional lecture-discussion. Students worked independently with the CAI simulation using teacher designed problem-solving modules developed for the course. Post-instruction activities included a debriefing meeting held immediately following the CAI activity and one week later at a post conference. Students were enthusiastic about the CAI experience and most had a better grasp of the problems and a deeper appreciation for the complexity of community health issues. The simulation allowed students to learn as much in several hours as it would normally have taken several weeks of work to learn in a traditional graduate level community nursing course (Bareford). 
Modes of instruction. Vincent's (1984) dissertation study compared an individualized mode of CAI with lecture-discussion for effectiveness and efficiency. The sample of $(\mathrm{N}=35)$ junior BSN students were randomly divided into two groups both of whom received instruction on the same day at different times. There were no significant differences between the ages or GPAs of the two groups. A paper and pencil test was administered on the third day after the instruction and an unannounced test was administered four weeks later and the time spent on instruction was documented for both groups. Analysis of test scores revealed no significant differences in student performance on the initial post-test score but the delayed post-test showed the computer learners' scores were significantly better $(\mathrm{p}<.05)$ than lecture learners' scores despite the fact that the computer learners spent 18\% less time on the instruction (Vincent).

There are a number of studies that have shown there are advantages and disadvantages to assigning students to work on CAI assignments in small groups. Small-group CAI instruction refers to students interacting to accomplish shared goals while ensuring all group members master the material (Johnson \& Johnson, 1985). The primary reason teachers use CAI for smallgroupinstruction is to provide students with opportunities to practice collaboration and negotiation skills in order to prepare them to work as members of a team. Other reasons are to save time, to accommodate shortages in computers or software programs, to provide academically weaker students with peer support or to ensure novice computer users are not left behind (Cobb, 1999).

Belfry and Winne (1988) noted that if educators are to make the most of CAI, they need to select the type of programs that are most suited to the course and appropriate for meeting different learning objectives. If the course objectives call for students to learn how to 
collaborate with other professionals in making clinical decisions then the teacher needs to select software that supports a small or large group mode of learning or at least one that presents problems that support collaborative decision-making (Belfry \& Winne).

There is still limited research available on the learning effectiveness of CAI with small and large group instruction (Calderone, 1994; Webb, 1985; Weiss \& Jarvis, 1986-87). Bejarano (1987) found a small-group presentation was effective due to the social interaction and active participation of team members. Wizer (1987) also proposed the exchange of ideas among members of a small-groupfacilitated problem-solving. It was the process the small-groupengaged in rather than coming up with one correct answer that was advantageous to the learning process (Wizer). Bouton and Garth (1983) suggested that there were two major elements that needed to be present for small-group learning to be effective; the first was to structure the tasks under the guidance of the teacher and the second was to place the emphasis on peer interaction and dialogue to construct knowledge (Bouton \& Garth). To promote interaction among groups of students using IVDI, Miller (1990) required a group consensus before the group could type an answer into the computer. The use of IVDI with small groups (2 to 3 ) of law students fostered consultation among students, strategy making and the sharing of information (Miller). Castner (1992) examined the cognitive strategies used by nursing students using a computer simulation program to identify a nursing diagnosis working alone and in dyads. The results of the qualitative study indicated students collected data, coalesced clues into clusters, activated possible diagnostic explanations, did hypothesis and data-directed searches of the data field, tested diagnostic hypothesis for goodness of fit, and labeled. The dyad groups of students did more exploring that students working alone but there were no apparent differences in strategies 
and both groups accomplished the course objectives (Castner). Watson (1966) identified some of the factors that facilitated and impeded group performance while students worked on CAI assignments. Some of the facilitating factors included the following: social stimulation; multiple resources that could be contributed by each member; the probability of having members with higher ability and the motivation to provide insight to others in the group; the rectification of individual blind spots; a sense of security in risk-taking, and the canceling of chance errors. The impeding factors included the following: lack of unity, frustration encountered with interference and criticism, rambling talk by some participants, leaving the tasks for others to accomplish, a lack of motivation, opposing goals, and failure to communicate with other members (Watson).

As noted above, a significant drawback to group learning is unequal contribution by group members which can foster resentment and undermine the learning process (Simpson, 1986). Slavin (1983a) identified the problem as a "diffusion of responsibility" in which individuals were rewarded for a minimum contribution that eventually led to diminished performance by all members. In a follow-up study, Slavin (1983b) addressed the concern for equal member participation by promoting group rewards that depended on each member's academic performance. Individual accountability was fostered through a sense of responsibility for the accomplishment of the group goal. Group success was ascertained by a sum of the members' learning performance (Slavin, 1983b).

There are a number of suggestions and problems encountered when using a group mode of CAI instruction discussed in the literature. For instance, Khoiny (1995) suggested problems with insufficient computer resources (computers and software) could be overcome by dividing a large group of students into smaller groups that are assigned to use the computers at scheduled 
times. Perciful and Nester (1996) noted students who used clinical simulations as a group encountered problems because some students completed the assignment more quickly than others; some took less than a hour while others took over 2 hours. The time difference made it difficult to hold a post-simulation group discussion. The researchers recommended simulations be completed independently and then schedule a follow-up discussion time that was agreeable to all (Perciful \& Nester). A study by Koch, Rankin and Stewart (1990) revealed most of the students in their study preferred to use a small-group mode over an individualize mode.

Baird and Koballa (1988) examined students' perception of success as well as the effects of mode of computer instruction and group size on the acquisition of hypothesizing skills among undergraduate students. Two types of computer instruction were used; simulations and textual exercises. The subjects were 87 elementary education majors who completed one mode of the CAI program individually or in groups of 3. The data analysis revealed the highest mean of perceived success (4.65) was acquired by students using simulations in groups and the lowest (3.28) was by individuals using the textual exercises. Software ratings were higher for groups than for individuals $(\mathrm{p}<0.005)$. Thus, student perception of success as well as attitude toward the instructional program may be influenced by the type of CAI program and the mode of instruction (Baird \& Koballa).

Cobb (1999) investigated the use of IVDI and cooperative learning strategies by assigning BSN students to one of three groups based on their pre-nursing grade point averages (GPAs). The groups were composed of homogeneous average achievement, homogeneous high achievement and heterogeneous mixed achievement groups of between three to five students. Students were videotaped as they viewed and interacted with an interactive video disc instruction 
(IVDI) program on cardiac assessment techniques. There were no significant differences among the groups in their interaction frequencies, cooperativeness, or achievement on post-test scores that were completed immediately after the lesson. Homogeneous high achievement students scored significantly higher than the other two groups on individual post-tests given two weeks after viewing the lesson. However, all three groups had very low scores (Cobb).

Noell and Carnine (1989) explored instructional effectiveness and efficiency when delivering IVDI to individual students $(n=14)$ and groups with an average of five members $(n=19)$. The results of an evaluation of 18 short-answer test items indicated an average score of 8.9 for the group mode and an average score of 9.0 for individuals. There was no significant difference in achievement between individual learners and groups of learners. Learning time was 40 minutes for a group of five students and 30 minutes for an individual, which meant five individuals would require 150 minutes to complete the assignment. The implications of the findings suggested the group mode of instruction was more time-efficient but did not result in a loss of achievement (Noell \& Carnie).

In another study, Park (1993) examined the effects of cooperative and individual learning with CAI with students enrolled in a university level introductory chemistry course. The sample consisted of $(\mathrm{N}=109)$ subjects who were assigned to one of four cells in a two factor experimental design. The two factors were learning group and learning ability level. The two groups within the learning group factor were cooperative groups and individuals working alone. The two factors within the ability groups were high and low ability. All participants worked a minimum of one hour a week after attending the same lecture. Subjects who participated in cooperative learning outperformed subjects who worked independently. High-ability students 
and low ability students working in cooperative groups improved in their performances more than high ability and low ability students working alone. There were no significant differences on students' attitudes between those who worked in groups and those who worked alone (Park).

Whyte (1990) examined the effectiveness of cooperative computer-assisted learning groups based on learning styles (field independent, field dependent or mixed). Out of 86 students, 57 were randomly assigned to the paired $\backslash$ cooperative treatment and 29 were assigned to the individualized treatment. The design was a quasi-experimental, three group, posttest only group design. There were no significant differences between the mean posttest scores of subjects who worked individually and those who worked in pairs. However, the manner in which subjects were paired made a significant difference in individual achievement test scores. Groups made up of either two field independent students or mixed groups of one field independent and one field dependent student significantly outperformed groups made up of two field dependents. Although field-dependent learners enjoyed working in pairs more than any other group, the optimal pairing included one field independent student. Field independent students performed equally well regardless of their partner but field dependent students benefitted significantly when paired with a field independent student (Whyte).

A common theme of student interaction in whole-class CAI collaborative learning has been examined by several researchers. Collaborative learning is described as learning that occurs through active participation by teachers and students (Hiltz, 1990; Whipple, 1987). As with the small-group mode, knowledge is thought to emerge through student-to-student interaction instead of through the delivery of subject matter content by the teacher (Simpson, 1986). Observations of students' activity during computer use demonstrated more social interaction 
when attention is directed at attaining educational goals. However, group-learning involved more than the grouping of students to accomplish a task. Teachers were needed to facilitate and ensure group productivity (Simpson).

Kilmon (1996) described using whole-class CAI instruction to teach beginning nursing students decision-making and problem-solving skills. Students were assigned to complete the CAI lesson on their own outside the class. Then, class sessions using the same commercially purchased programs were used to stimulate whole-class discussion of public health implications, ethical issues, or other themes that were relevant to the specific set of cases assigned. The teachers also reviewed case studies to guide students in collecting objective and subjective data, problem solving, to suggest management strategies and elicit class discussion on the various treatment options (Kilmon). Goodman and Blake (1996) describe a team of 6 nursing teachers who designed a set of CAI multimedia programs for in-class exercises with interactive case studies and short scenarios. The class, as a whole, made decisions and experienced the consequences when wrong decisions were made. The "traditional classroom where students passively receive information had become a participatory interactive environment in which students were actively involve"(p. 293). An evaluation of the software revealed students valued interacting with peers at the computer and enjoyed working in groups. The CAI software was valuable for testing students' ability to articulate concepts and provide a rationale for their decisions. Measurements that were used for the summative evaluation of the project included the students' evaluations of the courseware and their performance on exams and course grades. 
Each score demonstrated the software was effective for assisting students in meeting the objectives of improved mastery of course content and decision-making skills (Goodman \& Blake).

Technical and instructional support. In addition to mode of learning, two other integration strategies were included under the heading of midinstruction strategies. The strategies were to provide technical and instructional support. The decision of whether or not to include the costs for "technical and instructional support personnel" in the course budget (Kemp et al., 2001) is primarily a cost issue but has also been shown to significantly impact both student learning and time to learn. Cambre and Castner (1993) noted teachers who used CAI identified the costs of purchasing hardware and software as their major concern but their second biggest concerns were the troublesome technical problems with both hardware and software. Teachers stated, "they knew they were going to happen, it was just a matter of how long it would take to get them fixed and how disruptive it would be to the course" (Cambre \& Castner, p. 28).

Bates (1995a) explained teachers need to consider access, and not effectiveness, when they are deciding which instructional delivery system to use. Access includes more than just supplying students with computers. Teachers who plan to use CAI need to pay close attention to details such as how students will gain access to the computer laboratory and software programs, how they will be oriented to the computer and each new software program and who will handle technical problems when they occur. Access has become a much more complex issue as the diversity of the student body in higher education has increased (Bates, 1995a). Typical college students are no longer traditional 18 to 22-year-old on-campus students but are increasingly parttime adult-learners who need to access CAI from home, the workplace, or a remote computer 
center located some distance from the campus. Even when students purchase their own computers, the teacher still needs to ensure they have access to technical and instructional support over the phone, over the Internet or some other way. Factors that need to be taken into consideration when deciding how much and what types of technical and instructional supports are needed are things like how flexible the software is for students with different educational backgrounds, incomes, ages and prior learning and computer experience. When trying to decide if technical support is enough, or whether students will also need ready access to instructional support personnel, depends on all of the above factors as well as the user-friendliness of the particular CAI program, the complexity of the course content and the objectives of the course (Bates).

The importance of having adequately trained and well-equipped personnel available at the computer lab is a common theme in the literature. For instance, Lowdermilk and Fischel (1991) noted students experienced frustration from lost time traveling long distances to computer laboratories and once there, having problems because computers and software were not available. Weis and Guyton-Simmons (1998) observed students as they interacted with a CAI simulation and noted those who lacked previous computer experience appeared very unsure and hesitant and those who lacked typing skills became easily frustrated. Some students who were only given written instructions without an explanation or assistance couldn't even formulate a response. Many students said it would have helped to have the computer and the software program thoroughly explained before they started or to have someone available to help when they didn’t know how to proceed (Weis \& Guyton-Simmons).

Several authors provided suggestions about how to overcome problems providing 
students with CAI services that would also, under the right conditions, eliminate the need for technical and instructional support personnel. Khoiny (1995) suggested nurse educators could overcome access problems by having students purchase their own computers or have the school purchase mobile laptop computers that could be used whenever or wherever students needed them. Another suggestion was to provide access to technical and instructional support personnel over phone lines or the Internet more cost-efficiently. One of the ways schools deal with shortages of software are to provide access to CAI software programs over a campus-wide network, or the Internet (Khoiny). McGinty, Reed and Sautter (1987) noted many schools were purchasing software programs designed to run on networks of computers linked to a central file server. That way the schools are able to eliminate the need for individual copies of software and ensure the safety of the discs by storing them in a locked central location (McGinty et al., 1987).

Several authors explained the need for nurse educators to either provide or make sure someone else provides an adequate orientation for students who don't know how to use computers or new software programs as they are added to the course (Bates, 1999; Khoiny, 1995; Weis \& Guyton-Simmons, 1998) ). It is equally important that the teacher ensure there are properly trained, knowledgeable resource persons available at the computer laboratory who are willing to answer questions and help students resolve problems that may arise (Khoiny). Thede et al.(1994) noted a major hurdle students in their study identified was a lack of knowledge of how to use computers or the software. They reported problems such as long waiting lines to use a computer, programs that were not user-friendly and issues with compatibility between software and hardware. The researchers attempted to resolve the problems students had with the unhelpful technical and instructional support personnel by providing the laboratory assistants 
with retraining and sensitivity training sessions as needed (Thede et al.).

Post-instruction strategies. Two post-instruction integration strategies intended to increase student learning were included in the CAI Questionnaire. The first was to conduct a "post-instruction" discussion or critique to reinforce what students learned and correct students' misunderstandings" (Kemp et al., 2001). The strategy relates to Gagne's (1985) Sixth Event of Instruction which is to provide learners with feedback about the correctness or incorrectness of their performance. According to Gagne', the teacher (or CAI program) should explain the level of accuracy of the students' performance and why some answers are correct and others are not. The sooner the feedback is provided, the better and the more precise and individualized it is, the better the learning outcomes (Gagne').

Lange, Haak, Lincoln, Thompson, Turner and Weir (1997) evaluated a computerized program's ability to teach diagnostic reasoning to nurse practitioner (NP) students. Based on the responses of nine volunteer students successful completion was not based on prior computer experience because students were provided with an orientation to the computer hardware and software prior to the learning experience. The students recommended a conference or seminar with a live instructor following the CAI exercise to provide them with an avenue through which they could integrate the CAI learning with other learning experiences (Lange et al., 1997).

The next postinstruction integration strategy is to provide "follow-up activities to ensure students use what they learned in the CAI program." It is also closely related to the Eighth Instructional Event of Gagne's model and is also intended to enhance retention and the transfer of knowledge. Transfer is the ability to use the new skill or knowledge in a variety of applications or to be able to apply the knowledge in settings and situations other than those in 
which it was learned (Anderson, 1990). Both postinstruction strategies relate to Gagne's Ninth Event of Instruction which is to provide a review or a summary of the learning. This can be done either verbally for the adult learner or through follow-up learning activities (Gagne'). In a CAI program, the responses may be as little as "correct" or "incorrect" or as elaborate as hints, explanations, or suggestions. Generally, the more informative the feedback the better the quality of the program. For instance, Thede et al.(1994) found the students in their study students reported they liked informative and immediate feedback and noted the quizzes at the end of every section were extremely helpful. On the other hand, software that limited feedback to "correct" or "incorrect" was not perceived to be helpful and the students thought the feedback should have corrected their misconceptions. Other students noted using CAI before attending class helped them prepare for the discussion and helped them more fully understand the difficult concepts presented in the lecture (Thede et al.).

The two postinstruction integration strategies of "include the content or skills to be learned from the CAI program on the course exam" and the one that immediately follows it on the ICAI Questionnaire "include scores earned on tests embedded in the CAI as part of the course grade" can be used by teachers either to increase learning or to reduce the time the teacher spends on tests (Kemp et al., 2001). As mentioned previously, the rationale for both strategies is to motive students to do the assignment and help them view the CAI assignment as an integral part of the course and therefore, deserving of the time and energy it takes to learn the content.

Both strategies are closely related to Gagne's Seventh Event of Instruction which is to assess the learners' performance by having them do a complete demonstration of the learning 
outcomes without assistance, prompting or guidance. According to the model, if the objective says, "demonstrate," then the learner should be asked to perform the task and not asked to describe how to perform or to answer questions about how something is done. Gibbons and Fairweather (1998) concur, but go even further to explain CAI testing offers a unique opportunity for teachers to overcome the limitations imposed by traditional instructional delivery systems such as lectures, textbooks, A-V films or audio tapes that limit the student's ability to interact with the instruction. Such limitations mean teachers must write most of their learning objectives at the memory level, instead of at the performance level which is actually where higher educational testing should take place. As a result, many students are able to verbalize knowledge about things but lack confidence in their ability to perform the cognitive skills of their professions (Gibbons \& Fairweather). In nursing, several educators describe the advantages of computer adaptive testing (Bersky \& Krawczak, 1995) and virtual reality simulation software that asks students to demonstrate clinical decision-making skills at the performance level over traditional testing formats such as essay or multiple-choice questions (Lyons \& Milton, 1999; Thede, 1999; White, 1995).

The next two postinstruction strategies were included in the questionnaire because, according to the Bates (1999), many teachers fail to understand the implications of the choice they make when deciding whether to assign a CAI program as an "optional" or a "required" assignment and the impact the decision has on student learning (Kemp et al., 2001). Most who assign a CAI program as an "optional" assignment make the decision because they want to make the course more accessible to off-campus and working students. Then, motivated again by a desire to be fair, they do not include the information covered solely by the CAI assignment as 
part of the course assessment process. The problem is that the research convincingly shows that students who are not required to complete a CAI assignment and are not tested on the information seldom do the assignment. As a result, they simply do not learn the content or skills taught by the CAI and an expensive and potentially valuable learning tool ends up being a liability rather than an asset to the course (Bates).

The postinstruction integration strategies of "conduct a summative evaluation of student learning to test the effectiveness of the CAI in meeting the course objectives" and "provide alternative methods of restudying if results of tests indicate students failed to meet objectives using the CAI program" are both primarily implemented by teachers to increase student learning (Kemp et al., 2001). The strategies fall under the final two stages of instructional design theory of "evaluation" and "revision". The purpose of the evaluation is to provide decision-makers with information about revisions that need to be made. The utility of an educational evaluation is enhanced by immediacy, clarity, regularity, accessibility, and by maintaining a future orientation (Gustafson \& Powell, 1991).

In the case of the strategy "provide alternative methods of restudying if results of tests indicate students failed to meet the objectives using the CAI program," the evaluation method is typically a test score or some other assessment of the learners' ability. The decision-maker is the teacher who may, or may not, decide to provide students with alternative methods of restudying the material. The typical response in a higher education setting is for teachers to take more comprehensive steps if the entire class failed to meet the course objectives using a CAI program than if only one or two students did poorly. Since learners who spend additional time studying usually learn more regardless of which resource they use, the teacher may select from a number 
of different options (e.g., lecture, private tutoring, self-directed study, or a remedial exercise) to help the students improve their knowledge level. Some may decide to do nothing and leave the responsibility for remediation with the students. For instance, Goodman and Blake (1996) noted faculty at their school conducted the usual review of graded tests and engaged students in an inclass discussion but only if class performance data indicated $25 \%$ or more of the students missed a question.

The two common approaches to evaluate the software are formative and summative evaluations. A formative evaluation is ongoing throughout the instructional development process and is intended to help ensure the instructional unit achieves the stated goals (Flagg, 1990). One approach is to have students complete "mini- evaluations" after each CAI assignment to focus on the strengths and weaknesses, technical or delivery concerns, and content areas that need additional coverage. A summative evaluation is conducted upon completion of the course and is used to determine the overall effectiveness of the class or CAI learning experience. A summative evaluation usually focuses on student performance, relevancy of the unit, learners' attitudes toward the delivery methods, and the instructor's teaching style and effectiveness. Data are collected through quantitative and qualitative methods. Quantitative techniques rely on a breadth of responses and are patterned after experimental research through the collection and manipulation of statistically relevant quantities of data. The qualitative arm of the evaluation focuses on a depth of response and usually involves gathering more subjective data and anecdotal information from a relatively small, and possibly statistically insignificant, group of respondents (Flagg). Guba (1978) identified a number of qualitative methods for collecting evaluative data including open-ended questions, participant observation, non-participant 
observation, content analysis, interviews, and unobtrusive measures. Qualitative approaches may be of special value to the teacher evaluating the use of CAI for a single course because the diversity of students may defy statistically relevant stratification and analysis (Willis, 1993).

The need for revision should be anticipated by the teacher (Flagg, 1990). In fact, there will likely be more confidence in a course that has been significantly revised than in one that was considered flawless the first time through. Revision plans are typically a direct result of the evaluation process in-tandem with feedback from colleagues and content specialists but the best source of revision ideas may be the teacher's own reflection on the course strengths and weaknesses. Revisions may be minor, such as breaking a large instructional unit into more manageable components, increasing assignment feedback, or improving student-to-student interaction by switching from individualized to a small-group mode of instruction (Flagg).

Hammond et al. (1992) also noted that one of the less obvious, indirect ways teaching and learning are positively affected by CAI is a result of the amount of work teachers typically invest when integrating a CAI program into the course. The typical educator spends more time than usual concentrating and focusing on teaching and learning tasks as they work to adopt and integrate the technology into a course. While the extra work temporarily decreases the teacher's productivity because mastering and using the technology claims a large share of their discretionary time; first, to develop new approaches to teaching and then to fine-tune and improve them, but most faculty report they are intellectually challenged and find the adoption process rewarding, especially if the institution provides appropriate rewards. Far from a loss, many educators report the time spent tends to be worthwhile even if a particular technology experiment is not completely successful primarily because they are encouraged to pay closer 
attention to the fundamental principles of pedagogy and the overall quality of their instruction is improved as a result (Hammond et al.).

In summary, an abbreviated form of many well known instructional design theories (Alessi \& Trollip, 1985; Briggs, 1991; Dick and Carey, 1991; Gagne', 1985; Merrill, 1983; Reigeluth \& Schwartz, 1989) developed by Gustafson and Powell (1981) was used to explain the theoretical basis of the integration strategies that are primarily used by teachers to improve learning and decrease learning time. The first strategy of administering a "pretest" to ensure students are adequately prepared to learn the content of the CAI (Kemp et al., 2001) is done to ensure congruence between the students in the course and their level of preparation for the software program (Gustafson \& Powell). The use of poorly designed or the inappropriate application of software detracts from the course objectives, wastes students' time and may turn them against the media in the future (Gleydura et al., 1995). Conklin (1983) noted the effectiveness of CAI is often based on the teachers' selection of the most appropriate programs for the objectives of the course and the level of learner preparedness. Dick and Carey (1991) recommended teachers in higher education select software that is based on sound pedagogical principles and that uses instructional strategies that help learners achieve performance level objectives which should be introduced in early courses and continued throughout the curriculum. Khoiny (1995) reviewed the nursing literature and found that of the factors that impact the effectiveness of CAI, the quality of the software and the characteristics of the learners were important. There is a wide diversity in the quality of software for nursing education which means teachers need to carefully select affordable but high quality software that contains correct information, clear instructions, clearly stated objectives, correct sequencing, and appropriate and 
immediate feedback (Khoiny). The best programs for students in higher education begin at memory level but quickly advance to performance level objectives (Dick \& Carey, 1991). Other guidelines are to select programs that provide a variety of media and activities, positive, informative feedback and achievement measures, opportunities to practice and review and those that are both interesting and enjoyable (Goyne, McDonough \& Padgett, 2000). The two most important criteria, according to Bowell (1995), are programs that are interactive and engage the learner. Interactivity means application and practice and not just turning pages (Bowell). Other assessment criteria include determining if the software supports small-groupor wholeclasslearning and whether the time it takes to complete the instruction compares to the time it takes using other media. Another consideration is whether the school can afford to purchase enough copies of the program for the number of students in the course (Bowell). Cobb (2001) noted the need to match the level of difficulty of the software with the computer skills of the students. Cobb observed that students who lacked previous computer experience had difficulty accessing and proceeding through the lessons, became frustrated and had difficulty with the touch screen monitors (Cobb).

The next three integration strategies are intended to prepare students for the learning experience and have to do with explaining the CAI objectives, describing how to use the CAI, and explaining the value of learning the content of the CAI (Kemp et al., 2001). The three strategies are taken from some of the most basic tenants of instructional design theory and the idea that students do better when they know and understand beforehand exactly what they are to supposed to learn (Gustafson \& Powell, 1991). The strategies relate closely to Gagne's (1985) First Event of Instruction which is to gain the adult student's attention by verbally informing 
him $\backslash$ her of the objectives. The goal is to help the students' focus and sort out important from unimportant information so they save time and energy. Gustafson and Powell (1991) recommend the teacher inform the class of the objectives even when they are printed in the instruction. The next strategy of demonstrating or explaining how to use the CAI software program beforehand is also done to prepare learners for what to expect and prevent their being distracted by fears or concerns over how to use or navigate through the software program (Khoiny, 1995; Perciful \& Nester, 1996). Brudenell and Carpenter (1990) suggested that a lack of orientation to computers and software are possible reasons so many students have negative attitudes toward CAI. MacKeller and Elliott (1999) noted teachers pay too little attention to the needs of students for adequate training in how to use both computers and the CAI programs and often fail to provide users with the technical and instructional help they need.

The fourth strategy is to motivate students by explaining the value of learning the content of the CAI. It is also intended to help students understand why the content is important, how it relates to the rest of the course, and how it will be of value to them in the future. The fifth integration strategy of illustrating the relationship between topics in order to integrate the CAI with the rest of the course (Kemp et al., 2001) is similar to Gagne's (1985) Third Event of Instruction which is to present a stimulating recall of prerequisite knowledge before teaching new content. It is based on the assumption that all learning builds on what is already known which is especially true of complex, higher level thinking skills. Gagne' stressed the importance of learners having relevant prerequisites in their minds in order to integrate them with new knowledge but noted there are times it will mean students need to take some action with the knowledge in order to bring it into active memory. Lyons and Milton (1999) described an 
excellent example of how the faculty of a nurse midwifery program used a virtual reality computer simulation program to provide students with immediate practice with the prerequisite knowledge gained from the theory component of the same course. Thede et al.(1994) found that students believed teachers at their school should have done more to integrate the CAI programs they assigned into the course. Many thought a simple explanation from the teacher would have helped prepare and motive them. On the positive side, students said completing the CAI program helped prepare them for the class and as a result, their classroom participation and learning increased. Thede et al. recommend nurse educators take seriously the change in role from information provider to facilitator of the instructional process by providing support before, during and after CAI instruction.

The decision the teacher makes about whether to use an individualized, small-groupor a whole-class mode of learning has been shown to impact both learning and student's time to learn. There are many variables the teacher needs to take into consideration when making the decision such as the objectives of the course, the computer resources, the facilities, and the expertise of both the teacher and the students in the class, to name just a few. Gagne's (1985) Fourth Event of Instruction entails presenting facts, knowledge or skills in a context or background. The context or background may be working alone, in a small-group or in a classroom setting where the teacher and students interact with the CAI program (Wells \& Kick, 1996). No matter which mode the teacher selects, the goal, according to the model, is the help the learner focus attention on the relevant parts of instruction. This can be accomplished by the teacher (in a whole-class presentation) or the CAI program (in individualized or small-group instruction) by attention directing techniques or instructions, through learning activities or by 
modeling correct performance (Gagne'). Gagne's (1985) Fifth Event of Instruction is to provide the learner with an opportunity to practice to see what they do and do not know. The emphasis at this point is on learning and not on testing and the higher the level of learner interactivity with the instruction the better. According to Wittrock (1991), the best learning happens when the learner is mentally active in the learning process. Epper (2001) noted "active" learning implies students are doing or are directly engaged with the instruction.

Some of the advantages of an individualized mode of instruction are the ability to adapt instruction to each student's needs and provide one-to-one- tutorial quality instruction (Johnson \& Johnson, 1985). Assigning students to work alone makes it easier and more convenient for learners to access the instruction and save valuable time (Bates, 1999). Many CAI software applications are simply new applications of old methods that have been made more accessible. For instance, CD-ROMs and DVDs are being used to replace text and reference books and are able to save students' time by providing them with search capabilities. They also provide quick access to graphics, animations, sound and video (Fisher, 1997) so that students are able to watch and hear a multimedia presentation of a procedure performed by an expert rather reading about the procedure and trying to imagine how it is done (Gleydura, Michelman, \& Wilson, 1995).

The reduction of learning time depends on the way the instruction is designed. More learning can be accomplished in a given amount of instructional time if the developer has arranged things to make that happen (Gibbons \& Fairweather, 1998). Dick and Carey (1994) noted the best programs contain a sequence of instructional strategies that can be selected, applied, assessed and discarded until one is found to which the student responds. Some programs use artificial intelligence and multimedia to dynamically adjust instructional strategies 
to the student's responses (Gibbons, Fairweather \& O'Neal, 1993). An observant support system that is capable of monitoring, learning, coaching, advising, judging, providing feedback and assisting with the selection of the most beneficial learning paths increases learning and reduces the time it takes (Gibbons, Fairweather, \& O'Neal). Some of the most often cited advantages for students working independently with a CAI program include the following: the ability to set their own learning pace and tailor the lesson (Smith, Jones \&Waugh, 1986); the ability to adapt to different academic backgrounds (Conklin, 1983); and to adjust to the learners' interest, rate of learning, and knowledge-base (Levin, 1983). Others have noted improved motivation and enthusiasm for education, the development of critical thinking, and problem-solving skills and a better recall of information (Charp, 2001). The convenience of learning any place, any time (Scanlan, 1986) makes CAI an attractive medium for adult learners (Knowles, 1984; Reif, Roulis, \& Huber, 1995). Adults also like the fact that computers alleviate the need for memorization and hunting for information and allow them to plan their own learning, control the pace, decide when content is presented and the style of the presentation. Roblyer's (1988) review of the research that compared the effectiveness of CAI across age groups found the college and adult-learner populations did better than the other age groups. Lerner, Cohen and Brown (1990) also noted the self-paced format and unlimited practice feature of individualized CAI has the potential to provide instruction for an increasingly diverse student population in higher education.

Substantial reductions in learning time for individualized instruction were reported in several studies (Bitzer \& Boudreaux, 1969; Kulik, Bangert, \& Williams, 1983; Kulik, Kulik, \& Cohen, 1980; Larson, 1982). Tilson (1986) found students who used CAI spent 50\% less time 
than those who attended traditional classroom instruction. CAI been shown to reduce the amount of time it takes for individuals to learn (Huckabay et al., 1979) and reduce by one-third (Bowell, 1995) to two-thirds the time needed for a conventional course (Kulik \& Kulik, 1991; Clark, 1991; Reynolds \& Pontius, 1986). Even early studies based on programs that were less sophisticated text-based CAI programs showed substantial time reductions. Estimates vary from small reductions to one early study by Bitzer and Bordreaux (1969) that reported the CAI group learned equally well but cut their learning time from 66.5 to 23.5 hours. Hmelo's (1990) review of the health professions literature showed the only significant finding in favor of CAI was that it decreased students' learning time (Hmelo). Different authors attribute the time savings to different reasons. For instance, Bitzer and Bordreaux (1967) believed it was due to the learners' ability to pick and chose the content that is needed and skip portions that are not. Several researchers believed it is due to the better organization of the instruction (Bunderson et al., 1984; Clark, 1983; Kulik \& Kulik, 1991; Kulik, Kulik \& Cohen, 1980) and others to more individualized interaction and feedback (Bates, 1995a).

Several studies that used an individualized mode of instruction were shown to increase learning (Neafsey, 1997) and decrease students learning time were discussed. Bareford (2001) reported on one particularly innovative way an inexpensive commercially available computer simulation game was used to increase learning while dramatically reducing the amount of time students' normally spent on a community health assessment from weeks to hours. In another, Vincent (1984) compared individualized CAI with lecture-discussion and found no significant differences in student performance on the initial post-test score but the delayed post-test showed 
the computer learners' scores were significantly better despite the fact that the computer learners spent $18 \%$ less time on the instruction.

A small-group mode of CAI has been shown to offer both advantages and disadvantages in terms of student learning and students' time to learn. Working in small-groups means students interact with one another to accomplish shared goals while ensuring everyone masters the material (Johnson \& Johnson, 1985). Teachers primarily assign small-group work to teach collaboration and negotiation skills and to prepare students to work as members of a team. Other reasons might be to save time, to accommodate shortages in hardware or software, to provide peer assistance for academically weaker students, or to prevent novice computer users from being left behind (Cobb, 1999). Teachers need to select software that supports small or large group instruction when course objectives call for teaching collaborative decision-making (Belfry \& Winne, 1988).

The limited research on the effectiveness of group instruction with computers (Calderone, 1994; Webb, 1985; Weiss \& Jarvis, 1986-87) indicates the social interaction that occurs between students is how learning (Bejarano, 1987) and the development of problem solving skills occurs (Wizer, 1987). Two ingredients necessary for effective small-group learning are the teacher's guidance and placing the emphasis on peer interaction and dialogue (Bouton \& Garth, 1983). Miller (1990) promoted student interaction by requiring a group consensus before groups of two or three law students were permitted to put the answer into the computer. Castner (1992) found dyads of nursing students working at computers did more exploring than students who worked alone but the types of strategies they used were the same and both groups accomplished the course objectives. Watson (1966) identified many factors that 
facilitated group interactions which include the following: socialization, members' contributions; the presence of high ability and highly motivated members in each group to provide insight to others; rectification of individual blind spots; provision of security in risk-taking; and the canceling of chance error. Impeding factors included the following: lack of unity; frustration over interference and criticism; rambling talk by some; leaving the tasks for others; lack of motivation; opposing goals; and a failure to communicate (Watson). Simpson (1986) noted a significant drawback to group learning was the unequal contribution of group members which could foster resentment and undermine learning. Slavin (1983a) identified the problem as "the diffusion of responsibility" and noted it eventually led to diminished performance by all members. In a follow up study, Slavin (1983b) successfully addressed the need for individual accountability by stipulating beforehand that group success would be determined by a sum of the members' learning performance.

Additional suggestions and problems encountered when using a small-group mode of CAI were discussed. Khoiny (1995) suggested stretching scarce computer resources (computers and software) by dividing a large group of students into smaller groups and assigning scheduled times. Perciful and Nester (1996) noted small groups had problems because some students finished much more quickly than others which interfered with a post-simulation group discussion. They recommended assigning students to work individually and then schedule a post-simulation group discussion at a later time (Perciful \& Nester). Koch, Rankin and Stewart (1990) found students in their study preferred a small-groupover an individualized mode.

Baird and Koballa (1988) looked at the effect of group size and type of computer program on undergraduate students' perception of success at acquiring hypothesizing skills. The 
students worked individually or in groups of 3 to complete a simulation or a textual CAI exercise. Students who worked in groups on simulations had the highest mean scores of perceived success and students working alone on textual exercises had the lowest mean scores. Students who worked in groups also rated the software higher than individuals. They concluded the student's perceptions of success and attitude toward the instructional program may be influenced by both the type of CAI program and the mode of instruction (Baird \& Koballa).

There is conflicting evidence concerning the influence of factors such as gender, lesson content, and learner performance on the effectiveness of a small-group mode. Cobb (1999) compared homogeneous average achievement, homogeneous high achievement and heterogeneous mixed achievement groups of between 3 to 5 students and found no difference in achievement on immediate post-test scores. The group of homogeneous high achievement students scored significantly higher on a post-test given two weeks later. A qualitative analysis revealed no significant differences among the groups in their interaction frequencies or cooperativeness (Cobb). Noell and Carnine (1989) looked at the instructional effectiveness and efficiency of individualized and small groups using IVDI. The results of an 18 short-answer test revealed no significant difference in the achievement of individuals and groups of learners. However, the learning time for a group of 5 students was 40 minutes and for an individual was 30 minutes which meant 5 individuals would require 150 minutes to complete the assignment. The group mode was much more time-efficient without a loss in achievement (Noell \& Carnie). In another, Park (1993) assigned students to one of 4 cells based on learning group (cooperative group or individuals) and ability group (high or low ability). All participants spent a minimum of one hour a week working on the CAI assignment after attending the same lecture. The results 
showed both high and low ability students in cooperative groups improved in their performances more than those who worked alone, and yet, the attitudes toward the instruction were the same (Park). Whyte (1990) looked at the effects of learning styles (field independent, field dependent or mixed) on the way the groups were paired for small-group CAI learning and found no significant differences between those who worked individually or in pairs. However, the way subjects were paired made a significant difference in individual achievement test scores. Groups of either two field independent students or mixed groups of one field independent and one field dependent student significantly outperformed groups made up of two field dependents. Field dependents enjoyed working in pairs more than the others but the optimal pairing included at least one field independent student (Whyte).

Whole-class group or collaborative learning occurs through active participation by teachers and students (Hiltz, 1990; Whipple, 1987). Again, knowledge is thought to emerge through the classroom interactions rather than through the delivery of subject content by the teacher (Simpson, 1986). Observations of students' activities during computer use showed more social interaction when attention is directed at attaining educational goals. Just as with smallgroup learning, teachers were needed to facilitate and ensure group productivity (Simpson). Kilmon (1996) used whole-class CAI by first assigning students to complete the CAI assignment outside the classroom and then, used the same programs to stimulate whole-class discussion about the public health implications, ethical issues, or other relevant themes. Teachers also used case studies to guide students in learning various nursing skills (Kilmon). Goodman and Blake (1996) described using a series of CAI multimedia programs for whole-class teaching and reported the strategy turned a traditional passive classroom into an interactive environment 
where students were involved. Summative evaluations of the project revealed students met the class objectives of improved mastery of content and decision-making

The integration strategies of "providing technical and instructional support personnel to assist students" are primarily cost issues but have also been shown to impact both student learning and time to learn. Most teachers identified costs as their major concern when using CAI but said their second biggest concerns were the inevitable technical problems and how disruptive they might be to the course in terms of time lost (Cambre \& Castner, 1993). Bates (1995a) pointed out access rather than effectiveness is the most important criterion teachers need to consider when selecting an instructional delivery system. Access includes more that just purchasing enough computers. In order for students to learn and not waste valuable time, teachers need to ensure they have access to computers and software programs, that they be oriented to the computers and each new program, and are provided with adequate technical support (Khoiny, 1995). Access has become even more important as more students in higher education are becoming part-time adult students who need to be able to access CAI from offcampus sites. Even when students purchase use their own computers, the teacher still needs to ensure they have access to technical and instructional support over the phone. Some of the factors that need to be taken into consideration when deciding how much and what types of support are needed include things like how flexible the software is for students with different educational backgrounds, incomes, ages and prior learning and computer experience. When trying to decide if technical support is enough or if students also need instructional support the teacher needs to consider all of the above factors as well as the user-friendliness of the particular 
CAI program, the complexity of the course content and the objectives of the course as well as the sophistication of the learners (Bates).

The importance of having adequately trained and well-equipped personnel available at the computer lab is a common theme in the literature. Problems students experienced included lost time traveling to computer labs and problems with unavailable computers and software (Lowdermilk \& Fischel, 1991). Weis and Guyton-Simmons (1998) observed students who lacked previous computer experience appeared unsure and hesitant and those who lacked typing skills became easily frustrated. Written instructions without an explanation or assistance did little to help but students indicated a thorough explanation beforehand may have helped but having someone available would have been best (Weis \& Guyton-Simmons). Several authors provided suggestions for the ways, under the right conditions, the need for technical and instructional support personnel might be eliminated. One was to have students purchase their own computers or have the school purchase mobile laptop computers and provide phone access to technical or instructional help (Khoiny, 1995). Shortages of software could be lessened by providing students with access to CAI programs over a campus-wide network, or the Internet (Khoiny). McGinty, Reed and Sautter (1987) noted networks and using programs designed to run from a central file server eliminates the need for individual copies of software and helps ensure the safety of the stored discs.

The need for an adequate orientation for students who lack computer experience was noted by several authors (Bates, 1999; Khoiny, 1995; Weis \& Guyton-Simmons, 1998) ). The importance of properly trained, knowledgeable personnel who were available, willing to answer questions and help students resolve problems were stressed (Khoiny). Thede et al.(1994) noted 
students reported long waiting lines to use a computer, programs that were not user-friendly and compatibility issues between software and hardware were barriers. The need for retraining or sensitivity training was recommended for insensitive technical and instructional support personnel (Thede et al.).

The integration strategy of "conducts a post-instruction discussion or critique to reinforce what students learned and correct students' misunderstandings" (Kemp et al., 2001) correlates closely with Gagne's (1985) Sixth Event of Instruction. The goal is to provide feedback about the correctness or incorrectness of the learner's performance, an explanation of the level of accuracy, why some answers are correct and others are not. CAI programs usually provide responses such as "correct" or "incorrect," or provide hints, explanations, or suggestions (Gagne'). The more informative the feedback the better the quality of the CAI program (Thede et al., 1994). The next post-instruction strategy relates to Gagne's Eighth Instructional Event and is "provide follow-up activities to ensure students use what they learned in the CAI program." The goal is to enhance retention and the transfer of knowledge or to ensure students are able to use the new skill or knowledge in a variety of applications or apply it in other settings and situations. Both strategies relate to Gagne's Ninth Event of Instruction which is to provide a review or summary. For the adult learner, a review or summary can be provided verbally or with follow-up learning activities (Gagne'). In the Thede et al.(1994) study, students indicated they liked informative and immediate feedback and found the quizzes at the end of every section extremely helpful. They did not like software that limited feedback to "correct" or "incorrect." but they thought feedback should have corrected their misconceptions. 
The two opposing integration strategies that deal with whether to make a CAI assignment "optional" or "required" were included in the questionnaire because, according to Bates (1999), most teachers make the wrong choice. Teachers fail to understand the long-term implications of the choice they make when they try to make the course more accessible to off-campus students (Bates). The topic is covered more fulling under the section on reducing course costs.

The final post-instruction integration strategies that describe "conducting a summative evaluation of student learning to test the effectiveness of the CAI in meeting the course objectives" and "provide alternative methods of restudying if results of tests indicate students failed to meet objectives using the CAI program" are both primarily implemented by teachers to improve student learning (Kemp et al., 2001). The strategies related closely to "evaluation" and “revision," stages of instructional design theory (Gustafson \& Powell, 1991). Educational evaluations are intended to provide decision-makers with information about revisions that are needed. Teachers typically make the decision about whether or not to "provide alternative methods of restudying if results of tests indicate students failed to meet the objectives using the CAI program" based on a number of variables. Some teachers may opt to re-teach the content another way, others may simply provide adult students with the page numbers of a textbook to read and still others may do nothing (Goodman \& Blake). .

A formative evaluation is an ongoing process to evaluate the software (Flagg, 1990) and a summative evaluation is conducted at the completion of the course and focuses on students' performance, relevancy of the unit, and learners' attitudes. Data are collected through quantitative and qualitative methods. Quantitative techniques include the collection and manipulation of statistical data and qualitative data usually involves gathering subjective data 
and anecdotal information from a relatively small-group of respondents (Flagg). Teachers should anticipate the need for revisions and will probably have more confidence in a course that has been revised. Revisions are often the result of evaluations, feedback or the teacher's own reflections on the course strengths and weaknesses. They may be minor or major and should be made according to priority (Willis, 1993). Hammond et al.(1992) noted the integration of CAI indirectly affects teaching and learning because the teachers typically spend more time than usual concentrating and focusing on teaching and learning tasks when they integrate the technology into a course. The productivity of the course temporarily decreases during the process but most faculty report they find the adoption process rewarding and the quality of education is improved as a result (Hammond et al.).

The integration strategies of "include the content of the CAI program on the course exam" and the other about "include scores earned on tests embedded in the CAI as part of the course grade" are usually implemented by teachers to either increase student learning or decrease the time the teacher spends on tests. While the strategies would seldom be used simultaneously, the rationale for both is the same, to motive students to do the assignment and to help them view the CAI assignment as an integral part of the course and deserving of the time and energy it takes to learn the content. The strategies are closely related to Gagne's Seventh Event of Instruction which is to assess the learners' performance by having them do a complete demonstration of learning outcomes without assistance, prompting, or guidance. According the model, if the course objective says "demonstrate" learners should be asked to perform the task and not to describe how or to answer questions about how something is done. Gibbons and Fairweather (1998) concur and note testing with some interactive CAI programs provides the teacher with a 
unique opportunity to write objectives and test at the performance level rather than the memory level. They blame the media used to deliver instruction for higher education for imposing limits that result in many students being able to verbalize knowledge about things but lack confidence in their ability to perform the cognitive skills of their professions (Gibbons \& Fairweather). In nursing, many educators describe the advantages of using simulation programs (Lyons \& Milton, 1999; Thede, 1999; White, 1995) and computer adaptive testing to have students demonstrate clinical decision-making skills at the performance level (Bersky \& Krawczak, 1995).

Next, the literature review will focus on integration strategies that are primarily used by teachers to reduce course costs.

\section{Strategies to Decrease Costs}

Dean (1989) noted one of the most sensitive and difficult ways to attempt to achieve economy in higher education is by making changes in the instructional process. However, the financial crisis in the higher education system of the United States has forced institutions to change the way they do almost everything and as a result, schools are increasingly turning to computerized instruction as one part of the solution (Dean). Different groups of professionals have different opinions about how CAI should be used to increase educational productivity. For instance, many teachers believe CAI is a good way to improve the quality of education but may actually be opposed to using it to increase student enrollments because they believe the quality of teaching will suffer. Many politicians, administrators, and business leaders equate technology with improved efficiency which, in their view, will be achieved by replacing labor and reducing educational costs. Tony Bates (1999), an expert and researcher in the area of educational technology costs, noted such individuals think the way they do simply because they fail to 
recognize the nature of the educational process. While labor costs can be reduced by applying technology, unless it is done skillfully, it can also lead to a large decline in the quality of student learning and a poorly prepared workforce (Bates). The goal in education should always be to improve cost-effectiveness and not just to reduce costs.

The usual method of measuring cost-effectiveness is to calculate the cost per student per hour of instruction (Bowell, 1998). Following that formula, the more frequently a film, or set of slides or series of overhead transparencies are used, the more the costs are distributed and the more cost-effective the technology becomes. However, because most CAI programs are so rich in information, the usual method of calculating the cost-effectiveness of CAI is to determine the cost per student multiplied by the number of times the instruction can be used without significant repetition. The alternative calculation method takes into account the number of times the same learners can use the program to gain new insights, extract new information or view the lessons from a different perspective. The most cost-effective programs can be used many times without repetition by many learners (Kemp et al., 2001). The costs should also be calculated over the number of years it will be used rather than for a single year or course (Bates, 1995b).

Massy and Wilger (1998) noted the areas of higher education that can profit most from CAI technology are subjects that have a high volume of students and a standardized curriculum that seldom need to be updated. Based on the principle of economies of scale, after a somewhat large front-end investment for hardware and software, the cost of use per incremental unit and per student with CAI is apt to be lower when the student body is large and when large amounts of information can be accessed at a low incremental cost using the same technology (Massy \& Wilger). 
Replace traditional instruction. The integration strategy of "using the CAI program to replace some other method of teaching that content" (Kemp et al., 2001) was included in the Integration of CAI Questionnaire as a cost consideration because the topic has generated so much discussion and controversy in the literature. Many authors report that CAI is used most often as a supplement or to enhance the primary method of teaching despite considerable research evidence that shows that supplemental use seldom improves learning outcomes enough. Bates (1995b) and Laurillard (1993), who have both published extensive literature on the topic of the costs of educational technologies, both noted that in order for CAI to be cost-effective it must be used in place of, and not in addition to, the traditional method of teaching. The rationale is that CAI is simply too costly to use as a supplement and there are many other and much less expensive ways to increase learning. Computer assisted-instruction should be reserved for those situations when the learning objectives cannot be met with a less expensive method or media (Bates, 1995b).

Required vs. Optional. The evidence is fairly conclusive that the way CAI is used may have more of an impact on the costs of the course than the fact that it is used (Bates, 1995b). While administrative level decisions such as the location of the school's computer lab, the numbers and types of computers the school purchases, and faculty promotion and tenure policies have a large impact on whether or not CAI technology is used optimally (Levin, 1986), but decisions made by individual teachers about how each CAI program is integrated into each course also impacts optimization (Massy \& Wilger, 1998). Bates (1995a) noted it is common practice for teachers to integrate a tutorial or simulation program into a course so that the content is taught by the CAI program and then again in the classroom. While the teacher should improve 
the conditions for student learning by adequately preparing students for a CAI learning experience, repeating the same content using different delivery systems simply has not been shown to improve student learning enough to justify using two such expensive delivery systems. The idea is to optimize the technology which means reorganizing the course so that the teaching that can best be accomplished by CAI moves away from fixed, scheduled group instruction to more flexible, individualized or group modes of learning and, based on the needs of the particular group of students, creating a balance (but not an overlap) between face-to-face and technology-based teaching (Bates, 1995a).

From a purely financial perspective, Massy and Wilger (1998) noted few extant technology applications save money. In other words, when CAI is integrated as an add-in or supplement to a course, the cost of the technology simply adds to the costs of the course. Similarly, personal aid applications such as word processor programs, data base or Internet software programs may save students' learning time and improve the quality of teaching and learning but traceable cost savings from such programs are rare. Most of the successful technology applications have been found to improve net productivity (benefits, adjusted for quality changes, divided by cost), but gross productivity (the number of output units divided by cost) generally declines. In other words, cost-effectiveness may be achieved but it is through the benefits afforded by the technology and not because the costs of the course are reduced (Massy \& Wilger).

The nursing education literature is mixed in the reports of different studies concerning whether or not CAI was primarily used as a supplement or as the primary mode of instructional delivery. For instance, Milkan and Aiken (1992) surveyed 512 undergraduate nursing programs 
and found that CAI use, at that time, was primarily for enrichment and remedial activities. Most programs were used as a supplement to classroom (72\%) and clinical learning (53\%) but few nurse educators used it to replace either classroom or clinical experiences (Milkan \& Aiken). In a meta-analysis of 11 different studies in nursing education, Belfry and Winne (1988) found CAI was used in eight of ten studies to replace traditional instruction. A later study by Hmelo (1990) that consisted of a review of the literature and covered a 20 year period sought to determine the effectiveness of the CAI software used for medical, nursing, pharmacy and other allied health professional education. Most of the software was intended for medical and nursing students and of those, $75 \%$ reported using CAI as a supplement to traditional instruction and only $23 \%$ used it as the primary method of instruction.

There is also disagreement in the literature about whether or not the use of CAI as a supplement improves learning enough to justify its use. In the Hmelo (1990) review (discussed above), the analysis revealed no significant difference between the supplemental CAI and the control groups on examination of performances. Likewise, a long list of early researchers found no difference in terms of student achievement on examinations when CAI was used as a supplement to traditional teaching methods (Conklin, 1983; Kirchhoff \& Holzemer, 1979; Klaassens, 1988; Larson, 1982). Based on the effect size of the studies in the Belfry and Winne (1988) meta-analysis (discussed above), CAI was more effective when it was used as a supplement to lecture or printed instruction. The effects for student achievement always indicated better results with CAI plus lecture than with lecture alone but when one outlying study by Conklin (1983) was removed, CAI had no measurable advantage over printed instruction. However, in two other studies by Boettcher, et. al (1981) and Belfry and Winne 
found the reason printed instruction was as effective as CAI when both methods covered the same content was because the full capabilities of the computer were not implemented.

There are many accounts in the literature of the ways educators have used CAI to decrease course costs. For instance, Engberg and White (1998) described a series of videoenhanced CAI simulations they developed to teach clinical decision-making skills to advanced practice nurses (APNs). The program allowed the students to query the computer using natural language in much the same way they would interview an actual patient and used interactive video to provide non-verbal clues of normal and abnormal physical findings. The program substantially reduced the course costs by using the simulations to replace the ongoing costs of live mock patients that had been used for the PBL courses. Several evaluations of the program were conducted including a research study to compare novice and expert students' clinical decision-making skills in which students were asked to evaluate the credibility of the simulations. The results indicated their responses where overwhelmingly positive. Another part of the research compared the students' knowledge of course content by comparing those taught by simulations with students taught by the traditional methods of instruction and there were no significant differences between the two groups (Engberg \& White).

A number of studies demonstrate how the cost-effectiveness of CAI was improved by optimizing its use. Merril and Barker (1996) described the development of a custom-designed virtual reality program to teach nursing students how to insert intravenous catheters. The project was the collaborative work of a group of six nursing teachers and a team of professional software developers. The project was initially funded by a private grant but the final costs were so high, the teachers needed to off-set some of the costs by sharing the program with other schools within 
the college that also taught their students IV skills (i.e., laboratory technicians, emergency medical personnel, and physicians' assistants). In another, Jefferies (1999) described a virtual reality program that his hospital used as part of an orientation program to teach nurses and various other hospital employees how to perform a 12-Lead ECG. Before the program, new employees attended a day long class that was taught up to 5 times a week by hospital in-service teachers. Once implemented, and despite its high developmental cost, the program proved to be cost-effective in that setting because it was used repetitively to free-up the in-service teachers' time. Goranson (1997) also compared the cost-effectiveness of CAI with the costs of traditional lecture for in-service training on standard safety precautions in a tertiary health care system. A random sample of $(\mathrm{N}=85)$ nurses were divided into two groups; one group completed the CAI and the other attended a lecture. A pretest posttest was used to measure learning differences and the cost of delivering both methods was calculated. The results showed that while both groups met the learning outcomes, the CAI was more cost-effective than lecture.

As mentioned previously, a common misconception among educators concerns the decision about whether or not to make a technology delivered assignment "optional" or “required" (Bates, 1999). Most teachers believe assigning a technology assignment as a required part of the course would limit the overall access to the course for working and off-campus students. However, once the assignment is designated as "optional" or "highly recommended," again, motivated by the desire to be fair, the teacher typically avoids delivering "essential" or "tested" material exclusively through the technology medium. Then when the information does not appear on the course test and students recognize there is no substantial penalty for failing to complete the assignment, most opt to spend their limited study time on assignments that count 
toward the grade. The end result is that an expensive and potentially valuable learning resource goes unused and becomes a detriment to the productivity of the course rather than an asset (Bates).

An interesting study that illustrates this point was conducted by Collis and Moonen (2001) who found almost an entire class of second year university students were willing to expend considerable effort completing boring, time-consuming annotations to ten research papers because the teacher announced at the beginning of the course that (depending upon the quality of the annotations) the assignment may have a positive impact on the students' final grade. In the same course, fewer than $5 \%$ of the students attended an alternative enhancement activity that consisted of a series of high quality, interesting videotapes that were shown one hour before the start of each lecture period. The only requirement of the second option was an investment of the students' time. The results showed that when students were explicitly made aware of some advantage for attending or participating in an enrichment activity, they invested large amounts of effort. The researchers concluded that educators were very much mistaken if they believed that just by adding a new media to a course they would be able to affect student learning because the way it was integrated had a significant impact on its success (Collis \& Moonen) .

In the Thede et al., (1994) study, graduate and undergraduate nursing students reported a major barrier to their use of CAI was they had so many demands on their time that they needed to prioritize and spend their study time wisely. All 12 subjects said they needed to know the time they spent was an efficient use of their study time. The number one criterion students used to determine "efficiency" was whether or not the information they learned would be included on 
the course test. Several mentioned that even if it was included on the test, they wondered if the penalty for not answering correctly would be substantial enough to warrant the time they spent on the assignment. One student noted from her past experience optional and supplemental assignments were usually not valuable, most were outdated or simply not pertinent (Thede et al.). McFarland (1993) noted that although nursing students rated their learning experience with CAI as very high they also expressed concerns about adding an additional activity to an already over-inflated curriculum. She recommended faculty use caution in assigning CAI as part of course assignments and be certain that what is assigned fulfills the course objectives and is not merely a distraction that might lead students to use their time unwisely (McFarland).

In another, Greipp (1988) described an attempt on the part of the faculty of one SON to integrate newly purchased CAI by designing a master plan for exactly how, when and where CAI would be used throughout the curriculum. Students were told the CAI was available and could be used on a voluntary basis to supplement traditional instruction. An evaluation conducted at the end of the first semester revealed students' use was less that $10 \%$, or 1.3 hrs per student. The following semester, the faculty designated CAI time as mandatory and required each student to complete 20-30 hours of instruction for each course. Students took a quiz at the completion of each CAI program that counted as $10 \%$ of the course grade and attendance records were kept on the time students spent in the lab. Class records indicated near $100 \%$ completion rates at the end of the second semester and students' rated the CAI learning experiences favorably (Greipp).

Arnold (1990) developed and tested a student CAI software evaluation form intended to capture students' input for part of the educational evaluation process. During the three-year 
evaluation period, she noted the way instructors assigned the software determined whether or not students completed the CAI assignments. Some instructors stated the assignment was required and monitored its use while others simply stated it was required and designated a point value that would be added to the course grade. The students who did the assignments were the ones enrolled in courses where the instructors followed up and followed through by checking before the end of the semester to make sure students did the assignment before the student passed the course or received the bonus points (Arnold).

Modes of Instruction. There are numerous cost considerations the teacher must keep in mind when deciding on which mode of learning to use when assigning a CAI program. One way the individualized or small-group modes of instruction are thought to improve educational productivity is that they make better use of the student's time and capacity for self-paced or peerassisted learning while, at the same time, freeing up more of the teacher's time to plan and manage the learning process and assist more students (Johnstone \& Maloney, 1998). By having small groups or the whole-classof students work from a single computer, teachers can improve the cost-effectiveness of the course by reducing both the teachers' and students' time to complete the assignment and reduce the number of computers and software programs needed for the course (Calderone, 1994; Fletcher, 1992; Noell \& Carnine, 1989). One the other hand, the need to purchase a projector or monitor that is large enough to permit viewing of the content by all the students in a classroom may increase the costs or even prohibit the use of CAI for wholeclasslearning for some schools that lack that the resource (Bates, 1999).

Sutter and Reid (1969) conducted a study of randomly assigned college-level students $(\mathrm{N}=100)$ taking a CAI problem-solving course. Instruction was presented to a control group ( $\mathrm{n}$ 
$=18$ ) with traditional lecture discussion and two experimental groups who used CAI as individuals $(n=40)$ or in pairs $(n=42)$. There were no significant differences between the three groups in achievement or attitude but pairing permitted twice as many students to be taught with the same number of computers which resulted in a substantial cost savings.

The mode of instruction may be chosen based on the teacher's or learners' preferences but still result in cost-savings. For instance, Straker (1988) described a study $(\mathrm{N}=18)$ in which a single mathematics videodisc was used for classroom instruction. The teaching strategies included direct instruction, student interaction, and regularly administered tests. The mode of learning permitted teacher-pacing of the instruction so that a certain level of mastery had to be achieved by the group on the tests before the teacher progressed to the next section of the lesson. Student's results on achievement tests revealed the large-group mode of instruction resulted in a high level of student interaction and it was a cost-efficient and effective method of teaching (Straker).

Technical and instructional support. Depending on how the school budgets for computer lab resources, another decision the teacher may need to make is whether or not to include the costs of technical and instructional support personnel in the course budget. As noted previously, the costs of delivering CAI services go far beyond the costs of purchasing hardware and software (Levin, 1983). Schools also need to provide secure facilities to house the computers, knowledgeable personnel, instructional materials, equipment such as printers, storage devices, and networks as well as maintenance personnel and other support services. In fact, the major expenses for CAI are for the ongoing operation of the computer lab such as the salaries of 
technical and instructional support personnel which when spread out over the life of a computer are as much as five times as much as the costs of the hardware (Levin).

The wide discrepancy that existed between what schools spent for CAI services narrowed somewhat in the 1980s and 1990s when schools feared being left behind and invested heavily in computers, networks and computer laboratories (Massy \& Wilger, 1998). More recently, many schools have gone in the opposite direction and have cut their CAI expenses to a bare minimum by opting to have students purchase their own computers and are providing access the schools' CAI software over a campus-wide network (Khoiny, 1995; Young, 1997). As a result, the trend has moved back again toward an increasing disparity between what schools spend for CAI services. An indirect outcome of the trend is that there are almost no research studies being conducted that focus on the costs of providing computer services to students. The reason is that the findings of such studies have little or no meaning from one institution to the next (Levin, 1986). The only costs that have remained somewhat consistent and can be compared across institutions are the costs of providing technical and instructional support personnel. Based on that reasoning, the strategies of provide for "technical" and "instructional" support personnel were included in the questionnaire and questions about the costs for maintaining a computer laboratory were not. Also based on that same reasoning, it is expected that teachers who are employed by schools where the teacher calculates his or her own course budget and are therefore aware of how expensive the support serves are will report the integration strategies significantly increase the costs of the course (Dean, 1989). It is also noted, according to Bates (1999), that few educators are aware of the course budgets or the costs of providing such services since those types of concerns typically fall under the responsibility of school administrators. 
In summary, the financial crisis in higher education has forced institutions to change the way they teach and as a result, most schools now used computerized instruction in order to economize (Dean, 1989). Different groups of professionals view the use of computers to reduce costs differently. Teachers are often in favor of using it to improve learning but oppose using it to increase class sizes. Business people view it as a way to reduce labor costs, but Bates (1999) pointed out the goal in education should always be to improve cost-effectiveness and not just to reduce costs. Cost-effectiveness is usually calculated as cost per student per hour of instruction which means the more often a unit of instruction is used the more cost-effective it is (Bowell, 1995). Because CAI is such a complex medium, cost-effectiveness is calculated by looking at the number of times it can be used without significant repetition. The most cost-effective CAI programs can be used many times with different sets of learners (Kemp et al., 2001) and over several years (Bates, 1995b). Massy and Wilger (1998) suggest those areas of higher education that can profit most from CAI are subjects that have a high volume of students and a standardized curriculum. The cost per incremental unit is lower when large amounts of information can be accessed with the same technology (Massy \& Wilger).

The integration strategy of "using the CAI in place of some other method of teaching that content" (Kemp et al., 2001) has generated considerable controversy in the literature. Many researchers note CAI is used most often to supplement traditional instruction even though there is evidence that suggests it probably doesn't improve learning outcomes enough. Bates (1999) and Laurillard (1993) warned the costs associated with CAI are so high, the only way it can be cost-effective is by using it to replace traditional teaching and when its use is optimized. In most 
instances, CAI should be reserved for times when the learning objectives cannot be met with a less expensive media.

The way CAI is integrated into the course has more of an impact on the costs of the course than the fact that it is used (Bates, 1995b). For the most part, administrative level decisions impact whether or not the technology is used optimally but decisions made by individual teachers also have an impact on optimization. Bates recommended reorganizing courses so that the teaching that can best be done by CAI moves away from fixed, scheduled group instruction to individualized or small-groupmodes of learning. Courses should have a balance of face-to-face instruction and technology-based teaching based on the students' needs (Bates).

Massy and Wilger (1998) noted few extant technology applications save money. When CAI is integrated as an add-in or supplement, the costs of the technology simply add to the costs of the course. Likewise, personal aid computer applications, such as word processors, database programs, and Internet software programs save students' time and improve the quality of education but they also do not save money. The cost-effectiveness that is achieved in both types of software applications is through the added benefits but not by reducing costs (Massy \& Wilger).

There are mixed reports in the literature concerning whether or not CAI is used most often as a supplement or as the primary means of instruction. Milkan and Aiken (1992) found most CAI was used as a supplement to classroom and clinical instruction but seldom to replace either one. Belfry and Winne (1988) found CAI was used in 8 of 10 studies to replace traditional 
Integration of CAI 101

instruction but in Hmelo's (1990) review, 75\% reported using CAI as a supplement and only $23 \%$ used it as the primary method of instruction.

There are also conflicting reports about the effectiveness of CAI when it was used as a supplement to a traditional mode of instruction. In Hmelo's (1990) study, the results showed when CAI was used as a supplement it did not make a significant difference in terms of learning performance. Several other older studies conducted in the area of nursing education also did not find supplemental use made a difference in learning outcomes (Conklin, 1983; Kirchhoff \& Holzemer, 1979; Klaassens, 1988; Larson, 1982). The meta-analysis study conducted by Belfry and Winne (1988) appeared, at first, to show supplemental use made a difference until one outlying study by Conklin (1983) was removed and then the results were no difference. A possible alternative explanation for the insignificant results might have been that in one of the studies (Boettcher et al., 1981) the CAI and paper forms of instruction were exactly the same and the computers were not used optimally.

There are several accounts in the literature that show ways educators have used CAI to decrease course costs. For instance, Engberg and White (1998) described using a series of CAI simulations to replace the traditional PBL method of teaching. The results showed the CAI was as effective and much less costly than the mock (actor) patients they had previously used in their curriculum. Several authors describe the "optimization" of CAI as the way to improve its costeffectiveness. Merril and Barker (1996) described the way the faculty of their school optimized the use of an expensive custom-designed VR program by sharing the program with several other schools in their college that also taught IV therapy skills. Jeffries (1999) noted a expensive VR program designed to teach hospital employees how to perform a 12-lead ECG was shown to be 
cost-effective in that setting because it was used to replace an in-service educator who would have otherwise needed to teach the course almost daily. In a tertiary health care setting, Goranson (1997) compared the learning effectiveness and cost of delivery of a CAI program with a lecture group and found both groups met the objectives but the CAI was more costeffective.

The decision about which mode of instruction to use requires the teacher keep in mind numerous cost considerations. By assigning students to work in small groups, the teacher can improve the productivity of the course by reducing both the teacher's and students' time to complete the assignment and lower the costs by reducing the number of computers needed for the course (Calderon, 1994; Noell \& Carnine, 1989). On the other hand, the need to purchase a computer projector or a large monitor to permit adequate viewing of the content by the entire class may increase the costs or even prohibit the use of CAI for whole-class learning for some schools (Bates, 1999). A small-group mode or the pairing of students at a single computer has been shown to reduce costs without a loss in achievement. Sutter and Reid (1969) found no difference between individuals and pairs working with CAI in achievement or students' attitudes. Teachers' or learners' preferences may be the reason for selecting a mode of instruction but may still reduce course costs. For instance, Straker (1988) used a single copy of a mathematics videodisc for classroom instruction and found it yielded a high degree of student interaction and was an effective method of teaching.

Depending on how the school calculates their budget for computer lab resources, some teachers may need to include the costs of technical and instructional support personnel in the course budget while others will not. Of the many expenses that go into providing students with 
CAI services, the most costly are for the ongoing salaries for technical and instructional support personnel (Levin, 1983). Many schools are cutting their costs by having students' purchase laptop computers so they can access the school's software over a campus wide network (Massy \& Wilger, 1998). This has resulted in a widening of the gap between what schools spend for computer instruction and a dramatic decline in the number of research studies that examine the cost-effectiveness of CAI. The only expenses that have remained somewhat constant and are comparable across schools are the costs of providing support personnel. It is expected that teachers employed by schools that require they include the costs of these services in the course budget will report providing these services is a significant cost factor (Bates, 1999).

The decision about whether to make a CAI assignment "optional" or "required" has generated considerable debate in the literature. When the teacher designates a CAI assignment as "required," the course becomes less accessible to off-campus and working students but by designating it as "optional," without realizing it, the teacher actually lowers the productivity of the course by reducing students' incentive to use the software. Several studies demonstrated this principle but one of the best was conducted by Collis and Moonen (2001) who offered students the option of completing boring, time-consuming annotations to research reports for extra credit or attend interesting, high quality videos for a hour before the lecture but for no grade advantage. The results showed students went to great lengths to complete the extra assignments but only if they were explicitly made aware that their participation may be of some advantage to their course grade. In the Thede et al. (1994) study, graduate and undergraduate nursing students reported a lack of time was a major barrier to their using CAI. Most needed to know they would receive credit for completing the assignment that would be reflected in the course grade. McFarland 
(1993) advised nursing faculty to be cautious of adding more to an already over-inflated nursing curriculum. Greipp (1988) described the need to make CAI assignments mandatory before students at their school of nursing completed them. Arnold (1990) also recommended making the use of CAI programs required in order to motivate students to use the software programs.

\section{Strategies to Decrease the Teachers' Time}

As previously discussed, one of the primary reasons teachers give for not adopting CAI is that they lack the time (Lewis \& Watson, 1997). Even teachers with a considerable amount of experience using computers report the time it takes to plan, prepare, integrate, evaluate and make adjustments to the course is the major barrier to its use. And, the research has shown that most teachers' productivity levels decline for as long as two to three years after they begin the adoption process (Cartwright, 1994). However, the same teachers report there are major advantages to using CAI and, once it is fully integrated, considerable time savings can be realized (Bailey, 1994; Hammond et al., 1992). While the teacher needs to plan some in-class or clinical time during each course to provide explanations, for review and to help students integrate the CAI lesson with the rest of the course, the amount of time the teacher spends implementing those strategies is generally less than if the CAI were not used. The most important reason teachers have for using CAI is so they can change their role from a "disseminator of knowledge" to a different, more effective, but relatively less time-consuming-role as a "facilitator of learning" (Bailey).

Replace traditional instruction. As mentioned previously, the costs of providing a nursing education are second only to a medical education and both are expensive because they are clinically-based professions (Ronald, 1991). The bottom line is that the schools must employ 
large numbers of teachers to teach clinical courses which means their budgets are usually much larger than other schools in higher education with equal or larger student enrollments. As a result, nursing is in a somewhat unique position because whenever CAI can be used to replace some portion of the teacher's time in the clinical area, the results are usually substantial costsavings (Perciful \& Nester, 1996). Therefore, even though CAI is less cost-effective with both large and small numbers of students than any of the other instructional delivery systems available, with careful planning and skillful course design, it is still possible to use CAI to increase the costeffectiveness of a course (Bates, 1999).

Several studies have demonstrated the use of CAI for decreasing the teachers' time. For instance, Perciful and Nester (1996) found CAI freed up an 8-hour day each rotation for the teacher while it increased student learning. Umlauf (1990), a hospital in-service staff educator, reported the use of a combination CAI virtual reality and IVDI to re-certify a group of hospital staff nurses and other employees in Cardio-Pulmonary Resuscitation (CPR) permitted learners to work independently with the CAI instruction but still demonstrate the life-saving procedure on a simulator-type, "Annie" to the levels specified by the American Heart Association (AHA). The CAI instruction was shown to be extremely cost-effective because it permitted around-the-clock accessibility to training during the employees' down time and eliminated for costs of an in-service educator who would have had to teach and administer the re-certification test (Umlauf). Jairath and Fitch (1994) used a research protocol development CAI program to guide staff nurses in developing nursing research proposals. Nurses who used the program were able to develop the proposals in much less time and the program permitted a substantial reduction in the in-service educator's time and editorial efforts (Jairath \& Fitch). 
While the potential for cost-savings in nursing education is greatest when CAI is used to replace clinical hours, the same principle holds true for a traditional classroom or clinical laboratory setting where the teacher-to-student ratio is typically much higher (Cambre \& Castner, 1993). The research shows that using the two most costly delivery systems, teacher-directed instruction and CAI, to teach the same content not only duplicates the unit costs of the instruction but it also lowers the productivity of the course even further by nearly doubling the time the teacher and the students spend on that content (Bates, 1999). The most productive approach, in terms of reducing the time the teacher spends teaching, is to designated a CAI assignment as a "required" part of the course. As mentioned previously, the most effective way to ensure students do the assignment is to advise them beforehand that the content of the CAI will either be included as part of the course grade through testing or that the assignment must be completed before the student passes the course (Arnold, 1990).

Modes of Instruction. As with so many other issues, different authors have different opinions about whether individual, small group, or a whole-class mode of instruction is more efficient for saving the teachers' time. For instance, Johnstone and Maloney (1998) believe educators should assign students to work outside the classroom to make better use of the student's time and capacity for self-paced or peer-assisted learning while freeing more of the teacher's time to plan and manage the learning process and assist more students.

While the research available on saving teachers' time through the use of CAI for wholeclass instruction is limited, Goodman and Blake (1996) conducted a summative evaluation of a faculty software development project and found the teachers' time was reduced when students interacted with peers at the computer and when they worked with the teacher on whole-class 
simulations. The study did not compare which mode saved more of the teachers' time. Yanok (1988) assessed whole-class presentation time and concluded it was time-efficient for the teachers and students and was effective in terms of learning outcomes. Kemp and Dayton (1985) noted large-group CAI instruction is generally considered less flexible in terms of the pace of learning and preference of study methods by individual learners but it does serve other needs. The primary benefit is efficiency in terms of teachers' time because it permits the content to be presented to large numbers of students at one time and avoids the need for repetition. He recommended using a whole-class mode of instruction for the following reasons: 1) to introduce new topics and orient students to a unit of study; 2) provide motivation through the use of alternative teaching strategies such as multimedia presentations; 3) illustrate relationships between topics; 4) present new content that is too recent to have been incorporated in a self-paced program; and 5) to provide enrichment experiences (Kemp \& Dayton).

While many teachers oppose the idea, course productivity can also be improved by increasing the number of students taught in a course while holding steady or reducing the time the teacher spends on the course. Walvoord and Pool (1998) provided an excellent example of the way one teacher redesigned a large freshmen level college course to improve its costeffectiveness while maintaining student learning, levels of satisfaction and student retention rates constant. The redesign incorporated several innovative teaching strategies but the primary one was changing the students" "first exposure" (to new information, concepts, or procedures) from a classroom lecture to an out-of-class independent assignment. Students were required to watch a series of instructional films and complete a written assignment prior to attending an abbreviated teacher-led discussion of the lesson. Since students had already processed the information before 
attending the class, they were expected to synthesize, analyze, and use the new information to solve problems and apply it to actual situations in response to teacher or peer questions during the in-class discussion period. The teacher skimmed over the written assignments and gave students credit for "good faith efforts," rather than actually spending time grading the assignments. Course grades were based on written examinations, written assignments and participation in class discussions. The results of the course analysis showed student enrollment was increased by $40 \%$ but the teacher actually spent $20 \%$ less time on the course. The technology was integrated so that it increased productivity but the follow-up assignments and in-class teacher led discussions were equally important for ensuring the high levels of student learning, satisfaction and retention (Walvoord \& Pool). The A-V films could easily be replaced with any technology, such as a CAI program, for the "first exposure" learning experience.

Testing strategies. The integration strategies of "include the content or skills learned from the CAI program on the course exams" and "include scores earned on tests embedded in the CAI as part of the course grade" (Kemp et al., 2001) are usually implemented by teachers to motivate students to do the assignment and learn the content. Another equally valid reason in terms of productivity, is to reduce the time the teacher and the students spend on testing. Most CAI programs include embedded tests that may either be optional, so that the learner can skip them if they prefer or required, so that students must earn a passing score before they are permitted to progress to the next instructional unit. Some programs are designed to not only administer the test but to record the student's score in a secure database file on the computer hard drive. Others administer the test and then print a copy of the student's test score upon request. By taking advantage of the online testing feature the teacher can eliminate the need to include the content of 
the CAI on an in-class examination all together. For programs that administer tests but do not record or print the test score, the teacher can test students' retention and at the same time reduce the amount of time they spend writing or selecting relevant test items by randomly selecting test questions from the embedded test to include as part of an in-class examination. Both strategies can be used to significantly reduce the time the teacher spends on testing without adversely affecting student learning.

Bloom and Trice (1997) noted the administration of an in-class test takes approximately 30 to 60 minutes of class time and then additional time to "go over" the test for review. In contrast, computerized testing takes approximately one quarter (Olsen, 1990) to one half the time it takes for traditional paper-and-pencil testing (Wise \& Plake, 1990). Even more significant, the time it takes for a teacher to write a test item that requires critical thinking can be as much as an hour or more (Bloom \& Trice). Most teachers use a combination of teacher-created items and questions selected from a computerized test bank or a text book publisher's test bank. All totaled, the time the teacher spends selecting or writing test items, compiling and administering the test, grading and analyzing students' scores and completing record-keeping tasks can either be eliminated or reduced using one of the testing strategies (Bloom \& Trice).

The research on the effects of testing by computer on students is sparse. Bloom and Trice (1997) noted computerized testing provides several advantages besides freeing-up class time such as promoting self-directed learning, allowing students to select the testing time, providing immediate feedback, and providing students with formative evaluations and, in some instances, remediation. Out-of-class testing also eliminates the problem of needing to capture the students' attention and direct it toward learning new concepts when their energies are still directed at the 
test they have just taken. Other advantages are greater standardization of the testing environment and possibly, increased reliability and validity of the test. This is particularly true of test instructions and may, or may not be true of the time allowed for testing depending on the testing situation (Bloom \& Trice).

Research regarding student anxiety in response to computerized testing shows conflicting results (Bloom \& Trice, 1997). An early study reported significant test anxiety and decreased performance in students who received computerized intelligence testing as compared with a control group that was tested via a paper-pencil form of the test (Llabre, Clements, Fitzhugh, Lancelotta, Mazzagatti, Quinones, 1987). In a study of college students, Dimrock and Comier (1991) found decreased performance on computer versions of the test but were unable to demonstrate a correlation between performance and either computer anxiety or computer literacy. Conversely, Wilson, Genco and Yager (1985) found no difference between groups of female college freshman in either test anxiety or test performance on computer versus paper-pencil testing. Likewise, Olsen (1990) was not able to demonstrate a difference in test scores in children who were tested on computers. Bloom and Trice found 127 junior and senior nursing students earned mean scores that were as good, if not better, than previous class scores on identical paperpencil tests $(\mathrm{p}<.05)$.

In summary, there are four groups of integration strategies that are primarily implemented by teachers to improve course productivity by reducing the time the teacher spends teaching or on teaching-related tasks. The issues surrounding the use of CAI for the purpose of saving the teacher's time are not straightforward. On one hand, teachers identified the most common reason they don't integrate CAI in their courses is because they lack the time to do so. The early 
planning and implementation stages can be so time-consuming, even for experienced computer users, that teachers can expect it to have a negative impact on their productivity for the first few years. However, once integrated, CAI can save the teacher considerable time and end up improving the productivity of the course as well as change the teacher's role from 'information provider" to "facilitator of learning" (Bates, 1999).

The fact that nursing education is so much more costly than other schools in higher education places nursing in a somewhat unique position. Since most of the cost in nursing education goes for the salaries of clinical instructors, anytime CAI can be used to replace some portion of clinical time, a substantial cost savings can result (Perciful \& Nester, 1996). Even though CAI is less cost-effective than any of the other instructional delivery systems, with careful planning and skillful course design, it can still be used to increase course productivity (Bailey, 1994).

There are different opinions about whether an individual, small-group or whole-class mode of instruction is more teacher time-efficient. Some believe the individual and small-group mode makes better use of the student's time and frees-up the teacher to help more students (Johnstone \& Maloney, 1998) while others found support for teacher time-reductions with both small-group and whole-class instruction (Goodman and Blake, 1996). Yanok (1988) found whole-class instruction was teacher time-efficient and effective. Kemp and Dayton (1985) noted the use of CAI for whole-class instruction has drawbacks but also eliminates repetition. They recommend using it to introduce new topics, for motivation, to illustrate relationships, to present new content and for enrichment (Kemp \& Dayton). Walvoord and Pool (1998) described the use of a series of A-V films that were used to provide a "first exposure" experience that helped 
students prepare written assignments, classroom discussions and unit exams. The time the teacher spend on the course was reduced by $20 \%$ while student enrollment was increased by $40 \%$ (Walvoord \& Pool).

The potential for cost savings also exists in the traditional classroom or clinical laboratory setting by using CAI to reduce teachers' time (Cambre \& Castner, 1993) but only if the CAI assignment is designated as a "required" part of the course (Bates, 1999). When the assignment is designated "optional," the price of instruction doubles and the productivity declines as the teachers' and students' time is duplicated (Bates).

Perciful and Nester (1996) reported CAI saved teachers an 8-hour day each rotation. Umlauf (1990) reported the use of CPR re-certification program not only provided staff nurses with around-the-clock accessibility, but also eliminated the need to have an in-service trainer present for teaching or to administer the performance tests. Jairath and Fitch (1994) used a CAI software protocol program to guide staff nurses in developing research proposals that not only reduced faculty time but also reduced the editorial effort required for the proposal development.

Teachers frequently use CAI programs to reduce the time they spend on testing by selecting software that administer embedded tests and either stores the students' scores in a secure data base file or provides a print-out of the test score. Other programs simply provide embedded tests that the teacher can use by randomly selecting questions for inclusion in an in-class examination. Either option can reduce the amount of time the teacher spends writing test items or administering the test all together. The usual time for an in-class test of between 30 to 60 minutes plus additional time to "go-over" it (Bloom \& Trice, 1997) can be cut by as much as one quarter to one half when the test is administered by computer (Olsen, 1990; Wise \& Plake, 1990). Other 
advantages of computerized testing are that they promote self-directed learning, allow students to select the testing time, provide immediate feedback, and eliminate the need to recapture the students' attention following an in-class exam. Other advantages may be greater standardization of the testing environment (Bloom \& Trice).

There are conflicting reports concerning the impact of computer testing on students. One early report showed significant anxiety and decreased IQ test performance (Llabre et al., 1987) while another showed decreased performance on the computer version of a college level test but was unable to demonstrate a correlation with anxiety or computer skills (Dimrock \& Comier, 1991). In two other studies, researchers found no difference in performance between groups of college freshmen (Wilson, Genco \& Yager, 1985) and none in children who were tested by computers (Olsen, 1990). Bloom and Trice found junior and senior nursing students' scores were as good, if not better, than the scores of a previous class on the same test.

\section{Types of Software}

The fourth study question sought to determine the types of software nurse educators use to teach clinical decision-making. Following that line of thought, the next topics will discuss what the literature says about simulations, tutorials, virtual reality, expert systems, and drill-andpractice programs. Next, the discussion will focus on what the literature says about commercially purchased and custom-produced and teacher-authored software.

\section{Five Types of Software}

The 1993 edition of Bowell's “Directory of Software for Nursing Education,” identified a large majority of the titles as text-based simulations (96\%), a small number of tutorial programs (2\%) and only two virtual reality programs (Bowell,1993). In the most recent 1995 edition, most 
of the software programs on the market were still text-based case study simulations (70\%) but a larger number were graphic simulations $(20 \%)$ and tutorial programs $(6 \%)$. Virtual reality and expert systems software programs (4\%) were also beginning to make strides in availability (Bowell, 1995).

\section{Commercially and In-house Produced Software}

Generally, nursing educators have three sources to chose from when selecting software that is designed to teach students clinical decision-making skills. Most of the programs are purchased from commercial software companies that are either exclusively devoted to software production or are branches of the major nursing textbook publishers. The other two sources are custom-produced software or teacher-authored programs. The discussion that follows will begin with commercially purchased and then discuss the benefits and drawbacks to custom-produced and teacher-authored software.

The major advantage of commercially produced, pre-programmed CAI software is that it is much less expensive to purchase than customized software (Bates). Bowell (1995) noted the use of CAI substantially increased between 1990 and 1995 and Edwards (1995) noted the quality of the programs improved dramatically over the same time period. In the 1993 edition of Bowell's "Directory of Software for Nursing Education," there were over 600 titles and 70 vendors listed. In the next edition of the directory published in 1995, there were only 300 titles and 22 vendors. The declining numbers reflected a trend in the industry as a whole away for semi-professional developers toward the expansion of a few commercially owned software companies and branches of the major nursing textbook publishing companies that were producing high-quality software. According to Edwards, many nursing teachers and semi-professional 
software producers simply disappeared from the marketplace when they found they could not compete with the more sophisticated commercially produced programs.

Most of the available resources that describe the costs and benefits of the various types of software being used in higher education agree that custom-produced software is the highest quality but is also the most expensive of the three options. For instance, Bates (1999) noted customized software that is produced in-house by a team of experts is more expensive that any other educational delivery system. The production costs are high regardless of whether the software is developed by a team of specialists who are employed by the school or by outside private consultants that are hired specifically for the project. The reason is for the high cost is that the team is typically composed of a number of highly skilled, and highly paid professionals. A team is typically made up of one or more faculty members or a content expert, a computer programmer, an instructional designer, graphic artists, and several members of a media production staff. Another reason it is so expensive is that the development process takes so long and requires a coordinated effort by all the members of the team all the way through. While using in-house specialists may reduce the costs in terms of salaries, the major drawback to the in-house approach is that the coordination and logistics of the project are much more difficult since the team members are typically engaged in many other projects at the same time (Bates, 1999).

Bates (1999) noted that there are several ways the cost-effectiveness of customized software can be improved such as developing the instruction in discrete modules so that revisions and updates can be made easily and cheaply when needed, modules can be developed for use by multiple users such as different groups of learners (i.e., on-campus or distance learners), or they can be developed for sale to learners enrolled in certification programs, continuing professional 
education, or as stand-alone instruction that can be sold to employers, hospitals, or other schools (Bates). Fisher (1997) noted cost-effectiveness can be improved by using a large storage capacity media, such as DVD, so that several versions of the same multimedia course can be burned to the same disc. Each version might present slightly different content to accommodate different learning styles or be written for learners with different levels of expertise (Fisher).

The literature is mixed regarding recommendations and reviews of faculty-produced CAI software. Gleydura et al. (1995) believed the availability of "authoring," and presentation software that came on the market in the early and mid 1990s was an open door for nursing teachers to create their own interactive multimedia training tools. Rouse (1999) predicted the rapid decline in the costs of computer hardware and software developments that made in-house CAI production easier and affordable would translate into most nursing teachers creating their own educational training tools in-house. She recommended concentrating on difficult concepts that students would need to access a number of times throughout the curriculum, content that must be repeated several times to small groups of students, or topics that were difficult to present in an interesting manner using didactic methods (Rouse). Calderone (1994) thought there would be so many teachers using authoring software to develop their own CAI that it would put pressure on commercial companies to reduce the costs of commercially produced software.

On the negative side, Kilmon (1996) and Edwards (1995) noted that CAI development projects undertaken by individual faculty were often of much lower quality than customized or commercially purchased programs. Michelman (1994) warned faculty members who considered developing CAI for their courses to be aware that multimedia development was very costly in terms of faculty time. Learning curves were steep and delving into software development would 
mean sacrifices in other areas. For that reason, she warned teachers who were employed at schools where they are not rewarded with incentives such as reductions in their teaching loads or credit toward promotion and tenure would be better off to wait until the software became available commercially (Michelman). Gleydura et al. (1995) also noted teacher-authored software may actually end up costing more than commercially produced programs because the work and time involved was so extensive. They also noted the programs usually met the educational needs of the course better than what was available commercially and they thought it was worth the extra effort and expense (Gleydura, et al.). After deTornay and Thompson (1982) went through the process of developing a single CAI software program, they concluded it was too time-consuming and provided too few rewards to recommend it to other nurse educators and Edwards (1995) noted few nursing teachers ever produce a second CAI project. On the other hand, the major advantage cited of purchasing commercially available software is that most programs are high quality and polished products before they are released. One disadvantage is that the programs may present contextual or cultural bias which means the content may not be applicable to all countries or regions of the United States (deTornay \& Thompson).

Engberg and White (1998) designed a series of teacher-authored video-enhance CAI programs because they could not find a program available commercially that incorporated the problem-based learning (PBL) curriculum they preferred to use at their school. The program allowed advanced practice nursing (APN) students to use the key board to query simulated patients about current health problems, past medical history, family history, and conduct a review of systems. Students also entered free-text questions to request video-taped parts of a physical exam and a variety of diagnostic tests. The program provided both feedback and remediation to 
correct students' misconceptions. The students used the series of programs throughout the curriculum for a variety of courses and to take interactive tests. After the didactic content was presented in the classroom, students were assigned to complete a CAI unit of instruction. Students' evaluations of the software were extremely positive and a cost analysis showed it was much less expensive than the traditional strategy of using live actors who portrayed simulated patients (Engberg \& White).

In summary, there are no recent publications that describe the types of software being used by schools of nursing in the U.S. The types of commercially available software changed during the years from 1993 until 1995 from almost all text-based case study simulations and a small number of tutorials to include some graphic simulations and tutorials. Virtual reality and expert systems programs also made small strides during the time period (Bowell, 1995).

The three sources of software programs that are being used by nurse educators to teach clinical decision-making are commercially produced, custom-produced, and teacher-authored programs. Of the three, customized software is the most expensive because it is developed by a team of specialists. The team is composed of a group of highly qualified professional and technical specialists. The two problems that make the cost prohibitive for most schools are the costs of the salaries of outside consultants and the difficulty coordinating the work of in-house experts who are usually involved in many different projects at the same time (Bates, 1999). Customized software can be designed to be more cost-effective by developing the instruction in discrete modules so updates can be made cheaply and easily. The costs can also be diffused by designing the modules for multiple users such as certification programs, continuing professional education, or for sale to employers or other schools (Bates). Another strategy is to develop 
several versions of the same instruction for various levels of learners that can be stored on a high capacity storage device such as a DVD (Fisher, 1997).

Commercially-produced software is expensive when compared with other instructional media but much less expensive than custom designed software. The use of commercially produced software increased substantially between 1993 and 1995 (Bowell, 1995) and the quality of the programs improved dramatically during the same period. However, the number of titles available on the market declined by half and the number of software vendors dropped from 70 to 22. Edwards (1995) attributed the decline to the withdrawal by a large number of faculty and semi-professional developers who left the field when they could no longer compete with the more sophisticated programs developed by professional software companies.

The literature presents mixed opinions about the value and ease with which faculty can produce their own software. Several believed the introduction of authoring and presentation software in the early and mid 1990s would mean teachers could and would produce their own customized CAI (Gleydura et al., 1995; Rouse, 1999). Some even believed faculty productivity would put pressure on software companies to reduce their prices (Calderone, 1994) while others noted CAI projects by faculty were often low in quality (Edwards, 1995; Kilmon, 1996). Several authors wrote warning other faculty of the costs in terms of time and difficulty (Michelman, 1994). Gleydura et al. (1995) noted teacher-produced programs usually meet the needs of course better but the time involved could be extensive. Engberg and White (1998) described a series of CAI programs they developed for use in a PBL curriculum that met their students' needs better and less-expensively than the actor patients they used before. 
Next, the review will discuss the literature concerning the third study question that addresses the key features of schools and faculty who are perceived by participants as integrating CAI effectively. The first topic will be key features of schools and the second, key features of the teachers.

Key Features of Schools and Faculty That Effectively Integrate CAI

\section{Key Features of Schools}

Several researchers have looked at the features of the schools and teachers who integrate CAI effectively. Cambre and Castner (1993) conducted a large survey of 1120 schools of nursing and completed personal interviews with 122 school administrators to determine the characteristics of schools that were most likely to successfully integrate computer technology. They identified two levels of indicators of success; the first was at the institutional level and the other was at the level of the individual faculty member. It was interesting to note that the two were not necessarily present at the same school (Cambre \& Castner).

The single indicator of success at the faculty level Cambre and Castner (1993) noted was a high degree of teacher enthusiasm. At the institutional level, successful implementation was defined by the breadth and depth of the use of the technology. For instance, many schools reported the same software was used in three or four ways throughout the curriculum by more than one teacher and that there were high levels of user satisfaction and student achievement. The researchers described a successful campus as one where the school might only own five copies of two programs, but they were almost always in use. The CAI was used to replace the educators' labor, most often to replace lecture, and the same content was almost never taught twice except for remediation purposes. In the clinical laboratory setting, students completed the CAI programs 
in small groups and then went back to review and practice individually. Surprisingly, smaller schools actually had a better chance of integrating CAI than bigger ones. In fact, many large, well-equipped institutions reported very low use of the technology. The amount of space, financial investment and allotted personnel were not strong indicators of successful integration (Cambre \& Castner).

Carty and Rosenfeld (1998) survey was conducted to determine the status of computer technology in nursing education and included 190 schools of nursing from across the U.S. The respondents were a proportionate mix of diploma, associate, and baccalaureate and higher programs that were similar to those in the general population of schools of nursing in the U.S. A significant component of the survey addressed an inventory of technology resources available at each institution. The findings revealed almost all respondents $(97.8 \%)$ reported computers were available for students to use at their schools. They were available in learning resource centers (58\%), nursing labs (47\%) and other locations (17\%), such as labs where students shared the computers with students from other departments. Without exception, baccalaureate and higher programs reported having more computer resources (i.e., computers, computers in faculty members' offices, a LAN, and Internet access) than diploma or associate degree programs. When reporting on the number of computers available for students' to use, diploma schools had a mean of 14 , associate degree programs had a mean of 13 and baccalaureate programs had a mean of 30 computers (Carty \& Rosenfeld).

The survey also contained a number of questions about software applications in the nursing curriculum (Carty \& Rosenfeld, 1998). Computer-assisted instruction was the most prevalent educational application used and there was almost universal use by all the schools. 
More than half (61\%) of the schools used some form of IVDI, 33\% used testing applications, and $52 \%$ used programs that were stored on CD-ROMs. Respondents were asked to identify which types of computer-based clinical applications were available for students in the lab or simulated setting. The results revealed $69 \%$ indicated they used simulations for assessments, $63 \%$ used them for medication administration, and $46 \%$ used them for patient documentation. Though a clear majority of all program types used them, diploma programs reported greater use of simulated CAI programs for clinical applications than their counterparts (Carty \& Rosenfeld).

The data analysis also revealed the schools reported major problems with their technology budgets (Carty \& Rosenfeld, 1998). There was a huge variance between what programs spent for technology with a range of from $\$ 0$ to $\$ 156,000$ annually. Most schools allotted approximately $\$ 1,000$ a year. Participants reported lack of funding was a major barrier with $70 \%$ reporting they perceived they had less than adequate funding for computers and software purchases. Similarly, in the area of personnel costs, only 70 respondents reported their schools' budget contained specific funds for computer technology operations in the previous year. The mean amount that was devoted to the costs for personnel was $\$ 8,343$; BSN programs reported an average of $\$ 13,879$ and associate degree and diploma programs reported almost identical averages of $\$ 4,200$. The researchers concluded the data suggested the majority of nursing programs do not provide enough financial support for technology and related personnel. The finding was corroborated by a response of $80 \%$ of the participants who indicated costs were the major barrier that kept them from accomplishing the technological objectives they would like to have set for their nursing units (Carty \& Rosenfeld).

MacKnight (1995) noted a trend toward in higher education toward increased 
decentralization with more schools opting for client/server architecture and multi-platform integration so that more computing was being done at the departmental level. Most processing was moving away from big mainframe computers to lower-cost desktop computers so that departments were better able to meet their own computing needs. In addition, MacKnight noted centralized computer labs were expected to disappear from college campuses as students purchase their own portable lap-top computers that would be used to access instruction anywhere, anytime over telephone lines and through wireless networks.

\section{Key Features of Teachers}

Bass (1991) surveyed 320 nurse educators from Georgia to identity important discriminating features among those who did and did not adopt CAI. The findings indicated the majority of nurse educators who adopted the technology had more teaching experience, taught clinical nursing subjects, had more knowledge about microcomputers and CAI, obtained most of their information through interpersonal communications with other technology using teachers, perceived the benefits of CAI outweighed the difficulties inherent in its use, and had a high degree of decision-making autonomy in their classrooms. The perceived level of administrative support available for the adoption of CAI was found to be neither a necessary, nor sufficient condition to adopt but lack of support was found to be an important predictor of non-adoption. No significant differences were found among adopters and non-adopters based on the teachers' primary role (classroom teacher, clinical instructor, researcher or administrator), level of instruction (associate, baccalaureate, masters, doctoral), type of institution (public or private school), acquisition of knowledge about CAI through mass media communication, attitude toward CAI, or willingness to use CAI. It was noted the attitudes toward and willingness to use CAI 
were extremely positive for all study participants and therefore, could not be used to discriminate adopters from non-adopters (Bass).

Cambre and Castner (1993) conducted interviews with 193 nursing school administrators, teachers, and students and did 15 site visits to schools to determine the characteristics of schools and faculty who successfully implemented computer technology. They found that while technology-using teachers typically worked for smaller schools and that larger schools often had difficulty achieving institutional success, individual faculty members who worked for larger institutions were sometimes extremely successful at implementing technology. In most instances, it was one faculty member who usually acted as a catalyst for bringing the technology to the school and keeping the computers up and running. Successful integrators used the technology in three or four different ways and assisted other teachers in adopting it so that the software was being used throughout the curriculum (Cambre \& Castner)

Other findings from the literature indicated technology-integrating teachers not only spend a good deal of personal time working with computers but they also have more extensive computer training and teaching experience (Marcinkiewicz, 1993). They are surrounded by other teachers who used computers for meaningful activities and had administrative support for technology use (Becker, 1994) and sufficient staff development opportunities (U.S. Congress, Office of Technology Assessment, 1995). The level of comfort faculty reach integrating technology was not achieved through a single event but came through continuous exposure, encouragement, and support from colleagues, students and academic support personnel (MacKnight, 1995). Hadley and Sheingold (1993) noted the teachers in their study took advantage of many opportunities; they participated in both in-service offerings on their own time and almost 9 of 10 indicated that 
to some degree, they were self-taught. Close to eight out of ten participants indicated they attended conferences and workshops on their own time. About 6 in 10 had taken in-service training offered at the school where they worked and about 4 in 10 had taken courses in their graduate and undergraduate schools or had received instruction from other teachers or consultants (Hadley \& Sheingold, 1993). Ingram (1994) noted the strong tendency for teachers to teach as they have been taught is difficult to overcome and usually only happens through formal education when computers are integrated into the course. Teachers need to be exposed to technologyaugmented teaching in order to be convinced of the benefits and to see how it is done (Ingram). Based on this same assumption, Lewis and Watson (1997) designed a series to faculty workshops to introduce the faculty at their school of nursing to five computer applications that are commonly used for education. In a follow-up article, Lewis, Watson and Newfield (1997) extended the introductory course on computer basics to an on-going series of computer training workshops that ran over a three year period. Reiser (2002) also noted teachers who effectively integrated technology either attended in-service or post-graduate education courses.

Peirson (2001) found teachers who were good technology integrators planned for regular computer use and maintained higher expectations for student learning from CAI than teachers who only used it occasionally. They also believed they could use computers to address individual learning needs and were willing to shift their focus to activities that were student-centered (Hadley \& Sheingold, 1993) with less whole-group instruction and more independent work (Waxman \& Huang, 1996). They also had enough technology skills to be able to troubleshoot common technological challenges, use the computer to simplify administrative duties and to teach students how to operate the hardware and software (Peirson). 
In a nationwide survey study, Hadley and Sheingold (1993) looked at public school teachers (primary and secondary) who effectively integrated computers into their teaching and found teachers who used computers most were a more mature, and more experienced bunch: over half were between 40 and 49 years old and three fourths of them had been teachers for 13 years or more. In terms of gender, the group was more equally distributed than public school teachers overall: $58 \%$ were women and $42 \%$ were men in contrast with $68 \%$ women and $32 \%$ men nationally. Elsewhere, the literature suggests teachers who integrate CAI into their courses may be younger than those who do not. The thinking is that younger teachers have been exposed to computers more in a formal education setting, and as a result, would be more comfortable using them to teach than teachers who have been out of school longer. Lewis, Watson and Newfield (1997) indicated the age of faculty and administrators was one of the difficulties encountered when implementing technological advances in an academic setting. They also noted they needed to change the focus of a basic computer literacy course that was offered to graduate and undergraduate nursing students to a more advanced topic of "nursing informatics" because the "students who enrolled in the course over the three-year period were increasingly computer literate and were interested in a more advanced course" (Lewis et al., p. 188).

In summary, this final section of the literature review looked at the key features of schools and teachers who integrate CAI effectively. Several authors noted teacher enthusiasm was a major key to successful implementation at the faculty level (Bass, 1991; Cambre \& Castner, 1993; MacKnight, 1995). At the school level, the picture is more complex. The schools that integrated CAI most effectively were often smaller schools. In fact, many large and wellequipped schools seldom used the technology. The amount of space, financial investment and 
allotted personnel were not strong indicators of successful integration. Schools that were strong integrators might only own a few software programs but they integrated them into the curriculum at several different points. They used CAI to replace the teacher, often to replace lecture. In the clinical laboratory setting, students often used CAI in small groups and then went back to review the instruction and practice individually. (Cambre \& Castner). In another study, Carty and Rosenfeld (1998) found almost all nursing schools in the U.S. had computers available for students to use and over half had a computer lab in the school of nursing. Baccalaureate schools had more computer resources and twice as many computers as diploma or associate degree schools. In terms of software applications in the nursing curriculum, CAI and IVDI were used almost universally by all the schools, but here again, diploma schools reported using CAI for clinical applications more often than BSN or ADN schools. In terms of technology budgets, there was a wide variance between what schools spent for technology applications but most schools allotted approximately $\$ 1,000$ a year. Reports of the amount schools spent on support personnel indicated BSN programs spent well over twice as much as associate and diploma programs. Almost all the schools (80\%) indicated a lack of funds for technology was a major barrier that kept them from accomplishing their technology related objectives (Carty \& Rosenfeld).

From another perspective, MacKnight (1995) noted the trend in higher education is toward the decentralization of computer departments so that more computing is done at the departmental level. Another facet of the decentralization process is that centrally located computer labs are expected to disappear as most college students purchase their own computers, and mobile lap tops and are able to access the schools' software from anywhere, anytime over the Internet (MacKnight). 


\section{CHAPTER III}

\section{METHODOLOGY}

\section{Selection of Participants}

\section{Proportionate Mix of Schools}

The sample of 140 schools, which was $10 \%$ of the total population, was randomly selected from a comprehensive list of the 1,398 schools of nursing in the United States. Based on the literature findings that describe the population as being composed of a diverse mix of schools that include large, publically-supported universities and community colleges as well as small, privately-owned schools, it seemed reasonable to assume that there were significant differences not only in the size but also in the financial status and computer resources of the schools. For that reason, a stratified random sampling technique that was based on a proportionate mix of the total population was drawn so that the sample would consist of $37 \%$ from baccalaureate degree schools (BSN), 58\% from associate degree (AD), and 5\% from diploma schools.

\section{Proportionate Mix of Geographic Regions}

In addition, to ensure the sample represented all geographic areas of the country equally, a map of the United States was divided into six geographic regions from which the stratified sample was randomly selected using a table of random numbers (See Tables 1 and 2). 
Integration CAI 130

Table 1. Percent and Number of Each Type of School in the Population and the Sample

\begin{tabular}{lccc}
\hline Program & Schools in Population & Percent & Schools in Sample \\
\hline Bachelor & 520 & $37 \%$ & 51 \\
Associate & 804 & $58 \%$ & 81 \\
Diploma & 74 & $5 \%$ & 8 \\
\hline Totals & 1,398 & $100 \%$ & 140
\end{tabular}

Table 2. Randomized Selection of Schools from Six Geographic Divisions

\begin{tabular}{lcccc}
\hline North West & Bachelors & Associate & Diploma & Total \\
\hline 1. Alaska & 1 & & & 1 \\
2. Washington & 5 & 20 & & 25 \\
3. Oregon & 4 & 14 & & 18 \\
4. Idaho & 5 & 4 & & 9 \\
5. Montana & 3 & 4 & & 7 \\
6. Wyoming & 1 & 6 & & 39 \\
7. N Dakota & 7 & 29 & 3 & 10 \\
8. S Dakota & 5 & 5 & & 22 \\
9. Wisconsin & 9 & 13 & & 26 \\
10. Nebraska & 6 & 6 & 1 & 70 \\
11. Minnesota & 9 & 17 & & \\
12. Illinois & 24 & 42 & 4 & 247 \\
& & & & 23 \\
\hline Total Schools & 79 & 160 & 8 & 1 \\
\hline Selected from Region & 8 & 14 & &
\end{tabular}




\begin{tabular}{lcccc}
\hline Mid-North & Bachelors & Associate & Diploma & Total \\
\hline 1. Michigan & 16 & 32 & & 48 \\
2. Indiana & 19 & 7 & 2 & 28 \\
3. Iowa & 12 & 15 & & 27 \\
4. Ohio & 22 & 29 & 6 & 57 \\
5. Pennsylvania & 31 & 22 & 24 & 77 \\
\hline Total Schools & 100 & 105 & 32 & 237 \\
\hline Selected from Region & 9 & 13 & 2 & 24
\end{tabular}

\begin{tabular}{lcccc}
\hline North East & Bachelors & Associate & Diploma & Total \\
\hline 1. Connecticut & 7 & 6 & 1 & 14 \\
2. New York & 43 & 47 & 1 & 91 \\
3. Vermont & 2 & 4 & & 6 \\
4. Maine & 5 & 6 & & 11 \\
5. N Hampshire & 3 & 3 & & 6 \\
6. Mass & 11 & 19 & 2 & 32 \\
7. R Island & 3 & 2 & 1 & 6 \\
8. Delaware & 2 & 3 & 1 & 6 \\
9. New Jersey & 16 & 12 & 10 & 38 \\
10. Maryland & 8 & 14 & & 42 \\
11. Wash DC & 3 & 1 & & \\
& & & & 236 \\
\hline Total Schools & 103 & 117 & 16 & 24 \\
\hline Selected from Region & 9 & 13 & 2 &
\end{tabular}


Integration CAI 132

\begin{tabular}{lcccc}
\hline South West & Bachelors & Associate & Diploma & Total \\
\hline 1. Hawaii & 4 & 4 & & 8 \\
2. California & 16 & 72 & & 88 \\
3. Nevada & 2 & 4 & & 6 \\
4. Utah & 5 & 6 & & 11 \\
5. Arizona & 5 & 9 & & 14 \\
6. Colorado & 6 & 12 & & 18 \\
7. New Mexico & 2 & 7 & 2 & 9 \\
8. Texas & 17 & 45 & & 64 \\
& & & & 218 \\
\hline Total Schools & 57 & 159 & 2 & 22
\end{tabular}

\begin{tabular}{lcccc}
\hline Mid-South & Bachelors & Associate & Diploma & Total \\
\hline 1. Oklahoma & 10 & 15 & & 25 \\
2. Arkansas & 11 & 7 & 3 & 21 \\
3. Missouri & 16 & 22 & 1 & 39 \\
4. Louisiana & 12 & 7 & 1 & 20 \\
5. Mississippi & 7 & 14 & & 21 \\
6. Tennessee & 18 & 13 & 1 & 32 \\
8. Kansas & 11 & 18 & & 29 \\
9. Kentucky & 12 & 23 & & 25 \\
& & & & 23 \\
Total Schools & 97 & 119 & 6 & \\
\hline Selected from Region & 9 & 13 & 1 &
\end{tabular}




\begin{tabular}{lcccc}
\hline South East & Bachelors & Associate & Diploma & Total \\
\hline 1. West Virginia & 9 & 10 & & 19 \\
2. Virginia & 12 & 17 & 7 & 36 \\
3. North Carolina & 12 & 46 & 3 & 61 \\
4. South Carolina & 8 & 12 & & 20 \\
5. Alabama & 12 & 19 & & 31 \\
6. Georgia & 16 & 15 & & 31 \\
7. Florida & 15 & 25 & & 40 \\
\hline Total Schools & 84 & 144 & 10 & 238 \\
\hline Selected from Region & 8 & 14 & 1 & 23
\end{tabular}

The Instrument

The Integration of Computer Assisted Instruction Questionnaire

Since no suitable instrument could be found, the Integration of Computer-Assisted Instruction (ICAI) Questionnaire (See Appendix A 1 for a copy of the Integration of Computerassisted Instruction Questionnaire) was developed as the measurement tool for this study. It was designed by the researcher and was based on integration strategies gleaned from a review of the literature (Bates, 1999; Dick \& Carey, 1991; Gagne', 1985; Gustafson \& Powell, 1991; Kemp et al., 2001). The ICAI took participants approximately 20 to 25 minutes to complete. It was designed to be appropriate for administration to participants who were knowledgeable about computer-assisted instruction and educational strategies and who were employees of the school that worked with teachers and students who used CAI to teach \learn clinical decision-making. Participants were also recruited who were interested in participating in the study but did not use CAI in order to gather a more realistic assessment of what was actually being done in field of nursing education. Participants who did not use CAI at their school only completed the demographic portion of the questionnaire (Questions 11 through 23). 
Integration CAI 134

\section{Administration of the Instrument}

The ICAI questionnaire was an electronic interactive document that was made available

to participants over the Internet. A web-based survey instrument was chosen because it offered a number of advantages over a mailed survey. Not only was the questionnaire easier and less expensive to distribute but it was interactive and was designed to provide participants with guidance and help exactly where it was needed without having to refer the respondent to a separate form. For each of the self-report items (e.g., "how often did teachers at your school use each of the following integration strategies," or "based on your experience, do you agree or disagree that the following integration strategies are effective for increasing student learning?") the respondents were asked to point and click with a mouse on a radio button to indicate his/her response. Other open-ended questions asked the respondents to type in the exact number to indicate how many (e.g., "of each type of software did teachers at your school use during the past year?"or "how many full and part-time technical assistants are available in the computer lab?"). The questionnaire ended with a single, open-ended question which asked respondents to type comments they would like to make that might shed light on the study.

\section{Validity and Reliability of the Instrument}

Three experts in the area of nursing education (see Definitions of Terms for a description of the experts' qualifications) independently reviewed the instrument, made recommendations and after several rounds of revisions indicated that, in their opinion, it had both content and construct validity. Additionally, an expert in the design of on-line questionnaires (see Definitions of Terms for an operational definition of the expert's qualifications, Page 155) also reviewed the questionnaire for design flaws and indicated the form was operationally and 
Integration CAI 135

technically sound. A pilot study that included five subjects was conducted in order to detect any flaws in data collection. The results of the pilot study revealed the data collection program that was written by the computer programmer-consultant performed as expected.

\section{Study Design}

A descriptive study design was selected as the most appropriate research design for this initial work in the area (Gay, 1992). A survey method was chosen recognizing it would mean a sacrifice of some of the depth and texture that a qualitative study would provide, but that it would also provide the broader picture of the educational practices of many nurse educators from across the U.S. that was needed. The study was also intended to answer questions concerning the computer resources and types of software nurse educators currently used to teach clinical decision-making and which types their peers were able to integrate most effectively. Demographic data was also collected to assist in describing the results of the participating schools and respondents.

\section{Procedure}

Once a school was randomly selected from a comprehensive list of schools using a table of random numbers, the school's administrator or a representative from the school whose Internet address was made available at the schools' web site was contacted by an initial e-mail note (See Appendix A 2 for a copy of the initial contact note) requesting they either pass the note along to a potential participant, or identify the e-mail address of a person who might be interested in participating in the study (Schaefer \& Dillman, 1998). The initial contact note described a likely participant as a school employee who was either actively engaged with teachers and students in the use of CAI for instruction in decision-making, or if the school did 
not use CAI, someone who was an employee of the school who would be willing to participate in the study. As soon as 140 participants were identified, a bulk cover e-mail letter (See Appendix A 3 for a copy of the cover letter) was sent to all the participants explaining the study more fully and providing them with the Internet address of the questionnaire at http://www.dalesplace. net/survey/CAI Questionnaire.htm. The address was the Universal Resource Locator (URL) of the web page that hosted the survey. When the respondent connected to the host server, the first two opening pages explained their rights as participants in an IRB approved study and required that they click a radio button giving their informed consent and to indicate their willingness to participate. The third page provided directions for how to complete the ICAI questionnaire. The cover e-letter also contained a five-digit code number that was assigned to each participant that they were required to type at the end of the form in order to submit the questionnaire. The purpose of the code was to ensure that only those who were invited to participate in the study did so, but it was also used to identify, without breaking confidentiality, when participants completed the questionnaire so that only those who failed to respond by the deadlines received follow-up reminder messages. Respondents were asked in the first cover e-mail letter to complete and submit the survey before the end of a three-week period. Those who did not meet the deadline were sent a reminder e-message that contained the same information as the first (See Appendix A 4 for a copy of the reminder e-mail message). At that time, respondents were asked to complete the questionnaire within a two-week period. Those who still failed to respond were sent a second reminder e-mail message with the same message as the other two. The thirty-one volunteers who still did not respond were contacted after the data collection period ended and 
Integration CAI 137

asked to provide information for a non-response bias-check. Seven of the 31 non-responders did So.

The web-page consisted of a form that contained the survey of questions and used Java Script to validate key fields. The page also provided links to definitions of key terms used in the survey, as well as a button they could click to return to their current place in the questionnaire after they viewed a definition. After a respondent completed and submitted the survey, data was sent to a non-public e-mail box via a Common Gateway Interface (CGI) script to the host server. It did not rely on a local e-mail client which was done to ensure the data was not intercepted or modified in any way. The survey data included a method that permitted the researcher to verify the uniqueness and source of each data set. Data returned from the Internet survey was parsed, extracted and stored in comma-delimited format using a customized program designed for that purpose. The data was directly importable by the Corel Quattro Pro data processing software that was used for the statistical analysis. Respondents also had the option of printing the questionnaire and sending it back to the researcher by postal mail. Directions for where to send it were available on a Acrobat Adobe PDF form of the questionnaire that was available by clicking a button on the opening page of the questionnaire. Only one respondent did so.

\section{Analysis of the Data}

Scores from the CAI Questionnaire were used for the data of this study. Measurements of central tendency were used to describe specific points of distribution of the data on the measurement scale (e.g., mean, mode and median), and frequencies and percentages were used to describe the findings. Measures of the standard deviation were used to indicate the spread of the data on the scale of measurement. Pearson product-moment correlation coefficients were 
calculated to determine the relationships between the frequency of use of the integration strategies and the perceived effectiveness of each strategy in terms of student learning, decreasing costs, students' time and the teachers' time. Pearson product-moment correlation coefficients were also used to determine if a relationship existed between several of the descriptive variables (e.g., type and size of school, number of computer resources, etc.) and the frequency of use and perceived effectiveness of the integration strategies. 
Table 3. Summary of Study Questions, Questionnaire Questions Answered and Methods of Analysis

\begin{tabular}{|c|c|c|}
\hline Study Questions & Data Collected & Methods \\
\hline 1. What integration & a. List of 19 integration strategies & 1) Review the literature, \\
\hline strategies have nurse & compiled from the literature included in & compile a list of integration \\
\hline educators used to & the CAI Questionnaire. & strategies that were included \\
\hline integrated CAI programs & b. Likert scale ratings of nurse & in CAI Questionnaire. \\
\hline designed to teach clinical & educators' perceptions of how often & 2) Calculate frequencies, \\
\hline decision-making into the & they and colleagues used each & measures of central tendency \\
\hline \multirow[t]{4}{*}{ courses they teach? } & integration strategy over the past year. & and percentages. \\
\hline & (Answers Q 1 \& 2) & 3) Discuss relationship \\
\hline & & between frequency of use and \\
\hline & & literature recommendations. \\
\hline 2. How effective do nurse & a. Likert scale ratings of nurse & 1) Calculate means $\backslash$ medians, \\
\hline educators perceive the & educators' perceptions (1-4) of how & standard deviations, \\
\hline integration strategies have & effective each integration strategy has & percentages for entire sample. \\
\hline been in terms of & been in terms of & 2) Pearson $r$ correlations \\
\hline a. student learning, & student learning, (Answers Q3) & between frequency of use and \\
\hline b. students time to learn, & student time to learn, (Answers Q5) & perceived effectiveness of \\
\hline c. cost savings, & costs, (Answers Q4) & each strategy. \\
\hline d. teachers' time? & teachers' time. (Answers Q6) & \\
\hline
\end{tabular}


3. What are the key

features of the schools and

educators that are perceived

by participants to integrate

CAI designed to teach

clinical decision-making

effectively? a. Descriptive demographic data about

schools in the sample (e.g., type of

school, institution, size, computer

resources, number and percent of

teachers who use CAI, etc.).

(Answers Q 11-18 schools)

b. Descriptive demographic data about

participants (role, gender, age,

educational background).

(Answers Q 19-23 participants)
1) Calculate totals, percentages, measures of central tendency, standard deviations

2) Calculate totals, percentages, means

3) Pearson r correlation between descriptive variables of schools and frequency of use

4) Pearson $r$ correlation between descriptive variables (e.g., type of schools) and perception of effectiveness of integration strategies

4) Pearson $r$ correlation between descriptive variables (educators) and frequency of use

5) Pearson r correlation between descriptive variables (participants) and perceived effectiveness of strategies 


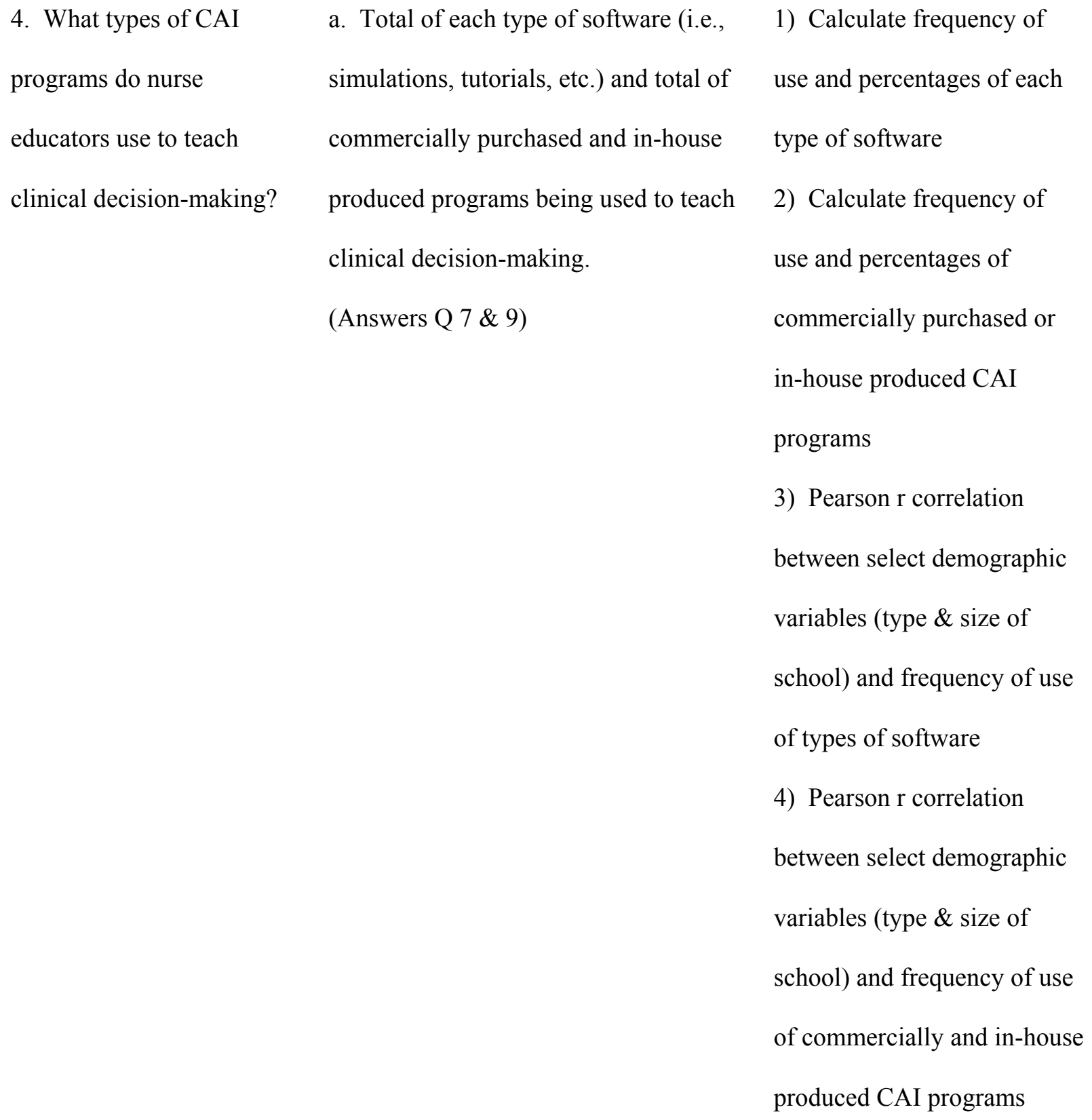




\begin{tabular}{|c|c|c|}
\hline 5. How effective do & a. Likert scale ratings of educators' & 1) Calculate totals and \\
\hline participants perceive & perceptions of how effectively teachers & measures of central tendency \\
\hline teachers at their school are & at their schools are at integrating each & (mean, median, mode \& SD) \\
\hline at integrating the different & type of CAI software into the courses & of Likert scale ratings of \\
\hline types of CAI software & they teach. & educators' perceptions of \\
\hline (simulations, tutorials, etc.) & (Answers Q 8 \& 10) & how effective teachers at \\
\hline and commercially & & their schools are at \\
\hline purchased and in-house & & integrating each type of \\
\hline produced software into the & & software. \\
\hline
\end{tabular}

\section{Assumptions Underlying the Study}

1. It was assumed that the size of the school was an important variable that would impact the findings of this study. Based on the extremely dated, but most recent national study of schools of nursing throughout the United States (Carty \& Rosenfield, 1998), BSN schools have more computers than either AD or diploma programs. Also based on general knowledge, universities and community colleges are publically funded and often larger institutions that have more computer resources (computers, software, and support personnel) than smaller or privatelyowned schools. For those reasons, a stratified random sampling technique was used to select the subject schools for this study. Data was collected to see if a relationship exists between the type of program, the school size, and the number of computers, and other computer resources.

2. It was assumed that nurse educators were using the integration strategies that were 
included in the questionnaire and that some strategies were being used more often and were perceived to be more effective than others.

\section{Limitations of the Study}

1. A serious limitation to the study was the small number of diploma schools available nationwide for inclusion in the study.

2. It was necessary to limit the number of integration strategies included in the questionnaire to a few pre-instruction, mid-instruction and post-instruction strategies. Integration strategies were selected for inclusion in the study based on those that were judged by the researcher as most relevant for impacting the productivity of the course based on findings from the literature review. The reason for limiting the number of questions was to make the task of completing the questionnaire appear less daunting to respondents. Dillman (2000) noted that care should be taken to limit the number of questions to those that are most relevant to the study in order to maximize the number of responses.

3. It was deemed appropriate to limit the number of subject schools to $10 \%$ of the total population because it is the recommended survey sample size (Gay, 1992) and due to resource and time-constraints.

\section{Definition of Terms}

1. Computer-assisted instruction (CAI) refers to software programs that employ various strategies and techniques to teach or provide students with practice learning cognitive skills, such as decision-making. Some CAI program designs include tutorials, 
Integration CAI 144

simulations, virtual reality, drill-and-practice and expert systems (artificial intelligence, or AI). CAI programs are often purchased commercially on a CD-ROM, interactive video disc (IVD), a DVD, a 3.5 disc or floppy disc. In this study, CAI does not refer to Internet-based distance learning where the teacher uses a computer to guide learning and conduct electronic discussions with students.

2. Clinical decision-making is the "operationalization" of nursing knowledge. It is a complex series of decisions nurses make about what to observe, how to intervene and then, depending on the patient's responses, what further actions need to be taken. Clinical decisions are made by nurses with and on-behalf-of-patients for the purpose of attaining the patient's desired goals.

3. Integration strategies entail the skillful planning and management of instruction by the teacher in order to increase the effectiveness and productivity of the course. The foci of the integration strategies are to optimize student learning, reduce the time students and teachers spend on the learning task and reduce course costs in order to gain the most benefit from the large financial investment it takes to purchase and provide CAI as an instructional delivery system.

4. Simulation software programs are abstractions or simplified representations of a system, a situation, or set of processes. Students take on a prescribed role and interact with other participants or elements of the simulated environment. The two types of simulations are experiential and symbolic. In an experiential simulation, the student is placed in the situation and reacts to situations as they emerge. In a symbolic simulation, the student remains "outside" the simulated environment, but exerts control by adding, removing, or 
altering variables.

5. Tutorial software programs present a lesson in basically the same way a teacher in a classroom would present new information. Lessons usually progress from simple-tocomplex and begin by presenting facts and then progress to explaining principles or illustrating a procedure. Many tutorial programs contain a presentation portion and a practice portion in which students are tested for mastery of the content before proceeding to more difficult lessons.

6. Virtual reality (VR) software programs combine computer software and hardware (i.e., head mounted visual display helmet, special gloves, or a mannequin) to create a computer-generated environment that is in someway life-like. Desktop VR simulators integrate clinical decision-making with learning a procedure such as administering cardiopulmonary resuscitation, examining a pregnant client or inserting an intravenous catheter. Learners are able to visualize, manipulate and interact with complex objects portrayed on a computer display screen as they practice their decision-making skills and solve problems. Some types of VR software completely immerse the user in the environment and provide a sense of immediacy and control created by the immersion: the feeling of being there or presence that comes from a changing visual display that is dependent upon eye, or hand, or instrument movements.

7. Expert systems software is an outgrowth of artificial intelligence in which the computer duplicates the decision-making of a human expert in a particular profession. The software is gaining in popularity and is used to assist professionals and novices with decision-making tasks. The software captures and incorporates the knowledge, 
experience, and rules-of-thumb employed by human experts. Depending upon the sophistication of the program, when they are queried, some systems give a diagnosis, call for general and specific knowledge, employ tricks-of-the-trade, include exceptions, reasonable guesses, and reasoning from partial knowledge.

8. Drill-and-practice software is appropriate for educational instruction when rote memorization or practice in basic skills acquisition is the goal such as mathematical skills or drug calculations. The software provides a reliable means for assessing mastery level as scores are kept and students are provided with immediate feedback on their progress. Drill-and-practice software is seldom used to teach higher level cognitive skills, such as decision-making, but data bases of high level multiple-choice questions are available from book publishers and commercial vendors to provide students with practice in testtaking skills.

9. Effectiveness is being able to produce the desired effects, which in this study are increased student learning, reduced costs of the course, reduced teachers' and students' time spent on the learning task.

10. Productivity is the power to accomplish, or bring forth the desired educational goals, which in this study are as follows: Increased student learning, reduced costs of the course, and reduced teachers' and students' time spent on the learning task.

11. Educational productivity is the relationship between the resources expended to provide instruction on one hand, and the outcomes of instruction on the other.

12. The three experts who reviewed the questionnaire for content validity were nurse educators who each have over twenty years of teaching experience and at least ten years 
of experience using CAI to teach clinical decision-making in their educational practices. Each has served on numerous professional evaluation committees and has been involved in the design and evaluation of both course and program evaluation questionnaires.

13. The expert who evaluated the questionnaire for technical and operational reliability has over twenty years experience as a computer consultant who has designed and written several hundred interactive on-line questionnaires and documents for commercial Internet sites. 
Integration of CAI 148

\section{CHAPTER IV}

\section{THE RESULTS}

As stated in Chapter 1, the study reported here examined the problems nurse educators encounter because they lack knowledge of which integration strategies are being used to integrate $\mathrm{CAI}$ into nursing courses and which ones are perceived to be most and least effective for increasing student learning, decreasing costs, as well as the students' and the teachers' time. Nurse educators also lack knowledge about which schools and teachers are perceived to be most effective at integrating CAI, the types of CAI software that are being used to teach clinical decision-making, and what types of software teachers are able to integrate most effectively. The chapter begins with a description of the schools and participants in the sample which is followed by a discussion of the findings of the findings of the five study questions.

\section{The Sample}

- 109 participants from 140 initial contacts (78\% response rate)

- Randomly selected proportionate mix of BSN, AD and diploma schools from each of six geographic regions of the US

- Proportionate mix of $41 \%$ BSN, 54\% AD and 5\% diploma schools closely matched the proportions in the population

- $77 \%$ public and $23 \%$ private schools

- Most public schools were AD (63\%) and most private schools were BSN (60\%)

- Student enrollments range 24 to 999 students (Total 23,196)

- Medium (40\%), small (33\%) and large schools (27\%)

- Majority of the small, medium and large schools were AD schools 


\section{The Sample}

In response to 140 initial e-mail contacts with school administrators or representatives, a total of 109 (a response rate of 78\%) of those who initially agreed to participate in the study completed the questionnaire. As shown in Table 4, the proportionate mix of the types of schools in the sample included 4 more BSN and 4 fewer AD schools than the target number. However, the percent of participating schools were close to the proportionate mix of each type of school in the general population of schools of nursing in the United States. The 5 participants from diploma schools were lower than the intended target number but matched the $5 \%$ proportionate mix of diploma schools in the population exactly (See Table 4).

Table 4. Comparison of Proportionate Mix of Schools in the Population and the Sample $(\mathrm{N}=$ 109)

\begin{tabular}{|c|c|c|}
\hline & Population & Sample \\
\hline \multirow{3}{*}{ Bachelor } & 520 & 45 \\
\hline & $37 \%$ & $41 \%$ \\
\hline & 805 & 59 \\
\hline \multirow[t]{2}{*}{ Associate } & $58 \%$ & $54 \%$ \\
\hline & 74 & 5 \\
\hline \multirow[t]{2}{*}{ Diploma } & $5 \%$ & $5 \%$ \\
\hline & 1,398 & 109 \\
\hline Total & $100 \%$ & $100 \%$ \\
\hline
\end{tabular}


It should be noted that since there were so few diploma schools in the sample, most of the comparisons of the three types of schools where essentially, though not literally, a comparison between the BSN and AD schools. However, after careful consideration, the decision was made to include the findings for the diploma schools because the number in the study did represent the proportionate mix in the general population and as such, was a mirror of what was being done in the population. After reviewing the study in its entirety, the decision to include the diploma schools was supported because some of the findings contributed to the overall quality of the study. The target number of schools for the six geographic regions was closely adhered to during the randomized sample selection process, but since not all participants who agreed to complete the study did so, the sample number fell below the target number for all six regions. The target number for Mid-north Region was inadvertently exceeded by one BSN and one diploma school, but the total number for that region, as well as for the other five geographic regions of the United States, was lower than the intended target number as shown in Table 5. 
Table 5. Comparison of Intended Target and Actual Number of Schools from Each Geographic Region of the United States.

Target Number of Schools from of the Six Geographic Regions

\begin{tabular}{lccccccc}
\hline & MN & MS & NE & NW & SE & SW & Total \\
\hline Bachelor & 9 & 13 & 9 & 8 & 8 & 8 & 51 \\
Associate & 13 & 13 & 13 & 24 & 14 & 14 & 81 \\
Diploma & 2 & 1 & 2 & 1 & 1 & 1 & 8 \\
Total & 24 & 23 & 24 & 23 & 23 & 23 & 140
\end{tabular}

Actual Number of Schools from Each of the Six Geographic Regions in the Sample

\begin{tabular}{lccccccc}
\hline & MN & MS & NE & NW & SE & SW & Total \\
\hline Bachelor & 10 & 7 & 8 & 6 & 5 & 9 & 45 \\
Associate & 9 & 9 & 9 & 12 & 10 & 10 & 59 \\
Diploma & 3 & 0 & 0 & 1 & 1 & 0 & 5 \\
Total & 22 & 16 & 17 & 19 & 16 & 19 & 109
\end{tabular}

\section{Sample Schools}

The sample $(\mathrm{N}=109)$ consisted of $45 \mathrm{BSN}, 59 \mathrm{AD}$ and 5 diploma schools. Public schools made up $77 \%$ of the sample and $23 \%$ were private schools, which meant there were over three times as many public schools as private schools. Most of the public schools were AD schools. The public schools were comprised of 36\% BSN, 63\% AD and 1\% diploma and the private schools were mostly BSN schools. They were made up of $60 \% \mathrm{BSN}, 24 \% \mathrm{AD}$ and $16 \%$ diploma schools. Four of the diploma schools were public and one was private. 
Student enrollments in the school of nursing were widely diverse and ranged anywhere from 24 to 999 students (See Table 6). An analysis of the data showed that there were more students enrolled in the AD schools and that there were four times as many students enrolled in public as in private schools. Even with the high level of school-to-school variability and the fact that there were 14 more AD than BSN schools in the sample, there was only a difference of 1,394 students between the two types of schools (See Table 6). As shown in Table 6, the average student enrollments were higher for BSN schools and were only 31 lower for AD schools but were considerably lower for the diploma schools.

Table 6. Student Enrollments: Number of Students and Average Enrollments in Public and Private Schools $(\mathrm{N}=109)$

\begin{tabular}{lcccccc}
\hline & \multicolumn{3}{c}{ Number of Students } & \multicolumn{5}{c}{ Average Enrollments } \\
\hline & Public & Private & Total & Public & Private & Combined \\
\hline Bachelor $(\mathrm{n}=45)$ & 7,688 & 2,825 & 10,513 & 256 & 188 & 233 \\
Associate $(\mathrm{n}=59)$ & 10,891 & 1,016 & 11,907 & 205 & 169 & 202 \\
Diploma $(\mathrm{n}=5)$ & 100 & 776 & 776 & 100 & 169 & 155 \\
\cline { 2 - 7 } Total $(\mathrm{N}=109)$ & 18,579 & 4,617 & 23,196 & & &
\end{tabular}

For the purposes of comparison and discussion, the schools were divided into arbitrarily designated groups of small, medium and large schools based on the student enrollments in the school of nursing (See Table 7). The small school group was composed of schools with student enrollments that were between 0 through 125 students, the schools in the medium size school group had between 126 through 250 students and the schools in the large school group had 
enrollments between 251 through 999 students. The findings showed that the sample consisted of 33\% small schools, $40 \%$ medium size and 27\% large schools. Associate schools dominated all three groups. One point of interest that was noted was that while the percent of AD and BSN schools in the medium and large groups were almost equal, the $63 \%$ of $\mathrm{AD}$ schools in the small school group were twice the $31 \%$ for the BSN schools.

Table 7. Division of Sample Schools into Three Groups based on Student Enrollments in the School of Nursing $(\mathrm{N}=109)$

\begin{tabular}{|c|c|c|c|}
\hline & Small (24 to 125$)$ & Medium (126 to 250 ) & Large (251 to 999) \\
\hline \multirow{3}{*}{ Bachelor $(n=45)$} & 11 & 21 & 13 \\
\hline & $31 \%$ & $48 \%$ & $45 \%$ \\
\hline & 23 & 22 & 14 \\
\hline \multirow[t]{2}{*}{ Associate $(\mathrm{n}=59)$} & $64 \%$ & $50 \%$ & $48 \%$ \\
\hline & 2 & 1 & 2 \\
\hline \multirow[t]{2}{*}{ Diploma $(\mathrm{n}=5)$} & $6 \%$ & $2 \%$ & $7 \%$ \\
\hline & 36 & 44 & 29 \\
\hline Total $(\mathrm{N}=109)$ & $100 \%$ & $100 \%$ & $100 \%$ \\
\hline Percent & $33 \%$ & $40 \%$ & $27 \%$ \\
\hline
\end{tabular}




\section{CAI-user and Non-user Schools}

- 71\% sample schools used CAI and 29\% did not

- CAI-users AD (62\%), BSN (33\%) and diploma (5\%)

- $56 \%$ of all BSN , $82 \%$ of all $\mathrm{AD}$ and $80 \%$ of diploma CAI-users

- $71 \%$ of all public and $68 \%$ of the private CAI-users

- $63 \%$ of all small, $64 \%$ of all medium and $83 \%$ of all large CAI-users

- More medium size BSN (57\%) were CAI-users than large (45\%) or small (38\%)

- More small AD (83\%) were CAI-users than medium (68\%) or large (24\%)

- Both small, 1 medium and 1 of the 2 large diploma schools were CAI users

\section{CAI-user Schools}

The data collected from the ICAI questionnaire answered the question of the number of schools that used CAI four different times and each answer was different depending on the mix of respondents and the wording of the question. Based on the responses to the first question of the questionnaire, $71 \%$ of the 109 participants indicated they used CAI and $29 \%$ did not. Participants from AD schools indicated their schools used CAI more often than BSN schools and, as expected from the composition of the sample, public schools used CAI more often than private. Overall, large schools used CAI more often than medium or small schools.

More specifically, of the 77 schools that used CAI, $62 \%$ were AD, $33 \%$ were BSN schools and 5\% were diploma schools. Of the 32 non-user schools, $63 \%$ were BSN schools, $34 \%$ were $\mathrm{AD}$ and $3 \%$ were diploma schools (See Table 8 ). 
Table 8. CAI-user Schools and Non-users in the Sample by Type of School: Responses to Question One of the ICAI Questionnaire $(\mathrm{N}=109)$

\begin{tabular}{|c|c|c|c|}
\hline & CAI-users & Non-users & Total \\
\hline \multirow{3}{*}{ Bachelor $(n=45)$} & 25 & 20 & 45 \\
\hline & $33 \%$ & $63 \%$ & $41 \%$ \\
\hline & 48 & 11 & 59 \\
\hline \multirow[t]{2}{*}{ Associate $(n=59)$} & $62 \%$ & $34 \%$ & $54 \%$ \\
\hline & 4 & 1 & 5 \\
\hline \multirow[t]{2}{*}{ Diploma $(n=5)$} & $5 \%$ & $3 \%$ & $5 \%$ \\
\hline & 77 & 32 & 109 \\
\hline Total $(\mathrm{N}=109)$ & $100 \%$ & $100 \%$ & $100 \%$ \\
\hline
\end{tabular}

The same responses were calculated differently in order to compare the percent of all BSN, AD and diploma schools that were CAI-users. The findings showed that only $56 \%$ of all BSN schools used CAI while $82 \%$ of $\mathrm{AD}$ and $80 \%$ of all diploma schools in the sample were CAI-users (See Table 9). 
Table 9. Percent of all Bachelor, Associate and Diploma Schools that Use CAI: Response to Question 1 of the ICAI Questionnaire $(\mathrm{N}=109)$

\begin{tabular}{|c|c|c|c|}
\hline & $\begin{array}{l}\text { Percent of } \\
\text { CAI-users }\end{array}$ & $\begin{array}{l}\text { Percent of } \\
\text { Non-Users }\end{array}$ & Total \\
\hline \multirow{3}{*}{ Bachelor $(n=45)$} & 25 & 20 & 45 \\
\hline & $56 \%$ & $44 \%$ & $100 \%$ \\
\hline & 48 & 11 & 59 \\
\hline \multirow[t]{2}{*}{ Associate $(\mathrm{n}=59)$} & $82 \%$ & $18 \%$ & $100 \%$ \\
\hline & 4 & 1 & 5 \\
\hline \multirow[t]{2}{*}{ Diploma (n - 5) } & $80 \%$ & $20 \%$ & $100 \%$ \\
\hline & 77 & 32 & 109 \\
\hline Total $(\mathrm{N}=109)$ & $71 \%$ & $29 \%$ & $100 \%$ \\
\hline
\end{tabular}


Table 10. CAI-user Schools and Non-user Schools in Sample based on Type of Institution According to Responses to Question 1 of the ICAI Questionnaire $(\mathrm{N}=109)$

\begin{tabular}{|c|c|c|c|}
\hline & CAI-users & Non-Users & Total \\
\hline \multirow{3}{*}{ Public $(\mathrm{n}=84)$} & 60 & 24 & 84 \\
\hline & $71 \%$ & $29 \%$ & $100 \%$ \\
\hline & 17 & 8 & 25 \\
\hline \multirow[t]{2}{*}{ Private $(\mathrm{n}=25)$} & $68 \%$ & $32 \%$ & $100 \%$ \\
\hline & 77 & 32 & 109 \\
\hline Total $(\mathrm{N}=109)$ & $71 \%$ & $29 \%$ & $100 \%$ \\
\hline
\end{tabular}


Table 11. Percent of Schools that are CAI-users and Non-users based on School Size: Responses to Question 1 of the ICAI Questionnaire $(\mathrm{N}=109)$

\begin{tabular}{|c|c|c|c|}
\hline & CAI-users & Non-Users & Total \\
\hline \multirow{3}{*}{ Small $(\mathrm{n}=36)$} & 25 & 11 & 36 \\
\hline & $69 \%$ & $31 \%$ & $100 \%$ \\
\hline & 28 & 16 & 44 \\
\hline \multirow[t]{2}{*}{ Medium $(n=44)$} & $64 \%$ & $36 \%$ & $100 \%$ \\
\hline & 24 & 5 & 29 \\
\hline \multirow[t]{2}{*}{ Large $(\mathrm{n}=29)$} & $83 \%$ & $17 \%$ & $100 \%$ \\
\hline & 77 & 32 & 109 \\
\hline Total $(\mathrm{N}=109)$ & $71 \%$ & $29 \%$ & $100 \%$ \\
\hline
\end{tabular}


Table 12. Percent of CAI-user Schools by Size and Type of School According to Responses to Question 1 of the ICAI Questionnaire $(\mathrm{N}=109)$

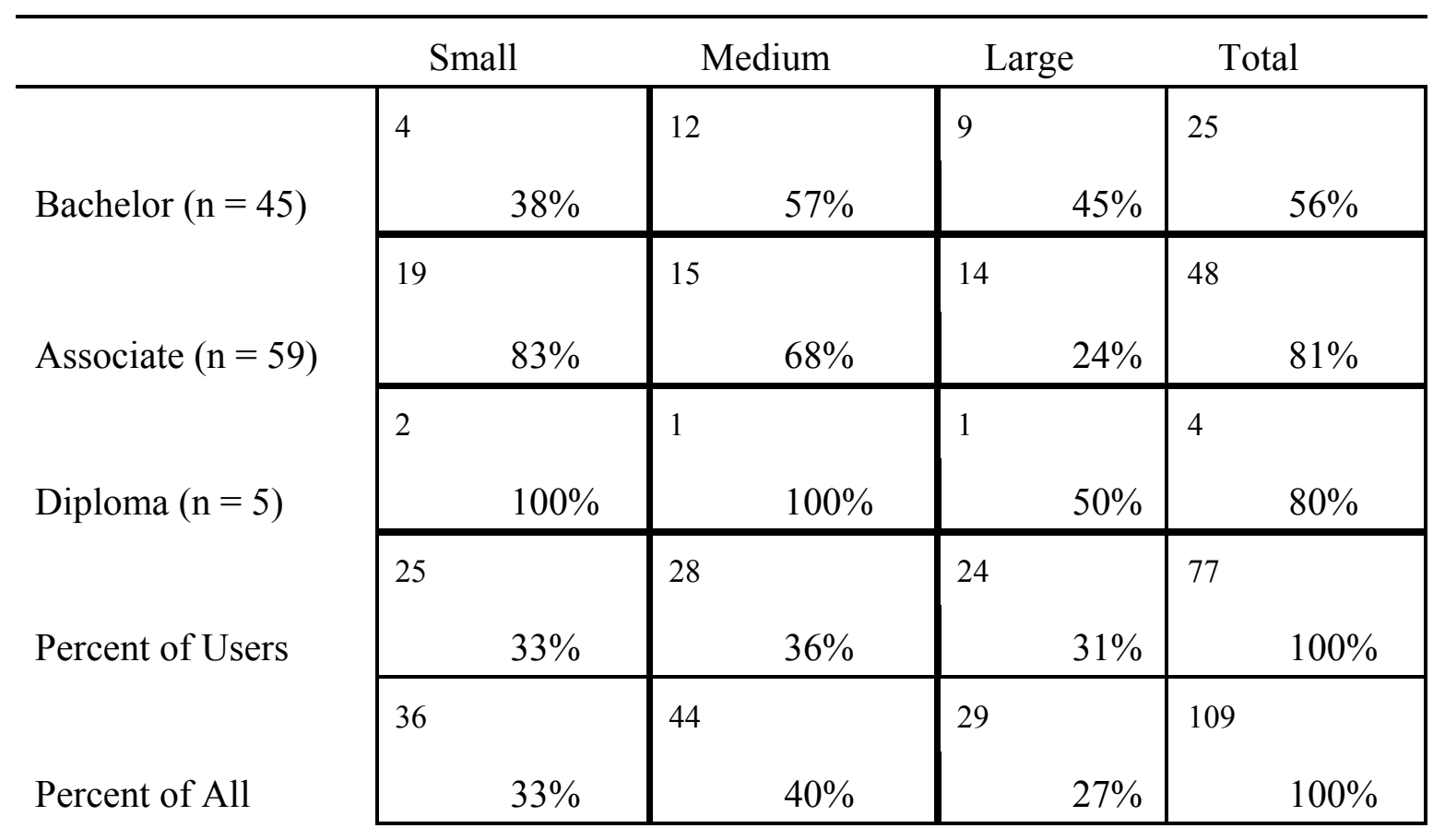


A similar analysis based on the type of institution showed that $71 \%$ of all public schools used CAI and $68 \%$ of all of the private schools did (See Table 10). A third analysis by the size of the schools showed that a higher percent of the large schools used CAI followed by medium and then by small schools (See Table 11). Based on Table 12, a higher percent of medium AD schools were CAI-users than medium BSN schools, but the small AD schools dominated all the others in terms of CAI use. More medium sized BSN schools used CAI followed by large and then small. A much larger percent of small AD schools used CAI followed by the medium schools and then by a much lower percent of the large AD schools. The 2 small diploma schools used CAI as did the single medium but only 1 of the large diploma schools was a CAI-user (See Table 12).

\section{Participants}

- Faculty (89\%), administrators (8\%) computer lab personnel (3\%)

- Females (94\%) and males (6\%)

- 20 to 40 -years (11\%), 41 to 50-years (43\%) and 51-years and older (46\%)

- Masters degree (67\%), Ph. D. (27\%) and BSN or lower $(6 \%)$

\section{Description of the Participants}

Of the 109 participants, $89 \%$ indicated they were faculty members of the school of nursing, $8 \%$ were administrators and 3\% were computer lab personnel. Six percent were males and $94 \%$ were females. Eleven percent indicated they were in the "20 to 40 -year-old" age-group, $43 \%$ were between "41 and 50-year," and 46\% were "over the age of 51 years." Sixty seven percent indicated they had a master's degree, $27 \%$ had a doctorate in nursing or some other field and $6 \%$ had a bachelor's degree or lower. 


\section{A Non-respondent Bias-check}

In an effort to ensure there were not any important differences between those who responded and those who did not, a "non-respondent bias-check" was conducted two months after the deadline for data collection. The data was gathered by sending a bulk mailing of a brief e-mail questionnaire to the group of 32 non-responders. While Smith and Glass (1987) recommend several more direct methods for collecting information from non-responders, such as telephone calls or personal interviews, an e-mail questionnaire was the only avenue available to the researcher because the participant's identities were kept anonymous. The only way non-responders could be identified was through the identification code number that was assigned and distributed at the time of the study. Only 7 of the 32 non-response questionnaires were returned and since the responders did not include their identification code numbers with their answers, it was not possible to send a second non-response questionnaire to those who did not answer the first request.

An analysis of the data disclosed several differences between the sample and nonresponders but, in light of the small number of responders, none of the findings seemed to indicate the non-responders differed in any important ways from the participants. All 7 non-responders indicated they used CAI to teach clinical decision-making as compared to only $71 \%$ of the sample schools, all 7 non-responders were females and a larger percent of the non-responders were administrators than in the sample group. The tables from the non-response bias-check are available in Appendix B1 through B 8 for review by the reader.

The remainder of this chapter will focus on a discussion of the findings of the four study questions beginning with the first question. 
Frequency of Use of Integration Strategies $(n=77)$

Frequently used strategies - 29 of the 44 strategies (66\%)

- Increase Learning 11 of 19 (58\%)

- 4 Preinstruction: "explain CAI, explain objectives, integrate, motivate"

- 3 Mid-instruction: "technical support, instructional support, individual mode"

- 4 Post-instruction: "required, course exams, follow-up activities, postinstruction discussions"

- Decrease Costs 4 of 8 (50\%)

- 3 Mid-instruction: "technical, instructional support, individual mode"

- 1 Post-instruction: "required" instruction

- Decrease the Students' Time 5 of 7 (71\%)

- 2 Preinstruction: "explain CAI, explain objectives"

- 3 Midinstruction: "individual, technical and instructional support"

- Decrease the Teachers' Time 4 of $10(40 \%)$

- 3 Midinstruction: "individual and technical and instructional support"

- 1 Post-instruction: "required"

- Discussion of strategies that were not "frequently" used

- 72\% never "pretested"software and 45\% never "evaluated CAI"

- 92\% use "individualized," 52\% used "small group," 32\% a "whole class"

- $38 \%$ used CAI as an "optional" assignment

- $41 \%$ never used CAI to "replace traditional" teaching

- 28\% used "embedded" test scores as part of course grade

- 47\% never provided an "alternative" method for restudying 


\section{Study Question 1 Findings- Frequency}

The first study question asked: "What integration strategies have nurse educators used to integrate CAI programs designed to teach clinical decision-making into the courses they teach?" As described in Table 3, questions two through six of the ICAI Questionnaire included a list of 19 integration strategies that were compiled from a review of the literature.

The means, standard deviations and medians were calculated to determine how frequently participants used the 19 integration strategies (See Appendix Table C 1). Since the medians and the means were often different and implied different findings, a decision was made to include the medians in the discussion, but to use the means to compare the strategies since they are a more precise measurement. A second decision was made to use a 2.50 mean as the cut-off point to differentiate "frequently" used strategies from the others. The 2.50 mean was selected for several reasons. First, it represented a point on the Likert scale of 1 to 5 at which the strategy was used at least one half of the time. The second was because the 2.50 mean was the point at which $40 \%$ or more of the participants "disagreed" or "strongly disagreed" that the strategy was "frequently" used according to the analysis of the percentages for the individual item response (See Appendix Table C 2). The rationale behind the decision was that, with the exception of the "optional" and "pretest" strategies, the literature recommended all of the strategies be used each and every time CAI was used. Therefore, it seemed reasonable to expect that at least $60 \%$ of the respondents should "agree" that they used the strategy at least once "each semester."

The evaluation of the medians showed that 3 of the strategies were "never" used, 2 were used "monthly" and all of the others were used "each semester." The standard deviations ranged 
from a low of 0.60 for the strategy "explain CAI" to a high of 1.49 for the strategies of "postinstruction discussion" and a "whole-class mode" of instruction.

An analysis of the means showed that there was a total of 29 integration strategies out of a total of 44 that were "frequently" used for all four purposes. Of the four purposes, a higher percent of the strategies were "frequently" used for decreasing the students' time followed by decreasing the teachers' time. In other words, a breakdown of the statistics showed that 11 of the 19 strategies were "frequently" used for increasing student learning, or $58 \%$; a total of 5 of 8 strategies were "frequently" used for decreasing costs, or 63\%; a total of 5 of 7 strategies were "frequently" used for decreasing the students' time, or $71 \%$ and a total of 7 out of 10 for decreasing the teachers' time, or $70 \%$.

The means for the integration strategies that were used to increase student learning showed that four of the five preinstruction strategies had means that were above 2.50 , and 5 of the 5 midinstruction strategies were above and 4 of the 9 post-instruction strategies had means that were above 2.50 (See Table 13). Of the preinstruction strategies for increasing learning, "explain CAI, explain objectives, integrate and motivate" all had means above 2.50 but the fifth strategy of conduct a "pretest" was well below the 2.50 mark. It was noted that the "pretest" strategy was conceptually and operationally closer to the post-instruction strategy of "evaluate CAI," which also had mean below 2.50. The mid-instruction strategies that had the highest means of the entire group were provide "technical" and "instructional support." However, it was noted that both strategies also had relatively high standard deviations as compared with many other strategies with much lower means. The use of an "individual" mode of learning was the third most "frequently" used strategy of the group that also had a mean well above 2.50. Based on their means, an 
"individualized" mode of instruction was used more "frequently" than a "small group" mode which, in turn, was used more often than a "whole class" mode.

For the post-instruction strategies, participants indicated they made CAI a "required" part of the course more often than they designated it as an "optional" assignment, but that they also used CAI to "replace traditional" instruction less than often than the other two strategies. The "required" strategy had a mean that was above 2.50 but the other two strategies, "optional" and "replace traditional" instruction, were both just under the 2.50 mean. The strategy of including CAI content on "course exams" was above 2.50 and was used more often than the other testing strategy of including scores from CAI "embedded" tests. Of the group of three conceptually similar post-instruction strategies, providing "follow-up" activities and "post-instruction" discussions or critiques both had means that were above 2.50 while the strategy of providing "alternative methods" for restudying was below that number.

For the strategies that were used to decrease costs, 4 of the 8 strategies were "frequently" used. They included "individual, technical and instructional support and required." For the strategies that were used to decrease the students' time, 5 of the 7 strategies were "frequently" used. They included "explain objectives, explain CAI, individual, and technical and instructional support." For the strategies that were used to decrease the teachers' time, 4 of the 10 strategies were "frequently" used. They were "individual, technical and instructional support, and required." 
Integration of CAI 166

Table 13. Means of Frequency of Use and Perceived Effectiveness of Integration Strategies for Increasing Student Learning, Decreasing Costs, Students' Time and Teachers' Time $(\mathrm{n}=77)$

\begin{tabular}{lllllll}
\hline Strategy & & Frequency* & Learning** & Costs** & $\begin{array}{l}\text { Students' } \\
\text { Time** }\end{array}$ & $\begin{array}{l}\text { Teachers' } \\
\text { Time ** }\end{array}$ \\
\hline $\mathrm{A}$ & Pretest & 1.64 & 2.46 & & & \\
$\mathrm{~B}$ & Explain Objective & 2.66 & 3.20 & & 2.99 & \\
$\mathrm{C}$ & Explain CAI & 2.92 & 3.34 & & 3.19 & \\
$\mathrm{D}$ & Motivate & 3.05 & 3.32 & & & \\
$\mathrm{E}$ & Integrate & 3.34 & 3.26 & & & \\
\hline $\mathrm{F}$ & Individual & 3.56 & 3.28 & 2.69 & 2.88 & 3.00 \\
$\mathrm{G}$ & Small Group & 2.42 & 2.83 & 2.39 & 2.45 & 2.61 \\
$\mathrm{H}$ & Whole Class & 2.17 & 2.72 & 2.28 & 2.55 & 2.45 \\
$\mathrm{I}$ & Technical Support & 3.70 & 3.58 & 2.56 & 3.39 & 3.20 \\
$\mathrm{~J}$ & Instructional Support & 3.78 & 3.59 & 2.57 & 3.39 & 3.05 \\
\hline $\mathrm{K}$ & Post Instruction & 2.74 & 3.13 & & & \\
$\mathrm{~L}$ & Follow-up & 2.96 & 3.08 & & & \\
$\mathrm{M}$ & Required & 2.99 & 3.22 & 2.55 & & \\
$\mathrm{~N}$ & Optional & 2.43 & 2.75 & 2.32 & & 2.70 \\
$\mathrm{O}$ & Course Exam & 2.88 & 3.15 & & & 2.47 \\
$\mathrm{P}$ & Embedded & 1.62 & 2.47 & & & 2.59 \\
$\mathrm{Q}$ & Replace Traditional & 2.35 & 2.77 & 2.61 & & 2.39 \\
$\mathrm{R}$ & Alternative & 2.21 & 2.92 & & & 2.82 \\
$\mathrm{~S}$ & Evaluate CAI & 2.18 & 2.99 & & &
\end{tabular}

** Likert Scale 1 to 4

* Likert Scale 1 to 5 
An item-by-item analysis of the percentages of the frequency of use permitted a closer examination of the participants' responses to individual items (See Appendix Table C 2). In particular, the percentages shed light on the reason the standard deviations for the strategies of providing "technical" and "instructional support" were higher than strategies such as "explain CAI" and "explain objectives" that had much lower means. As Appendix Table C 2 shows, the percentages for the strategies "explain the CAI" and "explain the objectives" were $72 \%$ and $83 \%$ respectively for the " $3=$ Each Semester" response but both had extremely low percentages for each of the other four response options which meant the standard deviations were very low for both strategies. In contrast, the percentages for the strategies of provide "technical" and "instructional support" were roughly divided in half between the " $5=$ Weekly" and the " $3=$ Each Semester" options. Since more participants selected the " $5=$ Weekly" response, the means were higher than for the "explain CAI" and "explain the objectives" strategies, but because the responses were split across three response options (each semester, monthly, and weekly), the variances for the responses were greater as reflected by the higher standard deviations. Also, as will become apparent later in the chapter, the correlations between the frequency of use and the measures of perceived effectiveness were extremely low for the strategies of providing "technical" and provide "instructional support"in almost every instance throughout the study. The low correlations simply reflect the small common variance between the frequency of use and perceived effectiveness for the strategies even though the means for the strategies topped almost every list.

An examination of the percentages also shed further light on the two strategies of "pretest" and "evaluate CAI." As mentioned previously, both had very low means which, if observed in isolation, seemed to indicate educators seldom evaluated the CAI they used either before or after it 
was used. However, as shown in Appendix Table C 2,72\% of the participants indicated they "never" conducted a pretest and only $18 \%$ conducted a pretest at least once a "semester" and while $42 \%$ indicated they made it a practice to "never" evaluate CAI, another $45 \%$ did evaluate the software at least "once a semester." It seems almost half of the participants conducted some type of evaluation of the CAI at least once a semester. Based on the percentages for the three modes of learning, $92 \%$ of the participants indicated they used an "individualized mode" of instruction at least once a semester while 52\% indicated they used "a small-group mode," and only $34 \%$ used CAI for "whole-class learning" each semester. Also, $51 \%$ of the participants indicated they designated CAI as a "required" assignment and 38\% designated it as an "optional"assignment at least "each semester." Also, while 35\% used CAI to "replace traditional" teaching methods each semester, $41 \%$ never used it that way.

In summary, the first study question looked at the frequency of use of the integration strategies. Based on the medians, three strategies were "never" used, two were used "monthly" and all of the others were used "each semester." The means showed that a 29 of the 44 strategies, or $66 \%$ were "frequently" used for all four purposes. The group used a higher percent of the strategies for decreasing the students' time followed by decreasing the teachers' time.

For the strategies to increase student learning, only 11 of the 19 strategies, or $58 \%$ were "frequently" used. Four of the 5 preinstruction strategies, 3 of the 5 midinstruction strategies and 4 of the 9 postinstruction strategies were "frequently" used. The four conceptually similar preinstruction strategies were "frequently" used but that the fifth strategy, "pretest," fell far below. The two strategies with the highest means were providing "technical" and "instructional support." An "individual" mode of instruction had the third highest mean of the group and was used more 
often than the other two modes of instruction of "small group" and "whole class" instruction. Designating CAI as a "required" assignment was "frequently" used and used more often than making it an "optional" assignment but both strategies were used more often than using it to "replace traditional" instruction. Two of the three conceptually similar post-instruction strategies had means that were above 2.50 but provide "alternative" ways of restudying was below the 2.50 level. Participants indicated they tested the CAI content as part of "course exams" much more often than they used the scores from "embedded tests."

Four of the 8 , or $63 \%$ of the strategies to decrease costs were used "frequently", 5 of the 7 to decrease the students' time, or $71 \%$, and 4 of the 10 strategies to decrease the teachers' time, or $40 \%$, were "frequently" used. 
Effectiveness of Integration Strategies $(\mathrm{n}=77)$

- 17 of 44 strategies perceived "effective" (39\%)

- Increase Learning 11 of 19 (58\%)

- The exact same 11 strategies (58\%) that were "frequently" used were perceived to be "effective" for increasing student learning

- "Instructional" and "technical support" had the highest means for "frequency" of use and "effectiveness"

- "Individual" mode of instruction was "effective" but "small group" and "whole class" modes were not

- "Optional" assignment was not "effective" but "required" was

- "Replace traditional" not "effective" for increasing student learning

- "Post-instruction" discussion or critique and "follow-up" activities "effective"

- "Alternative" method of restudying was not "effective"

- "Embedded" tests was not "effective" but including CAI content on a "course exam" was an "effective" strategy

\section{Study Question 2 Findings- Effectiveness}

The second study question asked: "How effective do nurse educators perceive the integration strategies have been for increased student learning, decreased costs, teachers' time and students' time?" It should be noted that the Likert scale that was used for Questions three, four, five and six of the questionnaire was based on a scale of 1 "Strongly disagree" to 4 "Strongly agree," rather than the 1 to 5 Likert scale that measure time spans was used for the second question. A mean of 3.00 was the number that was designated as the cut-off point for determining if a strategy was perceived by participants as "effective." A means of 3.00 was selected as a cut-off 
point because it correlated with the "agreed" option on the Likert scale which indicated that the participant perceived the strategy was "effective," but it also indicated that at least $85 \%$ of the participants" either "agreed" or "strongly agreed" that the strategy was "effective"on the individual item-by-item analysis.

\section{Strategies to Increase Learning}

The means, standard deviations and medians of the effectiveness of the strategies for increasing student learning were calculated (See Appendix Table C 3). An examination of the medians showed that most participants "strongly agreed" that the strategies of provide "technical" and "instructional support" and a majority "agreed" that the other 17 strategies were "effective" for integrating CAI to increase student learning. The standard deviations ranged from a low of 0.59 for "provide instructional support" to a high of 0.94 for the strategies of "pretest" and use "embedded" test scores as part of the course grade. The standard deviations were generally lower and the means higher for all but three strategies than the findings for the frequency of use.

In general, the means and patterns of responses indicated that participants used the strategies they perceived as most "effective"for increasing student learning most often and did not use the strategies that were not perceived to be "effective"frequently. Of the 19 strategies, participants rated 11 , or $58 \%$ of the 19 strategies as "effective." The means for 4 of the 5 preinstruction strategies, 3 of the mid-instruction strategies and 4 of the post-instruction strategies were above 3.00. In other words, the exactly same strategies that were above 2.50 mean for the frequency of use were also above the 3.00 mean for perceived "effectiveness" of the strategies for increasing learning. 
The 4 conceptually similar preinstruction strategies fell into a different pattern when ranked by their means than for the frequency of use but they were all above the 3.00 cut-off point and were, once again, far above the mean for the "pretest" strategy. Also, as with the means for the frequency of use, the post-instruction strategy of "evaluate CAI" was far below the 3.00 mean cutoff point. As mentioned previously, the strategies of provide "instructional" and "technical" support had the highest means for both "frequency" and "effectiveness." The third highest mean was for the use of an "individual" mode of instruction which was rated above 3.00 and far above the "small group" and "whole class" modes of instruction, both of which were well below 3.00. The strategy of designating CAI as a "required" assignment was above 3.00 while using CAI as an "optional" assignment and using CAI to "replace traditional" instruction were both below. Once again, the strategies of holding a "post-instruction" discussion or critique and the use of "followup" activities were both above 3.00 while the remedial strategy of providing an "alternative" method of restudying the material was below. The strategy of including CAI content on the "course exam" was well above 3.00 but the other testing strategy of the use of "embedded" tests was perceived to be the least "effective" strategy of the group for increasing student learning.

The analysis of the percentages of the perceived "effectiveness" of the integration strategies for increasing student learning is available for review in Appendix Table C 4. Some of the findings that were of particular interest showed that $37 \%$ either "disagreed" or "strongly disagreed" that the strategy of "pretest" learners to ensure they are adequately prepared to learn the content of a CAI program was "effective"for increasing student learning. Another was that $49 \%$ either "disagreed" or "strongly disagreed" that the use of the scores from CAI "embedded" tests was an "effective"strategy for increasing student learning. 
Effectiveness of the Integration Strategies for Decreasing Costs

- 0 of $8(0 \%)$ were perceived to be effective for decreasing costs

- "Individual" mode highest mean

- $61 \%$ agreed "replace traditional" reduced costs but mean was below 3.00

\section{Strategies to Decrease Costs}

The measures of central tendencies were analyzed to determine the participants' perceptions of the "effectiveness" of the integration strategies for decreasing course costs (See Appendix Table C 5). An evaluation of the median scores indicated that participants perceived that one half of the strategies were "effective"for reducing costs. The "effective"strategies included the use of "individualized" and a "small-group mode," designating CAI as a "required" assignment and using it to "replace traditional" instruction. Those that were not perceived to be "effective" cost-reducers included a "whole class" mode, providing "technical" and "instructional support," and designating the CAI assignment as an "optional" assignment. The standard deviations were relatively small as compared with "effectiveness" for increasing learning and ranged from a low of 0.71 for the strategy of designate the CAI assignment as "optional" to highs of 0.97 and 0.98 for provide "technical" and "instructional support."

The means (See Table 13) were generally lower than the means for student learning and were clustered together with a low range of between 2.20 to 2.60 . There were no strategies with a mean above 3.00, which meant that the participants did not perceive any of the strategies were "effective" for reducing costs. The strategies with the highest means were "individual mode" of instruction and using CAI to "replace traditional" instruction. 
An analysis of the percentages for the individual items (See Appendix Table C 6) showed some interesting findings. For instance, $61 \%$ either "agreed" or "strongly agreed" that using CAI to "replace traditional" instruction reduced course costs but the mean was below 3.00. Another related finding showed that while $45 \%$ of the participants either "disagreed" or "strongly disagreed" that designating CAI as a "required" assignment reduced costs, 62\% either "disagreed"or "strongly disagreed" that designating it as an "optional" assignment did.

Effectiveness of the Integration Strategies for Decreasing the Students' Time

- 3 of 7 were "effective" (43\%)

- "Explain CAI" "effective" but "explain objectives" just under 3.00

- 95\% "technical" and "instructional" effective

Strategies for Decreasing the Students' Time

An analysis of the means, standard deviations and medians was conducted to determine how "effective" the participants perceived the integration strategies were for reducing students' time to learn (See Appendix Table C 7).

Based on the median scores, all of the strategies, except the use of a "small group" mode of instruction, were perceived to be "effective" for reducing the students' time. The standard deviations were relatively small and ranged from a low of 0.63 for provide "technical" support to a high of 0.82 for the use of a "whole class" mode.

According to the means, (See Table 13), there was 1 preinstruction strategy above 3.00 and 2 mid-instruction strategies that were above. The preinstruction strategy was "explain CAI" and the mid-instruction strategies were provide "technical" and "instructional" support. The other 
preinstruction strategy, "explain the objectives" was close but below 3.00 and all three modes of instruction were below 3.00. The "individual mode" was perceived to be more "effective"than "whole class," which was followed by a "small group" mode.

The percentages for the individual items were calculated (See Appendix Table C 8) and 1of the findings of interest showed that the group was divided in half about whether or not a "small group" or a "whole class" mode of instruction was an "effective" strategy for decreasing the students' time. In contrast, $73 \%$ "agreed" that an "individual" mode of instruction saved the students' time. The percentages for "instructional" and "technical" support showed that 95\% perceived the strategies saved the students' time.

Summary of Effectiveness of Integration Strategies for Decreasing Teachers' Time

- 3 of 10 "effective" (30\%)

- "Technical" and "instructional" support were "effective"

- "Individual" only "effective" mode

- "Small group" higher than "whole class"

- 73\% using CAI to "replace traditional" decreased time but not "effective"

- 65\% agreed "required" saved teachers' time but not "effective"

- 46\% thought "optional" saved the teachers' time

- Scores from "embedded" tests had the lowest mean of all the strategies

\section{Strategies to Decrease the Teachers' Time}

The measures of central tendencies were calculated to determine the participant's perceptions of the effectiveness of the integration strategies for decreasing the teachers' time (See Appendix Table C 9). 
The median scores showed that participants "disagreed" that a "whole class" mode of instruction and the strategy of including "embedded" tests scores as part of the course grade saved the teachers' time. Participants "agreed" that 6 of 9 of the strategies and "strongly agreed" that the strategy providing "technical" support was "effective"for saving the teachers' time. The standard deviations ranged from a low of 0.77 to a high of 1.01 for the strategies of "instructional support," "required," and "course-exam."

An examination of the means (See Table 13) showed that 3 of 10 , or $30 \%$ of the strategies were rated as "effective"by participants. More precisely, 3 of the 5 mid-instruction strategies and none of the 5 post-instruction strategies were rated as "effective" for saving the teachers' time. The mid-instruction strategies of provide "technical" and "instructional support" were perceived to be "effective" for decreasing the teachers' time and the mean for an "individual" mode of instruction was above 3.00. As before, the "individual mode" was rated higher than a "small group" mode, which was, in turn, higher than the use of "whole class" mode. The use of CAI to "replace traditional" instruction was below 3.00 but was rated slightly higher than making it a "required" assignment, which was rated higher than making it an "optional" assignment. The strategy that was perceived to be least "effective"for decreasing the teachers' time was including scores from "embedded tests," which was lower than including test questions on "course exams."

Again, the percentages of the participants' responses to individual items were calculated (See Appendix Table C 10). Some of the more interesting findings included the fact that $73 \%$ either "agree," or "strongly agree" that using CAI to "replace traditional" instruction saved the teachers" time. Another showed that $46 \%$ of the participants either "agreed"or "strongly agreed" 
that designating CAI as an "optional" assignment saved the teachers' time while $65 \%$ either "agreed" or "strongly agreed" that making it a "requirement" did.

In summary, an evaluation of the second study question findings showed that $58 \%$ of strategies to increase learning, none of the strategies to decrease costs, $43 \%$ to decrease the students' time and only $30 \%$ of the strategies to decrease the teachers' time were perceived to be "effective"for those purposes by the participants. It was noted that there was perfect agreement between the 11 strategies that were "frequently" used and those that were "effective" for student learning. However, of the 4 strategies that were "frequently" used for reducing costs, none were perceived to be "effective"for that purpose. While participants indicated they "frequently" used 5 strategies for decreasing the students" time, only "technical" and "instructional support" and "explain CAI" were perceived of as "effective" for that purpose. Participants also used 5 strategies for decreasing the teachers' time "frequently", but only the strategies of providing "technical" and "instructional support" and using an "individual" mode of instruction were perceived of as "effective" for decreasing the teachers' time. 
Correlations Between Frequency of Use and Effective Strategies

- Student Learning- 15 significant

- 7 weak but significant "explain CAI, explain objectives, motivate, integrate, individual mode, course exam and follow-up"

- "Technical" and "instructional" support not significant but highest means for both "frequency" and "effectiveness"

- 8 strongest correlations did not meet other two criteria

- Decrease Costs: 2 significant "replace traditional" and "whole class" neither met other criteria

- Decrease Students' Time: 2 significant "small group" and "whole class" but neither met other criteria

- Decrease Teachers' Time: 1 significant correlation "optional" but did not meet other criteria

\section{Correlation Findings}

A Pearson r Product Moment Correlation Coefficient was calculated to determine the relationships between the frequency of use and perceived effectiveness of the strategies for integrating CAI into courses. The Pearson $r$ was selected because it is the most appropriate test when both variables to be correlated are expressed as interval data (Gay, 1992).

The results (See Table 14) showed 15 of the 19 were statistically significant relationships for increased student learning, 2 of the 8 strategies for decreased costs, 2 of the 7 strategies for decreasing the students' time and 1 of the 10 strategies for decreasing the teachers' time (at a 0.01 level of significance). The strongest correlations were the strategies that were not "frequently" used (mean < 2.50) and were not perceived to be "effective" for increasing student learning (mean $<3.00)$. Ranked from the highest to the lowest based on the means for perceived 
effectiveness, they included, "evaluate CAI, alternative, small group, replace traditional, optional, whole class, pretest, and embedded testing." Many of the strategies that were used "frequently" used and perceived to be "effective"for increasing student learning were also statistically significant, but the correlations were weaker. Ranked from the highest to the lowest based on the means for effectiveness they were "explain CAI, explain objectives, motivate, integrate, individual mode, course exam, and follow-up." As mentioned previously, the strategies of provide "technical" and "instructional support"consistently had the highest means for frequency of use and perceived effectiveness and yet, due to a small common variance, had extremely weak correlation scores. 
Table 14. Correlations Between Frequency of Use and Perceived Effectiveness of the Integration Strategies $(\mathrm{n}=77)$

\begin{tabular}{|c|c|c|c|c|c|}
\hline Strategy & & Learning & Costs & $\begin{array}{c}\text { Students' } \\
\text { Time }\end{array}$ & $\begin{array}{c}\text { Teachers' } \\
\text { Time }\end{array}$ \\
\hline A & Pretest & $0.51^{*}$ & & & \\
\hline B & Explain Objectives & $0.38^{*}$ & & 0.26 & \\
\hline $\mathrm{C}$ & Explain CAI & $0.30^{*}$ & & 0.27 & \\
\hline $\mathrm{D}$ & Motivate & $0.34 *$ & & & \\
\hline E & Integrate & $0.46^{*}$ & & & \\
\hline $\mathrm{F}$ & Individual & $0.32 *$ & 0.17 & 0.09 & -0.11 \\
\hline G & Small Group & $0.45^{*}$ & 0.18 & $0.35^{*}$ & 0.16 \\
\hline $\mathrm{H}$ & Whole-class & $0.60 *$ & $0.37^{*}$ & $0.30^{*}$ & 0.17 \\
\hline I & Technical Support & 0.09 & -0.02 & -0.09 & 0.03 \\
\hline $\mathrm{J}$ & Instructional Support & 0.26 & 0.06 & 0.05 & 0.05 \\
\hline K & Post-instruction & 0.20 & & & \\
\hline $\mathrm{L}$ & Follow-up & $0.36^{*}$ & & & \\
\hline M & Required & 0.29 & 0.29 & & 0.05 \\
\hline $\mathrm{N}$ & Optional & $0.56^{*}$ & 0.11 & & $0.42 *$ \\
\hline $\mathrm{O}$ & Course Exam & $0.49^{*}$ & & & 0.13 \\
\hline$P$ & Embedded & $0.52 *$ & & & 0.18 \\
\hline Q & Replace Traditional & $0.53 *$ & $0.39 *$ & & 0.20 \\
\hline $\mathrm{R}$ & Alternative & $0.48^{*}$ & & & \\
\hline S & Evaluate CAI & $0.36^{*}$ & & & \\
\hline
\end{tabular}


There were only two statistically significant correlations among the strategies for decreasing costs. The first, "replace traditional" instruction had a low mean for "frequency" of use but was next to the highest for perceived "effectiveness." The other, "whole class" mode of instruction was rated as least used and least "effective." The strategies of "small group"and "whole class" mode were the two statistically significant correlations for decreasing students' time. Both had the lowest means for "frequency" of use and perceived "effectiveness." There was also a fairly strong significant correlation for the strategy of designating CAI as "optional" to decrease the teachers' time. However, once again, the strategy was seldom used and not perceived to be "effective" for that purpose.

In summary, the correlations between the frequency of use and effectiveness of the strategies showed that 15 of the 19 strategies were statistically significant for increasing student learning, 2 of the 8 strategies for decreasing costs, 2 of the 7 strategies for decreasing the students' time and 1 of the 10 strategies for decreasing the teachers' time. The strongest relationships were between the 8 strategies that were not "frequently" used or perceived to be "effective." There were 7 other strategies that were "frequently" used and were perceived to be "effective" for increasing student learning that had weaker, but statistically significant correlations. The strategies of providing "technical" and "instructional support" were the two most "frequently" used and perceived to be the most "effective" strategies for student learning, decreasing the students' time and the teachers' time but, as explained before, they had low common variances and extremely low correlations. 


\section{Study Question 3 Finding- Key Features of Schools}

The third study question asked, "What are the key features of the schools and educators that are perceived by participants to integrate CAI designed to teach clinical decision-making effectively?" The focus of this section was on identifying the "key features" of the schools that were perceived by participants to be "effective"at integrating CAI. The discussion began by looking at the frequency of use of the integration strategies to determine if there are differences in how often the different types of schools used the strategies or how "effective"they perceive the strategies were for each of the four purposes. Next, the discussion focused on the computer resources that were available for students to use at the different types of schools such as a computer lab, computers, locations of computers, software, support personnel, and the number and percent of teachers who use CAI. 
Comparison of the Frequency of Use of the Integration Strategies by Type of School

- BSN 36 (81\%), AD 28 (63\%) diploma 22 (50\%) of 44

- Increase Student Learning:

- BSN used 14 (73\%), AD used 12 (63\%) and diploma used 9 (47\%) of 19

- Decrease costs

- BSN used 7 (88\%) and AD and diploma used 5 of 8 (63\%)

- AD and diploma used "optional" but BSN used CAI to "replace"

- Decrease Students' Time

- BSN used 7 (100\%), AD used 5 (71\%) and diploma used 3 (43\%) of 7

- BSN used "small group" and "whole class"

- Decrease Teachers' Time

- BSN used 8 of $10(80 \%)$, AD used $6(60 \%)$ and diploma used $5(50 \%)$

- BSN and AD used "course exams," but none used "embedded" test

\section{Comparison of the Frequency of Use}

The following discussion provides a brief overview of the comparison of the "frequency" of use of the integration strategies by the three types of schools. In light of the extremely small sample size for the diploma schools $(\mathrm{n}=4)$, the decision was made to include the findings in this part of the discussion simply because the number of participants in the group so closely represented the percent in the general population but the findings were not given a great deal of credence since the number of representatives of the group was so small.

The means for the frequency of use of the strategies were compared and the findings showed that BSN schools used 36 , or $81 \%$, AD schools used 28 , or $63 \%$, and diploma schools used 
22 , or $50 \%$ of the strategies "frequently." The largest differences between BSN and AD schools were in the "frequency" of use of the strategies to decrease the students' time, costs and the teachers' time. In other words, there was more agreement between the schools on the strategies to increase student learning than on the other three purposes. For instance, BSN schools used 14 of the 19 strategies, or $73 \%$ of the strategies to increase student learning while AD schools used 12 , or $63 \%$. The diploma schools only used 9 , or $47 \%$ of the strategies "frequently." For the strategies to decrease costs, BSN schools used 7, or $88 \%$, while AD and diploma schools both used 5, or $63 \%$ "frequently." For the strategies to decrease the students' time, BSN schools used all 7, or 100\%, while AD schools used 5, or 71\%, and diploma schools used 3, or $43 \%$ of the strategies "frequently." For the strategies to decrease the teachers' time, BSN schools used 8 of the 10 strategies, or $80 \%$, AD schools used 6 , or $60 \%$ and diploma schools used 5 , or $50 \%$ of the strategies "frequently." When the BSN and AD schools were compared, there was only a $10 \%$ difference for student learning but there was a $25 \%$ difference for costs, a $29 \%$ difference for strategies to decrease students' time and a $20 \%$ difference for the teachers' time. A comparison of the BSN and diploma school's responses showed the largest difference was $57 \%$ for the strategies to decrease the student's time followed by a $30 \%$ difference for strategies to decrease the teachers' time, a $26 \%$ difference for student learning and $25 \%$ difference for strategies to decrease costs.

While the patterns of use of the strategies were similar for BSN and AD schools, BSN schools used several strategies "frequently" that matched the recommendations of the experts in the literature for increasing educational productivity that $\mathrm{AD}$ and diploma schools did not (See Appendix Table C 11). Also, AD and diploma schools used the strategy of assigning CAI as an “optional" assignment for three purposes (student learning, costs and teachers' time), which was 
not recommended as an effective way to increase educational productivity in the literature (Bates, 1999). The findings for the diploma schools showed that the means for most of the strategies were far below those for the BSN or AD schools and the strategies they used "frequently" were very different than the other two.

For the strategies to increase student learning, the findings showed that BSN and AD schools "frequently" used four of the five preinstruction strategies of "explain CAI, explain objectives, integrate and motivate" while the diploma schools only used two preinstruction strategies "frequently". The strategies of "instructional" and "technical support"and an "individual"mode of instruction were "frequently" used by all three types of schools. A "small group" and "whole class" mode of instruction were "frequently" used by BSN schools but were almost never used by diploma schools. Both BSN and AD schools used the five post-instruction strategies of "follow-up, required, a post-instruction discussion and course exam" "frequently." Diploma schools only used three post-instruction strategies "frequently," which included "required, alternative and post-instruction." The same data was analyzed to compare the strategies that were used to decrease costs and the findings showed that BSN schools used 7, or $88 \%$ of the strategies "frequently" while AD and diploma schools each used 5, or $63 \%$ of the strategies "frequently." As mentioned above, BSN schools were the only ones to use CAI to "replace traditional" instruction "frequently" while AD and diploma schools reported using the "optional" strategy "frequently". When the data was analyzed to determine how "frequently" the different types of schools used the strategies that were intended to decrease the students' time, the findings showed that BSN schools used all 7 of the strategies "frequently," AD schools used 5, or 71\% and diploma schools used 3, or $43 \%$ of the strategies "frequently." Of particular interest was the finding that only BSN schools 
used the "small group" and "whole class" modes of instruction "frequently." When the data was analyzed for the strategies to decrease the teachers' time, the findings showed that BSN schools used 8 of the 10 strategies, or $80 \%$, AD schools used 6 , or $60 \%$ and diploma schools used 5 , or $50 \%$ of the strategies "frequently." While BSN and AD schools both used the strategies of including CAI content on in-class "course exams," none of the schools used a major teacher time-saving strategy of using scores from "embedded" tests "frequently." As mentioned previously, BSN school participants were the only ones to use CAI to "replace traditional" instruction "frequently." 


\section{Comparison of Frequency of Use of Integration Strategies by Type of Institution}

- Public used 29 (66\%) and private used 23 (52\%) of 44

- Increase Student Learning

- Public used 12 (63\%) and private used $10(53 \%)$ of 19

- Decrease Costs

- Public used $5(63 \%)$ and private used $4(50 \%)$ of 8

- Decrease Students' Time

- Public used $6(86 \%)$ and private used 5 (71\%) of 7

- Decrease Teachers' Time

- Public used $6(60 \%)$ and private used $5(50 \%)$ of 10

- Public used "small group" and "post-instruction"

An analysis was conducted to compare the means of the two types of institutions (See Appendix Table C 12) and the findings showed that, for the most part, public schools used the strategies more "frequently" than private schools but the patterns of used were similar. Of the 44 strategies, public schools used 29 , or $66 \%$ and private schools used 23 , or $52 \%$ of the strategies "frequently." Of the strategies that were intended to increase student learning, public schools used 12 , or $63 \%$ "frequently" and private schools used 10 of 19 , or $53 \%$. The same 4 preinstruction strategies were "frequently" used by both public and private schools but the means for the "pretest"strategy indicated private schools almost never used the strategy. Of the 5 mid-instruction strategies, public schools used 4 and private schools used 3 "frequently." Only public schools used the "small group"mode of instruction "frequently." Of the 9 post-instruction strategies, public schools used 4 "frequently" and private schools used 3. The strategies that were "frequently" used 
by both included "follow-up, required, course exam" and only public schools used the "postinstruction" discussion or critique strategy.

Analysis of the "frequency" of use of the strategies to decrease costs showed that private schools used 4 of the strategies "frequently" and public schools used 5. Only public schools used the "small group" strategy. For the strategies to decrease the students' time, public schools used 6 strategies "frequently" and private schools used 5. Public schools used the "whole class" strategy and private schools did not. For the strategies to decrease the teachers' time, public schools used 6 strategies "frequently" and private schools only used 5.

Comparison of Frequency of Use of Integration Strategies by School Size

- Small used 19 (43\%), medium used 34 (77\%) and large used 32 (73\%) of the 44

- Increase Student Learning

- Small used 7 (36\%), medium and large used 13 (68\%)

- Small schools only used 1 post-instruction strategy "required" medium and large used 5 others

- Decrease Costs

- Small used 4 (50\%), medium used 7 (88\%) and large used $6(75 \%)$ of 8

- Decrease Students' Time

- Small used 4 (57\%), medium and large used 6 (86\%) of 7

- Decrease Teachers' Time

- Small used 4 (40\%), medium used 8 (80\%) and large schools used $7(70 \%)$ of the 10 strategies "frequently"

A third analysis was conducted to compare the means of the three different sizes of schools to see how "frequently" they used the strategies (See Appendix Table C 13). The findings showed 
that small schools used far fewer strategies "frequently" than the medium and large schools. Stated differently, small schools used 19 , or $43 \%$ of the 44 strategies, medium size schools used 34 , or $77 \%$ and large schools used 32 , or $73 \%$ of the strategies "frequently."

For the strategies to increase student learning, small schools used 7 , or $36 \%$ of the strategies and medium and large schools both used 14 , or $68 \%$ of the 19 strategies "frequently." According to the means, all three groups used the 4 preinstruction strategies "frequently" with the exception of the "explain objectives" strategy which was not "frequently" used by the small schools and none of the schools used the "pretest" strategy "frequently." For the mid-instruction strategies, all three sizes of schools used "technical" and "instructional" support and an "individualized" mode of instruction, but only medium and large schools used the "small group" strategy "frequently." For the post-instruction strategies, the small schools only used the "required" strategy "frequently" but medium and large schools used 6 of the 9 post-instruction strategies "frequently." Both used the "follow-up, required, replace traditional, post-instruction, and course exam" strategies "frequently." Medium size schools also used the "optional" strategy "frequently" and large schools used "evaluate CAI" "frequently."

The analysis of the findings for the integration strategies to decrease costs showed that small schools used 4 , or $50 \%$ while medium schools used 7 , or $88 \%$ and large schools used 6 , or $75 \%$ of the 8 strategies "frequently." The differences between the groups were that small schools did not use the "optional, small-group or replace traditional" instruction strategies but the medium size schools used the strategies for several different purposes. The difference between the medium and large schools was that medium schools used the "optional" strategy for three different purposes and large schools did not. The analysis for the strategies to decrease the students' time showed that 
small schools used 4 , or $57 \%$ and medium and large schools each used 6 , or $86 \%$ of the strategies "frequently." The differences between the schools were that small schools did not use the "explain objectives" strategy "frequently" and the others did. The analysis of the strategies to decrease the teacher's time showed that small schools used 4 , or $40 \%$ of the strategies, medium schools used 8 , or $80 \%$ and large schools used 7 , or $70 \%$ of the 10 strategies "frequently." The differences between the schools were that small schools did not use the "course exam, optional, small-group, or replace traditional"strategies "frequently" but the medium size schools did. The difference between the medium and large schools was, again, that large schools did not use the "optional" strategy "frequently" while the medium size schools did.

In summary, the comparison of the three types of schools showed that BSN schools used more strategies "frequently" than either AD or diploma schools. The sparse data available from the diploma schools indicated that they used all of the strategies less than the others and that they used different strategies. A comparison of public and private schools showed that public schools used almost all of the strategies slightly more often than private schools but that the patterns of use were similar. The comparison of the three different size schools showed that medium and large schools used many more strategies "frequently" than the small schools. The patterns of use were similar among the three sizes of schools but there were a number of strategies small schools did not use "frequently" that the others did. None of the strategies that were not used were related to the resources of the schools such as a failure to provide technical and instructional support due to financial considerations. 
Next, the discussion focused on the findings to compare how participants from different schools perceived the "effectiveness" of the strategies for increasing learning, reducing costs, decreasing the students' time and the teachers' time.

Comparison of Effectiveness of Strategies for Increasing Student Learning by Type of School

- Differences between BSN and AD perceptions were minimal

- Increase Student Learning

- BSN thought $13(68 \%)$ and AD thought 12 (63\%) strategies "effective"

- Diploma schools did not think any (0\%) were "effective"

- All 3 schools used the strategies "frequently" they perceived to be "effective"

- Strategies both "Frequently" Used and "Effective"

- 12 for BSN, 11 for AD, 0 for diploma

- BSN used CAI to "replace traditional" instruction

\section{Comparison of Effectiveness of Strategies for Learning}

The first analysis in this series compared the means of BSN, AD and diploma schools to see if there were differences in the ways the three types of schools perceived the "effectiveness" of the strategies for increasing student learning (See Appendix Table C 14). Once again, a mean of 3.00 or above was used to differentiate between strategies that were perceived to be "effective." The findings showed that there were minor differences in the way BSN and AD schools perceived the effectiveness of the strategies and that, while diploma schools rated all of the strategies as less "effective"than the other two, the patterns of the means for all three types of schools were similar. Stated differently, BSN schools perceived 13 , or $68 \%$ and AD schools thought 12 , or $63 \%$ of the 
strategies were "effective." None of the means for the diploma schools were above 3.00. The means for effectiveness for student learning were higher than the means for frequency of use and, as with the entire group, there was less of a variance between the means. In general, the strategies that were perceived to be most "effective" for increasing learning were the same strategies identified by all three types of schools as the ones they used most "frequently" (See Appendix Table C 14).

When the findings for "frequency" of use and perceived "effectiveness" were examined together, they showed that BSN schools had 12 and AD schools had 11 strategies that were both "frequently" used and perceived to be "effective" (See Table 15). The only difference between the two types of schools was that BSN schools used CAI to "replace traditional" instruction "frequently" and perceived it to be "effective" while AD schools did not. While diploma schools used 9 strategies "frequently," they did not perceive any to be "effective" for increasing student learning. 
Table 15. Integration Strategies That Were Frequently Used and Perceived to be Effective for Increasing Student Learning by BSN and AD Schools $(\mathrm{n}=77)$

\begin{tabular}{llllll}
\hline Strategy & & $\begin{array}{c}\text { BSN }(\mathrm{n}=25) \\
\text { Frequently }\end{array}$ & $\begin{array}{r}\text { BSN } \\
\text { Effective }\end{array}$ & $\begin{array}{l}\text { AD }(\mathrm{n}=48) \\
\text { Frequently }\end{array}$ & $\begin{array}{c}\text { AD } \\
\text { Effective }\end{array}$ \\
\hline $\mathrm{A}$ & Pretest & 1.60 & 2.58 & 1.71 & 2.48 \\
$\mathrm{~B}$ & Explain Objectives & $2.68^{* *}$ & $3.33^{* *}$ & $2.75^{* *}$ & $3.17^{* *}$ \\
$\mathrm{C}$ & Explain CAI & $3.00^{* *}$ & $3.40^{* *}$ & $2.94^{* *}$ & $3.33^{* *}$ \\
$\mathrm{D}$ & Motivate & $3.04^{* *}$ & $3.29^{* *}$ & $3.06^{* *}$ & $3.38^{* *}$ \\
$\mathrm{E}$ & Integrate & $3.52^{* *}$ & $3.25^{* *}$ & $3.29^{* *}$ & $3.31^{* *}$ \\
$\mathrm{~F}$ & Individual & $3.60^{* *}$ & $3.21^{* *}$ & $3.63^{* *}$ & $3.27^{* *}$ \\
$\mathrm{G}$ & Small Group & $2.52^{\wedge}$ & 2.75 & 2.44 & 2.90 \\
$\mathrm{H}$ & Whole-class & 2.52 & 2.79 & 2.08 & 2.69 \\
$\mathrm{I}$ & Technical Support & $3.68^{* *}$ & $3.60^{* *}$ & $3.79^{* *}$ & $3.63^{* *}$ \\
$\mathrm{~J}$ & Instructional Support & $3.68^{* *}$ & $3.58^{* *}$ & $3.90^{* *}$ & $3.71^{* *}$ \\
$\mathrm{~K}$ & Post-instruction & $2.80^{* *}$ & $3.21^{* *}$ & $2.73^{* *}$ & $3.17^{* *}$ \\
$\mathrm{~L}$ & Follow-up & $3.36^{* *}$ & $3.13^{* *}$ & $2.92^{* *}$ & $3.15^{* *}$ \\
$\mathrm{M}$ & Required & $2.96^{* *}$ & $3.25^{* *}$ & $3.02^{* *}$ & $3.27^{* *}$ \\
$\mathrm{~N}$ & Optional & 2.28 & 2.57 & 2.50 & 2.88 \\
$\mathrm{O}$ & Course Exam & $2.68^{* *}$ & $3.22^{* *}$ & $3.10^{* *}$ & $3.21^{* *}$ \\
$\mathrm{P}$ & Embedded & 1.80 & 2.74 & 1.48 & 2.31 \\
$\mathrm{Q}$ & Replace Traditional & $2.52++$ & $3.00++$ & 2.38 & 2.77 \\
$\mathrm{R}$ & Alternative & 1.72 & 2.96 & 2.44 & 2.96 \\
$\mathrm{~S}$ & Evaluate CAI & 2.36 & 3.04 & 2.15 & 3.04 \\
$\mathrm{Key}$ & & & & &
\end{tabular}

Key:

$\wedge$ Frequently used but not perceived effective

** Frequently use and perceived effective by BSN and AD schools

++ Frequently used and perceived effective by BSN schools only 
Comparison of Effectiveness of Strategies for Increasing Student Learning by Institution

- Public 12 (63\%) and private 9 (47\%) of 19 strategies were "effective"

- Public 5 and private 2 of the post-instruction strategies were effective

- Strategies that are "Frequently" Used and "Effective" (9)

- "Explain CAI, explain objectives, integrate, motivate, individual mode, technical and instructional support, required, and course exam"

- Public only " post-instruction and "follow-up"

A comparison of the means of the effectiveness of the strategies for increasing student learning based on the type of institution showed that public schools perceived 12 , or $63 \%$ of the integrations strategies were "effective" and public schools perceived 9 , or $47 \%$ of the 19 strategies were "effective" (See Appendix Table C 15). Both types of institutions perceived the four preinstruction strategies (explain objectives, explain CAI, integrate, and motivate) and the three mid-instruction strategies (technical and instructional support and individual) were "effective." However, for the post-instruction strategies, public schools perceived the five 5 strategies "required, post-instruction, follow-up, course exam, and evaluate CAI" were "effective"while private schools only thought "required and course exam" were "effective" strategies.

When the data was combined to identify the strategies that were both "frequently" used and "effective," the findings showed that public schools had 11 strategies and private schools had 9 strategies out of 19 that met both criteria. The nine that were shared by both included the "the four preinstruction strategies, individual mode, technical and instructional support, required, and course exam." The strategies that were both "frequently" used and perceived to be "effective" by public schools only were "post-instruction" and "follow-up." 
Comparison of Effectiveness of Strategies for Increasing Student Learning by School Size

- Medium and large 13 (68\%) and small 8 (42\%) were "effective"

- Small only thought 2 post-instruction strategies were "effective"

- Only medium "small group" mode

- Only large "alternative" method of restudying

- Strategies "Frequently" Used and "Effective"

- "Explain CAI, motivate, integrate, individual, technical and instructional support, and required" by all three

- Medium and large "explain objectives, post-instruction, and follow-up"

- Medium "small group"mode of instruction

- Large "evaluate CAI"

A third analysis was conducted to compare how the three sizes of schools evaluated the "effectiveness" of the strategies (See Appendix Table C 16). In general, the medium size and large schools rated the strategies higher than the small schools. More specifically, medium and large schools perceived 13 , or $68 \%$ and the small schools only thought 8 , or $42 \%$ were "effective" for increasing student learning.

As noted in Appendix Table C 16, the 4 preinstruction strategies were rated as "effective" by the medium and large schools but the small schools did not perceive the "pretest" or the "explain objectives" strategies were "effective." All three sizes of schools rated the three mid-instruction strategies of "technical and instructional support" and "individual" mode as "effective"but medium size schools also thought the "small group" mode was effective. Among the post-instruction strategies, small schools perceived "required" and "course-exam" were "effective" while medium and large schools both rated "required, post-instruction, follow-up, course exam and evaluate CAI" 
as "effective" strategies. Only the large schools rated providing "alternative" instruction as an "effective"strategy.

When the data was combined, the findings showed that 7 strategies were both "frequently" used and perceived to be "effective" by all three sizes of schools. The strategies included "explain CAI, motivate, integrate, individual, technical and instructional support, and required.” The three strategies that were both "frequently" used and "effective," according to medium and large schools included, "explain objectives, post-instruction, and follow-up." One strategy was "frequently" used and "effective" for medium size schools only was the use of the "small group" mode of instruction and the one that was "frequently" used and "effective" for large schools only which was to "evaluate CAI."

In summary, a comparison of the ways in which the three types of schools perceived the effectiveness of the strategies for increasing student learning showed that both BSN and AD schools perceived 11 strategies were "effective" for increasing student learning. Only the BSN schools rated the use of CAI to "replace traditional" instruction as "effective." The means for BSN and $\mathrm{AD}$ schools were close and the patterns of placement of the strategies were similar. Diploma schools did not perceive any of the strategies were "effective." In general, the strategies that were perceived to be most "effective"were also the ones that were also "frequently" used by all three types of schools.

The findings based on the type of institution showed that public schools rated 12 strategies as "effective" while private schools rated 9 strategies as "effective." Both institutions rated the same pre and mid-instruction strategies as "effective," but public schools thought 5 of the postinstruction strategies were "effective" and private schools only thought 2 strategies were. The 
differences were in the strategies of "post instruction, follow-up and course exam." There were 9 strategies that were rated as both "frequently" used and "effective" by both public and private schools and two additional ones by public schools alone.

The comparison of the three school sizes showed that medium and large schools rated the same number of strategies as "effective" while small schools were much less enthusiastic about the "effectiveness" of the strategies. The most outstanding feature of the findings was that the strategies the small schools did not think were "effective" and also did not use "frequently" were not related to a lack of resources but probably reflected their thoughts, values and practices.

Comparison of Effectiveness of the Strategies for Decreasing Costs

- No strategies were "effective" for decreasing costs (0\%)

- Type of School

- BSN and AD rated "replace traditional" high but diploma ranked it last

- BSN and AD rated "small group" as one of the lowest for decreasing costs

- Type of Institution.

- Public rated all 8 higher than private

- Private rated use of CAI to "replace traditional" high but almost never used it

- Public rated "technical" and "instructional" support highest while private rated them as the two lowest

- School size

- Minor differences in small, medium and large ratings of "effectiveness"

\section{Comparison of Effectiveness of Strategies for Costs}

The data was analyzed to compare how "effective"participants from the three different types of schools perceived the strategies were for decreasing costs (See Appendix Table C 17). The 
findings showed that none of the schools perceived the strategies were "effective" for decreasing costs. The differences between the means for the BSN and AD schools were minimal, but as before, the means for the diploma schools were much lower. Some unusual findings were noted were that while none of the strategies met the criteria of being "effective," BSN and AD schools perceived the strategy of using CAI to "replace traditional" instruction as one of the highest for reducing costs but the diploma schools ranked it near the bottom. Another was that BSN and AD schools perceived the "optional" strategy was the least "effective"of the eight while diploma schools ranked it as the top strategy. Both AD and BSN schools ranked the "individual mode" of instruction as one of the higher strategies for reducing costs but rated the "small group" and "whole class" modes as near the bottom.

The data was analyzed to compare participants" perceptions of "effectiveness" of the strategies for decreasing costs by the types of institution (See Appendix Table C 18). Again, none of the strategies had a mean above 3.00 but the public schools had higher means than private schools for all eight strategies. When the means were placed in rank order from the highest to the lowest, the strategies formed a somewhat similar pattern with only minor differences. Several of the findings were unusual. For instance, private schools ranked the "replace traditional" instruction strategy as next to the highest strategy for reducing costs but indicated by their answers to the second question that they seldom used it. There was also a difference in how public and private schools perceived the "effectiveness" of the "technical" and "instructional support"strategies. Public schools placed them near to top while private school participants ranked them near to bottom of the eight strategies. The findings were of particular interest because both private and public schools indicated that "technical" and "instructional support" were the two most "frequently" used 
strategies of the eight. This may be the first finding that indicated a difference in the resources of the schools influenced the way the participants perceived the "effectiveness" of the strategy.

When the three sizes of schools were compared to see how "effective" they perceived the strategies were for decreasing costs (See Appendix Table C 19) the findings showed that while none of the strategies were "effective," there was a step-up pattern in which small schools rated 7 of 8 strategies lower than medium size schools and medium size schools rated 7 of 8 strategies lower than large schools. Several points of interest were noted, such as all three schools rated the use of an "individual mode" of instruction as the top strategy on the list for reducing costs, which is understandable, but they also rated the strategies of providing "technical" and "instructional support" next. The later findings are much more difficult to understand than the first.

Comparison of Effectiveness of Strategies to Decrease the Students' Time

- Type of School

- BSN and AD rated 4 (57\%) of 7 as "effective" but diploma none (0\%)

- "Technical" and "instructional" 2 most "effective" by all three

- "Small group" and "whole class" least "effective" for decreasing students" time

- Type of Institution

- Public 4 (57\%) and private 3 (43\%)

- Schools Size

- Large 5 (71\%), medium (57\%) and small 3 (43\%)

\section{Comparison of Effectiveness of Strategies for Students' Time}

The data was analyzed to determine how the three different types of schools perceived the "effectiveness" of the strategies for decreasing the students' time (See Appendix Table C 20). The 
findings showed that the means for BSN and AD schools were generally higher than the means for the diploma schools and that both BSN and AD schools rated 4 strategies as "effective" while diploma schools did not think any were. There were several findings of interest such as all three types of schools identified the strategies of provide "technical" and "instructional" support as the two highest ranked strategies for saving the students' time and all three schools thought the use of "small group" and "whole class" were the two least "effective"strategies for that purpose.

An analysis of the same data was conducted that compared public and private schools (See Appendix Table C 21) and the findings showed that the means for public schools were slightly higher than private schools but that public schools rated 4 strategies as "effective" and private schools thought 3 strategies were. There were a few variations in the placement of the strategies but, in general, the patterns were similar. Both thought "explain CAI, provide technical and instructional support" were "effective" strategies for decreasing the students' time.

Another analysis compared the three sizes of schools (See Appendix Table C 22) and the findings showed that the means for large schools were slightly higher than the medium size schools and the means for medium schools were slightly higher than the small schools. Large schools thought 5 strategies were "effective," medium size schools thought 4 were and small schools thought 3 strategies were "effective." Once again, provide "technical" and "instructional support" were perceived to be the most "effective" strategies for saving the students' time and the use of the "small group" and "whole class" modes of instruction were the least "effective"strategies. 
Comparison of Effectiveness of Strategies for Decreasing the Teachers' Time

- Type of School

- BSN thought $4(40 \%)$ and $\mathrm{AD} 3(30 \%)$ and diploma none $(0 \%)$

- "Replace traditional" was not "effective" for saving teachers' time by any

- "Individualized" instruction was rated high by all three

- Type of Institution

- Public rated $3(30 \%)$ and private rated $2(20 \%)$ "effective"

- Both thought "technical" and "instructional" was the most "effective"

- Public thought "individual" mode of instruction

- School Sizes

- Medium rated $4(40 \%)$ large $2(20 \%)$ and small none $(0 \%)$ "effective"

- Medium thought "replace traditional" and "individual" were effective

Comparison of Effectiveness of Strategies to Decrease Teachers' Time

The data was analyzed to determine if the three types of schools perceived the "effectiveness" of the strategies differently (See Appendix Table C 23). The findings showed that the BSN schools rated 4 , or $40 \%$ of the strategies, $\mathrm{AD}$ schools rated 3, or $30 \%$ as "effective," but the diploma schools did not think any of the strategies were "effective"for reducing the teachers' time. All of the means for the diploma schools tended to be lower than the means for the BSN and AD schools. While the BSN schools thought including CAI content on an in-class "course exam" was the third highest strategy, diploma schools rated it as one of the least "effective"ways to save the teachers' time. Also, the BSN and AD schools perceived assigning CAI as an "optional" assignment was one of the least "effective"strategies of the ten while diploma schools rated it as 
one of the highest. None of the schools thought the use of CAI to "replace traditional" instruction strategy was an "effective"way to decrease the teachers' time.

When an analysis of the same data was conducted based on the type of institution (See Appendix Table C 24), the findings showed that public schools had 3 strategies with a mean above 3.00 and private schools had 2 above that number. In general, there were few differences in the means of the two groups and the pattern of the strategies that were perceived of as "effective" were similar. The schools agreed that providing "technical" and "instructional support" and an "individual" mode of instruction were the three most "effective"strategies for decreasing the teachers' time. While public schools rated using CAI to "replace traditional" instruction higher than private schools, but the strategy was not perceived to be "effective" by either group.

The third analysis in the series looked at the means for the three different sizes of schools (See Appendix Table C 25). The findings showed that small schools did not think any of the strategies were "effective," but medium size schools thought 4 , or $40 \%$ were and large schools thought 2 , or $20 \%$ were. All three groups of schools ranked provide "technical" and "instructional support" and "individual" mode of instruction as the top three most "effective" strategies for saving the teachers' time. 
Comparison of Correlations of Strategies for Increasing Student Learning

- Types of School

- 7 significant BSN and AD also "frequently"used and "effective"

- 5 in common "explain CAI, explain objectives, motivate, integrate, course exam"

- BSN only "required \& individual mode"

- AD only "technical support" and "follow-up"

- Type of Institution

- Public (6) "explain CAI, explain objectives, integrate, motivate, course exam, follow-up"

- Private (5) "explain CAI, explain objectives, integrate, motivate, individual"

- School Size

- Small (6) “explain objectives, integrate, motivate, individual, instructional support and required"

- Medium(3) "individual, small-groupand course exam."

- Large (8) "explain CAI, explain CAI, integrate, motivate, course exam, follow-up, post-instruction, evaluate CAI"

\section{Comparison of Correlations}

Since a high correlation figure did not necessarily mean the strategy was "frequently" used or perceived to be "effective," only those strategies with a statistically significant correlation that also met the previously established criteria of a mean of 2.50 or above for "frequency" of use and a mean of 3.00 or greater for "effectiveness" were included in the discussion. Also, the diploma schools were not included in the analysis because so many of the findings for the group were negligible. 


\section{Comparison of Correlations for Learning}

The findings of the Pearson $r$ correlations showed that 14 of the strategies were statistically significant for the BSN schools as compared to 15 for $\mathrm{AD}$ schools at a $\mathrm{p}<0.01$ level of significance (See Appendix Table C 26). Only 7 of the significant correlations also met the criteria of being "frequently" used and "effective" for both the BSN and the AD schools. For the BSN schools, the strategies that had strong correlations between 0.62 to 0.50 and met the other three criteria included "explain CAI, integrate and course exam." The 4 strategies with weaker correlations between 0.36 to 0.31 that also met the other two criteria were "explain objectives, motivate, individual mode and required." Associate schools also had 7 statistically significant correlations that also met the other two criteria. The 3 strongest correlations were between 0.51 to 0.46 and included "integrate, motivate, and course exam." The other 4 strategies that were significant correlations between 0.41 and 0.38 were "explain CAI, explain objectives, technical support, and follow-up." The strategies that met all three criteria for BSN schools only were "required" and the use of an "individual" mode of instruction. The 2 strategies that were exclusively significant for the AD schools were "followup and technical" support.

A similar analysis was conducted to examine the differences in the two types of institutions and the findings showed that both public and private schools had 13 statistically significant correlations at a $\mathrm{p}<0.01$ level of significance (See Appendix Table C 27). Of those, public schools had 6 and private schools had 5 strategies that also met the other criteria. In general, the correlations for private schools were stronger than the correlations for the public schools and particularly among the strategies that were both "frequently" used and "effective." The significant correlations for the public schools included "“explain CAI, explain objectives, integrate, motivate, 
course exam, and follow-up." Among the private schools, the strategies were "explain CAI, explain objectives, integrate, motivate, and an individual mode" of instruction. Only public schools had significant correlations for the strategies of "course exam and follow-up" while only private schools had a significant correlation for the strategy of "individualized" instruction.

A similar analysis was conducted to determine if there were differences in the correlations between three sizes of schools (See Appendix Table C 28) and the findings showed that there were 18 significant correlations for the small schools, 7 for the medium size schools and 16 for the large schools. There were considerable differences between the three sizes of schools and how many of the significant correlations were also "frequently" used and evaluated as "effective." For instance, small schools had 18 significant relationships but only 6 of those were "frequently" used and "effective," medium size schools had 7 significant correlations but only 3 that were also "frequently" used and "effective" and large schools had 18 significant correlations but only 8 that were also "frequently" used and "effective."

The statistically significant correlations for the strategies for the small schools were "explain objectives, integrate, motivate, individual, instructional support and required." The 3 strategies for medium size schools that also met the other criteria included "small group, individual mode and course exam." For the large schools, the strategies were "explain CAI, explain objectives, motivate, course exam, follow-up, post-instruction, and evaluate CAI.” 


\section{Comparison of Correlations for Decreasing Costs}

- 7 statistically significant but none were "effective"

- Type of School

- 4 for BSN, 0 for AD but none met all three criteria

- Type of Institution

- 1 for public, 2 private but none met all three criteria

- Schools Size

- 3 small, 1 medium, 3 large but none met all three criteria

\section{Comparison of Correlations for Costs}

The Pearson $r$ correlation was calculated to compare the strength of the relationships between the frequency of use and the perceived effectiveness of the strategies based on the type of schools (See Appendix Table C 29). Once again, the strategies that were statistically significant needed to meet all three criteria of "frequency" of use and "effectiveness" to be included in the discussion. Since none of the strategies were perceived to be "effective" for decreasing costs, none met all three criteria. The findings did show that BSN schools had 4 strategies that were significant at a $\mathrm{p}<0.01$ level of significance and $\mathrm{AD}$ schools did not have any. The strategies that were significant for BSN schools were "individual, required, small-groupand whole class." A similar situation existed when the data was analyzed based on the type of institution (See Appendix Table C 30). The findings showed that public schools only had 1 statistically significant strategy which was a "whole class" mode but it was not "frequently" used or perceived to be "effective." Private schools had 3 statistically significant strategies which included "individual, required and replace" traditional instruction. The findings for the analysis based on the three sizes of schools showed that 
small schools had 3, medium size schools had 1 and large schools had 3 strategies that were statistically significant (See Appendix Table C 31). The significant strategies for the small schools were "whole class, required, and replace" traditional instruction. Of those, none were "frequently" used or "effective. For the medium size schools, only the "required" strategy was significant and for the large schools, "whole class, optional, and replace" traditional instruction was significant but none were "frequently" used or "effective."

Comparison of Correlations for Decreasing the Students' Time

- Type of School

- 1 for BSN, "explain CAI," and 1 for AD, "explain objectives"

- Type of Institution

- 2 for public but none met all three criteria

- 1 for private, "explain CAI"

- School Size

- 1 for small, "explain CAI," 1 for medium, "instructional support," 1 for large, "small group"

\section{Comparison of Correlations for Students' Time}

The Pearson $\mathrm{r}$ correlation was calculated to determine the relationship between the "frequency" of use and the perceived "effectiveness" of the strategies based on the type of schools (See Appendix Table C 32). The findings showed that BSN schools had 1 statistically significant correlation ( $\mathrm{p}<0.01$ level of significance), which was "explain CAI" that also met the other criteria. Associate schools had 3 statistically significant correlations but only the strategy, "explain objectives" met the other criteria. Public schools had 2 statistically significant findings but neither 
one met the other criteria (Appendix Table C 33). Private schools had 3 statistically significant strategies and one, "explain CAI" met the other criteria. Small schools had 4 strategies that were significant but only "explain CAI" met the other criteria (See Appendix Table C 34). Medium schools rated the strategy of "instructional support" with a significant $(-0.46)$ correlation and it was "frequently" used and "effective." Large schools thought the strategy of "small group" was significant and "frequently" used but not "effective."

Comparison of Correlations for Decreasing the Teachers' Time

- Type of School

- 3 for BSN but only "individual" met all three criteria

- 2 for AD but none met all three criteria

- Type of Institution

- 1 for public and 3 for private but none met all three criteria

- School Size

- 4 for small, 2 for medium, 4 for large but none met other criteria

\section{Comparison of Correlations for Teachers' Time}

The results of the Pearson $r$ correlations showed that there were three statistically significant relationships for BSN schools, but only "individual mode" of instruction with a (-0.31) correlation met the other criteria (See Appendix Table C 35). There were 2 significant correlations for AD schools but none that met the other criteria. There was 1 significant relationship for public schools and 3 for private but none met the other criteria (See Appendix Table C 36). A similar situation existed for the three sizes of schools. Small schools had 4 significant correlations, medium had 2 and large had 4 but none that were "frequently" used and "effective" (See Appendix Table C 37). 
Key Features of Schools and Educators

Key Features of Schools

A further exploration of the "key features" of the schools that effectively integrate CAI, the next part of the study examined the computer resources of the various schools. A discussion of the findings will follow.

Computer Lab in the SON

- $69 \%$ had and $31 \%$ did not have a computer lab in the SON

- $68 \%$ of all BSN, $68 \%$ AD schools and $100 \%$ diploma schools

- $71 \%$ of all public and $64 \%$ of all private

- $68 \%$ of all small, $60 \%$ of the medium and $83 \%$ of the large

Schools with a Computer Lab in the SON

The fourteenth question of the ICAI Questionnaire asked participants about the presence of a computer lab that was located in the school of nursing. The findings are available in the appendix (See Appendix Table C 38) and showed that out of the 109 sample schools, $69 \%$ had a computer lab in the SON and 31\% did not. There was one non-response.

The data was analyzed further to determine the differences among the various types of schools and showed that $40 \mathrm{AD}, 30 \mathrm{BSN}$ and all five diploma schools had a computer lab in the school of nursing. A calculation of the percentages showed that $68 \%$ of the BSN and $68 \%$ of the AD and $100 \%$ of the diploma schools had a lab in the SON. The analysis by the types of institutions showed that $71 \%$ of the public and $64 \%$ of the private schools had a computer lab in the school of nursing (See Appendix Table C 39). When the data was examined for differences based 
on school sizes, the findings showed that $68 \%$ of the small, $60 \%$ of the medium sized schools and $83 \%$ of the large schools had a lab (See Appendix Table C 40).

In summary, over twice as many sample schools reported they had a computer lab in the SON as did not. The findings showed that equal numbers of AD and BSN schools had a lab and all of the diploma schools in the sample had a lab in the school of nursing. A slightly larger percent of the public schools had a lab than private schools. The findings also showed that a much higher percent of large schools had a lab than small or medium schools and that a larger percent of small schools had a lab than medium size schools. 


\section{Locations of Computers}

- $29 \%$ in the SON, $29 \%$ library, $21 \%$ another department and $21 \%$ students purchase their own computers

- CAI-users vs. Non-users

- CAI-user schools $72 \%$ vs. non-users $28 \%$ of the responses

- CAI-users had more computers in the SON than non-users

- Non-users had more in library and had students purchase their own

- Type of School

- BSN highest library and lowest in another department

- BSN have students purchase more than AD or diploma

- AD highest in SON and library

- Diploma $40 \%$ in SON and $40 \%$ library

- Type of Institution

- Public $77 \%$ and private $23 \%$ of responses

- Public highest in SON followed by the library

- Private highest in library followed by having students purchase their own

- School Size

- Small and medium highest in the library, large in the SON

\section{Locations of Computers}

Question 15 of the ICAI Questionnaire asked participants to indicate with an "X" where the computer's students use are located in the school. The four options that were provided were the "school of nursing," "another department," "library," or "students purchase their own." Of the 303 total responses to the question, $29 \%$ indicated their school had computers located in the SON, $29 \%$ had computers in the library, $21 \%$ in another department and $21 \%$ said they had students purchased their own computers (See Appendix Table C 41). The findings also showed that CAI-user schools' 
responses made up $72 \%$ of the total and non-user schools' responses amounted to $28 \%$ (See Appendix Table C 42). A breakdown of where the CAI-users schools' computers were located showed that a majority of the computers were located in the school of nursing (30\%) followed by the library (29\%). In contrast, non-users reported most of their computers were located in the library (31\%) followed by the school of nursing (26\%) and they reported having "students purchase their own computers" more often than CAI-user schools.

When the data was analyzed to compare the different types of schools, the findings showed that most, or $52 \%$ of the 303 responses, were from AD schools, $45 \%$ were from $\mathrm{BSN}$ and $3 \%$ were from diploma schools (See Appendix Table C 43). Associate schools had equal numbers of computers (29\%) in the SON and the library. For the BSN schools, 29\% reported having computers in the library and $28 \%$ had them in the SON. The five diploma schools indicted $40 \%$ of their computers were in the SON and $40 \%$ were in the library. It was also noted that a larger percent of the BSN schools (25\%) reported they had students purchase their own computers than AD (18\%) or diploma schools (10\%).

The analysis of differences based on the type of institution showed that $77 \%$ of the participants from public schools reported computers in the four locations as compared to only $23 \%$ from private schools (See Appendix Table C 44). Public had 30\% of their computers in the SON and $29 \%$ were in the library. Most of the computers for the private schools, or $31 \%$ were located in the library and $27 \%$ had students purchase their own.

The findings for the different sizes of schools showed that $39 \%$ of the medium, $33 \%$ of the small schools and $28 \%$ of the large schools reported having computers in the four locations (See Appendix Table C 45). The majority, or 30\% of the small and medium size schools reported 
having computers in the library and $32 \%$ of the large schools reported having computers in the SON.

In summary, of the 303 total responses, $29 \%$ of the respondents indicated their schools had computers in the SON and the library and $21 \%$ had computers in another department or had students purchase their own. In all, $72 \%$ of the responses were from CAI-users and $28 \%$ were from non-users. Schools that used CAI had slightly more computers in the SON and non-users had more computers in the library and had students purchase their own computers.

The first comparison showed that more AD school participants responded to the question than BSN or diploma schools. All three types of schools reported most of the computer's students used were located in the SON or the library. The BSN schools had students purchase their own computers more often that $\mathrm{AD}$ or diploma schools and only reported $18 \%$ of their computers were located in another department. Diploma schools had a heavier concentration of their computers in the school of nursing and library. Public schools made up $77 \%$ of the responses and only $23 \%$ were from private schools. Public schools reported most of their computers were in the SON followed by the library and private schools had most of their computers in the library followed by having students purchase their own. The comparison of the three sizes of schools showed that medium size schools responded to the question more than small or large schools. Small and medium size schools reported having computers in the library more than large schools where most of the computers were located in the school of nursing. 


\section{Computer Resources}

- Sample schools owned 12,265 computers $(M=113)$

- 50\% were in "another department," 36\% in "library" and 14\% "SON"

- CAI-users owned 3 times as many in "SON," twice as many in "library" and "another department" as non-users

- Type of School

- BSN owned 55\%, AD owned 44\% and diploma owned 1\%

- BSN owned 6,441 (M = 52), AD owned 5,441(M = 31) and diploma owned 114 $(\mathrm{M}=9)$ computers

- BSN 50\% in "library" and "another department" and 11\% in "SON"

- AD most in "another department" and 17\% in SON

- Diploma equally "SON" and "library"

- Types of Institutions

- Public owned $87 \%$ and private owned $13 \%$

- Most in "another department" and a few in "SON"

- School Sizes

- Medium owned 46\%, large owned 29\% and small owned 26\%

- Small most in "another department"

- Medium equal in "another department" and "library"

- Large most in "another department" and SON

\section{Computer Resources}

Question 16 of the ICAI Questionnaire asked participants to "type in the number of computers that were available for students to use that were located in each of three departments of the school." The options included the school of nursing, another department or the library and the findings showed that the 109 participants reported owning $12,265(\mathrm{M}=113)$ computers. The 
locations of the computers were $50 \%$ in another department, $36 \%$ in the library and the other $14 \%$ were in the school of nursing.

The data was analyzed to determine if CAI-user schools differed from non-user schools in terms of the number of computers they owned or where they were located (See Appendix Table C 46). The findings showed that of the 1,736 computers that were located in the SON, CAI-user schools owned $76 \%$ and of the 6,156 computers that were located in another department, CAI-user schools owned $65 \%$, and of the 4,394 computers located in the library, CAI- user schools owned $70 \%$ of them (See Appendix Table C 47).

When the same data was evaluated based on the three types of school, the findings showed that BSN schools owned 6,710 computers, AD schools owned 5,441 and diploma schools owned 114 computers. The means were more meaningful and showed that the BSN schools owned a mean of 52 computers while AD schools had a mean of 31 and the mean for the diploma schools dropped to 9 computers. Bachelor schools owned 55\%, AD schools owned $44 \%$ and diploma schools owned $1 \%$ of the total computers (See Appendix Table C 48). The locations of the computers also varied considerably by the type of school. For instance, BSN schools had almost equal percentages of computers located in "another department" (45\%) and the "library" (44\%) with only $11 \%$ in the school of nursing. The AD schools reported a large majority, or 57\% of the computers were located in "another department," while $26 \%$ were in the library and only $17 \%$ were in the school of nursing. Diploma schools reported almost equal numbers of computers in the three different departments. More specifically, 36\% were in the SON, 35\% in "another department" and another 29\% were in the library. 
Analysis of the data to compare the two types of institutions showed that public schools owned $87 \%$ and private schools only owned $13 \%$ of the total computers. The locations of the computers were almost identical (See Appendix Table C 49). Public schools reported 50\% and private reported $49 \%$ of the computer were located in "another department" followed by $36 \%$ (public) and 33\% (private) in the library and a much smaller percent in the school of nursing. When the data was analyzed by the different sizes of schools, the findings showed that medium size schools owed more computers than large or small schools (See Appendix Table 50). Small schools owned $26 \%$, medium owned $46 \%$ and large schools owned $29 \%$ of the total computers. Small schools reported $60 \%$, medium schools reported $46 \%$ and large schools reported $48 \%$ of their computers were located in "another department." All three sizes of schools reported the next highest number were located in the "library," which was followed by the SON. Medium size schools reported almost half, or $42 \%$ of their computers were located in the "library." Large schools had more computers in the SON than either small or medium size schools. 
Full and Part-time Technical and Instructional Support Personnel

- 278 full and 191 part-time technical and 158 full and 85 part-time instructional

- CAI-user and non-user schools

- CAI-users 5 times as many full and part-time technical and 9 times as many full and part-time instructional personnel as non-user schools

- CAI-user higher full-to-part-time ratio of both technical and instructional than nonuser schools

- Type of School

- AD more full and part-time technical than BSN or diploma

- AD more full and part-time instructional than BSN schools

- BSN higher ratio of full-to-part-time technical and instructional than AD

- Type of institution

- Public 5 times as many technical and 3 three times instructional

- Same ratio of full-to-part-time technical support personnel

- Public higher ratio of full-to-part-time instructional

- School Sizes

- Large more full-time technical and small more part-time technical

- Medium more full-time and large more part-time instructional

- Medium highest ratio of full-to-part-time technical

- Small highest ratio of full-to-part-time instructional

\section{Technical and Instructional Support Personnel}

Question 17 asked participants to identify the number of technical and instructional support personnel employed by the school. In response, participants were asked to type a number from 0 to 999 next to each of the four options which included: full-time technical (FTT) and part-time technical (PTT) support personnel and full- time instructional (FTI) and part-time instructional 
(PTI) support personnel. The analysis showed that the schools employed a total of 278 FTT, 191 PTT and 158 FTI and 85 PTI personnel (See Appendix Table C 51).

The data was analyzed for a difference in the number of support personnel employed by CAI-user and non-user schools. The findings showed that CAI-user schools employed $86 \%$ and non-users employed $14 \%$ of the total full-time technical support personnel and that CAI-user schools employed $84 \%$ and non-user schools employed $16 \%$ of the total part-time technical support personnel. Also, CAI-user schools employed $91 \%$ full-time and $88 \%$ part-time instructional support personnel compared to $9 \%$ full-time and $12 \%$ of the part-time instructional support personnel. Another compared the ratio of full-to-part-time support personnel employed by the two groups. The findings showed that CAI-user schools employed $60 \%$ full-time and $40 \%$ part-time technical and non-user schools employed 55\% full-time and 45\% part-time technical employees (See Appendix Table C 52). Similarly, the CAI-user schools employed 66\% full-time and 34\% part-time instructional support employees and non-user schools employed $60 \%$ full and $40 \%$ parttime employees. The ratio of full-to-part-time employees was higher for the CAI-user schools. A second analysis compared the three types of schools and the findings showed that AD schools employed $62 \%$, BSN schools employed $37 \%$ and diploma schools employed $1 \%$ of the fulltime and part-time technical support personnel (See Appendix Table C 53). Associate schools also employed $56 \%$ full and part-time instructional personnel as compared with $41 \%$ for the BSN schools. However, the findings showed that BSN schools had a higher ratio of full-to-part-time employees than the $\mathrm{AD}$ or diploma schools for both technical and instructional support personnel (See Appendix Table C 54). The finding was supported by the fact that BSN schools employed $60 \%$ full to $40 \%$ part-time while AD schools employed $50 \%$ full to $50 \%$ part-time technical support 
personnel. For the instructional support personnel, BSN schools employed $67 \%$ full and $33 \%$ parttime while AD schools only employed $62 \%$ full and 38\% instructional support personnel. Diploma schools had a 1 to 1 ratio of full-to-part-time employees for technical support and did not employ any instructional support personnel.

A third analysis compared the two types of institutions and the findings showed that public schools employed $84 \%$ and private schools only employed $16 \%$ of the total full and part-time technical support personnel (See Appendix C 55). Similarly, public schools employed 75\% and private schools employed $23 \%$ of the full and part-time instructional support personnel reported. The comparison of the ratio of full-to-part-time support personnel employed by the two types of institutions showed that public and private schools had the same ratio for technical support personnel but public schools held a moderate lead over private schools for the ratio of full-to-parttime instructional support personnel employed.

Another analysis compared the three sizes of schools and the findings showed that large schools employed $37 \%$, medium schools employed $33 \%$ and small schools employed $29 \%$ of the full-time technical support personnel (See Appendix Table C 56). Small schools employed the highest percent of part-time technical support personnel. Medium schools employed the highest percent of full-time instructional support personnel with 39\% and large schools employed the highest percent of part-time instructional support personnel with $42 \%$. When the ratios of full-topart-time employees were calculated, the findings showed that medium schools employed a higher ratio of full-to-part-time technical and small schools employed a higher ratio of full-to-part-time instructional support employees. 
In summary, the schools reported they owned over 12,000 computers and most were located in "another department" followed by the "library," and a small number were located in the "school of nursing." CAI-user schools had more computers in all three locations than non-user schools. Bachelor schools owned the most computers followed by AD schools and the diploma schools only owned $1 \%$ of the total computers. The BSN schools owned an average 52, AD schools owned an average of 31 and diploma schools 9. The locations of the computers were different for the three types of schools. While BSN schools had almost the same number of computers in "another department" and the "library" and very few in the SON, the AD schools reported a large majority in "another department," a lower number in the "library" and an even smaller percent in the "school of nursing." Diploma schools reported almost equal numbers of computers in the three different departments.

Another analysis showed that public schools owned over three times as many computers as private schools. Both types of institutions reported one half of their computers were in "another department" and a third in were in the "library" and a small number were in the "school of nursing." When the three different sizes of schools were analyzed, the findings showed that medium schools owned almost one half of the total number of computers and small and large schools owned almost $30 \%$ each. A majority of the computers in all three size schools were located in "another department." Medium size schools had almost one half of their computers in the "library." Large schools had the most computers located in the "school of nursing."

The findings for the full and part-time technical and instructional support personnel showed that the schools employed more technical than instructional support personnel and more full-time than part-time employees in both categories. CAI-user schools employed six times as many full- 
time and five times as many part-time technical support personnel and 10 times as many full-time and 7 times as many part-time instructional support personnel as non-user schools. The ratio of full-to-part-time support personnel that were employed by CAI-user schools was higher than for the non-user schools.

AD schools employed more of all four types of employees than BSN or diploma schools. For instance, AD schools had almost twice as many full and part-time technical employees as BSN schools and 6 times as many as the diploma schools. The difference for the number of instructional support employees was much smaller. Bachelor schools had a higher ratio of full-to-part-time employees in both categories than AD schools.

Public schools employed over 5 times as many full and part-time technical support personnel and 3 times as many full and part-time instructional support personnel as private schools. The public and private schools employed the same ratio of full-to-part-time technical support personnel but the pubic schools held a lead over the public schools for the ratio of full-to-part-time for instructional support personnel.

The analysis of the three school sizes showed that large schools employed more full-time technical support employees than medium and medium schools employed more than small schools. Small schools employed more part-time technical support personnel than the other two. Medium size schools employed more full-time instructional support personnel and large schools employed more part-time instructional support personnel than the others. When the ratios of full-to-part-time employees were analyzed, the findings showed that medium schools had the highest ratio for technical and small schools held a slight lead for instructional support personnel employed. 
- Percent of Teachers That Use CAI: Responses to Question 18

- Part “A” $648(\mathrm{M}=7.62)$ teachers used CAI

- More AD than diploma and more diploma than BSN

- More from private than public

- More from large than medium or small

- Part "B" - N = 109

- 28 said " $0 \% "$

- 50 said $1 \%$ to $50 \%$

- 78 said less than $50 \%$

- $25 \%$ said $80 \%$ to $100 \%$

- Type of School

- 17 BSN and 10 AD said "0\%"

- $3 \mathrm{BSN}$ and $27 \mathrm{AD}$ said $51 \%$ or more

- Type of Institution

- More public than private said " $0 \%$ "

- 26 public and 4 private said $51 \%$ or above

- School Sizes

- 17 small, 6 medium and 6 large said 51\% or above

\section{Key Features of Educators}

Question 18 A of the ICAI Questionnaire asked participants to type a number (0 to 999$)$ to indicate how many teachers from their schools used CAI and Part "B" asked them to identify what percent ( 0 to $100 \%$ ) of the faculty that number was. It was noted that eight more participants responded to part "A" and five more responded to part "B" of Question 18 as responded to Question 1 of the ICAI Questionnaire. The reason for the discrepancy was probably due to a 
difference in the wording of the questions. The first question asked participants if "they" used CAI in the past year and Question 18 asked participants to identify the number of "other" teachers at their school who used CAI to teach clinical decision-making.

The findings of the "A" part of Question 18 showed that 85 participants indicated a total of 648 teachers at their schools used CAI $(M=7.62)$. A breakdown by the type of school showed that diploma schools actually had a higher mean and median than the AD or BSN schools and that AD schools had a higher mean than the BSN schools (See Table 16). Another set of surprising findings showed that private schools had a much higher mean than public schools for the number of teachers who used CAI. The findings from a comparison of the three sizes of schools showed that large schools had a much higher mean for the number of teachers who used CAI than either medium or small schools. The means for the small and medium size schools were surprisingly close. 
Table 16. Measures of Central Tendency of Number of Teachers That Use CAI in Response to Question 18 A by Type of School, Type of Institution, Size of School (N=109)

\begin{tabular}{lccc}
\hline School & Mean & SD & Median \\
\hline Bachelor $(\mathrm{n}=45)$ & 5.51 & 8.88 & 3.00 \\
Associate $(\mathrm{n}=59)$ & 6.12 & 4.90 & 5.00 \\
Diploma $(\mathrm{n}=5)$ & 7.80 & 7.17 & 6.00 \\
& & & \\
Public $(\mathrm{n}=59)$ & 5.10 & 5.19 & 4.50 \\
Private $(\mathrm{n}=25)$ & 8.80 & 10.45 & 6.00 \\
& & & \\
Small $(\mathrm{n}=37)$ & 4.22 & 4.02 & 4.00 \\
Medium $(\mathrm{n}=43)$ & 4.60 & 5.04 & 3.00 \\
Large $(\mathrm{n}=29)$ & 10.14 & 9.94 & 3.00
\end{tabular}

In response to Part B of the question, 81 participants indicated that somewhere between " 1 and $100 \%$ " of the teachers at their schools used CAI while 28 participants typed either a " 0 " or left the space blank. Of the 81 positive responders, $60 \%$ were from $\mathrm{AD}$ schools, $35 \%$ were from $\mathrm{BSN}$ and 5\% were from diploma schools. When broken down by type of institution, $79 \%$ were from public and $21 \%$ were from private schools.

The data was analyzed and the findings were placed in a frequency distribution chart and polygon graph for the purpose of illustration. Both are available for review in the appendix (See Appendix Table C 57). A brief summary of the findings showed that 28 of the responders indicated that " $0 \%$ " of the teachers at their school used CAI and 50 responders indicated that between $1 \%$ and $50 \%$ used CAI. When combined, the figures showed that 78 participants out of 81 indicated 
less that $50 \%$ of the employees at their schools used CAI while at the other side of the spectrum, $25 \%$ indicated that between $80 \%$ and $100 \%$ of the teachers at their schools used CAI.

A frequency distribution broken down by the type of school showed that there was a large difference between the BSN and AD schools in terms of the percent of teachers who used CAI (See Appendix Table C 58). The BSN schools had 17 respondents who indicated "none" of the teachers at their schools used CAI while AD schools only had 10 such responses. Even more telling were the numbers at the other end of the spectrum that showed that AD schools had 27 responses that indicated $51 \%$ or more of the teachers at their schools used CAI while the BSN schools only had three responses at or above $51 \%$.

When the same data was analyzed to determine if there were differences based on the type of institution, the findings showed that 22 participants from public schools indicated " $0 \%$ " of the teachers at their schools used CAI as compared to only 6 from private schools. However, the public schools also had 21 participants who indicated that $100 \%$ of the teachers at their schools used CAI as compared with only 1 from a public school (See Appendix Table C 59). It was also noted that public schools had a total of 26 responses that indicated that $51 \%$ or above of the teachers used CAI as compared to only 4 such responses for the private schools. The third analysis to look at the three sizes of schools and the findings showed that small schools clearly had a larger number of responses that indicated a large percent of their teachers used CAI (See Appendix Table C 60). In particular, small schools had 17 responses of $51 \%$ or above, and medium and large schools each had 6 responses above $51 \%$.

In summary, the findings showed that a total of 648 teachers at the 109 participants' schools used CAI. Most of the responses were from AD and public schools. 
In response to Part A of the question, the findings showed that diploma school participants reported a larger number of teachers who used CAI than AD or BSN schools and that AD schools reported a larger number of CAI-users than BSN schools. The findings of a comparison of the types of institutions showed that private schools had many more CAI-user teachers than public schools. The third analysis showed that large schools reported many more teachers who used CAI than medium or small schools in the sample.

In response to question $18 \mathrm{~B}$, a frequency table and polygon graphic illustration showed that $72 \%$ of the respondents indicated that $50 \%$ or fewer of the teachers at their schools used CAI. At the other end of the spectrum, $25 \%$ of the schools indicated that between 80 and $100 \%$ of the teachers used CAI. When the findings were broken down by the type of school, they showed that AD schools had larger percentages of teachers who used CAI. The findings by the type of institution showed that public schools had many more responses that indicated $51 \%$ or more of the teachers used CAI. When the three sizes of schools were compared, small schools had almost three types as many responses over 51\% than either large or medium size schools. 
Key Features of Participants Who Use CAI

Roles of CAI-users

- Faculty $(n=97) \quad 68(72 \%)$ CAI-users

- 56\% BSN, 81\% AD and 60\% diploma

- $74 \%$ public and $57 \%$ private

- $71 \%$ small, $63 \%$ medium and $83 \%$ large

- Administrators $(\mathrm{n}=9) \quad 6(67 \%)$ CAI-users

- $5 \mathrm{AD}$ and $1 \mathrm{BSN}$

- 5 public and 1 private

- 2 small, 1 medium and 3 large

- Computer lab personnel $(n=3) \quad 1(33 \%)$ CAI-user

- BSN, private, medium school

- Genders

- Males (n=6) 4 (66\%) CAI-users

- $4 \mathrm{AD}$ and public

- 1 medium and 3 large

- Females $(n=102) 71(70 \%)$ CAI-users

- $80 \%$ AD, 57\% BSN and 60\% diploma

- $72 \%$ public and $63 \%$ private

- $80 \%$ large, $72 \%$ small and $61 \%$ medium

\section{Key Features of Participants}

The next part of the discussion focused on the "key features" of effective CAI users and explored the relationship between the personal characteristics of the participants and their use of

CAI. As noted previously, the sample $(\mathrm{N}=109)$ consisted of participants from $45 \mathrm{BSN}, 59 \mathrm{AD}$ and 5 diploma schools. In response to the demographic questions, or Question19 through 21 of the 
ICAI Questionnaire, participants were asked to indicate their role at the school, gender, age range, and educational background. The findings showed that 97 participants indicated they were faculty members, and of those, $72 \%$ used CAI and $28 \%$ did not. Of the 9 administrators, $67 \%$ used CAI and 33\% did not. There were 3 participants who identified their role as a "computer lab employee" and of those, 1 used CAI.

When broken down by the type of school, the findings showed that $56 \%$ of the faculty from BSN, $81 \%$ of the $\mathrm{AD}$ and $60 \%$ of the diploma school faculty members used CAI. Of the 9 administrators, 1 of the 3 from BSN schools (25\%) and all 5 from the AD schools used CAI. The single "computer lab personnel" employee who used CAI was from a BSN school. The findings concerning the types of institutions showed that $74 \%$ of the public and $57 \%$ of the private school faculty members used CAI. All 5 public school administrators used CAI and 1 administrator and 1 of the "computer lab personnel" from a private school were CAI-users. The findings showed that a larger percent of the faculty from large schools used CAI followed by small schools and lastly, by medium size schools. The exact percentages were $71 \%$ of the faculty from small schools, $63 \%$ from the medium and $83 \%$ from the large schools used CAI. Two of the 3 administrators from small schools, 1 of the 2 from medium size schools and 3 of the 4 from large schools used CAI. The single CAI-user "computer lab person”was from a medium size school.

The responses showed that 6 males and 102 females took part in the study and there was 1 non-response. All 4 of the male participants from AD schools used CAI and the 2 from BSN schools were both non-users. For the females, $57 \%$ of the BSN, $80 \%$ of the AD and $60 \%$ of the diploma school participants were CAI-users. When the findings were broken down by the type of institution, they showed that 4 of the 6 males from public schools used CAI. For the females, $72 \%$ 
of the public and $63 \%$ of the private school participants used CAI. An analysis by the size of school showed that 1 of the 2 men who worked for medium size schools and 3 of the 4 men who worked for large schools were CAI-users. A total of $80 \%$ of the female participants who used CAI worked for large schools $61 \%$ who worked for medium size schools and $72 \%$ who were from small schools used CAI.

Key Features of the Participants Who Use CAI

- Age Groups

* 20 to 40-years $(\mathrm{n}=12) \quad 7(58 \%)$ CAI-users

- 70\% AD, 0\% BSN and 0\% diploma

- $70 \%$ public and $0 \%$ private

- $75 \%$ small, $40 \%$ medium and $67 \%$ large

* 41 to 50-years $(\mathrm{n}=46) \quad 30(65 \%)$ CAI-users

- 50\% BSN, 78\% AD and 67\% diploma

- $68 \%$ public and $58 \%$ private

- 74\% small, $44 \%$ medium and $82 \%$ large

* 51 and over $(\mathrm{n}=50) \quad 37(74 \%)$ CAI-users

- $88 \% \mathrm{AD}$ and $59 \% \mathrm{BSN}$

- $74 \%$ public and $73 \%$ private

- $62 \%$ small, $77 \%$ medium and $80 \%$ large

An analysis of the age groups showed that there were no participants in the 20 to 40 -yearold age-group who worked for BSN or diploma schools but 7 of the 12 who worked for AD schools used CAI. In the 41 to 50 age-group, $50 \%$ of those from BSN schools used CAI, and $78 \%$ of the AD and $67 \%$ of the diploma school participants used CAI. For the 51 and above age-group, only $58 \%$ of the BSN and $88 \%$ of the AD participants used CAI. The findings concerning the 
participants based on the type of institution showed that for the 20 to 40 -year-old age-group, 7 of the 10 public school employees used CAI but none of the 3 from private schools in that age-group used it. For the 41 to 50 -year-old age-group, $68 \%$ of the public school and $58 \%$ of the private school employees were CAI-users. In the 50 and above age-group, $74 \%$ of the public and $73 \%$ of the private school employees used CAI. The analysis by the school sizes showed that for the 20 to 40-year-old age-group, 75\% from small schools, 40\% from medium and 67\% from large schools used CAI. For the 41 to 50-year-old age-group, 74\% from small, 44\% from the medium size and $82 \%$ from large schools were CAI-users. For the 50 and above age-group, $62 \%$ from the small, $77 \%$ of the medium and $80 \%$ of the large schools were CAI-users. 
Key Features of Participants Who Use CAI

- Educational Backgrounds

* BSN or lower $(n=7) \quad 6(86 \%)$ CAI-users

- All 5 AD and 1 (50\%) BSN

- 6 public and 0 private

- All 5 small and 1 large

* MSN (n=73) $46(63 \%)$ CAI-users

- 45\% BSN, 75\% AD and 100\% Diploma

- $65 \%$ public and $56 \%$ private

- $20(68 \%)$ small, $25(61 \%)$ medium and 1 (50\%) large

* Ph.D. $(\mathrm{n}=28) \quad 22$ (79\%) CAI-users

- $75 \% \mathrm{BSN}$ and $93 \% \mathrm{AD}$

- $81 \%$ public and $71 \%$ private

- $21(68 \%)$ large and 1 medium

The findings for the educational background of participants who used CAI showed that of the 2 participants with a bachelor degree or lower who were from BSN schools, 1 used CAI and the other did not. All 5 of the AD school employees who had a BSN degree or lower used CAI. The findings for participants with an MSN degree showed that $45 \%$ of the BSN, $75 \%$ of the AD and both, or $100 \%$ of the 2 diploma school participants used CAI. For the participants with a Ph.D., $75 \%$ of the BSN and $93 \%$ of the AD employees used CAI. Neither of the 2 diploma school participants with a Ph.D. used CAI.

When the same data was analyzed based on the type of institution, the findings showed that all 8 with a BSN or lower educational background were from pubic schools and of those, $88 \%$ used CAI. For the participants with an MSN degree, $65 \%$ of the public and $56 \%$ of the private school 
employees used CAI. Of the 28 participants with a Ph.D., 17 worked for public schools and of those, $81 \%$ used CAI. Of the 7 participants with a Ph.D. who worked for private schools, $71 \%$ used CAI. The analysis based on the school sizes showed that 5 of the 7 employees with a BSN degree or lower educational background worked for small schools and that $83 \%$ of those and 1 participant who worked for a large school used CAI. For the participants with an MSN degree, $67 \%$ of the CAI-users were from small schools, $61 \%$ were from medium size schools and only 1 , or $50 \%$ of those who were from large schools used CAI. Almost all, or 21 of the 28 participants with a Ph.D. worked for large schools and of those, $68 \%$ used CAI. Only 1 other participant with a Ph.D. who worked for a medium size school used CAI.

In summary, this section described the "key features" of the participants who used CAI. Previous findings from other sections of the study showed that $81 \%$ of the $\mathrm{AD}$ and $80 \%$ of the diploma schools used CAI as compared with only $56 \%$ of the participants from BSN schools. Those findings were confirmed by many of the findings in this section. Almost without exception, the findings showed that a larger percent of $\mathrm{AD}$ and diploma school participants used CAI than participants from BSN schools and that higher percentages of public school participants used CAI than those from private schools. The findings for the three different sizes of schools were mixed but participants from large schools usually used CAI more than those from small schools and with only a few exceptions, the number of CAI-users from the small schools were higher than the number from medium size schools.

Most of the participants were faculty members and almost three fourths of those and nearly three fourths of the administrators used CAI. It was surprising that only 1 of the 3 "computer lab personnel" participants used CAI. The percent of faculty from AD and diploma schools who used 
CAI was substantially higher than those from BSN schools. Likewise, only 1 of the 3 BSN administrators used CAI while all $5 \mathrm{AD}$ administrators were CAI-users. The single "computer lab personnel" participant who used CAI was from a BSN school. Almost three fourths of the faculty from public schools used CAI while just over half from private schools used it. All 5 public school administrators used CAI as did the single private school administrator and the single "computer lab personnel" from a private school. The percent of faculty who used CAI was highest for the large schools followed by small schools and was lowest for the medium size schools. The 2 administrators who worked for small and medium size schools used CAI as did 3 out of 4 who worked for large schools. The single computer "lab personnel"who used CAI was from a medium size school.

Four of the male participants from AD schools used CAI while the 2 from BSN schools did not. Fewer female participants from BSN schools used CAI than from either AD or diploma schools. All 6 males worked for public schools and of those, 4 used CAI. A larger percent of the females who worked for public schools used CAI than those who were from private schools. Of the 2 men who were from medium size schools, 1 used CAI and the other did not. Three of the 4 men who were from large schools used CAI. The highest percent of female participants who used CAI were from large schools followed by medium size schools and the lowest percent were from small schools.

Seven of the 12 participants in the 20 to 40 -year-old age-group who used CAI were from AD schools. A larger percent of the 41 to 50 -year and the 51 and over age-groups that used CAI were from AD and diploma schools than BSN schools. For the 20 to 40-year-old age-group, 7 of 10 public school participants used CAI but none of the private school participants did. For the 41 
to 50 age-group, the percent of public school participants who used CAI was $10 \%$ higher than for private schools but there was almost no difference in the 51 and older age-group. For the youngest age-group, the percent of participants who used CAI was highest for small schools followed by large schools. For the 41 to 50 age-group, most were from large schools followed by small. Large school participants who used CAI dominated in the 51-year and older age-group followed by medium.

A summary of the findings for the educational backgrounds of the participants showed that of the 2 participants with a BSN degree or lower who worked for BSN schools, only 1 used CAI while all 5 who worked for AD schools did. A larger percent of AD and diploma school participants with an MSN degree used CAI than those from BSN schools. The same was true of participants with a Ph.D. However, neither of the 2 from the diploma schools used CAI. In general, participants from public schools with all three levels of educational preparation used CAI more than their counterparts from private schools. Five of the participants with a BSN degree and lower from small schools and one from a large school were CAI-users. A larger number of participants with an MSN degree from small schools used CAI followed by medium size schools. Almost all of the participants with a Ph.D. worked for large schools and of those, over half used CAI. Only one other participant with a Ph.D. who worked for a medium size school used CAI. 
Key Features of Schools That Were Effective at Integrating CAI

- Computer lab in the SON (46 to 15)

- Computers in the SON (50 to 12)

- another department (44 to 17)

- students purchase their own computers (51 to 11)

- Computers in the library (15 to 48 )

- 16 or more computers in the SON (15 to 47)

- 31 or more in another department (23 to 35 )

- 25 or more in the library (19 to 39 )

- Technical and Instructional Support Personnel

- 3 or more technical (53 to 8 )

- 2 or more instructional (49 to 14)

- 4 or more technical and instructional (52 to 10)

- Percent of teachers that use CAI

- $41 \%$ or more of the teachers use CAI (35 to 27)

- $51 \%$ or more of the teachers used CAI (26 to 37)

\section{Key Features of Schools That Integrate Effectively}

The previous section focused on the key features of teachers who integrate CAI more often but this next section answered the second part of the third study question which asked about the "key features" of the schools that integrated CAI most effectively. The focus, was on some of the "key features" of the schools that use CAI most frequently and that rated their use as more effective when compared with others from the sample.

A basic assumption underlying this study was that teachers who used CAI were employed by schools that had more computer resources. A second was that those who use CAI more often would also have more occasions to use the integration strategies and as a result, would perceive 
them to be more "effective" than those who seldom used them. For that reason, in addition to identifying the obvious features, such as the types of schools, institutions and sizes of schools that use CAI, the focus of this portion of the study was on some of the less concrete features of the schools that were more effective at integrating CAI into their courses. Some of those features have already been discussed but were analyzed in this section differently to see how, or if, they impacted the way the participants used or perceived the "effectiveness" of the strategies. The features include things like the presence of a computer lab in the school of nursing, the locations and numbers of computers in the various departments, the types and numbers of computer support personnel and the percent of teachers at the school who use CAI. An analysis that consisted of calculating correlations using the Pearson $r$ test to compare the scores of two groups showed some outstanding differences but failed to show whether or not the strategies were "frequently" used or perceived to be "effective" by the participants. Therefore, the statistics that are discussed below consisted of dividing the sample into two groups, calculating the means for each strategy and then comparing to see which group had the most means that were higher. The findings of the calculations are included in Table 17.

The first comparison looked at the means of the 56 schools that had a computer lab within the school of nursing and compared them to 21 schools that used CAI but did not have a lab. The findings showed that schools with a lab had 46 means that were higher than schools that did not have a computer lab that only had 15 means that were higher. 
Table 17. Key Features of Schools: Comparison of the Means of the Integration Strategies $(n=77)$

\begin{tabular}{|c|c|c|c|c|c|c|c|}
\hline & & Frequency & Learning & Costs & Students' & Teachers' & Totals \\
\hline & & & & & Time & Time & \\
\hline Computer Lab in & $\begin{array}{l}\text { Lab in SON } \\
\quad(n=56)\end{array}$ & 17 & 13 & 5 & 4 & 7 & 46 \\
\hline \multirow[t]{2}{*}{ SON } & No Lab $(n=21)$ & 2 & 6 & 2 & 3 & 2 & 15 \\
\hline & No Difference & 0 & 0 & 1 & 0 & 1 & 2 \\
\hline Computers in & $\operatorname{SON}(n=65)$ & 17 & 19 & 2 & 7 & 5 & 50 \\
\hline \multirow[t]{2}{*}{ SON } & None $(n=12)$ & 2 & 0 & 6 & 0 & 4 & 12 \\
\hline & No Difference & 0 & 0 & 0 & 0 & 1 & 1 \\
\hline $\begin{array}{l}\text { Computers in } \\
\text { Another }\end{array}$ & $\begin{array}{l}\text { Another Dept } \\
\qquad(\mathrm{n}=49)\end{array}$ & 13 & 11 & 6 & 6 & 8 & 44 \\
\hline \multirow[t]{2}{*}{ Department } & None $(n=28)$ & 5 & 7 & 2 & 1 & 2 & 17 \\
\hline & No Difference & 1 & 1 & 0 & 0 & 0 & 2 \\
\hline \multirow[t]{3}{*}{$\begin{array}{l}\text { Computers in } \\
\text { Library }\end{array}$} & $\begin{array}{l}\text { Library } \\
(n=63)\end{array}$ & 9 & 4 & 0 & 2 & 0 & 15 \\
\hline & None $(n=14)$ & 10 & 15 & 8 & 5 & 10 & 48 \\
\hline & No Difference & 0 & 0 & 0 & 0 & 0 & 0 \\
\hline \multirow[t]{3}{*}{$\begin{array}{l}\text { Students } \\
\text { Purchase }\end{array}$} & $\begin{array}{l}\text { Purchase } \\
(n=43)\end{array}$ & 14 & 18 & 4 & 7 & 8 & 51 \\
\hline & $\begin{array}{l}\text { Do Not } \\
(n=34)\end{array}$ & 4 & 1 & 4 & 0 & 2 & 11 \\
\hline & No Difference & 1 & 0 & 0 & 0 & 0 & 1 \\
\hline
\end{tabular}


Table 17 (continued). Key Features of Schools: Comparison of the Means of the Integration Strategies $(n=77)$

\begin{tabular}{|c|c|c|c|c|c|c|c|}
\hline & & & & & Students' & Teachers' & \\
\hline & & Frequency & Learning & Costs & Time & Time & Totals \\
\hline Number of & 15 or $<(n=42)$ & 3 & 2 & 2 & 2 & 6 & 15 \\
\hline \multicolumn{8}{|l|}{ Computers in } \\
\hline \multirow[t]{2}{*}{ SON } & 16 or $>(n=35)$ & 16 & 17 & 5 & 5 & 4 & 47 \\
\hline & No Difference & 0 & 0 & 1 & 0 & 0 & 1 \\
\hline Number of & 30 or $<(n=45)$ & 3 & 10 & 3 & 2 & 5 & 23 \\
\hline \multicolumn{8}{|l|}{ Computers in } \\
\hline \multirow[t]{2}{*}{ Another Dept } & 31 or $>(n-32)$ & 15 & 8 & 2 & 5 & 5 & 35 \\
\hline & No Difference & 1 & 1 & 3 & 0 & 0 & 5 \\
\hline Number of & 24 or $<(n=45)$ & 3 & 7 & 3 & 0 & 6 & 19 \\
\hline \multicolumn{8}{|l|}{ Computers in } \\
\hline \multirow[t]{2}{*}{ Library } & 25 or $>(n=32)$ & 15 & 9 & 4 & 7 & 4 & 39 \\
\hline & No Difference & 1 & 3 & 1 & 0 & 0 & 5 \\
\hline Full \& Part-time & 2 or $<(n=44)$ & 1 & 1 & 2 & 1 & 3 & 8 \\
\hline \multicolumn{8}{|l|}{ Technical } \\
\hline Support & 3 or $>(n=33)$ & 18 & 17 & 6 & 5 & 7 & 53 \\
\hline \multicolumn{8}{|l|}{ Personnel } \\
\hline & No Difference & 0 & 1 & 0 & 1 & 0 & 2 \\
\hline Full \& Part-time & 1 or $<(n=45)$ & 2 & 0 & 5 & 1 & 6 & 14 \\
\hline \multicolumn{8}{|l|}{ Instructional } \\
\hline Support & 2 or $>(n=32)$ & 17 & 19 & 3 & 6 & 4 & 49 \\
\hline \multicolumn{8}{|l|}{ Personnel } \\
\hline & No Difference & 0 & 0 & 0 & 0 & 0 & 0 \\
\hline
\end{tabular}


Table 17 (continued). Key Features of Schools: A Comparison of the Means of the Integration Strategies $(n=77)$

\begin{tabular}{lccccccc}
\hline & & & & Students' & Teachers' \\
& & Frequency & Learning & Costs & Time & Time & Totals \\
\hline Full \& Part-time & 3 or $<(\mathrm{n}=38)$ & 3 & 1 & 2 & 1 & 3 & 10 \\
$\begin{array}{l}\text { Technical \& } \\
\text { Instructional }\end{array}$ & 4 or $>(\mathrm{n}=39)$ & 16 & 18 & 6 & 6 & 7 & 53 \\
$\begin{array}{l}\text { Support } \\
\text { Personnel }\end{array}$ & No Difference & 0 & 0 & 1 & 0 & 0 & 0
\end{tabular}

The next group of inquiries compared several parts of a single question that asked participants to indicate where the computers were located that were available for the students to use. The options included the school of nursing, another department, the library or students purchased their own and participants were asked to click on the radio button next to any or all that apply. The purpose of the comparison was not to compare schools that had computers with schools that did not, but rather to compare the different locations in order to see if any of the locations had the more or less impact on the frequency of use and perceived effectiveness of the strategies based on the participant's perceptions.

The first part of the question asked about the presence of computers in the school of nursing and the findings showed that the schools that had computers in the SON had an impressive 50 means that were higher than schools that did not. The next part of the same question asked about the presence of computers in another department of the school. The findings showed that 44 of the means were higher for the schools with computers in another department as compared with only 17 higher means for schools that did not. The next comparison yielded some surprising results 
because they showed that schools that had computers located in the library only had 15 means that were higher as compared with 48 means that were higher for schools that did not have computers in that location. The final part the question compared schools that had students purchase their own computers with those that did not. The findings showed that schools that had students purchase their own computers had 51 means that were higher and schools that did not only had 11 means that were higher.

The results seemed to indicate that the location of the computers was, in fact, related to the frequency of use and perception of the effectiveness of the integration strategies based on the participant's responses to questions 2 through 6 of the ICAI Questionniare. Those participants who had computers located in the SON, another department and had students purchase computers used the strategies much more "frequently" and perceived them to be more "effective" than participants from schools that did not. The meaning of the findings for the schools with computers located in the library is unclear and actually contradicts several other findings of this section of the study.

The next section looked at the number of computers located in each of three different departments to see if the location or the number of computers made a difference. The three locations included the school of nursing, another department and the library. The findings consistently showed that the schools with more computers used the strategies more "frequently" and perceived the strategies were more effective than schools with fewer computers. For the first comparison, schools with 16 or more computers located in the school of nursing had 47 means that were higher than schools that had 15 or fewer computers in that location. Schools with 15 or fewer computers only had 15 means that were higher. The second comparison was between the schools with 31 or more computers that were located in another department and those with 30 computers or 
less. The findings showed that schools with 31 or more computers had 35 means that were higher and schools with less than 30 computers had 23 means that were higher. The third comparison was between schools with 25 or more computers located in the library and schools with 24 or fewer computers. The findings showed that schools with 25 or more computers had 39 means that were higher as compared to 19 means that were higher for schools with less than 24 computers.

The findings indicated that both the locations of the computers and the number of computers were related to the frequency of use and perceived effectiveness of the integration strategies. More specifically, the findings suggest that schools that had even a smaller number of computers located in the school of nursing used the strategies much more often and perceive them to be more effective than schools with a larger number of computers that were located outside the department. They also seemed to suggest that fewer numbers of computers located in the library were associated with a positive impact on the frequency of use and perception of the effectiveness of the strategies than having a larger number of computers that were located in another department.

The next group of comparisons looked at the technical and instructional support personnel employed by the schools to see if those types of resources made a difference in the frequency of use and perceptions of effectiveness of the integration strategies in the minds of the participants. The first inquiry compared technical support personnel, the second looked at the number of instructional support personnel and the third combined the two. The findings showed that the schools that employed 3 or more technical support personnel had 53 means that were higher as compared to 8 means that were higher for schools with 2 or fewer technical employees. The findings of the second comparison showed that schools that employed 2 or more instructional support personnel had 49 means that were higher than schools that employed 1 or less. The third comparison 
combined technical and instructional support personnel and the findings showed that schools that employed 4 or more had 53 means that were higher and schools with 3 or less support personnel only had 10 means that were higher. The findings of all three parts of the question seemed to indicate that schools that the schools with higher numbers of technical or instruction support personnel used the integration strategies more "frequently" and perceived them to be more effective than schools with lower numbers of computer support personnel.

The next inquiry examined the percent of educators in the school of nursing that used CAI (See Table 18). The analyses consisted of two parts in which two different demarcation points were used to examine the same data to see if a small difference in the percent of teachers who used CAI made any difference in how "frequently" the integration strategies were used or how effective the participants perceived the strategies were. For the first analysis, the groups were divided into schools where $40 \%$ or below and $41 \%$ and above of the faculty used CAI. The demarcation point was selected so that the two groups were almost equal in number (38 and 39). The second analysis did not strive to divide the groups equally by number of participants but used a $50 \%$ or below and a $51 \%$ and above number as the cut-off point to compare the two groups. 
Table 18. Percent of Educators Who Use CAI: Comparison of the Means of the Integration Strategies $(n=77)$

\begin{tabular}{|c|c|c|c|c|c|c|c|}
\hline & & & & & Students' & Teachers' & \\
\hline & & Frequency & Learning & Costs & Time & Time & Totals \\
\hline \multirow{6}{*}{$\begin{array}{l}\text { Percent of } \\
\text { Educators that } \\
\text { Use CAI }\end{array}$} & $40 \%$ or $<$ & 11 & 15 & 4 & 1 & 4 & 35 \\
\hline & $(\mathrm{n}=39)$ & & & & & & \\
\hline & & & & & & & \\
\hline & $41 \%$ or $>$ & 8 & 4 & 4 & 6 & 5 & 27 \\
\hline & $(\mathrm{n}=38)$ & & & & & & \\
\hline & No difference & 0 & 0 & 0 & 0 & 1 & 1 \\
\hline \multirow{6}{*}{$\begin{array}{l}\text { Percent of } \\
\text { Educators that } \\
\text { Use CAI }\end{array}$} & $50 \%$ or $<$ & 9 & 4 & 4 & 1 & 8 & 26 \\
\hline & $(n=47)$ & & & & & & \\
\hline & & & & & & & \\
\hline & $51 \%$ or $>$ & 10 & 15 & 4 & 6 & 2 & 37 \\
\hline & $(\mathrm{n}=30)$ & & & & & & \\
\hline & No difference & 0 & 0 & 0 & 0 & 0 & 0 \\
\hline
\end{tabular}

The findings of the first analysis showed that schools where $41 \%$ or more of the teachers used CAI only had 27 means that were higher but the schools where $40 \%$ or fewer of the teachers used CAI actually had 35 means that were higher. Since the frequency of use was almost the same for both groups, the findings suggested the lower the percent of teachers who used CAI the more effective the participants perceived the strategies were. However, the findings of the second analysis showed an opposite trend in which schools where $50 \%$ or less of the teachers used CAI only had 26 means that were higher as compared to 37 higher means for the schools where $51 \%$ or more of the teachers used CAI. There were several points of interest noted in the findings. The 
first was that the means for the frequency of use and perceived effectiveness of the strategies to decrease costs and to decrease the students' time were essentially the same for both analyses. The largest differences when the data was analyzed the second time were in areas of the perceived effectiveness of the strategies to increase student learning and decrease the teachers' time. The findings seemed to suggest that even a $10 \%$ difference in the percent of teachers who used CAI made a large difference in how effective the participants perceived the strategies were for increasing learning and saving the teachers' time.

In summary, this portion of the study investigated the "key feature" of schools to determine which features had a positive impact on the frequency of use and perceptions of effectiveness of the integration strategies. The first comparison looked at schools that had a computer lab within the SON and the findings showed that schools with a lab used the strategies much more "frequently" and perceived the strategies to be more effective for each of the four purposes. The next batch of inquiries compared four different locations of computers. The findings for the first part showed that schools that had computers located in the school of nursing used more strategies and perceived them to be more effective for student learning, decreasing the students' time and the teachers' time but not for decreasing costs. The next analysis compared schools with computers in another department and the findings showed that schools with more computers used more strategies and rated them as more effective for all four purposes. The third inquiry looked at schools with computers in the library and the results were almost the reverse of the two previous findings. Schools that did not have computers in the library had a greater number of means that were higher for frequency of use and perceived effectiveness for all four purposes and had unanimous results for the strategies to decrease costs and decrease the teachers' time. The fourth part of the question 
compared schools where students purchased their own computers and the findings were in favor of schools where they purchased computers in all areas except costs where both groups had 4 means that were higher.

Another question examined the number of computers in three different departments to see if the number of computers or their locations made a difference. The findings for the first comparison showed that schools with more than 16 computers in the SON used the strategies more "frequently" and rated them higher in all areas, except decreasing the teachers' time. The findings for the second analysis showed that schools with 31 or more computers in another department also used the strategies more often and perceive them to be more effective for decreasing the students' and the teachers' time, but not for increasing learning or decreasing costs. The findings of the third analysis showed that schools with 25 or more computers in the library also had higher means for three purposes, but not for decreasing the teachers' time. In general, the findings showed that both the number of computers and their locations were important and were associated with increased frequency of use of the strategies and higher perceptions of effectiveness.

The next question looked at the number of full and part-time technical and instructional support personnel employed by the school. The findings for the first of the three studies showed that schools that employed 3 or more technical personnel had more means that were higher than schools with less than two. The findings of the second analysis found that schools that employed 2 or more instructional support personnel also had many more means that were higher than schools that employed fewer numbers. The third study combined the two types of support personnel and the findings showed that, once again, the schools with 3 or more technical and instructional support personnel had many more means that were higher than schools with fewer numbers of support 
personnel. Overall, the findings indicated that participants who worked for schools that employed larger numbers of technical and instructional support personnel used the integration strategies much more often and perceived them to be much more effective for all four purposes than teachers who worked at schools that employed fewer support personnel.

The final analysis in this section examined the percent of teachers in the school that used CAI to see if a small difference made a difference in the frequency of use and perceived effectiveness of the strategies. The findings of the first part of the analysis showed that the means for schools where $41 \%$ or above of the teachers used CAI were actually lower for the ratings of the effectiveness of the strategies than schools where less than $40 \%$ of the teachers used CAI. The second part of the study showed an almost opposite trend in which schools with $51 \%$ and above of the teachers used CAI had more means that were higher than schools with $50 \%$ and lower. The findings indicated that while the percent of faculty who used CAI had little bearing on the number of strategies the participants used or their perception of the effectiveness of the strategies for decreasing costs or decreasing the students' time, there was a positive difference in the way schools with $50 \%$ or more of the teachers who used CAI perceived the effectiveness of the strategies for increasing learning and decreasing the teachers' time. 
Five Types of Software

- 5,540 $(\mathrm{M}=50.82)$ programs used previous year

- $41 \%$ tutorials, $34 \%$ drill and practice, $19 \%$ simulations, $4 \%$ virtual reality and $2 \%$ expert systems

- Types of School

- AD 78\%, BSN $21 \%$ and diploma $0 \%$

- AD more D \& P, tutorials, and simulations; BSN tutorials

- Types of Institutions

- Public $94 \%$ and private $6 \%$

- Public and private mostly tutorials and drill \& practice

- Schools Sizes

- Large own 6 times small and 3 times medium

- Small mostly used tutorials and simulations

- Medium tutorials

- Large mostly used drill \& practice

\section{Study Question 4 Findings- Types of Software}

The fourth study question asked, "What types of CAI software programs are being used by nurse educators at your school to teach clinical decision-making?" Question 7 of the ICAI Questionnaire asked participants to type a number (0 to 999) to indicate how many of each of the five types of CAI software programs teachers at their school of nursing used to teach students clinical decision-making during the previous year. A discussion of the findings will follow. 


\section{Five Types of Software}

The data was analyzed and the findings showed that the109 sample schools reported they used a total of 5,540 programs $(\mathrm{M}=50.82)$ during the previous year. Of those, $41 \%$ were tutorial programs, 34\% were drill and practice, 19\% were simulations, 4\% were virtual reality programs and $2 \%$ were expert systems programs. An evaluation by the type of school showed that AD schools reported using all five types of software more often than bachelor or diploma schools (See Appendix Table C 61). In fact, AD schools used 78\%, BSN schools used 21\% and diploma schools used so few programs that it calculated to " $0 \%$ " of the total programs. A breakdown of the types of software that where used most showed that BSN schools used tutorials almost exclusively, or 70\% tutorials and AD schools used a slightly broader mix with 39\% drill and practice, 33\% tutorials and $21 \%$ simulations. Bachelor schools used $5 \%$ virtual reality programs while AD schools only used 3\%. On the other hand, AD schools also used 3\% expert systems software while BSN schools only reported using $2(0 \%)$ expert systems programs.

A second analysis sought to determine the types of institutions that used each of the five types of software. The findings showed that public schools used $94 \%$ and private schools only used $6 \%$ of the total software programs (See Appendix Table C 62). A breakdown of the types of software showed that public schools primarily used tutorials (41\%) and drill-and-practice programs $(34 \%)$ and the few programs that were used by private schools were mostly tutorials (46\%).

A third analysis was conducted to determine the types of software that were used by the three sizes of schools (See Appendix Table C 63). The findings showed that large schools used $66 \%$ of all the software programs, medium size schools used $23 \%$ and the small schools only used $11 \%$ of the total programs. A second analysis of the data showed that large schools used $43 \%$ drill- 
and-practice programs and 31\% tutorials. Medium size schools used $68 \%$ tutorials and small schools used $49 \%$ tutorials.

In summary, $\mathrm{AD}$ schools used three times as many software programs as the BSN schools and the number of programs used by the diploma schools were negligible. Of the small number of programs used by BSN schools, a large majority were tutorials. Associate schools used 39\% drilland-practice programs, $33 \%$ tutorials and $21 \%$ simulations. Public schools used over 17 times as many software programs as the private schools. Both public and private schools primarily used tutorials followed by drill-and-practice and a small number of simulations. Virtual reality programs held a slight lead over expert systems programs. Large schools used six times as many software programs as small schools and three times as many as medium size schools. The small and medium size schools primarily used tutorials while the large schools used more drill-and-practice programs followed by tutorials and a much small number of simulations. 
Commercially Purchased and In-house Produced Software

- 2,312 $(\mathrm{M}=21)$ programs

- $95 \%$ commercially purchased, $2 \%$ customized and $3 \%$ teacher-authored

- Types of Schools

- AD 56\%, BSN 43\% and diploma 1\%

- AD used more of all three than BSN schools

- AD more teacher-authored than customized and BSN more customized than teacher-authored

- Diploma only commercially-purchased

- Types of Institutions

- Public 10 times private

- Public commercially-purchased and small but equal numbers of customized and teacher-authored

- Private commercially purchased software

- School Sizes

- Medium twice as many as small

- Medium 1.7 times as many as large

- Small commercially-purchased and small number teacher-authored

- Medium commercially-purchased

- Large commercially-purchased and customized and teacher-authored 


\section{Commercially Produced and In-house Produced Software}

Question 9 of the ICAI Questionnaire asked participants to identify the number of commercially purchased and in-house produced software programs their schools used during the previous year. The findings showed that the schools used 2,312 total programs and of those, $95 \%$ were commercially-purchased, $2 \%$ were customized and $3 \%$ were teacher-authored programs. When the findings were broken down by the type of school they showed that AD schools used 56\%, BSN schools used $43 \%$ and diploma schools used $1 \%$ of the total (See Appendix Table C 64). As expected, the percentages for the 2,194 commercially-purchased programs were almost the same as for the total group. For the 55 customized software programs, BSN schools used $22 \%$ and AD schools used the other $78 \%$. For the teacher-authored software, AD schools used $66 \%$ and BSN schools used the other $33 \%$. A further breakdown of the same data showed that AD and BSN schools used $95 \%$ commercial software while the diploma schools only used commercially produced software. Bachelor schools used a larger number of custom-produced than teacherauthored software while AD schools used more teacher-authored programs than custom-produced.

A second analysis showed that public schools used $90 \%$ of the programs and private schools used the other 10\% (See Appendix Table C 65). Public schools used 94\% commercially purchased, $3 \%$ customized and 3\% teacher-authored programs. Private schools used $99 \%$ commerciallyproduced programs, no customized and $1 \%$ teacher-authored. A third analysis showed that medium schools used $47 \%$, large schools used $30 \%$ and small schools used $23 \%$ of the total programs (See Appendix Table C 66). A second breakdown of the data showed that small schools used $95 \%$ commercial, no customized and $4 \%$ teacher-authored programs. Medium size schools 
used $99 \%$ commercial software while large schools only used $88 \%$ commercially-purchased, $8 \%$ customized and $4 \%$ teacher-authored programs.

In summary, the second part of the fourth study question sought to determine the number of commercially purchased and in-house produced software programs used by the schools during the previous year. The findings showed that of the 2,312 programs that were used, almost all were commercially purchased programs. Associate schools only reported using $13 \%$ more of the programs than BSN schools in this part of the question but again, both schools used many more than diploma schools. While both AD and BSN schools reported using primarily commerciallypurchased programs, BSN schools used more customized than teacher-authored and AD schools used a higher number of teacher-authored programs than customized. Diploma schools used commercially purchased programs only. A summary of the findings for the types of institutions showed that public schools used nine times as many software programs as private schools. Public schools also reported using small but equal percentages of the customized and teacher-authored programs. The analysis by size of school showed that the medium sized schools used twice as many programs as the small schools and about one and a half times as many as the large schools. While all three used many more commercially-purchased programs, it was noted that small schools used a relatively large number of teacher-authored programs and large schools used almost all of the customized programs and an even large number of teacher-authored software than the small schools. Medium size schools almost exclusively used commercially purchased software. 
Ratings of Peer's Effectiveness at Integrating Five Types of Software

- Highest tutorials, simulations, D\&P, virtual reality \& expert systems

- Type of School

- AD more effective than BSN with all 5 types

- BSN best with simulations but used tutorials and D\&P more

- AD best with tutorials and used them most

- All thought expert systems least effectively integrated and used them least

- Type of Institution

- Public more effective than private with all 5 types

- Both best with tutorial and used them most

- Both least effective with expert system and used them least

- School Sizes

- Large more effective than small and small more effective than medium

- Large best with D\&P and used them most

- Medium best simulations but used tutorials and D\&P more

- Small best with tutorials and used them most

- All three least effective with expert systems

Study Question 5 Finding- Ratings of Peer's Effectiveness

The fifth study question asked, "How effectively do participants perceive teachers at their school have been at integrating the different types of CAI software designed to teach clinical decision-making into their courses." The discussion that follows will begin with the measures of central tendency that describe how effectively the participants perceived their peers were at integrating the five types of software and end with commercially-purchased and in-house produced 
software. That will be followed by a discussion of the correlations that related the frequency of use to the perceived effective at integrating the software into the courses.

Peer Ratings of Effectiveness of Peers at Integrating 5 Types of Software

As a follow-up to Question 7 on the ICAI Questionnaire, Question 8 asked participants to use a Likert rating scale (1 to 4) to indicate how strongly they "agreed" or "disagreed" with the statement that "teachers at their school were able to effectively integrate the five different types of software." The data was analyzed to determine which types of software participants perceived their peers were able to integrate most and least effectively. The findings for the entire group showed that the means for the tutorial programs $(\mathrm{M}=2.03)$ were highest, followed by simulations $(\mathrm{M}=$ 1.99), drill-and-practice $(\mathrm{M}=1.88)$, virtual reality $(\mathrm{M}=1.26)$, and finally, expert systems $(\mathrm{M}=$ $0.95)$.

Next, the measures of central tendency were calculated to determine how the different types of schools responded to the question (See Appendix Table C 67). Since the numbers of programs used by the diploma schools were negligible, the group was excluded from the discussion. A brief review of the findings showed that there was a similar pattern but that AD school participants rated their peers higher than BSN school participants at integrating all five types of software. One difference between the groups was that the AD school participants thought their peers were most effective at integrating tutorials, which was also the type of software they used most often and BSN schools rated their peers higher for integrating simulations, even though they used tutorials and drill-and-practice software more often. Both types of schools rated their peers as least effective at integrating expert systems, which was also the least often used type of program by both types of schools. 
A second analysis showed that public schools rated their peers as slightly higher than private schools at integrating all five software types . Both public and private school participants rated their peers as most effective at integrating tutorials, which was also the type of software both institutions used most often. Both public and private schools rated their peers as least effective at integrating expert systems software which was the type of software they used least often.

The findings of a third analysis showed that large school participants rated their peers highest followed by small school participants who were followed by medium size schools for all five types of software. Large school participants perceived their peers were most effective at integrating drill-and-practice programs, which were also the type of software they used most often. Small school participants rated their peers as most effective at integrating tutorial software which was also the type of software they used most and medium size schools rated simulations as highest even though they used tutorials and drill-and-practice software more often. Once again, all three size schools rated expert systems as the type of program their peers were least effective at integrating. Small and medium size schools reported using expert systems least often but the large schools used expert systems software slightly more often than they used virtual reality software. In summary, the findings showed that the entire group thought their peers were most effective at integrating tutorial software programs followed by simulations and least effective at integrating expert system software. A breakdown by the type of school showed that AD school participants rated their peers as more effective at integrating all five types of software than BSN participants. While BSN participants thought they integrated simulations best and AD participants thought they integrated tutorials best. Bachelor school participants used tutorials and drill-andpractice software more but $\mathrm{AD}$ participants used tutorials most. Both thought their peers were least 
effective at integrating expert systems into the course. Public school participants rated their peers slightly higher than private school participants at integrating all five types of software. Both thought they integrated tutorial programs best which was also the type of software they both used most and they rated themselves as least effective at integrating expert systems. The breakdown by the size of school showed that large schools rated their peers highest and medium size schools rated their peers as less effective than the other two. Large schools were most effective at integrating drill-and-practice software, medium schools were best with simulations and small schools were best at integrating tutorials. Medium sized schools actually used tutorial and drill-and-practice software more often than simulations but large and small schools thought their peers were most effective at integrating the types of software they used most. 
Peers' Effectiveness at Integrating Commercial or In-house Produced Software

- Most effective with commercially-purchased, teacher-authored and least with customized

- Types of Schools

- AD more effective than BSN all three

- AD and BSN more effective with commercial and least with customized

- Type of Institution

- Public more than private with all three

- Public and private more effective with commercial and least with customized

- Sizes of Schools

- Large more effective with all three

- Small more effective than medium with commercial and customized

- Medium more effective than small with teacher-authored

Peers' Rating of Effectiveness at Integrating Commercial and In-house Produced Software

Question 10 of the ICAI Questionnaire asked participants to rate their peers on effectiveness at integrating commercially-purchased or in-house produced software using a Likert scale (1 to 4) according to how strongly they agreed or disagreed with the statement: "teachers at your school of nursing are able to effectively integrate the following types of software to teach clinical decisionmaking into the courses they teach." The means for the entire group showed that not only did participants use commercially-purchased software much more "frequently" than the others, but that they also thought they were much more effective at integrating it. They also thought they 
integrated teacher-authored software more effectively than customized. The means were commercially-purchased $(\mathrm{M}=2.22)$, teacher-authored software $(\mathrm{M}=1.16)$ and customized $(\mathrm{M}=$ $1.02)$.

The findings of the analysis showed that $\mathrm{AD}$ participants rated their peers as more effective than BSN schools at integrating all three types of software (See Appendix Table C 68). Participants from AD and BSN schools rated their peers as most effective at integrating commercially-purchased and least effective at integrating customized software. The findings for the type of institution showed that public schools rated their peers as more effective than private schools at integrating all three types of software. Once again, commercial software was rated highest and customized was rated lowest by both. The findings for the different sizes of schools showed that large schools rated their peers as most effective at integrating all three types of software. Small school participants thought their peers were more effective than medium size schools at integrating commercially-purchased and customized, but medium size school participants rated their peers as slightly more effective than small school teachers at integrating teacherauthored software. 
Correlations Between Frequency of Use and Effectiveness of Peers at Integrating Software Five Types of Software

- Significant relationships for virtual reality and expert systems

- Type of School

- BSN and AD significant findings for virtual reality and expert systems

- BSN significant for drill-and-practice

- Type of Institution

- Public and private significant for virtual reality and expert systems

- Public significant for drill-and-practice

- School Sizes

- Small significant for virtual reality, expert systems and D\&P

- Medium significant for virtual reality and D\&P

- Large significant for all five types of software

Commercial and In-house Produced

- Entire group significant findings for customized and teacher-authored

- Type of School

- BSN significant for customized and teacher-authored

- AD significant for teacher-authored only

- Type of Institution

- Public significant for customized and teacher-authored

- Private significant for commercial and teacher-authored

- Size of Schools

- Small schools significant for all three

- Medium schools significant for teacher-authored

- Large schools significant for customized and teacher-authored 


\section{Correlations of Software}

Pearson $\mathrm{r}$ correlations were calculated to determine the relationship between the frequency of use and ratings of peer effectiveness at integrating the different types of software for the entire group $(\mathrm{N}=109)$. The findings showed significant relationships $(\mathrm{p}<0.01)$ for virtual reality $(\mathrm{r}=$ $0.53)$ and expert systems $(r=0.48)$ software. Once again, the strongest relationships were between the software that was not "frequently" used and was rated as least "effective."

A second correlation was performed and the findings showed that there was a significant relationship for both $\mathrm{BSN}(\mathrm{r}=0.58)$ and $\mathrm{AD}(\mathrm{r}=0.55)$ schools for virtual reality and for the $\mathrm{AD}(\mathrm{r}=0.54)$ and BSN $(\mathrm{r}=0.49)$ schools for expert systems and that there was a significant relationship for BSN schools only for drill-and-practice programs $(r=0.49)$.

A third correlation was calculated and the findings showed similarly significant relationships $(\mathrm{p}<0.01)$ for both public $(\mathrm{r}=0.57)$ and private schools $(\mathrm{r}=0.39)$ for virtual reality and for expert systems for public $(r=0.50)$ and private $(r=0.67)$ and for drill-and-practice programs for private schools only $(\mathrm{r}=0.40)$. A third correlation was calculated and showed there were significant relationships $(\mathrm{p}<0.01)$ for all five types of software for the large schools, and for virtual reality and drill-and-practice for the medium size schools and for virtual reality, expert systems and drill-and-practice for the small schools (See Table 19).

To summarize, it seemed participants from large schools used all five types of software more frequently and thought their peers did a better job of integrating it than any of the other groups examined. 
Table 19. Correlations between Frequency of Use and Ratings of Peer's Effectiveness at Integrating the Five Types of Software based on School Sizes $(\mathrm{N}=109)$

\begin{tabular}{llll}
\hline & Small & Medium & Large \\
\hline Simulation & -0.03 & 0.17 & $0.32 *$ \\
Tutorial & 0.26 & 0.01 & $0.43 *$ \\
Virtual Reality & $0.66^{*}$ & $0.41 *$ & $0.61 *$ \\
Expert System & $0.78^{*}$ & 0.23 & $0.67 *$ \\
Drill \& Practice & $0.35 *$ & $0.31 *$ & $0.31 *$
\end{tabular}

* Significant at 0.01 level of significance

Pearson $r$ correlations were also calculated to determine the relationship between the frequency of use and ratings of effectiveness for integrating commercially-purchased and in-house produced software. The findings for the entire group $(\mathrm{N}=109)$ showed there was a significant relationship for customized $(\mathrm{r}=0.37)$ and teacher-authored programs $(\mathrm{r}=0.47)$ at a 0.01 level of significance. Once again, the strongest significant relationships were between the types of software that were seldom used and those that were rated as least effective.

A second correlation was calculated and showed significant relationships for BSN schools for customized $(\mathrm{r}=0.59)$ and teacher-authored $(\mathrm{r}=0.47)$ programs and for AD schools for the teacher-authored software $(\mathrm{r}=0.41)$ only. The third correlation showed significant relationships for public schools for customized $(r=0.39)$ and teacher-authored $(r=0.44)$ and for private schools for commercial $(\mathrm{r}=0.38)$ and teacher-authored $(\mathrm{r}=0.52)$. The final correlation that was based on the school size showed that large schools had significant relationships for customized $(r=0.43)$ and 
teacher-authored software $(\mathrm{r}=0.42)$, medium size schools had significant relationships for teacherauthored $(\mathrm{r}=0.50)$ and small schools had significant relationships for commercially produced $(\mathrm{r}=$ $0.39)$, customized $(r=0.57)$ and teacher-authored $(r=0.39)$ software.

In summary, the findings showed that not only were commercially-purchased software programs used much more frequently than the other two but participants also thought their peers were much more effective at integrating it than the other two much more expensive alternatives. They also rated their peers as more effective at integrating teacher-authored software than customproduced software. As before, $\mathrm{AD}$ school participants rated their peers as more effective at integrating all three types of software than BSN teachers and both groups thought they integrated commercially-purchased software best and custom-produced least effectively. The comparison of the two types of institutions showed that public schools integrated all three types more effectively than private schools and commercially-purchased software was integrated more effectively by both public and private teachers. The analysis by sizes of schools showed that large schools rated their peers as most effective at integrating all three types of software followed by small schools that were rated higher at integrating commercially-purchased and customized. Participants from medium size schools rated their peers as better at integrating teacher-authored software than small schools.

Pearson $r$ correlations were calculated to determine the strength of the relationships between the frequency of use and ratings of effectiveness of the 5 different types of software and the findings for the entire group showed statistically significant relationships $(p<0.01)$ for virtual reality and expert systems software. The findings for the two types of schools were the same, except that BSN schools also had a significant relationship for drill-and-practice software. The same results, significant relationships for virtual reality and expert systems, were obtained for 
public and private schools with private schools also having a significant relationship for drill-andpractice software. The correlations for the three different sizes of schools showed that large schools had significant relationships for all five types of software, medium size schools had significant relationships for virtual reality and drill-and-practice programs and for the small schools, the relationships were significant for virtual reality, expert systems and drill-and-practice software. Correlations were also calculated for the commercially-purchased and in-house produced software for the entire group and the findings showed that there was a significant relationship for customized and teacher-authored software. A correlation to compare the two types of schools showed that BSN schools had significant relationships for customized and teacher-authored software and AD schools for teacher-authored software only. Another correlation based on type of institution showed there were significant relationships for public schools for customized and teacher-authored software and private schools for commercially-purchased and teacher-authored software. The correlation based on the school size showed significant relationships for large schools for customized and teacher-authored, medium size schools for teacher-authored only and small schools for commercially-produced, customized and teacher-authored. 


\section{CHAPTER V}

\section{SUMMARY AND CONCLUSIONS}

As stated previously, there are four reasons teachers use integration strategies. They are to increase student learning, decrease course costs, decrease the students' or the teachers' time. Historically, teachers have primary been concerned with student learning and it has remained the sole determining factor behind almost every decision the teacher made. More recently, economic and political pressures are pushing educational productivity issues to the forefront so that teachers can simply no longer ignore the other three factors as if they are not also important components of good teaching. One of the primary goals of this study was to begin exploring, almost in a pioneering fashion, the thoughts, practices and values of educators who are beginning to make the paradigm shift as they use new techniques to prepare students who must be better prepared than ever before to make decisions in the clinical setting.

As an aid to the reader, this final chapter restates the research problem and reviews the major methods used in the study. The major sections of this chapter summarize the results and discuss their implications as they relate to the literature.

\section{Review of the Study}

\section{The Problem Statement}

The problem was nurse educators did not know which integration strategies were being used or which ones were perceived by participants from the area of nursing education as effective for increasing student learning, reducing course costs, or the students' time, or the teachers' time. Nurse educators also needed to know the characteristics of schools and teachers that were perceived by participants as integrating CAI effectively, the types of CAI software that 
were being used to teach clinical decision-making, the types of in-house or commerciallyproduced programs that were being used and which types of software participants perceived their peers were able to integrate into their courses effectively.

\section{The Methodology}

As explained in Chapter 3, the study reported here was a descriptive study design that used a stratified randomly selected sample of representatives from 109 schools of nursing from across the United States. The sample was a statistically proportionate representative mix of BSN, AD, and diploma schools from six geographic regions of the United States. The schools were randomly selected from a list of all of the 1,398 schools of nursing in the United States until the target number of 37\% bachelor, $58 \%$ associate and 5\% diploma schools from each of the six geographic regions were contacted. The target number of 140 schools was $10 \%$ of the total population of schools.

The actual number of participants who completed the questionnaire represented 109 schools, and the response rate for the study was $78 \%$. The sample population represented the proportionate mix of $\mathrm{BSN}, \mathrm{AD}$ and diploma schools from each geographic region closely but was a little lower than the target number in each of the six regions. The target number was exceeded by four bachelor schools and was 4 less than the target number for AD schools. The "Integration of CAI Questionnaire," an on-line electronic survey designed by the investigator, was used to collect data. It was comprised of a list of 19 integration strategies that were gleaned from a review of the literature. The on-line electronic questionnaire took participants approximately 20 minutes to complete and once finished, participants simply clicked on a radio button to return the parsed data directly to the researcher's Internet e-mail address. 
The statistical analysis consisted of measures of central tendency, frequencies, and percentages that were calculated with a data-processing software program entitled, "Corel Quattro Pro." The Pearson product-moment correlation coefficient was used to determine the relationships between the frequency of use and perceptions of the effectiveness of each strategy. Means were calculated and the totals were compared in order to identify relationships between descriptive demographic variables (type of schools, computer resources, and other key features of the schools) and the frequency of use and perceived effectiveness of the integration strategies. The numbers and locations of computers within the school, computer support personnel and percent of teachers from the school that used CAI were also calculated. Other statistics included the types of software the schools used to teach clinical decision-making, the number of commercially-purchased or in-house produced software programs, and the participant's perceptions of the effectiveness of their peers at integrating the various types of software into the courses they taught.

\section{Summary of the Results}

\section{Description of the Sample}

The sample was comprised of 109 participants who represented 45 bachelors, 59 associate and 5 diploma schools. They consisted of 84 public schools and of those, 53 were AD schools and 30 were from BSN schools. The 25 private schools were made up of $15 \mathrm{BSN}$ and 6 AD schools. One of the diploma schools was a public school and the other 4 were private. Associate schools made up $64 \%$ of the small schools, $50 \%$ of the medium and $48 \%$ of the large 
Integration of CAI 267

schools. Bachelor schools comprised $31 \%$ of the small schools, $48 \%$ of the medium and $45 \%$ of the large schools and diploma schools made up $6 \%$ of the small, $2 \%$ of the medium and $7 \%$ of the large schools.

A majority of the schools in the sample, or $71 \%$ used CAI and $29 \%$ were non-users according to responses to the first question. More AD and diploma schools used CAI than BSN schools, public schools used it more than private and large schools used it more than medium or small. More specifically, of the CAI-users, $62 \%$ were AD and only $32 \%$ were BSN schools. Non-users were made up of $62 \%$ BSN schools and 34\% AD schools. The same data viewed differently showed that $56 \%$ of all BSN schools, $82 \%$ of all AD and $80 \%$ of all diploma schools in the sample used CAI. When the CAI-user schools were broken down by the types of institutions, the findings showed that $55 \%$ of the public schools and only $16 \%$ of the CAI-users were private schools. Among the non-users, $22 \%$ were public and $7 \%$ were private. When the same data was examined from another perspective, it showed that $71 \%$ of all public schools used CAI and $68 \%$ of all private schools used it. When the CAI- user schools were broken down by sizes, $23 \%$ were small schools, $26 \%$ were medium and $22 \%$ were large schools. Among the nonusers, $10 \%$ were small schools, $15 \%$ were medium, and 5\% were large schools. Viewed from another perspective, the findings showed that $83 \%$ of all large, $64 \%$ of all medium and $63 \%$ of all small schools used CAI. A closer examination showed that $57 \%$ of the medium BSN schools were CAI-users as compared with $45 \%$ of the large BSN and $38 \%$ of the small BSN schools. Conversely, $83 \%$ of the small AD schools were CAI-users as compared to $68 \%$ of the medium and only $24 \%$ of the large AD schools. 
Integration of CAI 268

\section{Discussion of the findings}

Frequently used strategies. The findings of the first study question showed that 29 out of 44 , or $66 \%$ of the strategies were "frequently" used by the sample group. Eleven of the 19 integration strategies, or $58 \%$ to increase learning were "frequently" used. Four of the conceptually similar preinstruction strategies "explain CAI, explain objectives, motivate and integrate" were used "frequently" but the "pretest" strategy was not. The 3 mid-instruction strategies that were "frequently" used included "technical" and "instructional" support and the "individual" mode of instruction. The other 2 modes of instruction "small-group" and "wholeclass" were seldom used. The post-instruction strategies that were "frequently" used were "required, follow-up, course exam, and post-instruction." The "optional"and "replace traditional" strategies were seldom used. Including CAI content as part of the "course exam" was "frequently" used but the use of "embedded" tests was not. While the "follow-up" and "post-instruction" strategies were "frequently" used, providing "alternative instruction" for learners who fail to meet the course objectives using the CAI was not. The strategy of "evaluating CAI" was not "frequently" used but the item-by-item analysis showed that it was actually used much more often than the "pretest" strategy.

Four of the 8 , or $50 \%$ of the strategies to decrease course costs were "frequently" used. They included providing "technical" and "instructional" support, an "individual" mode of instruction and assigning CAI as "required" part of the course. For the strategies to decrease the student's time, 5 of the 7, or 71\% were "frequently" used. They included "explain CAI" and "explain objectives" and providing "technical" and "instructional" support and the "individual" mode. Four of the 10 , or $40 \%$ of the strategies to decrease the teachers' time were "frequently" 
used. They included an "individual" mode of instruction, "technical and instructional" support and "course-exam."

In general, the number of strategies that were perceive to be "effective" were far fewer than those that were "frequently" used. In all, only 17 of the 44 , or $38 \%$ of the strategies were perceived to be "effective" as compared to the 29 of the 44 , or $66 \%$ that were "frequently" used. It was noted however, that the drop was only in the areas of costs, students' and the teachers' time and not in the area of student learning.

Effective strategies. In fact, the exact same strategies that were above 2.50 for "frequency" of use were also above a mean of 3.00 for perceived "effectiveness"for student learning. Once again, the figure was 11 strategies out of 19 , or $58 \%$ that were perceived of as "effective" for increasing student learning. They included the four conceptually similar preinstruction strategies but, once again, the "pretest"strategy was not. The three mid-instruction strategies of providing "technical" and "instructional" support and an "individual" mode of instruction were above 3.00 while the "small-group" and "whole-class"strategies were not. While the post-instruction strategies were ranked differently, the same four were above 3.00 for effectiveness as were "frequently" used. Participants indicated they perceived designating a CAI assignment as "required" was more effective than designating it as an "optional" assignment. However, they did not perceive using CAI to "replace traditional" instruction as "effective" for increasing student learning. The two post-instruction strategies of engaging students in a "postinstruction" discussion or critique and providing "follow-up" activities were perceived to be effective strategies for increasing learning but providing "alternative" assignments was not. 
Participants also thought including the content of the CAI on an in-class "course exam" was effective but the use of "embedded" tests was not.

The findings for the effectiveness of the strategies for decreasing the course costs showed that the participants did not perceive any of the strategies were "effective" for reducing costs. The analysis of the item-by-item percentages of the responses showed that a majority did perceive using CAI to "replace traditional" instruction did decrease costs. Also, while a majority thought designating CAI as a "required" assignment was not an effective way to reduce costs, the number that thought making it an "optional" assignment was much lower.

There were 3 strategies out of 7 , or $43 \%$ that were perceived to be "effective" for reducing the students' time. They included providing "technical" and "instructional" support and the preinstruction strategy, "explain CAI." The other strategies of using an "individualized" mode of instruction as well as "small group" and "whole class" mode were not perceived to be effective strategies for decreasing the students' time. Another 3 of 10 strategies, or 30\% were perceived to be effective for decreasing the teachers' time. They included providing "technical" and "instructional" support and the use of an "individual" mode of instruction. None of the others, such as using CAI to "replace traditional" instruction, including CAI content on "course exams" or the use of "embedded" test grades were perceived be effective strategies for saving the teachers' time.

Significant correlations. The findings of the correlations of the entire sample showed that there were 15 statistically significant findings for the strategies to increase student learning. Seven that were also "frequently" used and perceived to be effective included "explain CAI, explain objectives, integrate, motivate, individual mode, course exam, and follow-up." There 
were 2 significant strategies for decreasing course costs, 2 that were significant for decreasing the students' time and 2 for decreasing the teachers' time but none met the other criteria of also being "frequently" used and "effective."

Comparison of strategies. The first analysis in the next section compared the means of the three types of schools for the "frequency" of use of the strategies. The findings showed that BSN schools used 36 (81\%) of strategies, AD schools used 28 (63\%) and diploma schools used $22(50 \%)$ of the strategies "frequently." A breakdown of the statistics by each of the four purposes showed that BSN schools used the strategies to decrease the students' time, costs and the teachers' time much more often than AD schools. For the strategies to increase student learning, BSN schools used 14 of the 19 strategies, or 73\%, while AD schools used 12, or $63 \%$ and diploma schools used 9 , or $47 \%$ of the strategies "frequently." For the strategies to decrease costs, BSN schools used 7, or $88 \%$, while AD schools used 5, or 63\% "frequently." For the strategies to decrease the students' time, BSN schools used all 7, or 100\%, while AD schools used 5 , or $71 \%$ and diploma schools used 3 , or $43 \%$ of the strategies "frequently." For the strategies to decrease the teachers' time, BSN schools used 8 of the 10 strategies, or $80 \%$, while AD schools used 6, or $60 \%$ and diploma schools used 5, or $50 \%$ of the strategies "frequently." To summarize, BSN school participants used several more strategies for each of the four purposes than AD schools and those strategies were the use of "small-group, and whole-class modes of learning and replace traditional instruction."

A comparison of public and private schools showed that public schools used 29, or $66 \%$, and public schools used 23 , or $52 \%$ of the strategies "frequently." A comparison of the three sizes of schools showed that medium schools used 34 , or $77 \%$, large schools used 32 , or 
$73 \%$ of the strategies, while small schools only used 19 , or $43 \%$ of the 44 strategies "frequently." The patterns of use were similar among the three sizes of schools but the means for small schools were lower.

The findings of the next comparison that looked at the perceived "effectiveness" of the strategies for increasing student learning showed that BSN schools perceived 13 , or $68 \%$ of the strategies, while AD schools rated 12 , or $63 \%$ and diploma schools did not rate any strategies as "effective." As with the entire group, the strategies that were perceived to be most "effective" were also the ones that were used most "frequently" by each of the schools. In fact, BSN schools had 12 strategies that were both "frequently" used and perceived to be "effective" and AD schools had 11 strategies that met both criteria.

The findings of "effectiveness" based on the type of institution showed that public schools rated 12 strategies, or $63 \%$ and private schools rated 9 strategies, or $47 \%$ of the strategies as "effective." Public schools indicated 11 strategies and private schools indicated 8 strategies were both "frequently" used and "effective." The comparison based on the three school sizes showed that medium and large schools rated 13 , or $68 \%$, while small schools only rated 8 , or $42 \%$ of the strategies as "effective." Medium and large size schools both had 12 strategies that were both "effective" and "frequently" used while small schools only had seven strategies that met both criteria.

The findings concerning the perceived "effectiveness" of the strategies for decreasing costs showed that as with the entire group none of the strategies had a mean above 3.00 and therefore, none were perceived to be "effective" for decreasing costs. In general, there were few differences between BSN and AD schools but the means for the diploma schools were much 
lower that the other two. A comparison of means for public and private institutions showed, once again, that public schools had higher means for all eight strategies. Some of the unusual findings that were noted were that while private schools ranked the strategy of "replace traditional" instruction as higher than the others, they seldom used the strategy. Public schools rated the provision of "technical" and "instructional" support as two of the highest while private schools ranked them near the bottom, despite the fact that they often used the two strategies. The third analysis based on the school's sizes showed that small schools perceived the strategies were less "effective" than medium size schools and medium size schools perceived the strategies were less "effective" than large schools. Overall, the means for the three sizes of schools were similar despite the large differences in the resources of the schools.

The comparison of the "effectiveness" of the strategies for decreasing the students' time showed that both BSN and AD schools rated 4, or $57 \%$ of the strategies as "effective" but the diploma schools did not think any of the strategies were. The strategies that were perceived of as most "effective" for decreasing the students" time were "technical" and "instructional support."

The findings of the comparison of the two types of institutions showed that public schools rated 4 , or $57 \%$ of the strategies as effective while private schools rated 3 , or $42 \%$ of the strategies as "effective." A comparison of the three sizes of schools showed that large and medium size schools rated 5 , or $71 \%$ of the strategies as effective, while small schools only rated 3 , or $42 \%$ of the strategies as "effective." Once again, the strategies of providing "technical" and "instructional support" were perceived as the most "effective."

A comparison of the effectiveness of the strategies for decreasing the teachers' time 
showed that BSN schools rated 4 , or $40 \%$ and $\mathrm{AD}$ schools rated 3 , or $30 \%$ of the strategies as effective while diploma schools did not think any were "effective." The patterns of the strategies BSN and AD schools perceived as most and least effective differed strikingly from the strategies they thought were effective for reducing the students' time. The two schools tended to agree on the strategies they perceived to be most effective but disagreed on the ones that were not. Bachelor schools thought including questions from the CAI on "course exams" was a time-saver for the teacher but $\mathrm{AD}$ and diploma schools did not. None of the schools thought the strategy, "replace traditional" instruction was an effective way to save the teacher time but they perceived the use of "individualized" instruction was one of the most "effective." The analysis based on the type of institution showed that public schools rated 3, or 30\% and private schools rated 2, or $20 \%$ of the strategies as "effective." Public schools thought the strategy of "replace traditional" instruction was more "effective" than private schools. The comparison based on the sizes of schools showed that medium size schools perceived 4 , or $40 \%$, and large schools rated 2 , or $20 \%$ of the strategies as effective while small schools did not think any were "effective" for decreasing the teachers' time. Once again, the strategies of provide "technical" and "instructional support" and an "individual" mode were thought to be the three most "effective" strategies for decreasing the teacher's time.

Many of the strategies for increasing student learning were statistically significance $(\mathrm{p}<$ 0.01 ), however, only those that also met the criteria of a mean of 2.50 for "frequency" and 3.00 for "effectiveness" were included in the discussion since they were the only ones that were of interest to this study.

The findings of the first analysis showed that BSN and AD schools both had 7 strategies 
that were statistically significant and met the other two criteria. Those for the BSN schools included the 4 conceptually similar preinstruction strategies (explain objectives, explain CAI, motivate and integrate), "individual mode," "required" and "course-exam." The 7 strategies for the AD schools included the four preinstruction strategies, "technical support," and "course exam," and "follow-up." The findings of a comparison based on the type of institution showed that public schools had 6 significant correlations and they included the four preinstruction strategies and "course-exam" and "follow-up." Private schools had 5 significant strategies that included the four preinstruction strategies and an "individual mode" of instruction. The correlations for the sizes of schools showed that small schools had 6, medium schools only had 3 and large schools had 8 significant correlations. The strategies for small schools included three of the preinstruction strategies "explain CAI, motivate, and integrate," an "individual” mode, "instructional support," and "required." Significant correlations for the medium size schools included "individual," and "small group" mode, and "course-exam." The significant correlations for the large schools included the four preinstruction strategies and "course-exam," "follow-up," and "post-instruction."

The findings for significant correlations of the strategies to decrease costs showed that even though there were several statistically significant relationships, since none of the strategies met the criteria of being "effective," even those that were "frequently" used failed to meet both criteria and were excluded. There were 4 statistically significant findings for the BSN schools and even though they were all "frequently" used, none met the criteria for "effectiveness." The same was true for the findings of several strategies that were significant and "frequently" used but were not "effective" for public and private and the three sizes of schools. 
The findings of the correlations of the strategies to reduce the students' time showed that “explain CAI" was significant for BSN schools as was "explain objectives" for the AD schools. Public schools had none and private schools had a significant relationship for "explain CAI." The strategy, "explain CAI" was statistically significant for small schools, and medium size schools had a significant negative correlation for the strategy of "instructional support," and large schools had none. A summary of the findings for the strategies to decrease the students' time showed that the strategy " explain CAI" was identified as significant for BSN, private and small schools, "explain objectives" met all three criteria for AD schools and "instructional" support met the criteria for medium size schools.

The correlation study of strategies to decrease the teachers' time showed that 'individual mode" of instruction was the single strategy that was statistically significant for the BSN schools. None of the other significant correlations met the criteria for "frequency" of use or "effectiveness" for public and private schools or for the three sizes of schools.

Computer resources. The findings of the inquiry into whether or not the schools had a computer lab in the school of nursing showed that over twice as many schools had a lab as did not. More specifically, $69 \%$ had a lab and 30\% did not. A breakdown of the findings showed that $68 \%$ of the $\mathrm{AD}$ and $68 \%$ of the $\mathrm{BSN}$ schools and all five, or $100 \%$ of the diploma schools had a lab. A total of $71 \%$ of the public schools had a lab and $64 \%$ of the private schools did. When the data was examined based on the school size, it showed that $68 \%$ of the small, $60 \%$ of the medium size schools and $83 \%$ of the large schools had a computer lab in the school of nursing. 
The next part of the study compared the four different locations of the computers students used and the findings showed that out of a total of 303 responses, $29 \%$ indicated they had computers in the SON, $29 \%$ had them in the library, $21 \%$ had computers in another department, and another $21 \%$ had students purchase their own.

In all, $72 \%$ of the respondents to the question used CAI and $28 \%$ did not. Schools that used CAI indicated they had computers located in the SON and non-users said they had computers located in the library and had students purchase their own computers more often. A breakdown of the locations of the computers showed that BSN schools had the highest percent of their computers in the library followed by the SON and they also had students purchase their own computers more often than AD or diploma schools. Associate schools had equal numbers of computers in the library and the SON and had students purchase their own computers less than the others. Diploma schools had $40 \%$ of their computers located in library and the school of nursing and only $10 \%$ in each of the other two locations. A large majority of the responses were from public schools who had the highest percent located in the SON and then the library. Most of the computers in the private schools were located in the library followed by having students purchasing their own. Medium size school reported more computers than large or small. The highest percent of computers in small and medium size schools were in the library but large schools had a higher percent in the SON.

The findings to another, similar question asked participants to identify the number of computers that were available for students to use that were located in each of three locations. The findings showed that the combined sample schools reported owning a total of 12,265 computers, or an average of 113. 
The findings showed that CAI-user schools owned three times as many computers that were located in the "SON" and twice as many that were located in the "library" and "another department" as non-user schools. Bachelor schools owned 55\%, AD schools owned 44\% and the other $1 \%$ were in diploma schools. The means for the three types of schools showed that BSN schools had a mean of 52, AD had a mean of 31 and diploma schools had a mean of 9 computers. The majority of the computers in BSN schools were located in the library and another department and only $11 \%$ were in the school of nursing. Associate schools had most of theirs in another department and $17 \%$ in the school of nursing. Diploma schools reported the majority of their computers were in the school of nursing.

Public schools owned $87 \%$ and private schools only owned $13 \%$ of the total computers. Most of the computers for public schools were located in another department with a small percent in the SON. Surprisingly, medium size schools owned 46\%, small schools owned $26 \%$ and large schools owned $29 \%$ of the total computers. Small schools reported most of their computers were in another department, medium schools had equal numbers in another department and the library, and large schools had more computers in another department, but they also had a larger number in the school of nursing than the others.

The findings concerning the number of full and part-time technical and instructional support personnel were reported next and showed that a total of 278 full-time technical (FTT), 191 part-time technical (PTT), 138 full-time instructional (FTI) and 85 part-time instructional (PTI) personnel were employed by the sample schools. The findings of a comparison of the number of support personnel employed showed that CAI-user schools employed 5 times as many full and part-time technical and 9 times as many instructional support personnel as non-user 
schools. CAI-user schools also had a higher ratio of full-to-part-time employees for both technical and instructional support personnel.

Human resources. Associate schools employed 25\% more full and part-time technical support personnel than BSN schools and 61\% more than diploma schools. Associate schools also employed $15 \%$ more full and part-time instructional support personnel than BSN schools but the BSN schools had a higher ratio of full-to-part-time technical and instructional support personnel. The analysis by the type of institution showed that public schools employed 5 times as many technical and 3 times as many instructional support personnel as private schools. Public and private schools had the exact same ratio of full-to-part-time technical support personnel but public schools had a higher ratio of full-to-part-time instructional support personnel than private schools. The findings based on the size of the schools showed that large schools had more fulltime technical and small schools employed more part-time technical. Medium size schools employed more full-time instructional support personnel and large schools employed the most part-time instructional support personnel. Medium schools had the highest ratio of full-to-parttime technical support personnel and small schools had the highest ratio of full-to-part-time instructional support personnel.

The next part of the study investigated the number of teachers from sample schools that used CAI and what percent of the faculty at the school of nursing that number represented. The findings for the first part of the question showed that a total of 648 faculty members at the participants' schools used CAI. The responses to this question differed slightly from the responses to the first question in that more teachers from $\mathrm{AD}$, public and large, instead of medium size schools used CAI. More precisely, the responses to this question showed that 56\% 
from AD schools, 38\% from BSN and 6\% from diploma schools used CAI. The analysis by type of institution showed that $79 \%$ of the CAI-users worked for public schools and $21 \%$ for private institutions. The findings for the size of school showed $45 \%$ of the CAI-users were employed by large schools, $31 \%$ were from medium sized schools and $24 \%$ were from small schools.

The responses to part " $\mathrm{B}$ " of the question that asked participants to indicate what percent of faculty at their schools used CAI were placed in a frequency distribution table and chart that showed that 28 responders indicated " $0 \%$ " of the faculty at their school used CAI, 50 responders said somewhere between $1 \%$ and $50 \%$ of the faculty at their school used CAI and when the numbers were combined, they showed that 78 responders said that less than $50 \%$ of the faculty at their schools used CAI. Another $25 \%$ at the other end said somewhere between $80 \%$ and $100 \%$ of the faculty at their school used CAI.

When the data was broken down for comparison, the findings showed that $17 \mathrm{BSN}$ and 10 respondents from AD schools said " $0 \%$ " of the faculty at their schools used CAI. Associate degree schools had 27 respondents who said $51 \%$ or more of the teachers at their schools used CAI while BSN schools only had 3 respondents with that number. When the data was broken down by the type of institution, the findings showed that public schools strongly outnumbered private schools for the number of " $0 \%$ " responses. Public schools also had 26 respondents who indicated $51 \%$ or above of the faculty at their school used CAI. A third analysis by the school size showed that small schools had 17 respondents who indicated that $51 \%$ or more of the teachers used CAI while medium and large schools only had 6 responses of $51 \%$ or above.

The purpose of the next section was to describe the key features of the participants who 
indicated that they used CAI. The analysis that compared the roles of participants showed that 97 of the participants were faculty members, 9 were administrators and 3 were computer lab employees. A total of $72 \%$ of the faculty members and $67 \%$ of the administrators used CAI. Only 1 of the 3 computer lab personnel participants used CAI.

A breakdown by the type of school showed $81 \%$ of the AD, $80 \%$ of the diploma and $56 \%$ of all of the BSN faculty member participants were CAI-users. A breakdown by the type of institution showed that $74 \%$ of all public and $57 \%$ of all private school faculty members used CAI. The analysis by the size of school showed that $71 \%$ of the small, $63 \%$ of the medium and $83 \%$ of the large school faculty members used CAI. Among the 6 administrators who used CAI, 5 were from AD schools and 1 was from a BSN school. Similarly, 5 administrators who used CAI were from public schools and only 1was from a private school. The single computer lab employee who was a CAI-user was from a medium sized private BSN school. A summary of the findings for the role of participants who used CAI showed that faculty members from large, public AD schools were the largest CAI-users. For the administrators, those who worked at large, public AD schools used CAI most often.

An analysis of the participants' genders showed that $60 \%$ of the males and $70 \%$ of the female participants were CAI-users. All 4 of the males that used were from AD schools and the 2 non-users were both from BSN schools. All 6 of the male participants were from pubic schools and 4 of those were CAI-users. One of the male CAI-users was from a medium size school and the 3 others were from large schools. Of the 102 female participants, $80 \%$ of the AD, $57 \%$ of the BSN and $60 \%$ of the diploma school participants were CAI-users. A slightly higher percent, or $72 \%$ of the public school participants were CAI-users as compared to $63 \%$ of the 
private school participants. The analysis by the size of school showed that $80 \%$ of the females from large, $72 \%$ from small and $61 \%$ from medium size schools were CAI-users. So, a summary of the findings of the genders shows a similar pattern as above. For both males and females, those who work for large, public AD schools were more often CAI-users.

The data was analyzed to determine the age-ranges of participants who used CAI and the findings showed that $58 \%$ of the participants in the 20 to 40 -year-old age-group, $65 \%$ in the 41 to 50 -year-old age-group and $74 \%$ of the participants in the 51 and older age-group were CAIusers. In the youngest age-group, 7 of the 12 who used CAI were from AD schools. All 7 of the CAI-users, or $70 \%$ of the public school participants were CAI-users. A total of $75 \%$ of the small school, $40 \%$ of the medium and $67 \%$ of the large school participants were CAI-users. The statistics for the 41 to 50 -year-old age-group showed that 30 out of 46 , or $65 \%$ were CAIusers. A breakdown by the type of school showed that $88 \%$ of all AD participants and only $59 \%$ of BSN participants were CAI-users. A breakdown by the type of school showed that $68 \%$ of all public school and $58 \%$ of the private school participants were CAI-users. The analysis by the school size showed that $62 \%$ of the small, $77 \%$ of the medium and $80 \%$ of the large school participants in the age group were CAI-users. For the 50 participants in the eldest age group from 51 years-of-age and above, $74 \%$ were CAI-users. A breakdown by the type of school showed that $88 \%$ of all $\mathrm{AD}$ and $59 \%$ of all $\mathrm{BSN}$ participants were CAI-users. A breakdown by type of institution showed that $74 \%$ of the public and $73 \%$ of the private school participants were CAI-users. The findings for the sizes of schools showed that $62 \%$ of the small, $77 \%$ of the medium and $80 \%$ of the large school participants in that age-group were CAI-users. 
Integration of CAI 283

To summarize, the age-group that used CAI most was somewhat of a surprise. Instead of the 20 to 40-year-old age-group having the highest percent of CAI-users as would be expected, it was the older age group of 51-years and over that had the highest percent of CAI-users. The other findings were almost identical to the previous findings in this section that showed that participants from large and public and AD schools used CAI most often. The only finding that was different than the other finding was that for the 20 to 40 -year-old age-group, participants from small schools used CAI more.

The next part of the question looked at the educational preparation of the participants and their use of CAI. The findings showed that a total of 7 participants had a BSN degree or lower and of those, 6 , or $86 \%$ were CAI-users. A breakdown by the type of school showed that all 5 $\mathrm{AD}$ and 1 of the $2 \mathrm{BSN}$ participants were CAI-users. All 6 of the participants were from public schools and used CAI. Five of the 6 were from small schools and only 1 was from a large school.

Of the 73 participants with an MSN degree, 63\% were CAI-users. CAI-users included $45 \%$ of the BSN, $75 \%$ of the AD and all of the diploma school participants. A total of $65 \%$ of the public and $56 \%$ of the private school participants with an MSN were CAI-users. A breakdown by the size of school showed that $68 \%$ of the small, $61 \%$ of the medium and $50 \%$ of the large school participants were CAI-users.

There was a total of 28 participants with a $\mathrm{Ph} . \mathrm{D}$. and of those, $79 \%$ were CAI-users. A breakdown by the type of school showed that $75 \%$ of the BSN and $93 \%$ of the AD school participants used CAI. The analysis by the type of institution showed that $81 \%$ of the public and $71 \%$ of the private school participants were CAI-users. A breakdown by the size of school 
showed that $68 \%$ of the large and the one participant from a medium size school used CAI.

The next part of the study investigated some of the "key features" of schools that participants indirectly identified as using the strategies more "frequently" and as being more effective at integrating CAI. The calculations for this part of the study consisted of dividing the sample into two groups, calculating the means for the strategies from Questions 2 through 6 of the ICAI Questionnaire and then comparing to see which group had the most means that were higher.

Key features of effective schools. The first comparison looked at schools that had a computer lab in the SON and those that did not and the findings showed that schools with a lab had a total of 46 means that were higher than schools without a lab. Schools without a lab only had 15 means that were higher. Schools with a lab used the strategies much more "frequently" and perceived the strategies were more effective for all four purposes. The next series of inquiries compared schools that had computers that students used that were located in four different departments of the school. The findings for the first analysis showed that schools that had computers in the "school of nursing" had a 50 means that were higher as compared to 12 means that were higher for schools that did not have computers in the SON. It was not surprising that those schools with computers in the SON not only used more strategies but they also perceived them to be more effective for student learning, decreasing the students' time and deceasing the teachers' time, but not for decreasing costs. The next analysis in the series compared schools with computers in "another department" and the findings showed that schools with computers had 44 means that were higher as compared to 17 means that were higher for others. Schools with computers in "another department" used more strategies and rated them as 
more effective for all four purposes. The third analysis of the series examined schools with computers in the "library" and, for some unexplainable reason, the results showed the exact opposite findings as the other findings in the series. The means for schools that did not have computers in the "library" were 15 and schools that did not have computers in the "library" had 49 means that were higher for frequency of use and all four purposes. It was noted that the means were unanimously higher for the strategies to decrease costs and decrease the teachers' time. The fourth part of the question compared schools where "students purchased" their own computers and the findings were 51 to 11 in favor of schools where they purchased computers in all areas, except costs where each group had 4 means that were higher.

The next inquiry examined the number of computers in three departments to see if the number of computers or the locations of the computers made a difference. The findings for the first comparison showed that schools with "16 or more computers in the SON" had 47 means that were higher as compared to 15 for schools with " 15 or fewer" computers. Schools with the higher number of computers used the strategies more "frequently" and rated them higher in all areas, except decreasing the teachers' time. The findings for the second analysis showed that schools with "31 or more" computers in "another department" had 55 means that were higher and schools with fewer computers only had 23 means that were higher. The findings showed that schools with "31 or more" computers in another department used the strategies more often and perceive them to be more effective for all but "increasing learning" and "decreasing costs." The findings of the third analysis showed that schools with " 25 or more" computers in the "library" had 39 means that were higher as compared with 19 means that were higher for frequency of use and for three purposes, but not for decreasing the teachers' time. 
The next question looked at the number of full and part-time technical and instructional support personnel to see if the human resources that were made available to students and to help teachers made a difference in the frequency of use and participants' perceptions of the effectiveness of the strategies. The first of the three inquiries looked at the technical support personnel and the findings showed that schools that employed " 3 or more" technical support personnel had 53 means that were higher as compared to 8 that were higher for the schools that employed two or less. The findings of the second inquiry found that schools that employed " 2 or more" instructional support personnel had 49 means that were higher as compared to 14 means that were higher for schools that employed one or less. The third study combined the two types of support personnel and the findings showed that, once again, the schools with " 4 or more" technical and instructional support personnel had 52 means that were higher as compared to 10 means that were higher for the schools with "less than 4" support personnel.

Briefly stated, the findings indicated that participants who worked at schools that had more resources, whether they were computers or human support personnel, used the integration strategies much more often and perceived them to be much more effective for all four purposes than those who worked for schools with fewer resources. Also, based on the findings of this small inquiry, the data seemed to indicated that employing technical support personnel had more of an impact than the number of instructional support personnel.

The final analysis in this section examined the percent of teachers from the school that used CAI to see if a small difference in the percent made a difference in the frequency of use or the participants' perceptions of the effectiveness of the strategies. The findings of the first part of the analysis showed that the means for schools where " $41 \%$ or above" of the faculty used CAI 
were actually lower for the ratings of the effectiveness of the strategies than schools where "less than $40 \%$ " of the teachers used CAI. Schools with " $41 \%$ or more" of the teachers used CAI had a total of 27 means that were higher as compared to 35 higher means for schools with " $40 \%$ or less." The second part of the series showed an almost opposite trend in which schools with " $51 \%$ and above" of the faculty use CAI had 37 means that were higher as compared to only 26 means that were higher for schools with " $50 \%$ or less" of the teachers used CAI. A closer examination of the findings showed that while the percent of faculty who used CAI had little bearing on the number of strategies the participants used or their perceptions of the effectiveness of the strategies for decreasing costs or the students' time, there was a positive difference in the way schools where " $50 \%$ or more" of the faculty used CAI perceived the effectiveness of strategies for "increasing student learning"and "decreasing the teachers' time."

The findings of the fourth study question showed that the participants used a total of 5,540 $(\mathrm{M}=50.8)$ software programs during the preceding year. Tutorial software was used most often followed by drill and practice, simulations, virtual reality and expert systems. Associate schools used $78 \%$, or 5 times as many programs as BSN schools that only used $21 \%$ and diploma schools that used $0 \%$ of the total programs. Associate degree schools used more of all 5 types of software than BSN or diploma schools except BSN schools used 5\% virtual reality and AD schools only used 3\%. Associate schools used drill-and-practice programs most followed by tutorials and then simulations. Bachelor schools primarily used tutorials. Public schools used almost 15 times, or $94 \%$ of the software programs as compared to $6 \%$ for the public schools. Both public and private schools used tutorials and drill-and-practice programs most often. Large schools used $66 \%$ of the programs, medium schools used $23 \%$ and small schools only used $11 \%$ 
of the total programs reported. Small schools used more tutorials and simulations, medium size schools almost exclusively used tutorials and large schools used drill-and-practice programs most followed by tutorials.

Of the 2,312 (M=21.2) commercially purchased and in-house produced software programs that were used by the schools, almost all, or 95\% were commercially-purchased. Associate schools used 56\%, BSN schools used 43\% and diploma schools used 1\% of the total programs. Bachelor schools mostly used commercial programs and slightly more of the customized programs than teacher-authored and AD schools used slightly more teacher-authored programs than customized. The diploma schools only used commercially purchased programs. The bulk, or $94 \%$ of the programs were used by public schools and only $6 \%$ were used by private schools. Public schools used mostly commercially-purchased programs with small but almost equal numbers of customized and teacher-authored programs. Private schools almost exclusively used commercially-purchased programs. Medium size schools, in response to this question, reported using more software than either large or small schools. Small schools used mostly commercially-purchased software and a few teacher-authored programs. Medium size schools almost exclusively used commercially-purchased software and large schools used the largest number of customized and teacher-authored programs of the group.

The results of the fifth study question showed that participants indicated their peers were most effective at integrating tutorials, followed by simulations, drill-and-practice, virtual reality and expert systems. When the findings were broken down by the type of school, they showed that participants from $\mathrm{AD}$ schools rated their peers as more effective than BSN schools at integrating all five types of software. Associate school participants rated their peers as most 
effective at integrating tutorials and BSN participants thought their peers did better with simulations. Both AD and BSN schools rated their peers as least effective at integrating expert systems.

A second inquiry showed that public school participants rated their peers as more effective than private school teachers at integrating all five types of software. Both public and private schools rated their peers highest at integrating tutorials and lowest with expert systems. A third analysis that was based on the school sizes showed that participants from large schools thought their peers were the most effective followed by small and then medium size schools. Large school participants thought their peers were most effective with drill-and-practice, small school participants thought their peers integrated tutorials best and medium size school participants thought their peers integrated simulations most effectively.

When asked to rate their peers on effectiveness at integrating commercially-purchased or in-house produced software, the findings showed that not only was commercially-purchased software used much more frequently, but participants also perceived their peers were much more effective at integrating it into their courses. Teacher-authored software was rated second and surprisingly, they rated their peers as least effective at integrating customized software. As before, $\mathrm{AD}$ schools rated their peers as more effective at integrating all three types of software than BSN teachers and both groups thought they integrated commercially-purchased software best and customized least effectively. The findings for the types of institutions showed that public school participants rated their peers as being more effective at integrating all three types of software than private school participants. Both public and private schools rated their peers as most effective at integrating commercially-purchased software and least effective with 
customized software. Large schools rated their peers as most effective at integrating all three types of software than small or medium size schools. Small school participants rated their peers as more effective at integrating commercially-purchased and customized software while participants from medium size schools rated their peers as more effective at integrating commercially-purchased first and then, teacher-authored software.

Pearson $r$ correlations were calculated to determine the strength of the relationships between the frequency of use and effectiveness of the 5 different types of software. The findings for the entire group of participants showed statistically significant relationships $(\mathrm{p}<0.01)$ for virtual reality and expert systems software. The relationships were significant because they were the two types of software that were the least used and least effectively integrated in most instances. A comparison of the correlations by the two types of schools also showed that virtual reality and expert systems software were significantly related for both AD and BSN but the BSN schools also had a significant relationship for drill-and-practice software. The same results, significant relationships for virtual reality and expert systems, were obtained for public and private schools and private schools also had a significant relationship for drill-and-practice software. The correlations for the three different sizes of schools showed that large schools had significant relationships for all five types of software, medium size schools had a statistically significant relationship for virtual reality software only and for small schools, there were significant relationships for virtual reality, expert systems and drill-and-practice software.

Correlations were also calculated for the commercially-purchased and in-house produced software for the entire group and the findings showed that there was a significant relationship for customized and teacher-authored software $(\mathrm{p}<0.01)$. A correlation to compare the two types of 
schools showed that BSN schools had significant relationships for customized and teacherauthored software and AD schools for teacher-authored software only. The third correlation based on type of institution showed that there was a significant relationship for public schools for customized and teacher-authored software and for private schools for commerciallypurchased and teacher-authored. The correlation based on school sizes showed that large schools had significant relationships for customized and teacher-authored, medium size schools

for teacher-authored only and small schools for commercially produced, customized and teacherauthored.

CAI-users schools. There were three different answers to the question of how many and what types of schools used CAI in this study and, while the answers to the questions differed based on the numbers and mixes of the respondents, the findings were close and provide a range of figures that will help to clarify and update what is currently known and available in the literature.

In response to Question 1 of the questionnaire, the findings showed that $71 \%$ of the participant's schools used CAI and $29 \%$ did not. The percent rose to $77 \%$ when a different number and mix of participants responded to Part A of Question 18. Then, in response to part B of the same question, the findings showed that $26 \%$ of the respondents either did not respond or indicated that none of the faculty at their school used CAI, which meant that $74 \%$ were CAIusers. Therefore, the answer to the question of how many nursing schools in the country use CAI was somewhere between $71 \%$ and $77 \%$. This finding is a smaller range and much more up-todate figure than most recent $55 \%$ to $77 \%$ range that was published well over a decade ago by Milkan and Aiken (1992). Since the previously published findings were so out-of-date, they 
were totally unreliable in light of the number of changes that have taken place in the areas of computer technology within nursing and higher education over that time period. It is also important to note that all three of the findings of this study are well below the reports of "almost universal use" by all nursing schools in the U.S. that were reported in 1998 by Carthy and Rosenfield.

The findings to both the first and the eighteenth question showed that a higher percent of all associate and diploma schools used CAI than BSN schools and that a higher percent of public schools used CAI than private schools. The responses to both questions also showed that a larger percent of large schools used CAI as compared to medium or small schools. Of particular interest were the findings of the first question that showed that while medium size BSN schools used CAI more than small or large BSN schools, a much higher percent of the small AD schools used CAI than the medium and that a very small percent of large AD schools used it. Small diploma schools used CAI more than medium or large diplomas according to the first question.

The answers to part "B" of Question 18 showed that 28 respondents indicated " $0 \%$ " of the faculty at their schools used CAI. Also surprising was the finding that 78, out of 109 respondents indicated that " $50 \%$ or less" of the faculty at their schools used CAI. At the other end of the spectrum, $25 \%$ of the schools indicated that between "80 to $100 \%$ " used CAI. The first frequency distribution chart showed that $72 \%$ of the schools indicated that less than $50 \%$ of the teachers at their schools used CAI but also that there were a substantial number of schools that have close to "universal" use.

When the two types of schools were compared, the findings showed that 17 responders from BSN schools and 10 from AD schools said "0\%" of the faculty at their schools used CAI. 
One of the most startling findings of the study was the frequency distribution chart that showed that 27 participants from AD school indicated that $50 \%$ or more of the teachers at their schools used CAI as compared to 3 participants from BSN schools. The breakdown by the type of institution showed that public schools had a higher number of participants who indicated " $0 \%$ " of the faculty at their schools used CAI but they also had a much higher number that indicated $51 \%$ or higher of the faculty at their schools used CAI. The breakdown of the data by size of schools showed that the small schools had 17 participants who indicated $51 \%$ or above of the faculty at their schools used CAI as compared to 6 from medium and large schools.

To summarize, the findings of this study differed from reports in the literature that indicated that BSN schools used CAI more often than AD schools. The finding that the percent of public schools that used CAI was only slightly higher than private schools was surprising given the vast differences in the resources that were evident elsewhere in this study. The fact that a higher percent of large than medium or small schools were CAI-users is new to this study and may contradict Cambre and Castner's (1993) findings that many large, well-equipped institutions were low CAI users.

Some of the most impressive findings of the study came from the frequency distribution tables (See Tables C 57 through C 60) that showed such a large number of schools reported " $0 \%$ " of the faculty used CAI. On the other hand, the synergistic effect of a few faculty inspiring others described by Cambre and Castner (1993) was also evident with the fairly large number of schools that indicted $51 \%$ or above of their faculty used CAI.

Of particular importance was the finding that $17 \mathrm{BSN}$ participants and only 10 from AD schools reported " $0 \%$ " of the faculty used CAI and conversely, that 27 AD and only 5 BSN 
participants indicated $51 \%$ and above of the faculty at their schools used CAI. This finding not only supports the findings of from Question 1 and Part A of the same question, but it shows that the non-users are not just individuals but entire schools and that most of the non-users in the area of nursing education are BSN schools. The fact that participants from 26 public schools indicated $51 \%$ and above of the faculty at their schools used CAI was not a surprise given the resources they have at their disposal, but the fact that 17 participants from small schools as compared with only 6 from medium and 6 from large schools reported $51 \%$ and above of the faculty used CAI does suggest that resources are not as strong a determining factor as was assumed and supports the findings of the Cambre and Castner (1993) study. Participants

The findings concerning the characteristics of the participants precisely matched the profile that is so often put forth in the literature as a description of nursing teachers throughout the United States. The gender profile of $94 \%$ females to $6 \%$ males, the predominance of masters educational preparation, and the fact that a large majority were over the age of forty-years-of-age were all characteristics that perfectly match the way the profession has been described in all types of published literature over the past twenty years (Adams et al., 2002).

Frequency of Use of the Strategies

There were several important findings that seemed almost instantly evident concerning the strategies that were "frequently" used. The results showed that 29 of the 44 strategies, or $66 \%$ were "frequently" used. The strategies included 11 of the 19 strategies to increase student learning, 4 of the 8 to decrease course costs, 5 of the 7 to decrease the students' time and 4 of the 10 to decrease the teachers' time. It was interesting to note that a higher percent of the strategies 
that were "frequently" used for strategies to decrease the students' time (71\%) followed by increase student learning (58\%), followed by strategies to decrease costs (50\%) and finally, by strategies to decrease the teachers' time (40\%).

First, participants indicated that a large majority used the four preinstruction strategies of "explain the CAI, explain the objectives, motivate and integrate" "frequently." The findings were supported by numerous recommendations in the literature concerning the strategies. The first stressed the importance of "explaining the CAI" in order to prepare the learners by telling them what to expect. The purpose is to decrease the time it takes to begin learning and prevent the learner from being distracted by concerns about how to operate the computer or navigate the program (Khoiny, 1995; Perciful \& Nester, 1996). The second strategy of "explaining the objectives" is also a basic tenant of most instructional design theories and several researchers recommended the teacher clearly state the goals and objectives of instruction beforehand even when they appear within the material (Gagne', 1985; Gustafson \& Powell, 1991). The third strategy of "motivating" learners by explaining the value of the content helps learners identify why it is important, how it relates to what is already known, or the rest of the course, and how it may be of value in the future (Gustafson \& Powell, 1991). The fourth strategy of "integrating" the CAI with the rest of the course helps to simulate the recall of prerequisite knowledge which is especially important when students are learning complex, integrative skills, such as clinical decision-making where they need to have relevant prerequisites in mind so they can integrate them with new ideas (Gagne', 1985).

The finding that the "pretest" strategy was used so much less "frequently" than the other preinstruction strategies seemed reasonable since the literature only recommends pretesting a 
new CAI program once before purchasing or using it while the other strategies would, and should, be used each and every time a CAI program is assigned. However, the item-by-item analysis showed that $72 \%$ indicated they "never" pretest software and only $28 \%$ said they ever pretested. The importance of pretesting each software program was stressed by experts from many different fields who noted, among other things, that the programs needed to closely match the course objectives, the skills, and content of the course, as well as the needs and abilities of the students (Cobb, 1999; Dick \& Carey, 1991; Gleydura, Michelman \& Wilson, 1995; Goyne, McDonough, \& Padgett, 2000; Griepp, 1988; Kemp, Morrison and Ross, 2001; Khoiny, 1995). The person evaluating the software also needs to ensure students have adequate computer skills to use it before it is assigned (Cobb, 1999). The consequences of not pretesting are fairly serious since the use of poorly designed or the inappropriate application of software not only detracts from the course objectives but it wastes the students' time and may turn them against the media in the future (Gleydura et al., 1995).

Another related finding showed that $42 \%$ of the participants indicated they "never" "evaluated CAI." While the finding seems realistic and honest, it was also contrary to good teaching practices. More specifically, Dick and Carey (1991) recommend evaluations be conducted during the post-instruction period to ensure students who use the CAI program accomplish the course objectives and for the purpose of collecting student feedback about the CAI learning experience so that appropriate adjustments can be made to the course in a timely fashion. The primary rationale for conducting a summative evaluation of student learning is to provide decision-makers with information about revisions that need to be made. Such revisions should be anticipated in every course and can range from minor to major changes but they 
cannot be made if an evaluation does not take place (Gustafson \& Powell, 1991). The fact that $45 \%$ of the participants conducted a summative evaluation of the software "each semester" was more encouraging than the findings for the "pretest" strategy but both findings suggest the strategies are not being used by often enough or by enough participants for the CAI to be "effectively" integrated into the course.

The findings showed that there was a large difference between how often the different modes of instruction were used. More specifically, 92\% indicated they used an "individualized" mode of instruction at least once a semester while just over half, or 52\% used a "small-group" mode and only $39 \%$ used CAI for "whole class" teaching once a semester. The fact that CAI and self-paced learning have become almost synonymous terms over the years may help to explain why it is the preferred mode. It is also possible that the finding may simply be a reflection of the many variables the teacher needs to consider when deciding on which mode to use such as the course objectives, the availability of computer hardware and software, classroom facilities, students' computer skills, the number of students in the class, and the teachers' level of comfort and expertise with computers that were noted by Khoiny (1995). The fact that only a few nursing teachers have the technical skills to use CAI for a whole-class presentation was not explicitly stated, but could easily be inferred from the findings of both the Austin (1999) and the Lewis and Watson (1997) study.

The literature discusses the advantages of an "individual" mode of instruction more than the other modes of instruction. The primary benefit that is most often discussed is that selfpacing allows the learner to tailor the lesson to their knowledge level, learning style (Smith, Jones \& Waugh, 1986), academic background, (Conklin, 1983), interests and rate of learning - 
(Levin, 1983). An individual mode has been shown to promote motivation and the development of critical thinking, problem-solving and improved memory (Charp, 2001) and it is more attractive for adult learners who appreciate the convenience of learning any place, any time (Knowles, 1984; Reif, Roulis, \& Huber, 1995; Scanlan, 1986). Some of the newer developments in software design that combine artificial intelligence and multimedia displays that dynamically adjust the instructional strategies based on how the student responds to each unit of instruction (Gibbons, Fairweather \& O’Neal, 1993) are clearly designed with an “individual” user in mind but will also help to reinforce the notion that CAI is a way to provide each student with a "private tutor."

One of the major reasons a "small-group" mode of instruction is used instead of "individualized" is to provide students with practice in collaboration and negotiation skills for the purpose of preparing students for team-work. Others are to save time, accommodate shortages in computers or software programs and provide academically weaker students with peer support (Cobb, 1999; Park, 1993). Several researchers noted the greatest benefit of a "small-group" mode is that the social interaction and the active exchange of ideas facilitates problem-solving and learning (Bejarano, 1987; Wizer, 1987). Since nurse educators use smallgroup work as a strategy in other venues, it may have simply escaped their attention that they could also use it with CAI.

One advantage of a whole-class mode of CAI instruction is that it permits the teacher to control the pace of the instruction, thereby, ensuring the entire class has mastered the material before they progress to the next lesson (Straker, 1988). However, the primary benefit is the same 
as with small-group work, which is that knowledge emerges through the student-to-student interaction rather than through the delivery of subject content by the teacher (Goodman \& Blake, 1996).

Technical and instructional support personnel were the two most frequently used strategies of the 19. Thede et al. (1994) noted the importance of technical support personnel who needed to be available to ensure students were able to access the learning material, to provide assistance to students who need help using the computers or software and to solve technical problems. There was almost nothing on the topic in the literature, however, the findings of this study showed that the participants used "instructional" support personnel and thought they were as "effective" for improving learning outcomes and for saving the teachers' and the students' time as technical support personnel.

Another important finding that was different from the reports in the literature was that participants indicated they assigned CAI as a "required" part of the course $(\mathrm{M}=2.99)$ more often than they made it an "optional" assignment $(\mathrm{M}=2.35)$. The individual item analysis showed that $51 \%$ of participants indicated they designated CAI as a "required" assignment and only $38 \%$ made it as an "optional"assignment each semester. While the two strategies are certainly not mutually exclusive and the same teacher who assigns one software program as "optional" may, and probably does, assign another program as "required," the fact that they used the "required" strategy more often matches the recommendations of the experts in the field. The primary reason given for not assigning CAI as an "optional" assignment was that it has learning consequences. The results of several studies showed that busy students seldom do "optional" assignments (Bates, 1999) and that they often prejudge "optional" material as being out-dated 
and do not treat it as a priority (Thede et al., 1994). The other reasons are that there are serious cost and time consequences associated with assigning CAI as an "optional" assignment. The cost implications will be discussed later, but when the teacher fails to include the information from an "optional" assignment on the course test, which is more often the case than not, students who have done the assignment quickly become discouraged and count the assignment as a waste of valuable study time (Thede et al., 1994). As a result, they quickly learn to stop doing the "optional" assignments and spend their time doing assignments that count toward the course grade. In terms of the teachers' time, there are few teachers who would not teach important information that is included in an "optional" assignment using some more traditional method. The result is that both the teacher's and the students' time, at least those who did the optional assignment, is nearly doubled for the unit of instruction.

Another important finding was that participants indicated they seldom used CAI to "replace traditional" instruction. The findings of this study showed that only $35 \%$ used CAI to "replace traditional" teaching each semester but that $41 \%$ never did. Several dated nursing studies and articles reported the similar findings (Carty \& Rosenfeld, 1998; Mikan \& Aiken, 1992; Oermann, 1997). The reason this finding is important is that they not only confirm those reports, but it a record of the thoughts, practices, and values of a group of 77 professed CAIusers. The cost and learning implications of this finding are numerous because, as mentioned so many times before, when CAI is used as a supplement to the traditional teaching method, the productivity of the course falls not only because the unit costs of instruction are duplicated but because both the teachers' and the students' time are nearly doubled (Bates, 1999; Dean, 1989). As noted previously, CAI is simply too costly to use as a supplement to another costly delivery 
system such as a teacher-directed lecture or a clinical discussion group.

Of the three post-instruction strategies, the use of "follow-up" activities or conducting a "post-instruction" discussion or critique were both used "frequently" while the other strategy of providing "alternative" means of restudying the material was not. The purposes of the "postinstruction" and "follow-up" strategies are to help students integrate the CAI content with the rest of the course and to enhance retention and the transfer of knowledge (Anderson, 1990). Kemp et al. (2001) noted teachers may, or may not, decide to assign an "alternative" remedial assignment for students who fail to accomplish the course objectives using the CAI program. It depends on the number of students who failed and the level of maturity of the learners in the class. Based on the mean for the strategy it is seldom used in nursing education.

Including CAI content as part of an "in-class exam" was "frequently" used but participants indicated they seldom used "embedded" test grades as part of the course grade. There may be several reasons teachers seldom use the "embedded" test grades but the most probable is that the commercial programs that are currently on the market are not constructed in such a way that allows the teachers to securely save and download the grades from "embedded" tests. Another might be that the teachers prefer to have students use the "embedded" tests for practice to prepare for an in-class test where there is more security.

According to the literature, either testing strategy has the potential to increase learning but it is important that they use one or the other since they both promote the perception that the CAI assignment is an integral and important part of the course which makes students feel the time spent on the assignment is worthwhile ( Kemp et al., 2001; Thede et al., 1994). The use of "embedded" test questions certainly has the potential to save more of both the teachers' and the 
students' time than including the content on an "in-class" examination which is a finding that should be brought to the attention of not only the teachers, but also the commercial software developers.

\section{Effectiveness of the Strategies for Learning}

As noted earlier, the means and patterns of responses indicated that participants used the strategies they perceived to be most "effective"for increasing student learning. The exact same strategies that were above 2.50 for the "frequency" of use also had a mean that was above 3.00 for the perceived "effectiveness" of the strategies for increasing learning.

Four of the 5 preinstruction strategies were perceived to be "effective" for increasing student learning. That participants perceived the 4 conceptually similar preinstruction strategies, "explain the CAI, explain the objectives, motivate and integrate" were "effective" for increasing student learning corresponds with numerous recommendations in the literature (Khoiny, 1995; Perciful \& Nester, 1996).

The fact that they seldom used the "pretest" strategy was somewhat surprising since they used the other strategies to prepare the learner so often. However, the fact that they did not perceive it to be "effective" for increasing learning was even more of a surprise. It is also the opposite of how almost every instructional design book describes good teaching practice. It is difficult to imagine why so many educators would think "pretesting" the software was unnecessary unless it is because they make so many other decisions, such as planning the curriculum, selecting teaching strategies, and deciding which textbook students will use that they believe they know the content of the courses and students well enough to make the decision without any up-front feedback. 
Three of the 5 mid-instruction strategies were "effective" for increasing student learning. They included providing "technical" and "instructional" support and the "individual" mode of instruction. The findings closely resemble the Thede et al., (1994) findings that showed that faculty thought ensuring there are technical and instructional support personnel available to assist students who need it, either by phone or on-site, positively impacted both learning and time. Cambre and Castner (1993) also noted that technology-using teachers were as concerned about the technical problems that were bound to happen and how disruptive the lost time would be to the course as they were about the costs of the technology.

While the "individual" mode of instruction was perceived to be "effective" for increasing student learning, the use of a "small group" and a "whole class" mode of instruction were not. The findings may be due to the fact that participants have not considered using a "small-group" mode of instruction with computers or that they do not think the benefits outweigh the many drawbacks of small-group work (Bejarano, 1987; Wizer, 1987). The fact that a "whole class" mode of instruction can be effective was evident in an account by Goodman and Blake (1996) in which they describe the dramatic impact a multimedia program had when they used it to create a participatory interactive learning environment in which nursing students were actively involved in decision-making as a group. The power of the exercise only became apparent to the teachers when the entire class visibly grieved when the "virtual" patient they were making decisions for died. 
Four of the 9 post-instruction strategies that were "effective" for increasing student learning included designating CAI as a "required" assignment, including CAI content in a "course exam," and the use of "follow-up" activities and "post-instruction" discussions or written critiques.

The first, assigning CAI as a "required" part of the course rather than as an "optional" assignment has been shown to positively impact student learning as in the studies by Collis and Moonen (2001) and Greipp (1988) where students simply did not complete or even attempt to do “optional” technology-delivered assignments.

The next two strategies that were rated as "effective" included conducting a "postinstruction" discussion or written critique and the use of "follow-up" activities. The purpose of the strategies is to reinforce what the students learned from the CAI assignment, correct any misunderstandings (Kemp et al., 2001) and provide the teacher with feedback about the correctness or incorrectness of the student's performance. Learning improves when students are given both intrinsic (from the computer) and extrinsic (from the teacher) feedback that explains the level of accuracy, why some answers are correct and others are not and, according to Laurillard (1993), the sooner the feedback is provided the better and the more precise and individualized it is, the better the learning outcomes. The strategy of providing follow-up activities so that students use what they learn in the CAI program not only enhances retention, but it also helps in the transfer of knowledge (Anderson, 1990).

The next "effective" post-instruction strategy for increasing student learning was including CAI content as part of the "course exam." While the literature does describe the effectiveness of the strategy for increasing student learning, of the two testing strategies, it is less 
effective since the time between learning and feedback is much longer and it doubles both the teachers' and the students' time. The other testing strategy of including the student's grades that were earned on "embedded" tests was not perceived to be "effective." One of the ways the use of "embedded" CAI assessment scores increases learning is that it takes advantage of the unique benefit of interactive CAI testing that asks the student perform the task (i.e., make a clinical decision) rather than simply describe how it is done (Gibbons \& Fairweather, 1998).

The use of CAI to "replace traditional" instruction was not perceived to be "effective" for increasing student learning. As mentioned previously, the finding was reported by several older studies in the literature that found that most nurse educators integrated CAI as a supplement to the traditional teaching method in both the classroom and clinical area (Mikan \& Aiken, 1992) despite considerable evidence that when used as a supplement, CAI seldom improves learning enough to justify its use over many other less expensive teaching methods (Hmelo, 1990).

The last post-instruction strategy that was not thought to be "effective" for increasing student learning was providing students who fail to meet the course objectives using CAI with "alternative" methods for re-studying the content. As previously discussed, the decision about whether to use the strategy depends on a number of factors (Kemp et al., 2001). However, in light of the finding that only $45 \%$ of the participants do a summative evaluation of CAI learning and that most teachers include questions about the CAI content on a course exam and therefore, probably do not evaluate student learning from the CAI separately, it seems that the combination of practices might make it difficult to ascertain which students accomplished the learning objectives using CAI and which ones did not. It is possible that the two findings are closely related and that a failure to adequately evaluate learning results in the failure, on the part of the 
Integration of CAI 306

teachers, to recognize the need to do something even as simple as making a recommendation that the students review the material in the textbook.

Effectiveness of Strategies for Decreasing Costs

The findings showed that for the 8 strategies to reduce costs, 4 strategies were "frequently" used but none were perceived to be "effective" based on the 3.00 mean criteria. Even though none of the strategies were perceived to be "effective"for decreasing costs, the literature has much to say about the strategies and how teachers might use them to decrease course costs.

According to the literature, one of the strongest strategies for decreasing costs is using CAI to "replace traditional" instruction. This is uniquely true of nursing education simply because it is a clinically-based profession. Because it is, nursing schools must hire large numbers of instructors to teach the clinical courses and so, the costs of providing a nursing student with an education are much higher than other schools within higher education. Therefore, anytime even an expensive instructional delivery system such as CAI can be used to replace some portion of class room or clinical time, a substantial cost savings can be realized (Perciful \& Nester, 1996).

There are many examples in the literature of nurse educators using CAI to replace the traditional method of teaching and at the same time, substantially reducing the educational costs (Engberg \& White, 1998). One of the best examples was when Bareford (2001) used a popular, inexpensive off-the-shelf game called Sim City to replace the traditional method of teaching conceptually complex principles of community health nursing to graduate level students.

Bates (1999) looked at it from a different perspective when he noted that cost- 
effectiveness with CAI could be only be achieved by using each program "optimally." Since most CAI programs are so complex and information-dense, they can be used many times by the same learners without significant repetition either in the same year or over consecutive years to teach different skills or content (Bates, 1999). Another suggestion to "optimize" the use of CAI was for teachers to reorganize their courses so that the teaching that can best be done by CAI moves away from fixed, scheduled group instruction to individualized or small-group modes of learning (Bates, 1999). The goal is a balance between face-to-face instruction and technologybased teaching that is based on the needs of each class (Bates, 1999; Dean, 1989).

The finding that participants did not perceive providing "technical" or "instructional support" decreased costs was supported by the literature. Several educational economists noted that the ongoing costs for support personnel (technical and instructional) salaries were so expensive that when they are included in the budget, they significantly increase the course costs (Dean, 1989; Levin, 1983). Massy and Wilger (1998) noted that because the costs of purchasing and then providing support for CAI were so much higher than for other instructional delivery media, cost-effectiveness can be achieved, but only through the learning gains and time-savings benefits they provide and not because it reduces the costs of the course. The findings of this study did contradict a comment by Bates (1999) in which he noted that few educators were aware of the implications of course budgets or the costs of providing technical or instructional support services because those types of concerns typically fell under the responsibility of school administrators. The participants obviously recognized the implications of providing support personnel as indicated by their responses.

None of the three modes of instruction were perceived to be "effective" for reducing 
costs. However, the literature mentions several cost implications associated with the different modes of instruction. For instance, Khoiny (1995) suggested a way teachers could solve the problem of insufficient computer resources (hard and software) was by dividing a large group of students into small groups, rather than assign them to work "individually" and then have them complete the assignment at scheduled times. Bates (1999) noted the costs of an overhead projector system or monitor that was large enough to permit viewing a CAI program by an entire classroom of students might cost so much that the technology may be too expensive for some schools.

\section{Effectiveness of Strategies for Decreasing Students' Time}

The findings showed that there were 5 strategies that were "frequently" used but only 3 of the 7 strategies for decreasing the students' time were perceived to be "effective" for that purpose. More specifically, providing "technical" and "instructional support" and the use of “explain CAI" had means that were above 3.00.

The means for the two strategies of "explain CAI" and "explain the objectives" were close (3.19 and 2.99) and in other parts of the study both were perceived to be "effective." Therefore, this finding was insignificant and will not be explored here.

The evidence in this study was overwhelming in that it was noted that $95 \%$ of the participants indicated they perceived "instructional" and "technical" support were effective strategies for saving the students' time. This finding is somewhat different than the literature that said the need for instructional support personnel depends, to some extent, on how diverse the students in the course are, the level of difficulty and complexity of the course content and the objectives of the course (Bates, 1995a). 
While none of the "modes of instruction" were perceived to be "effective" for reducing the students' time, the percentages for the individual item responses showed that $73 \%$ of the participants "agreed"or "strongly agreed" that the "individualized" mode was "effective"for saving the students' time but participants were divided in half about whether or not "smallgroup" or a "whole class" mode did. According to some older research findings, an "individualized" mode of instruction was shown to reduce learning time by as much as 50\% (Larson, 1982; Tilson, 1986) and could reduce by one-third (Bowell, 1995) to two-thirds the time it takes for an entire course (Bitzer \& Bordreaux, 1969). The time difference was attributed to improved organization and design of the instruction or possibly to the individualized interaction and feedback (Bates, 1995a). One of the problems with the "small-group" mode of instruction was that some students completed the assignment more quickly than others, which made it difficult to schedule a post-instruction group discussion (Perciful \& Nester, 1996). Noell and Carnie (1989) noted that the "group mode" of instruction was more time-efficient than the "individualized" mode but did not result in a loss of achievement (Noell \& Carnie, 1989). Again, the major advantage reported in the study by Bareford (2001) who used the Sim City game to teach community health principles was the amount of time it saved the students who went from spending weeks to complete a community-wide assessment project to learning the same principles in a few hours.

\section{Effectiveness of Strategies to Decrease the Teachers' Time}

Among the strategies for decreasing the teachers' time, only 3 of the 10 were perceived to be "effective" for that purpose. The "effective"strategies included "technical" and "instructional" support and an "individual" mode of instruction. 
Integration of CAI 310

The analysis of the percentages of the individual item responses showed that $75 \%$ of the participants "agreed" or "strongly agreed" that the use of an "individual" mode of instruction was "effective" but only 59\% thought "small-group" and $48 \%$ thought a "whole class" mode was "effective"for decreasing the teachers' time. Bates (1999), on the other hand, saw no difference in individualized and a small-group mode when he noted that a major advantage of CAI was that teachers could save time by using CAI to replace the usual classroom or clinical instruction by assigning it as a self-paced learning activity for students working alone or in small groups outside the classroom. Another teacher time-saving strategy proposed in the literature was to use CAI software for a whole-class presentation as a part, or to entirely replace the conventional mode of instruction (Goodman \& Blake, 1996). Thede (1999) noted that simulations, virtual reality and tutorial programs were being used in the classroom to visually illustrate difficult principles and concepts and to reduce the amount of time it normally takes for a lecturediscussion class.

Neither the "course exam" nor the "embedded" test strategies were perceived by participants to be "effective" for decreasing the teachers' time. Both findings seem to run counter to what one would expect to find. Even more curious is that of the two, $56 \%$ either "agreed" or "strongly agreed" that the "course exam" strategy was "effective" while only $47 \%$ perceived using "embedded" tests saved the teachers' time. The findings strongly support the idea that the software that is being purchased it not designed by commercial producers in a way that allows teachers to save time using "embedded tests" that are equipped with automated methods of evaluating and reporting students' grades (White, 1995). However, the findings do suggest that many teachers were able to save time re-using test questions from embedded CAI 
tests as part of an in-class test to eliminate, or at least reduce, the time the teacher spends writing test questions while still integrating the content of the CAI into the course (White, 1995).

Saving the teachers' time for test preparation was only one among a long list of advantages of computerized testing that is "embedded" in the software. Some of the others were that "embedded" tests promote self-directed learning, they allow the student to select the testing time, provide immediate feedback, provide greater standardization of the testing environment and reduce both the teachers' and students' time at the end of the test by eliminating the need to recapture students' attention following an in-class exam (Bloom \& Trice, 1997). The finding that $54 \%$ either "disagreed" or "strongly disagreed" that the use of the scores from CAI "embedded" tests was an "effective"strategy for saving the teachers' time was surprising. As mentioned previously, this information needs to be made available to teachers and software developers so that the more "effective"strategy can replace the less "effective"one.

It was interesting to note that even though the strategy was not considered to be "effective" for any of the three purposes, $73 \%$ of the participants did perceive using CAI to "replace traditional" instruction was "effective" for saving the teachers" time. A comparison of the means showed that the strategy was rated highest for saving the teachers' time $(M=2.82)$ followed by increasing student learning $(\mathrm{M}=2.77)$, which was followed by decreasing costs $(\mathrm{M}=$ 2.61). The idea that using CAI to "replace traditional" instruction would decrease the teachers' time and reduce costs follows closely with the general theme of the literature that those were the two major benefits to be derived from using the CAI. However, the fact that the participants thought it was more effective for increasing student learning than for reducing costs should be explored further. 
The findings of this study were similar to what the literature states so clearly concerning the use of the "optional" or "required" strategies for saving the teachers" time. The findings of this study showed that $46 \%$ of the participants thought designating CAI as an "optional" assignment was "effective"but $65 \%$ agreed that making it a "requirement" saved the teachers' time. Based on the means, participants not only used the "required" strategy $(M=2.99)$ more often than the "optional" $(\mathrm{M}=2.43)$ strategy but they perceived it was the only one of the two that was "effective" for increasing student learning. While it was not perceived to be "effective" the means were higher for decreasing costs and decreasing the teachers' time than for the "optional" strategy.

The fact that 15 of the 9 strategies for increasing student learning were statistically significant correlations and there were so few significant correlations for decreasing costs, students' time and the teachers' time suggests the participants were able to relate the use CAI to student learning more readily than they were to other three purposes. To further illustrate the degree of the disparity, $79 \%$ of the strategies to increase student learning were significant correlations while only $25 \%$ of the strategies to decrease costs, $29 \%$ to decrease the students' time and $10 \%$ to decrease the teachers' time.

The 7 strategies that had relatively weak statistically significant correlations but that were "frequently" used and "effective" for increasing student learning included the four preinstruction strategies of "explain CAI, explain objectives, motivate, and integrate," the mid-instruction strategy of "individual mode," and the two post-instruction strategies of "course exam" and "follow-up." The strategies of provide "technical" and "instructional support"consistently had the highest means for both frequency of use and effectiveness for every purpose except 
decreasing costs but, as discussed previously, the correlations for the two strategies were extremely weak across the board.

The findings showed that only 2 of the 8 strategies for decreasing costs, and 2 of the 7 for decreasing the students' time and 1 of the 10 for decreasing the teachers' time were statistically significant correlations. None also met the other two criteria of being used "frequently" and “effective." In summary, the fact that the strongest correlations were for strategies that were seldom used and not perceived to be "effective" indicated there was stronger solidarity among participants about what was not "effective" and therefore, not used than there was about what was "effective" and therefore, used "frequently." Said differently, the correlations showed that participants agreed less about what worked than they did about what didn't work and that their major focus when using CAI was on strategies to increase student learning. The correlations also seemed to indicate there was little agreement about which strategies might decrease costs or student's time or their own time.

\section{Key Features of Effective Schools and Educators}

The purpose of the next section was to determine if there were key features that could be identified that might distinguish schools that integrated CAI "effectively" from schools that did not. As noted previously, a comparison of the means of the frequency of use of the strategies by the three types of schools revealed some interesting findings. The findings showed that of the 44 strategies, BSN schools used 36 "frequently," while the AD schools used 28 and diploma schools only used 22 of the strategies "frequently." The conversion to percentages showed that BSN schools used $81 \%$, AD schools used $63 \%$ and the diploma schools only used $50 \%$ of the strategies "frequently." There was less of a difference between the schools for the strategies they 
used "frequently" to increase student learning than for any of the other three purposes. More specifically, the percentages showed that the differences between BSN and AD schools were highest for the strategies to decrease the students' time (29\%), followed by the strategies to decrease costs $(25 \%)$, which were followed by the strategies to decrease the teacher' time (20\%) and the smallest difference was for the strategies to increase student learning (10\%). Some of the strategies that BSN schools used "frequently" for the four different purposes that AD and diploma schools did not use included "small group," "whole class," and using CAI to "replace traditional" instruction. All three were recommended as "effective" strategies for integrating CAI into the course in the literature. On the other hand, AD and diploma schools used the “optional" strategy "frequently" for all four purposes which was not recommended in the literature as an "effective" strategy for integrating CAI for any of the four purposes.

The findings for the public and private schools showed that public schools used 29 and private schools only used 23 strategies "frequently." The comparison of the three sizes of schools showed that medium and large schools used almost the same number of strategies "frequently" while small schools used about one half as many as the other two "frequently." More specifically, medium schools used 34 and large schools used 32 strategies, while small schools only used 19 strategies "frequently."

\section{Comparisons of Effectiveness of Strategies for Learning}

When the three types of schools were compared for their perceptions of "effectiveness" of the strategies to increase student learning, the findings showed that BSN schools thought 13 of the 19, AD schools rated 12 and diploma schools did not rate any strategies as "effective." When the findings were combined, BSN schools had 12 strategies that were both "frequently" 
used and perceived to be "effective"and AD schools had 11 strategies that met both criteria. The strategy that BSN schools alone used "frequently" and rated as "effective" was "replace traditional" instruction.

A comparison of the two types of institutions revealed public schools rated almost all of the strategies as more "effective" than private schools. Public schools thought 12 strategies were "effective" and private schools rated 9 strategies as "effective." When the findings for "frequency" of use and "effectiveness" were combined, public schools had 11 strategies and private schools indicated 8 strategies that met the criteria for both. For the most part, the two agreed on the pre and mid-instruction strategies, but only public schools thought "postinstruction, follow-up," and "course-exam" were "effective"strategies for increasing student learning.

The analysis of the three sizes of schools showed that medium and large schools rated 13, while small schools only rated 8 strategies as "effective." Medium and large size schools both had 12 strategies that were both "effective"and "frequently" used while small schools only had 7 strategies that met both criteria. As with the private schools, small schools were far behind the others in their ratings of the "effectiveness" of the post-instruction strategies.

In summary, there were not any outstanding differences between BSN and AD schools for their ratings of the "effectiveness" of the strategies for increasing student learning.. The sample size for the diploma schools was too small to reach any insightful conclusions about that group. While public schools used almost all of the strategies slightly more "frequently" and perceived them to be slightly more "effective"than private schools, the same conclusion could be drawn there. Neither of the institutions thought a larger number of the strategies were 
Integration of CAI 316

"effective" than the other. However, the findings for the different sizes of schools indicated that small schools used many of the strategies less "frequently" and they perceived far fewer of the strategies were "effective" than either the medium or the large schools. Also, with minor variations, large and medium size schools were so similar in their "frequency" of use and their ratings of "effectiveness" of the strategies.

\section{Comparison of Effectiveness of Strategies to Decrease Costs}

The comparison of the means of the three types of schools for the strategies to decrease costs showed that none thought any of the strategies were "effective" for that purpose. While the diploma schools rated the strategies much lower than the other two, the differences between the BSN and AD schools were minor. The same findings were noted for the comparison of the public and private schools as for the previous findings. Neither type of institution thought the strategies were "effective" but the means for the public schools were slightly higher for all eight strategies than the private schools. Once again, small schools perceived the strategies were less "effective"for decreasing costs than medium schools, and medium size schools perceived the strategies were a little less "effective"than large schools.

There were several interesting findings that were noted from this question. The first was that private schools rated the "technical" and "instructional" support strategies as two of the least "effective"strategies for decreasing costs while public schools rated the strategies near the top. Since the literature supported the idea that the costs associated with technical and instructional support personnel were two of the often expensive, on-going costs of CAI, the findings suggest that private school participants may simply be more aware of the costs of providing support services than public school employees. Also, while the mean was not high enough for it to be 
"effective," the analysis of the item-by-item responses showed that a majority did perceive using CAI to "replace traditional" instruction was an "effective"strategy for decreasing costs. A third was that while a majority thought designating CAI as a "required" assignment was not “effective," an even greater number thought making it an "optional" assignment was even less "effective" for reducing costs.

\section{Comparison of Effectiveness to Decrease Students' Time}

The analysis of the effectiveness of the strategies for decreasing the students' time showed that both BSN and AD schools rated 4 of the 7 strategies as "effective" while the diploma schools did not rate any strategies as "effective." While public schools rated 4 and private schools rated 3 strategies as "effective," but the differences between all 7 of the means were minor. A comparison of the three sizes of schools showed that large and medium size schools both rated 5 while small schools only rated 3 strategies as "effective."

One finding of interest was that all three types of schools rated provide "technical" and "instructional" support as the two highest ranked strategies for saving the students' time. The finding supports the comments in the qualitative study by Thede et al. (1994) in which students remarked about the importance of having "technical" support available "to keep them from wasting valuable study time." However, this finding goes even further and indicates that participants from all three types of schools think that providing "instructional support" is equally as important as "technical" support to decrease the students' time. It is also interesting to note that the strategies were ranked higher than the other two strategies of "explain CAI" and “explain objectives," which could be used by teachers as a less expensive alternative to paying the on-going salaries of technical and instruction support personnel. 
The other finding of interest was that all three types of schools thought the use of the "small-group" and "whole class" modes of instruction were the two least "effective" strategies for decreasing the students' time. It was interesting because the BSN school participants rated both strategies lower than the AD schools and yet, BSN schools alone indicated they used both the "small group" and the "whole group" strategies "frequently." This finding raised the question that if the BSN school participants did not think the strategies were "effective" for decreasing the students' time then why did they use them "frequently?" The answer came through a comparison of the means for each of the four purposes which showed that the highest means, a 2.73 for "small-group" and 2.79 for "whole class," were both for student learning. The next highest means were also interesting since they showed a 2.70 mean for the "whole class" strategy for decreasing students' time and the other was a 2.70 for the "small-group" strategy and for decreasing the teachers' time. In conclusion, the findings seemed to indicate that the primary reason the BSN school participants used the strategies "frequently" were because they thought the increased student learning. The second reason the "whole class" strategy was used "frequently" seems to have been because they thought it saved the students' time and the reason they used the "small-group" strategy was because it saved the teachers' time.

\section{Comparison of Effectiveness to Decrease Teachers' Time}

The comparison of the three types of schools to determine how they rated the "effectiveness" of the 10 strategies for decreasing the teachers' time showed that BSN schools rated 4 and $\mathrm{AD}$ schools rated 3 of the strategies as "effective"while diploma schools did not think any of the strategies were "effective." The BSN schools perceived the use of including CAI content in a "course exam" was an "effective" way to save the teacher's time but AD and 
diploma schools did not. None of the schools thought the use of CAI to "replace traditional" instruction was an "effective" way to reduce the teacher's time. The findings also showed that public schools rated 3 and private schools rated 2 of the strategies as "effective." The third analysis showed that medium size schools perceived 4 and large schools thought 2 of the strategies were "effective"while small schools did not think any were. In addition to providing "instructional" and "technical" support, medium size schools thought an "individual" mode of instruction and using CAI to "replace traditional" instruction saved the teachers' time.

\section{Comparison of Correlations}

The findings of the first analysis of the strategies to increase student learning showed that out of 14 significant correlations for the BSN and AD schools, both schools had 7 strategies that also met the other criteria of being both "frequently" used and "effective." The 5 strategies the schools had in common were "explain CAI, explain objectives, motivate, integrate and courseexam." In addition, BSN had significant correlations for "required" and an "individual mode." Associate schools also had significant correlations for "follow-up" and "technical support." The comparison of the two institutions showed that out of 13 statistically significant correlations, public schools had 6 strategies and private schools had 5 that met all three criteria. The strategies they both had in common were "explain CAI, explain objectives, motivate, integrate, course-exam." Public schools alone also had a significant relationship for the strategy of "follow-up." The correlations for the three different sizes of schools showed that large schools had 8 statistically significant correlations, small schools had 6 and medium size schools only had 3 that also met the other two criteria. 
The analysis of the strategies to decrease costs showed that even though there were several statistically significant relationships, since none of the strategies were perceived to be "effective," even those that were "frequently" used failed to meet all three criteria and were eliminated. The correlations of strategies to decrease the students' time showed that 1 strategy, "explain CAI" met all three criteria for BSN schools and "explain objectives" met them for the AD schools. Again, 1 strategy, "explain CAI" met the criteria for the private schools while none met them for the public schools. The same strategy, "explain CAI" was only one that met the criteria for the small schools while "instructional support" was the only one that met the criteria for medium size schools and for the large schools, the single strategy was "small-group." The analysis of the strategies to decrease the teachers' time showed that out of 3 statistically significant relationships, only 1 strategy, "individual" mode of instruction met the other criteria for the BSN schools. For the AD schools, none of the 2 significant strategies that met the other criteria. None met all three criteria for the public or private schools and while there were 4 statistically significant correlations for the small schools, 2 for the medium size schools and 4 for the large, none met the other criteria.

In summary, the findings of the correlations only served to confirm the previous findings in showing that there was more agreement for the strategies to increase student learning than for the other three purposes. Once again, the same strategies of "explain CAI, explain objectives, motivate, integrate, course-exam, required" and an "individual mode" were the ones that were both "frequently" used and "effective" according to both BSN and AD schools. 


\section{Key Features of Schools}

Schools with a Computer Lab in the SON

Another avenue that was pursued in an effort to identify "key features" of the schools that effectively integrated CAI included an investigation of the number of computer resources that were available for students to use at the various schools.

The first section looked at the number and types of schools that had a computer lab in the school of nursing. Since there were no reports in the literature on this topic, the findings of this study provided new information. The results showed that twice as many, or $69 \%$ of the 109 schools had a lab in the school of nursing. Surprisingly, the figures showed that $68 \%$ of both BSN and AD schools and $100 \%$ of the diploma schools had a lab. The breakdown by the types of institutions showed that $71 \%$ of the public and $64 \%$ of the private schools had a lab. The findings for the different sizes of schools showed that $83 \%$ of all of the large schools, $68 \%$ of the small and only $60 \%$ of the medium schools had a lab in the school of nursing. The findings were surprising in light of the reports in the literature that indicated that BSN schools had more computer resources than AD schools. The fact that all the diploma schools had a lab was not surprising since the schools are typically private schools that are not located in a hospital setting. It was also surprising to see that more small schools had a lab than medium size schools. The fact that public schools had a lab more often than private fits with the idea of more financial resources being available to the public sector schools.

\section{Locations of Computers}

The next section described a comparison of the number of schools that reported having computers that students used for CAI in each of four different locations. The findings showed 
that of the 303 responses to the question, 29\% indicated their schools had computers in the SON, $29 \%$ said they had computers in the library, $21 \%$ in another department and $21 \%$ said they had students purchase their own. Once again, the findings are new to this study and so, no comparison could be made to figures in the literature. When the schools were broken down into CAI-user and non-user groups, the findings showed that $72 \%$ of the responses were from CAIuser schools as compared with only $28 \%$ from non-users. A larger number of AD schools reported having computers located in three departments (SON, another department and the library) but more BSN schools had students purchase their own computers. Most of all three types of schools reported the bulk of their computers were located in either the library or the SON. The differences between the two were minuscule.

The information provided by the responses to this question contributes little to the quest for the key features of schools that effectively integrate CAI. It is possible, however, to conclude from the findings that a larger number of schools that are CAI-users reported having computers in all four locations than schools that were not CAI-users and in all likelihood, it is probably the lack of computer resources that is one of the major reasons they don't use CAI. Also, the responses provide valuable information about the differences in resources of the various schools and the locations of the computers students use for CAI assignments. It seems pertinent that if the schools are to provide the "instructional support" that the participants indicated in their responses to the integration strategy question that they value so highly, then students working at computers that are located within the SON would logically have more access to personnel who are knowledgeable about nursing related questions than students working in departments outside the nursing department. MacKeller and Elliott (1999) observed that 
students who work at computers that are located in the library seldom have access to either technical or instructional support personnel and suffer from it. It seems apparent from the answers to this question that most of the schools of nursing in the U.S. have students use computers that are located in one of the two places and that the better of the two locations is within the SON, rather than the library or LRC unless the schools are able to provide the students with adequate technical and instructional support in those locations.

\section{Numbers of Computers}

The next question asked about the actual numbers of computers the schools owned. The purpose of this question was to determine which schools had the most computer resources and where they were located but also to compare the findings with those in the literature. It is important to differentiate the responses to this question from the preceding question. The previous question identified the number of schools that had computers located in the four locations but this question sought to identify the actual numbers of computers owned by the various schools and then identify where they were located. Since they were measuring two different things, the results to the two questions were very different but did not contradict oneanother.

The results of this study showed that all of the sample schools combined owned 12,265 computers $(\mathrm{M}=113)$. Clearly BSN schools owned most of the computers, or $55 \%$ followed by AD schools who owned 44\%. The diploma schools only owned 1\%. Carty and Rosenfeld (1998) conducted a survey study that was similar to this one in that their sample included 190 schools of nursing from across the U.S. and the respondents were a proportionate mix of diploma, AD, BSN and higher programs that were similar to those in the general population of schools of nursing in 
the U.S. A significant component of their survey addressed an inventory of technology resources available at each institution and the findings showed that almost all of the respondents (97.8\%) reported having computers available for students to use at their schools. They found that without exception, BSN and higher programs reported having more computer resources (i.e., computers, computers in faculty members' offices, a LAN, and Internet access) than diploma or associate degree programs. The results of their study showed that diploma schools had a mean of 14 , AD programs had a mean of 13 and baccalaureate programs had a mean of 30 computers (Carty \& Rosenfeld). In comparison, the results of this study showed that the mean for BSN schools was 52, and for AD schools, it was 31 and for diploma schools, it was 9 computers. The locations of the computers in this study were as follows: $50 \%$ were located in another department, $36 \%$ were in the library and only $14 \%$ were in the SON. As before, a large majority of the computers were owned by CAI- user schools. Most of the computers in the BSN schools were located in the library, followed by another department and a small percent were in the SON. Likewise, most of the computers in AD schools were located in another department and only a small percent were in the SON. Diploma schools had equal percentages of the few computers they owned in the SON and the library. In the Carty and Rosenfeld (1998) study discussed above, most of the computers were available in learning resource centers (58\%), nursing labs (47\%) and other locations (17\%), such as labs where students shared the computers with students from other departments.

There are obvious differences in the results of this study and those reported by Carty and Rosenfeld (1998). Perhaps the most telling results are the means for the different types of schools that have risen substantially over the ensuing years for all but the diploma schools. 
There is also a difference in the locations of the computers which sound as though they were primarily located in the library in the Carty and Rosenfeld study followed by almost half in the SON and a small percent in another department. In this study, a much lower percent of the computers were located in the SON which could be interpreted in one of at least two ways. The first is that the numbers of computers have increased so exponentially on the campuses in the ensuing years since that study that the percentages in the different departments have changed to reflect a much higher number, or it may mean that the trend is away from having computers within the department to having nursing students use computers that are located in large computer labs outside the department. In light of the responses to a previous question that showed that $69 \%$ of the AD and BSN schools and 100\% of the diploma schools currently have a computer lab within the SON, it seems the trend toward having a lab within the department has taken hold. However, it will be interesting to follow-up through future studies to see which direction the trend is actually taking.

\section{Technical and Instructional Support}

The next question looked at the types and numbers of technical and instructional support personnel the different types of schools employed. Again, the purpose was to establish which schools had the most computer-related resources that were available to the students. Based on the overwhelming responses when participants identified the most "frequently" used and "effective" integration strategies as providing technical and instructional support, the two strategies certainly stood out as an extremely important components for integrating CAI into the course. The findings showed that the schools employed more full-time than part-time employees and twice as many technical $(\mathrm{N}=469)$ as instructional $(\mathrm{N}=243)$ support personnel. The CAI- 
user schools employed 5 times as many technical and 9 times as many instructional support personnel as non-user schools. Even though BSN schools owned more computers, associate schools employed more of all four types of support personnel. The BSN schools did have a higher ratio of full-to-part-time technical and instructional personnel than either the AD or diploma schools. As before, public schools employed 5 times as many technical and 3 times as many instructional support personnel as private schools. The two types of institutions had the same ratio of full-to-part-time technical support employees but public schools employed a higher ratio of full-to-part-time instructional support personnel. Large schools employed more full-time technical and part-time instructional support personnel. Medium size schools employed more full-time instructional and small schools employed more part-time technical.

There are no previous studies or articles that address the topic of support personnel and therefore, a comparison with the literature was not possible. Perhaps the most obvious finding was the large discrepancy between the number of technical and instruction support personnel that are actually employed by the schools. This finding was not supported by the findings of this study that showed that participants rated the strategy of providing instructional support was equally as important as providing technical support. The finding that AD schools employ more support personnel was a surprise since BSN schools owned so many more computers. It seemed logical to conclude the same would be true of this resource. The fact that public schools employed more support personnel than the private was not a surprise since so many other findings have shown that private schools had fewer computer resources than public schools. The one finding that stands out is unusual was that fact that medium size schools employed more full-time instructional support personnel than large schools. One conclusion that can be drawn 
from the findings of this question is that $\mathrm{AD}$ schools provide students with more technical and instructional support, which based on the other findings of this study is an extremely important component when integrating CAI into the course.

\section{Key Features of Effective Educators}

The next section explored the personal characteristics (or demographic data) of participants in and effort to identify the key features of those who effectively integrate CAI into their courses. As mentioned previously, the sample $(\mathrm{N}=109)$ consisted of 45 participants from BSN schools, 59 from AD schools and 5 from diploma schools. It quickly became apparent from the findings to several questions in this study that $\mathrm{AD}$ and diploma school participants were much more active CAI-users than those from BSN schools. The percent of all BSN respondents who used CAI were only $56 \%$, while $81 \%$ of the AD and $80 \%$ of all of the diploma school participants were CAI-users. When the same data was analyzed by the type of institution, the results showed that of all the public school participants, $47 \%$ of the BSN, $85 \%$ of the AD and $100 \%$ of the diploma school participants in that group used CAI. Among the private schools, $73 \%$ of the BSN participants, $50 \%$ of the $\mathrm{AD}$ and $75 \%$ of the diploma school participants used CAI. Another analysis in which the participants were broken down by the size of the school showed that $83 \%$ of the small $\mathrm{AD}, 100 \%$ of the small diploma and only $36 \%$ of the small $\mathrm{BSN}$

school participants used CAI. Of the participants who worked for medium size schools, $68 \%$ of the $\mathrm{AD}, 100 \%$ of the diploma and $57 \%$ of the BSN school participants used CAI. Of the large schools, $69 \%$ of the $\mathrm{BSN}, 100 \%$ of the $\mathrm{AD}$ and $50 \%$ of the large diploma school participants used CAI. In summary, when the participants were divided into 6 different groups for analysis, the percent of BSN school participants were much lower for all except the private school group. 
The next section looked at the findings of the demographic questions on the questionnaire to see if by dividing the participants by their role, age, etc. helped to differentiated effective integrators (which ones used CAI most) from the others. The detailed findings were already discussed in a previous section and therefore, will not be repeated here but a brief overview of the findings will be included in the discussion. The findings of the breakdown by the participant's role showed that of the 97 participants who were faculty members, $72 \%$ used CAI. Of the 9 administrators, $67 \%$ used CAI and only one, or $33 \%$ of the 3 computer lab personnel indicated they used CAI. Of the 97 participants who were faculty members, those who used CAI most were from large, public and AD schools. Most, or $67 \%$ of the 9 participants who identified their role as administrators used CAI. Again, most of the administrators who used CAI were from large, public and $\mathrm{AD}$ schools. The single lab personnel participant who used CAI was from a medium sized, private, and BSN school.

The responses to the question about gender showed that $66 \%$ of the 6 males and $70 \%$ of the 102 females who participated in the study used CAI. Of the 6 males, those who used CAI most indicated they were from large, public $\mathrm{AD}$ schools. Of the females, the ones who used CAI most were from large, public AD schools.

A breakdown of the findings of the participant's age-ranges showed that $58 \%$ of the 7 participants in the first age-group of 20 to 40 -years, $65 \%$ of those in the 41 to 50 -year-old age group and $74 \%$ of those in the eldest, and largest age-group used CAI. For the 20 to 40 -year-old age-group, most were from small, public AD schools. For the 41 to 50-year-old- age-group, most were from large, public AD schools. For those who were 51-years and older, the ones who used CAI most were from large, public AD schools. 
A brief overview of the findings for educational preparation showed that for the participants in the BSN and lower group, $86 \%$ used CAI. For the MSN group, $63 \%$ used CAI and for the Ph.D. group, 79\% used CAI. The participants from the BSN or lower educational preparation group who used CAI most were from small, public AD schools. Most of the participants with an MSN degree who used CAI were from small, public AD schools and most with a Ph.D. who used CAI were from large, public and AD schools.

In summary, the evidence is strong and clearly indicates that the most "effective" integrators of CAI in all but 4 the above categories are from large, pubic AD schools. A summary of the findings showed that the results of 11 analyses indicated participants from large schools outnumbered small schools by 8 to 3 , public schools outnumbered private schools by 11 to 0 and participants from AD schools outnumbered BSN by 10 to 1 . The findings also showed that the participants who identified themselves as "faculty members" indicated their schools used CAI (72\%) more often than the administrator respondents (67\%) and much more often than the computer lab personnel (33\%). Female participants (70\%) also came from schools that used CAI more often than the male participants $(66 \%)$. Perhaps the most surprising response was that the participants from the 20 to 40 -year-old age-group used CAI less than participants in the 41 to 50 year-old age-group (65\%) and much less often that participants in the 51-year and older age group (74\%). It was also interesting to note that participants who had a BSN and below degree educational preparation used CAI more (86\%) than the Ph.D. participants (79\%) and much more often than the MSN participants (63\%) who made up the bulk of the respondents. In conclusion, based on the responses to this question, the educators that are the most effective at integrating CAI have the characteristics of working for large, public AD schools and are faculty members 
Integration of CAI 330

and not administrators or computer lab personnel, they are females and in the 51 and older age group and have a BSN degree or lower, rather than an MSN or Ph.D.

Less Concrete, but Interesting Key Features of Schools

The next section examined some of the less concrete key features of schools in order to identify some of the characteristics of those schools that were more "effective" at integrating CAI. As described in the discussion of the results, the analysis consisted of a simple comparison of the schools that had some "feature" or had a specified number of the "feature" against the others who did not. The statistical analysis consisted of calculating the means for the participants' responses to Questions 2 through 6 of the ICAI Questionnaire for each group and then comparing to see which group had the most means that were higher. The purpose was to link the key features of the schools with the participants' ratings of "frequency" and perceptions of the "effectiveness" of the strategies to see if the feature made a difference.

It will be recalled that the findings of the first comparison showed that schools that had a computer lab in the SON had 46 means that were higher as compared to 15 higher for schools that did not have a lab. The next inquiry was closely related and showed that schools that had computers in the school of nursing used the strategies more and perceived them to be more effective for student learning, decreasing the students' time and deceasing the teachers' time but not for decreasing costs (50 to 12). The findings of the next part showed that schools with computers in another department used the strategies more and rated them as more "effective" for all four purposes than schools that did not have computers in another department (44 to 17). The next part looked at schools with computers in the library, but this time, the results showed that schools that did not have computers in the library had a greater number of means that were 
higher in all five areas and were unanimous for the strategies for decreasing costs and decrease the teachers' time (15 to 48 ). The fourth inquiry compared the schools where students purchased their own computers against those that did not and the findings showed that they rated the strategies as more effective in all areas (51 to 11) except "costs" where there was a tie.

The results showed that, with the exception of the schools with computers that were located in the library, the schools that had a computer lab and those that had computers in each of the 4 locations used the strategies much more "frequently" and perceived the strategies were more "effective" than schools that did not have a lab or computers in those locations. While it is hardly surprising to learn that schools that had computers did better than schools that did not, the findings of this section showed that the locations of the computers may well have made the difference in the "frequency" of use and perceptions of the "effectiveness" of the strategies. In general, schools with a computer lab and computers in the SON used the strategies more "frequently" and thought the strategies were more "effective" for increasing student learning than schools with computers in the other locations. Participants from schools where students purchased their own computers rated the strategies for decreasing costs, students' and teachers' time as more "effective" than participants who had computers in the other four locations, including those that had computers in the SON and had a computer lab. The findings make sense since the major reasons schools have students' purchase their own computers are to decrease costs as well as the teachers' and the students' time (Massy \& Wilger, 1998). Whether or not that actually happens is another issue, but the thinking is that it will. MacKnight (1995) noted the trend in higher education has been toward the decentralization of computer departments so that more computing would be done at the departmental level. Another facet of 
the decentralization process was that centrally located computer labs were expected to disappear as college students purchased their own personal computers and mobile lap tops and began accessing the schools' software and programs that are being made available over the Internet from anywhere, anytime (MacKnight).

The next section compared schools that had a specific number of computers in three different locations with schools that had fewer than that number in the same location. The findings reinforced the findings of the previous section but answered the question from a slightly different perspective. The differences between the number of strategies that were used by the schools that had the feature were just as impressive as the last group of figures but instead of just identifying a location, they showed that it was the location and not the number of computers at the location that made a difference. For instance, the comparisons measured the differences between schools that had "15 or fewer computers" with schools that had "16 and more" computers in the SON, the next compared schools that had "30 or fewer" computers in another department with schools that had "31 or more," and the last group compared schools that had "24 or fewer" computers in the library with schools that had "more than 25." The differences between number of strategies that were higher for the groups were " 32 " for the SON, "13" for schools with computers in another department and a difference of " 20 " for schools with computers the library. In other words, the findings showed that as the numbers of computers in each location increased, the number of strategies that separated those "with" the feature from those "without" it decreased. The real significance of the findings was that it suggests that it was not the number of computers in the location, but rather the location it-self that made the difference in the participant's use and perceptions of the "effectiveness" of the strategies. 
Another obvious finding that leaped off the page from this series was that schools that had computers that were located in "another department" had 8 strategies that were higher for increasing student learning, schools that had computers in the library had 9 that were higher and those that had computers in the SON had 17 higher strategies. Once again, the evidence from both parts of the question points to the location of the computers as being an important key feature of schools that are more "effective" at integrating CAI and once again, they showed that schools that had computers in the school of nursing had did more than participants from schools with computers in either of the other two locations.

The next part of the inquiry looked at the number of full and part-time technical and instructional support personnel employed by the school to see if those types of "human" resources made a difference in the "frequency" of use and perceived "effectiveness" of the strategies by participants. The first analysis examined the number of technical support personnel and the findings showed that schools that employed " 3 or more full or part-time technical personnel" had more means that were higher than schools with "less than 2 " (8 to 53). The findings of the second part of the question showed that schools that employed " 2 or more instructional support personnel" also had more means that were higher than schools that employed fewer employees (14 to 49 ). The third inquiry combined the two types of support personnel and the findings showed that the schools with " 4 or more technical and instructional support personnel" had more means that were higher than schools that had less than 4 (10 to 53).

To summarize, the findings seem to indicate that participants who worked for schools that employed larger numbers of technical and instructional support personnel used the integration strategies much more "frequently" and perceived the strategies to be much more 
“effective"for all four purposes than participants who worked at schools that employed fewer support personnel.

The final analysis in this section examined the percent of teachers at the school that used CAI to see if the percent of teachers who were CAI-users made a difference in the "frequency" of use and perceived "effectiveness" of the strategies. It may be recalled that the findings to Question 18 B of this study showed that $72 \%$ of the respondents indicated that " $50 \%$ or less" of the faculty at their school used CAI, while at the other end of the spectrum, $25 \%$ of the schools indicated that between " 80 to $100 \%$ " of the teachers were CAI-users. The findings of the first part of the analysis showed that the means for schools where " $41 \%$ or above" of the faculty used CAI actually used the strategies less and perceived they were less "effective" than schools where less than $40 \%$ of the teachers were CAI-users (35 to 27). However, the second part of the study showed a very different response in which schools where " $51 \%$ and above" of the faculty used CAI had more means that were higher than schools with " $50 \%$ and lower" (28 to 37 ). A closer look showed that while the percent of faculty who used CAI had little bearing on the "frequency" of use or the perception of the effectiveness of the strategies for decreasing costs or the students' time, there was a difference in the way the participants perceived the “effectiveness" of the strategies for increasing learning and decreasing the teachers' time. Therefore, it seems that it would be safe to say that teachers who work at schools where more than $50 \%$ of the teachers use CAI use the integration strategies more "frequently" and perceive they are more "effective" for increasing student learning and decreasing the students' time. It might be a stretch to infer from these findings that since only $28 \%$ of the teachers in the United States work at schools where $51 \%$ or more of the teachers at their schools are CAI-users, most 
are not working for schools that are "effectively" integrating CAI.

This group of inquiries certainly resulted in some interesting observations and generated almost as many questions as were answered. One finding, in particular, that was perplexing was the one that showed that schools that did not have computers in the library used the strategies more often and perceived them to be much more "effective" for learning, decreasing costs and the teachers' time than schools with computers in the library. The results ran contrary to all the other responses and therefore, must be called into question since the very next question showed that schools with "25 or more" computers in the library used the strategies more and perceived them to be more effective than schools with " 24 or fewer"computers in the library. The only explanation for the discrepancy between the two findings is that the answers came from a different mix of participants when the data was sorted. Even still, the validity of the response is suspect and until they are verified, should not be taken seriously.

There were several findings that were of particular interest in the section of inquiries that showed that participants from schools with a computer lab in the SON and those that had computers that were located in the SON rated 17 strategies higher for the "frequency" of use and all 19 of the strategies for "effectiveness" for increasing student learning higher than schools that did not have computers in the SON or a lab. The findings suggest the participants that worked in schools that had computers within the nursing department were more active integrators that those who did not. In light of the findings in the literature that the trend is away for centralized banks of computers in laboratories toward decentralization and moving smaller numbers of computers back into each department or school, these findings provide support for such a move and could help to contribute to the argument in favor of it. 
Integration of CAI 336

It was interesting to note the differences between the schools that had students purchase their own computers and those that did not. There was no difference in the strategies for "costs" but the strategies to decrease time were thought to be more effective. The findings for the costs may have been low because many of the strategies simply did not apply to students using CAI from home. For instance, the only mode of instruction that would apply would be "individualized." Making the CAI a "required" part of the course and using it to "replace the traditional method of instruction" would be part of the course rather than strategies the teacher would use or not use with each course. Actually, the only two strategies that would significantly impact the cost of the course with students working from home would be the need to provide "technical and instructional" support. However, the participant's responses that having students purchase their own computers saved both the students' and the teachers' time were consistent with reports in the literature. All of the findings are interesting fodder for further studies.

Is summary, the findings from this part of the study indicated schools that have students purchase their own computers were more effective at integrating CAI than schools that do not and than schools that have computers located in another department. Schools that have students purchase their own computers were also more effective at integrating CAI than schools that have a computers or a lab in the SON. Schools that have computers in the department were more effective at integrating CAI than schools that have computers located in another department. They also used the integration strategies more frequently and perceived the strategies for student learning were more "effective" than schools that have students purchase their own computers.

While these findings are hardly conclusive evidence, they could be a wonderful starting point for further studies in the area of educational productivity in higher education. 
They also used the integration strategies more frequently and perceived the strategies for student learning were more "effective" than schools that have students purchase their own computers.

While these findings are hardly conclusive evidence, they could be a wonderful starting point for further studies in the area of educational productivity in higher education. They also have serious ramifications for the quality of education not only for nursing but for education in general. One spin-off study that comes immediately to mind is an investigation to see how many of the strategies teachers working with students from home use as compared to teachers working with on-campus students. It would be interesting to see how the distance affects the teachers' awareness of the students' need for support and direction when the student is given a CAI assignment. Another might be to compare the students' perceptions of the effectiveness of the strategies as compared to the teacher's. A very basic study might measure the differences in the strategies that are used by teachers who have a computer lab in the SON, or the Department of Engineering, or the science department, as compared with teachers that do not have a lab or computers within the department and send the students off to another department to do the assignment. It's almost like comparing parents who home school against those who send their children off to school.

Also schools that employed more technical and instructional support personnel were more effective at integrating CAI than schools that employed fewer numbers. The same conclusion can be drawn from the next findings and the section that looked at the percent of teachers who were CAI-users. Schools that had $51 \%$ or more of the teachers who were CAIusers were much more effective at integrating CAI than schools that had lower percentages. Here again, there also seemed to be a need for a "critical mass" or percent of the faculty who 
Integration of CAI 338

used CAI in order to have that synergistic effect spoken of by Cambre and Castner (1993). One interesting segment of the finding was that participants who worked for a school where $51 \%$ or more of the teachers used CAI indicated they perceived the strategies were more "effective" for "increasing student learning" and "decreasing the students' time" than the others. It will be recalled that several of the instructional design theories stated that the two almost always go hand-in-hand and the same strategies that increase learning almost always also decrease the students' time. This finding certainly reinforces that assumption.

\section{Types of Software}

The initial answers to the fourth study question showed that the participants reported using 5,540 software programs during the previous year and of those, $41 \%$ were tutorials, $34 \%$ were drill-and-practice, $19 \%$ were simulations, $4 \%$ were virtual reality programs, and $2 \%$ were expert systems. As expected, these findings differed considerably from the most recent, but extremely dated reports in the literature in which Bowell (1995) described the software as having evolved from primarily text-based case-study type simulations and a small number of tutorials in 1993 into $20 \%$ graphic simulations and 6\% tutorial programs by 1995 . Based on the results of this study, it seems the tutorials may have overtaken the case-study type simulations during the ensuing years. The findings of this study also differ from other dated information in the literature that listed text-based "simulations" as the most often used type of software followed by tutorials, drill-and-practice, and testing programs (Mikan \& Aiken, 1992). The findings of this study support the reports by several authors who described the increasing use of graphic simulations and virtual reality software programs in a variety of specialty fields (Cheek et al., 1998; Kilmon, 1996; Lyons and Milton (1999); Theile \& Holloway, 1990; White, 1995). Also, 
Integration of CAI 339

while the number of "expert systems" software programs being used in nursing education and to teach and provide professionals with on-the-job decision making support (Hanson et al., 1994; Jirapaet, 2001; Martin, 2001) have increased substantially over recent years, the percent of the total has remained almost the same as it was when Bowell reported on it in 1995.

The sheer volume of the software programs that were used over the previous year seems to indicate that schools are using older as well as newer versions of software since the literature indicated that the number of new software titles began declining around 1993 and 1995 (Bowell,1995). Edwards (1995) noted that the number fell from 700 to 300 titles during that period which she attributed to a trend in the industry in which commercial software companies and branches of the major nursing textbook publishing companies drove the small, independent designers and companies out of business when they began producing high quality graphic tutorial and simulation programs.

One surprising finding in this section was the fact that AD schools used more of all five types of software than BSN or diploma schools. It was assumed before the study began that BSN schools would have more computer resources and that they would include all types of resources including software. The assumption was based on the several reports in the literature and yet, that certainly did not turn out to be the case in this study and particularly not in the area of software use. The actual figures were $78 \%$ of the programs were used by AD schools, $21 \%$ were used by BSN and the number for diploma schools were so small the percent was $0 \%$. It was disappointing to see that public schools used almost 17 times as many software programs as private schools. However, since BSN schools made up the majority of the private schools, the reports of low use were evident in both figures. Public schools used $94 \%$ of all the programs and 
private schools only used $6 \%$. As expected, large schools used over one half of all the programs, medium schools were next and small schools used the least. Associate schools used more drill-and-practice programs and tutorials while BSN schools used mostly tutorials. It was noted in the definitions of terms at the back of the ICAI Questionnaire that the decision to include drill-and-practice programs on the list of five types of software for teaching decisionmaking was made because students use them to practice basic mathematical drug calculations, which it was reasoned, could be linked to the clinical decision-making process. However, the fact that drill-and-practice programs were being used so extensively in nursing education and to the point that they were the second most extensively used program was unexpected.

The second part of fourth study question looked at the number of commerciallypurchased or in-house produced software programs used by the schools. Out of a total of 2,312 programs, almost all were commercially-purchased. Of the commercially purchased programs, AD schools used 56\%, BSN schools used $43 \%$ and diploma schools only used $1 \%$. Once again, AD schools used more of all three types of software. Bachelor schools used more of the few customized programs and AD schools used more teacher-authored programs. Public schools used ten times as many programs as the private schools. Medium size schools used more of the programs than large schools and twice as many as the small schools. All three sizes of schools primarily used commercially-purchased programs. The fact that BSN schools used more customized programs corresponds to the fact that BSN schools also had larger numbers of computers than AD or diploma schools. Several authors noted that customized software was by far the most expensive type of software because it is developed by a team of specialists who are either employed by the school or work as consultants (Bates, 1999). 


\section{Peers' Ratings of Effectiveness}

The fifth study question looked at how participants rated their peers for "effectiveness" at integrating the various types of software into the courses they taught. Here again, there are no studies in the literature that address the topic and so these findings will be the first published findings against which others can be compared. The findings did show that, as a group, the participants thought their peers were most effective at integrating tutorials, and then simulations, followed by drill-and-practice, virtual reality and then, expert systems. The results of the analysis by the types of schools showed that $\mathrm{AD}$ participants rated their peers as being more "effective" at integrating all five types of software. Participants from AD schools indicated their peers were most effective at integrating "tutorials" and BSN participants thought their peers integrated "simulations" most effectively. Both AD and BSN schools rated their peers as least effective at integrating "expert systems" programs.

When the participants rated the teachers at their schools on how effectively they integrated commercially-purchased and in-house produced software, the findings showed that not only was commercially purchased software used much more "frequently" but participants also thought the teachers at their schools were much more "effective" at integrating it. As before, AD school participants rated the teachers at their schools as more "effective" at integrating all three types of software than BSN participants. Both types of schools thought they integrated commercially purchased software best. The findings contradict some of the strong recommendations in the literature that teachers were better able to integrate "teacher-authored" and "custom-produced" software than commercially-produced. The vast numbers of commercially-produced programs that are being used may have skewed the findings in that 
direction. It may also have simply been that very few participants had ever worked with customized or teacher-authored software that was the deciding factor.

The correlations were not much value in this part of the study since the results only showed strong relationships between the types of software that were seldom used and were not perceived to be integrated "effectively" by the participant's peers.

\section{Conclusions}

\section{Most Important Findings}

The first, and in my opinion, most important finding of the study was the fact that AD schools and teachers used CAI so much more often than BSN schools and teachers. The ramifications of the findings are numerous, especially in light of the fact that BSN schools owned so many more computers than AD or diploma schools. The mean for BSN schools was much higher than the other two and the finding was actually an increase from the figures that were in the most recent article on the topic (Carty \& Rosenfeld, 1998). So, while the schools have continued to purchase greater numbers of computers, their use is far behind what their contemporaries in the AD schools are doing. Perhaps the most impressive of the findings was the comparison of the percent of faculty who used CAI that showed 27 of the AD school participants reported " 80 to $100 \%$ " of the teachers at their schools used CAI as compared to only 3 from BSN schools. The AD schools also used many more times the numbers of software programs, larger numbers of commercially-purchased and customized programs, provided greater numbers of both technical and instructional support and they rated their peers as more "effective" at integrating all types of software than the BSN schools. In all, the findings of almost every section of this study showed that AD teachers and schools were the champions of 
CAI which was an enormous surprise to this researcher and I'm sure will be to the nursing education community.

The second most important finding was the fact that BSN school participants used so many more strategies "frequently" than AD or diploma schools. One possible reason for the finding may be a work environment where they are encouraged to be innovative with teaching strategies and to take more risks, such as using some of the strategies that, according to the experts in the field, are the most "effective" for improving all four measures of productivity (Bates, 1999). It will be recalled, some of the strategies BSN school participants used "frequently" included "using CAI to replace the traditional method of instruction, and using a small group and whole-class mode of instruction." In other words, the strategies BSN schools used "frequently" were the ones that were evaluated by the experts as some of the most "effective" for improving educational productivity in all of its many facets and not just to increase learning (Bates, 1999). On the other hand, AD and diploma school participants indicated they used the "optional" strategy "frequently" which was possibly the least effective strategy of the nineteen and that has been shown to have a negative impact on costs, as well as time (Bates, 1999). In conclusion, while the BSN and AD participants' ratings of the strategies were almost exactly the same for "effectiveness," the strategies the BSN schools used "frequently" were more "effective" strategies. Therefore, while AD schools turned out to be the champions of CAI and provided their students with a better environment for its optimal use, BSN schools used the right integration strategies more "frequently" and as a result, more "effectively." 
Another important, and related finding were the strength and consistency of the results that showed the participants "frequently" used and perceived the exact same strategies were "effective" for student learning and yet, didn't think any were "effective" for decreasing costs or that only a few were effective for the other purposes. The findings suggest the participants didn't relate as well to the other three educational productivity measures as they did with student learning. One comment in the "Participants' Comments" section of the study (See Appendix E) read, "I truly hope you are not finding schools who use CAI's to decrease the time they spend with students!!” The comment almost encapsulates the same values as the ones that are suggested by the findings and an attitude that there is something wrong with using CAI for anything other than to increase student learning, including cutting down on the time it takes for the student to learn. There were many other insightful and inspiring comments that respondents made that are also included in the "Comments Section" that would make an excellent report on its own because it provides an inside view of what is really happening out there in the real world of nursing education. But still, it is true that there are still many teachers who think that the only effective teaching comes from a face-to-face encounter with an instructor and nothing else will substitute for the teacher "telling" the students what is right and wrong. Such a viewpoint fails to grasp the value of having students actually interacting and making decisions rather than hearing about or observing others making them.

Another explanation for the finding might be the way the questionnaire was worded or organized. Perhaps the wording or the organization of the questions in the questionnaire influenced the way participants valued the other strategies simply because student learning was placed first and the others followed. A second, follow-up study might invert the order of the 
strategies or place the questions, or even the strategies in a different order to see if participants respond differently.

\section{Unanticipated Occurrences}

There were several unanticipated occurrences that happened during the study. The first was an unexpected problem that could have easily been avoided by the researcher, but was not due to lack of experience. The problem occurred during the initial sign-up period when participants were being recruited for the study. Instead of waiting until all of the participants were signed up before sending out the cover letters, it would have been better to send the cover letter as soon as the participant agreed to take part in the study. The response rate would have been dramatically improved and the target number of 140 participants would have been reached had it been done that way. Unfortunately, several participants who initially agreed to complete the questionnaire got busy or had other things happen and did not take part in the study.

A second unexpected occurrence was that during the "Prospectus Proposal Meeting" it was agreed that the questionnaire should include a question about the participants' years of teaching experience. There are many references in the literature that relate more teaching experience with better quality teaching and higher levels of expertise with computer-use. Unfortunately, the question was inadvertently omitted from the questionnaire and the fact that it was missing did not come to the attention of the researcher until several responses had already been returned. The information that might have been collected from the question can be inferred from the ages of the participants and the levels of use of CAI but, had it been included, it would have provided additional and valuable information. 


\section{Unanticipated Findings}

The findings of the analysis of the frequency of use of the strategies by the entire group $(n=77)$ showed that 29 of the 44 strategies, or $66 \%$ were "frequently" used. They included 11 of the 19 strategies to increase student learning, 4 of the 8 to decrease course costs, 5 of the 7 to decrease the students' time and 4 of the 10 to decrease the teachers' time. At first glance, it appeared that more strategies were used for the purpose of increasing student learning than any of the others. However, a second look at the data showed that a higher percent of the strategies that were "frequently" used were the strategies to decrease the students' time (71\%), followed by strategies to increase student learning (58\%), which were followed by strategies to decrease costs $(50 \%)$ and finally, by strategies to decrease the teachers' time (40\%). One possible explanation for this finding might be that many teachers have an aversion to looking for ways to decrease the time they spend teaching and on teaching-related tasks, such as test construction, administration, etc. and value the strategies that are intended to save the student's time more highly simply because they seem more altruistic. Another explanation might be that teachers, just as most people, are resistant to change and prefer feeling in "control" rather than turning the teaching role over to a technology.

Another unanticipated finding was that the participants consistently identified the strategies of providing "technical" and "instructional"support as not being effective for reducing costs, but as the two most "effective" strategies for each of the other three purposes. Numerous recommendations for the using the strategies appeared in the literature but, based on the findings of the only study that addressed the topic, Thede et al, (1994) found that a lack of technical support personnel was one of the major problems the students identified. Another surprise was 
that none of the strategies for decreasing costs were perceived to be "effective"for that purpose. The fact that none of the schools thought using CAI to "replace traditional" instruction was "effective"for reducing costs is a mystery that deserves further exploration. The fact that they

used the "required" strategy more often than assigning CAI as an "optional" learning experience was encouraging, but was also unexpected.

\section{Recommendations}

\section{Recommendations for Nursing Education}

- One recommendation based on the findings of this study would be to consult with commercial-software-developers to explain how they might increase the educational productivity of their software by designing it so that teachers could use the "embedded" tests to evaluate student learning. It would mean designing the software with adequate security and record-keeping capabilities so that the teacher could download the test results simply and easily into an electronic grade book. It just makes sense that learning would increase if students knew they were being tested and graded on tests that were intersperse throughout the program. It would certainly leave the students with the impression that the CAI assignment was an integral part of the course and worth their time and effort. The strategy would save the students' time because the information would not need to be tested again on an in-class examination. It would save the teachers' time by eliminating the need to construct, administer and evaluate test questions. Another advantage would be to improve learning by taking advantage of the unique capabilities of the computer to test the students' decision-making abilities rather than just asking them to describe how it was done. The strategy would also 
make it possible for the teacher to easily conduct a summative evaluation of learning and make those "anticipated" major and minor changes to the course in a timely fashion based on the students' performances. One more advantage secure "embedded" testing capabilities would provide is that the teacher would have access to specific information about which students did and did not meet the objectives using the CAI program so that some form of "alternative" learning method could be recommended or provided. The fact that students would not be tested again on the content of the program would not mean that the teacher would not, or should not conduct some type of post-instruction discussion with the class or small-groups of students in a clinical setting. Also, by devising follow-up activities, the teacher would provide the students with a chance to gain additional insights into why their answers were wrong and to correct misconceptions. Both post-instruction strategies would provide the teacher with an opportunity to evaluate the value of the program and provide the extrinsic feedback that is so important for students to integrate and transfer what was learned from the CAI program into the rest of the course.

- Teachers should become more innovative when designing courses that include CAI so that all four measures of productivity are improved. They need to stop focusing so narrowly and recognize the other three purposes for using CAI are as valuable as increasing learning. Teachers in any department, but particularly nursing education, need to begin using the types of CAI programs that provide students with practice making clinical decisions and use it to "replace" some, but not all face-to-face teaching.

- Recognize the implications of the findings that showed that BSN educators have so 
many more computers and yet are being badly outdone by their colleagues in the associate degree programs.

- One recommendation for associate degree teachers is to continue with all the strides they have made in using CAI as a teaching tool but to use the strategies more "frequently" and try using some of the more innovative strategies such as using CAI for small-group and whole class presentations.

- Be creative in finding ways to assess the students' learning when they do a CAI assignment. Remember, they will not do it if it is designated as an "optional" assignment.

- Begin pretesting the software before purchasing it in a way that students in the course have input into the decision. In other words, match the software to the students who are taking the course rather than purchase software just because it teaches a particular topic.

- $\quad$ Become discriminating customers when purchasing software and talk to software sales people about what you want the programs to do. Remind them you are interested in saving your own time as well as the students'.

- Further studies should be done to determine the validity of the findings that schools that have computers in the department and that had students purchase their own computers were so much more effective at integrating CAI than the others.

\section{Recommendations for General Education}

- There are numerous implications in this study for teachers at every level of education. For instance, the literature was full of reports of teachers who identified the need for 
Integration of CAI 350

more information on how to integrate CAI programs into the course. The list of 19 strategies, while certainly not all-inclusive, is a good place to begin. Many of the findings are applicable to any school setting such as which strategies were perceived to be effective and the ones that were frequently used.

- A recommendation for administrators in higher education is to move the computers as close to the department as possible. The findings of this study strongly suggested that schools that had computers within the department also had teachers who used the strategies more often and perceived they were more effective than those who did not. A major finding of this study was that the participants used the exact same strategies frequently that they perceived were effective for increasing student learning. Turned around, that statement could also be interpreted to mean the more frequently the strategies were used the more the students learned.

- The findings of the types of software the schools were able to integrate most effectively should give educators insight into which types are best for integration purposes.

- One follow-up study that is recommended is to explore the question of which strategies might be "effective" for decreasing costs when using CAI. The answer would be extremely helpful in light of the fact that none of the strategies in this study were thought to be effective. It is also a timely question since educational productivity has become a major consideration with all types of instruction.

- Another study might look at the question about a "critical mass," or percent of faculty who used CAI and what impact that might have on how teachers perceive the 
Integration of CAI 351

effectiveness of the strategies. Another good study question might look at the impact of having a computer lab in the SON on the students' and the teachers' time, as well as student learning and costs. While it is widely recognized that one school's expenses cannot be compared with another, learning which strategies decreased costs would be a different question all together. 


\section{REFERENCES}

Abrams, A., \& Streit, L. (1986). Effectiveness of interactive video in teaching basic photography. Technological Horizons in Education Journal, 14, 92-96.

Abdulla, A.M., Henke, J., \& Watkins, L. (1987). Computer-aided learning: Experienced perspectives and promises. Journal of American Cardiology, 9(3), 678-683.

Adams, C., Murdock, J. E., Valiga, T. M., \& McGinnis, S. (2002). Trends in registered nurse education programs: A comparison across three points in time- 1994, 1999, 2004. National League of Nursing. Retrieved October 10, 2002, from www.nln.org/aboutnln/ nursetreands.htm

Alessi, S. M., \& Trollip, S. R. (1985). Computer-based instruction: Methods and development. Englewood Cliffs, NJ: Prentice-Hall.

Anderson, J. R. (1990). Cognitive psychology and its implications. (3rd Ed). New York: W. H. Freeman and Co.

Angel, B. F., Duffey, M., \& Belyea, M. (2000). An evidence-based project for evaluating strategies to improve knowledge acquisition and critical-thinking performance in nursing students. Journal of Nursing Education, 39(5), 219-28.

Arnold, J. M. (1990). Development and testing of a student software evaluation form for computer-assisted instruction. In J. M. Arnold \& G. A. Person (Eds.). Computer applications in nursing education and practice. New York: National League of Nursing Publications. 
Austin, S. L. (1999). Baccalaureate nursing faculty performance of nursing computer literacy skills and curriculum integration of these skills through teaching practice. Journal of Nursing Education, 38(6), 260-8.

Bailey, P. (1994). Computer use a common thread for teaching winners. Dateline, University of California, Davis. 7(18), 1.

Baird, W. E., \& Koballa, T. R. (1988). Changes in pre-service elementary teachers' hypothesizing skills following group or individual study with computer simulations. Science Education, 72, 209-223.

Bal, A., Britnell, J., McCarthy, C., \& Samuels, S. (1995). Evaluation of an interactive multimedia application to learn interpersonal skills. Medinfo, 8(2), 1694.

Bareford, C.G. (2001). Community as client: Environmental issues in the real world a SimCity computer simulation. Computers in Nursing, 19(1), 11-16.

Barrows, H.S. (1983). Problem-based, self-directed learning. JAMA, 250(22), 3077-3080.

Bass, J.D. (1991). Factors that relate to the diffusion and adoption of computer-assisted instruction among collegiate nurse educators. (Doctoral dissertation, University of Georgia, 1991). Dissertation Abstracts International, AAT 9133454.

Bates, A.W. (1995a). Technology, open learning and distance education. London: Routledge. Bates, A.W. (1995b). Creating the future: Developing a vision in open and distance learning. In F. Lockwood (Ed.), Open and distance learning today. New York: Routledge. 
Bates, A.W. (1999). Restructuring the university for technological change. In J. Brenner, J. Fedrowitz, M. Huber, \& T. Shah (Eds.). What kind of university? International perspectives on knowledge, participation and governance. Philadelphia, PA: The Society for Research into Higher Education \& Open University Press.

Becker, H. J. (1994). How exemplary computing-using teachers differ from other teachers: Implications for realizing the potential of computers in schools. Journal of Research on Computing in Education, 26(3), 291-321.

Bejerano, Y. (1987). A cooperative small-group methodology in the language classroom. Teachers of English to Speakers of other Languages Quarterly, 21, 483-504.

Belfry, M. J., \& Winne, P. H. (1988). A review of the effectiveness of computer-assisted instruction in nursing education. Computers in Nursing, 6(2), 77-85.

Benner, P. (1984). From novice to expert: Excellence and power in clinical nursing practice. Redwood City, CA: Addison Wesley Nursing.

Benton, D.C. (1989). Evaluating computer-assisted learning: A necessity for nurse education. Information Technology in Nursing, 1(1), 7-9.

Bersky, A. K., \& Krawczak, J. (1995). Building a nursing activity database for processing freetext entry during computerized clinical simulation testing. Computers in Nursing, 13(5), 236-243.

Bertalanffy, L. V. (1968). General systems theory: Foundations, developments, applications. New York: G. Braziller.

Bitzer, M., \& Boudraux, M. C. (1969). Using a computer to teach nursing. Nursing Forum, 8, 234-254. 
Bloom, K.C., \& Trice, L. B. (1997). The efficacy of individualized computerized testing in nursing education. Computers in Nursing, 15(2), 82-88.

Boettcher, E. G., Alderson, S. F., \& Saccucci, M. S. (1981). A comparison of the effects of computer-assisted instruction versus printed instruction on students learning in the cognitive categories of knowledge and application. Journal of Computer-Based Instruction, 9(3), 98-107.

Boney, J., \& Baker, J.D. (1997). Strategies to teach clinical decision-making. Nurse Education Today, 17(1), 16-21.

Bolwell, C. (1993). Directory of educational software $\left(5^{\text {th }}\right.$ ed.). New York: National League for Nursing. Athens, OH: Fuld Institute for Technology in Nursing Education.

Bowell, C. (1995). Index to computer-assisted instruction. In M. J. Ball, K. J. Hannah, S. Newbold, J. Douglas (Eds.), Nursing informatics: Where caring and technology meet ( $2^{\text {nd }}$ Ed.). New York: Springer-Verlag.

Bolwell, C. (1998). Evaluating computer-assisted instruction. In V. K. Saba; D. B.Pocklington; \& K. P. Miller (Eds.), Nursing and computers: An anthrology, 1987-1996. pp. 574580, New York: Springer-Verlag.

Bouton, C., \& Garth, R. Y. (Eds.). (1983). Learning in groups. Washington, DC: Jossey-Bass. Bratt, E., \& Vockell, E. (1986). Using computers to teach basic facts in the nursing curriculum. Journal of Nursing Education, 25, 247-251.

Bryson, D.M. (1991). The computer-literate nurse. Computers in Nursing, 9(3), 100-107. Briggs, L.J. (1991). Instructional design: Principles and applications. Englewood Cliffs, NJ: Educational Technology Publications. 
Integration of CAI 356

Brudenell, I., \& Carpenter, C.S. (1990). Adult-learning styles and attitudes, and modes of instruction. Journal of Nursing, 3, 164-170.

Budacki, R. (1992). The effects of computer-assisted instruction versus traditional lecture method on associate degree nursing students' cognitive achievement and attitude toward learning. (Doctoral dissertation, Duquesne University School of Nursing, 1992). Dissertation Abstracts International, AAT 1349810.

Bunderson, C.V., Baillio, B., Olsen, J. B., Lipson, J. I., \& Fisher, K. M. (1984). Instructional effectiveness of an intelligent videodisc in biology. Machine-Mediated Learning, 1, 175215.

Calderone, A.B. (1994). Computer-assisted instruction: Learning, attitudes and modes of instruction. Computers in Nursing, 12(3), 164-170.

Carty, B., \& Rosenfeld, P. (1998). From computer technology to information technology: Findings from a national study of nursing education. Computers in Nursing, 16, 259-265.

Cambre, M., \& Castner, L. J. (1993, March). The status of interactive video in nursing education environments. Presented at FITNE: Get in touch with multimedia, Atlanta, GA.

Cartwright, G.P. (1994). Information technology: Considerations for tenure and promotion. Change, 6(5), 26-28.

Castner, L. J. (1992). Cognitive strategies of female students using a computer simulation individually and in dyads to identify a nursing diagnosis. (Doctoral dissertation, The Ohio State University, 1992). Dissertation Abstracts International, ATT 9218961 
Charp, S. (2001). Editorial: Assessment and accountability. Technological Horizons in Education (T.H.E.) Journal, 29(9), 7-8.

Cheek, J., Gillham, D., \& Mills, P. (1998, June). Use with care: Possibilities and constraints offered by computers in nursing clinical education. International Journal of Medical Informatics, 50(1-3), 111-115.

Cioffi, J., \& Markhan, R. (1997). Clinical decision-making by midwives: Managing case complexity. Journal of Advanced Nursing, 25(1), 265-72.

Clark, R. (1983). Reconsidering research on learning from media. Review of Educational Research, 53(4), 445-459.

Clark, R. E. (1985a). Confounding in educational computing research. Journal of Educational Computing Research, 1, 28-42.

Clark, R.E. (1985b). Evidence for confounding in computer-based instruction studies: Analyzing meta-analyses. Educational Communications and Technology Journal, 33, 249-262.

Clark, R.E., \& Sugrue, B.M. (1988). Research on instructional media, 1978-1988. Educational media and technology yearbook. Englewood, CO.

Cobb, K. L. (1999). Interactive videodisc instruction with undergraduate nursing students using cooperative learning strategies. Computers in Nursing, 17(2), 89-96.

Cohen, P. A., \& Dacanay, L. S. (1994). A meta-analysis of computer-based instruction in nursing education. Computers in Nursing, 12(2), 89-97.

Collis, B., \& Moonen, J. (2001). Learning in a digital world: Experiences and expectations. Open and distance learning series. Quick Silver Drive, Sterling, VA: Stylus Publishing. 
Conklin, D. N. (1983). A study of computer-assisted instruction in nursing education. Journal of Computer-based Instruction, 9, 98-107.

Cox, A. (1988). The effects of CAI on performing pediatric intravenous medication administration in the clinical setting. Nursing Educators' Microworld, 2(6), 7.

DeAmicis, P.A.(1997). Interactive videodisc instruction is an alternative method for learning and performing a critical nursing skill. Computers in Nursing, 15(3), 155-158.

Dean, R. L. (1989). A Model for Evaluation of the Cost Effectiveness of Computer-Assisted Instruction in a University. Journal of Research on Computing in Education, 21, 277-89.

del Bueno, D. J. (1983). Doing the right thing: Nurses' ability to make clinical decisions. Nurse Educator, 8, 7- 11.

deTornyay, R., \& Thompson, M.A. (1987). Strategies for teaching nursing. New York: John Wiley \& Sons.

Dick, W., \& Carey, L. (1991). The systematic design of instruction. Glenview, IL: Scott Foresman.

Dillman, D.A. (2000). Main and Internet surveys: The tailored design method ( $2^{\text {nd }}$ ed). New York: J. Wiley.

Dimock, P.H., \& Cormier, P. (1991). The effects of format differences and computer experience on performance and anxiety on computer-administered test. Measurement Evaluation Counseling Development, 24, 119-126.

Dupagne, M., \& Krendi, K. (1992). Teachers' attitudes toward computers: A review of the literature. Journal of Computers in Education, 24, 420-429. 
Edwards, M. J. (1995). Using computers in basic nursing education, continuing education and patient education. In M. J. Ball, K. J. Hannah, S. Newbold, J. Douglas (Eds.), Nursing informatics: Where caring and technology meet ( $2^{\text {nd }}$ Ed.). New York: Springer-Verlag

Engberg, S. J., \& White, J. E. (1998). Can computers help us teach clinical decision-making to advanced nursing specialists? Using the computer to teach decision-making. In V. K. Saba; D.B. Pocklington, \& K.P. Miller (Eds.), Nursing and computers: An anthrology, 1987-1996. pp. 574- 580, New York: Springer-Verlag.

Epper, R. L. (2001). The new economy meets the ivory tower. In R. M. Epper \& A.W. Bates (Eds.), Teaching faculty how to use technology: Best practices from learning institutions. Westport, CT: Oryx Press.

Fisher, S. (1997, February 15). The incredible shrinking digital discs are here. Technology for Learning Newsletter. Retrieved June 12, 2000, from http://www.lakewoodpub.com

Flagg, B. N. (1990). Formative evaluation for educational technologies. Hillsdale, NJ: Lawrence, Erlbaum Associates.

Fletcher, J. D. (1992). Cost-effectiveness of interactive courseware. (ERIC Document Reproduction Services No. ED 355 914).

Fletcher-Flynn, C. M., \& Gravatt, B. (1995). The efficacy of computer-assisted instruction (CAI): A meta-analysis. Journal of Educational Computing Research, 12(3), 219-242.

Gaberson, K. B., \& Oermann, M. H. (1999). Clinical teaching strategies in nursing. New York: Springer Series on Teaching of Nursing.

Gagn'e, R. M (1985). The conditions of learning ( $4^{\text {th }}$ ed). New York, NY: Holt, Rinehart and Winston. 
Gagn'e, R. M., Briggs, L., \& Wagner, W. (1992). Principles of instructional design (4 ${ }^{\text {th }}$ ed.). Fort Worth, TX: HBJ College Publishers.

Gaston, S. (1988). Knowledge and attitudes effects of computer-assisted instruction. Journal of Nursing Education, 27, 30-34.

Gay, L. R. (1992). Educational research: Competencies for analysis and application ( $4^{\text {th }}$ ed.). New York: Macmillan Publishing Company.

Gibbons, A. S. \& Fairweather, P.G., O’Neal, A.F. (1993, May). The future of computermanaged instruction. Educational Technology, 33(5), 7-11.

Gibbons, A.S., \& Fairweather, P.G. (1998). Computer-based instruction: Design and development. Englewood Cliffs, NJ: Educational Technology Publications.

Gleydura, A. J., Michelman, J. H., \& Wilson, C. N. (1995). Multimedia training in nursing education. Computers in Nursing, 13(4), 169-174.

Goodman, J., \& Blake, J. A. (1996). Multimedia courseware transforming the classroom. Computers in Nursing, 14(5), 287-296.

Goranson, W. S. (1997). A comparative study on the cost effectiveness of computer-assisted instruction and the traditional lecture. (Doctoral dissertation, Clarkson College, 1997). Dissertation Abstracts International, ATT 1384218

Goyne, J. S., McDonough, S. K., \& Padgett, D.D. (2000). Practical guidelines for evaluating educational software. The Clearing House, 73(6), 345-8.

Green, K. (1996). Campus computing, 1996. The Seventh National Survey of Desktop Computing and Information Technology in Higher Education. Available ERIC Document Reproductive Services ED 405762 
Green, K. (2000). National Survey of Information Technology in Higher Education (Encino, CA: The Campus Computing Project, 2000).

Griepp, M. E. (1988). A plan for implementing computer-assisted instruction. Nurse Educator, $12(2), 17-20$.

Guba, E. G. (1978). Toward a methodology of naturalistic inquiry in educational evaluation. CSE Monograph Series in Education No. 8). Los Angeles, CA: University of California, Center for the Study of Evaluation. (ED 164 599).

Gustafson, K. L. \& Powell, G. C. (1991). Survey of instructional development models with an annotated ERIC Bibliography (2nd ed.). Syracuse, NY: ERIC Clearinghouse on Information Resources. (ED 335 027).

Guyton, J. (1984). The effects of teaching cognitive strategies on problem solving skills of baccalaureate nursing students. Nursing Outlook, 32(1), 54.

Hadley, M., \& Sheingold, K. (1993). Commonalities and distinctive patterns in teachers' integration of computers. American Journal of Education, 101, 261-315.

Hamby, C. S. (1986). A study of the effects of computer-assisted instruction on attitude and achievement of vocational nursing students. Computers in Nursing, 4, 109-113.

Hammond, N., Gardner, N., Heath, S., Kirby, M., Mayes, T., McAleese, R., Mullings, C., \& Trapp, A. (1992). Blocks to the effective use of information technology in higher education. Computers in Education, 18(1-3), 155-162.

Handler, M. (1993). Preparing new teachers to use computer technology: Perceptions and suggestions for teacher educators. Computers in Education, 20, 47-56. 
Hanson, A.C., Foster, S.M., Nassah, B., Hodson, K.E., \& Dillard, N. (1994). Design and development of an expert system for student use in a school of nursing. Computers in Nursing, 12(1), 29-34.

Heinich, R. (1995). The proper study of instructional technology. In G. Anglin (Ed). Instructional technology: Past, present and future ( $2^{\text {nd }}$ ed.). Englewood, CO: Libraries Unlimited, Inc.

Hiltz, S. R. (1990). Collaborative learning: The virtual classroom approach. Technology Horizons in Education Journal (T.H.E.), 17, 59-65.

Hmelo, C. E. (1990). Computer-assisted instruction in health professions education: A review of the published literature. Journal of Educational Technology Systems, 18(2), 8-101.

Huckabay, L. M. D., Anderson, N., Holm, M. H., \& Lee, J. (1979). Cognitive, affective, and transfer of learning consequences of computer-assisted instruction. Nursing Research, $28(4), 228-233$

Ingram, J. K. (1994). A model curriculum to promote teacher-centered use of technology. Peabody Journal of Education, 69(4), 113-30.

Ishida, D., McKnight, P., Solem, S., Tanaka, J., \& Wong, L. (1994). Multi-modal teaching strategies: A “student friendly” approach. Journal of Nursing Education, 33(4), 163-165.

Jairath, N., \& Fitch, M. I. (1994). The generic research protocol: An innovative technique to facilitate research skills development and protocol preparation... a computerized learning package. Journal of Continuing Education in Nursing, 25(3), 111-14.

Jeffries, P. R. (1999). Learning how to perform a 12 lead ECG using virtual reality. Progress in Cardiovascular Nursing, 14(1), 7-13. 
Jenks, J. M. (1993). The pattern of personal knowing in nursing clinical decision-making. Journal of Nursing Education, 32(9), 399-405.

Jenkins, H. (1985). Improving clinical decision-making in nursing. Journal of Nursing Education, 24, 242-243.

Jirapaet, V. (2001). A computer expert system prototype for mechanically ventilated neonates: Development and impact on clinical judgement and information access compatibility of nurses. Computers in Nursing, 19(5), 194-203.

Johnson, D. W., \& Johnson, R. T. (1985). Cooperative learning: One key to computer-assisted learning. The Computing Teacher, 11-15.

Johnstone, D. B., \& Maloney, P.A. (1998). Enhancing productivity of learning: Curricular implications. In J. E. Groccia, \& J. E. Miller (Eds.), Enhancing productivity: Administrative, instructional, and technological strategies (pp. 35-40). San Francisco: Jossey-Bass

Kemp, J. E. \& Dayton, D. K. (1985). Planning and producing instructional media (5 $5^{\text {th }}$ ed). Philadelphia, PA: Harper \& Row.

Kemp, J. E., Morrison, G. R., \& Ross, S. M. (2001). Designing effective instruction (3 ${ }^{\text {rd }}$ ed.), Hoboken, NJ: John Wiley \& Sons, Inc.

Khoiny, F. E. (1995). Factors that contribute to computer-assisted instruction effectiveness. Computers in Nursing, 13(4), 165-8.

Kirchhoff, K. T., \& Holzemer, W.L. (1979). Student learning and a computer-assisted instructional program. Journal of Nursing Education, 18(3), 22-30. 
Kilmon, C. A. (1996). Computerized approaches to teaching nurse practitioner students. Pediatric Nursing, 22(1), 16-8.

Klaassen, E. L. (1988). Exploring the use of computer simulation to teach critical decisionmaking skills to pediatric nursing students. Journal of Pediatric Nursing, 3(3), 202-6.

Koch, E. W., Rankin, J. A., \& Stewart, R. (1990). Nursing students' preferences in the use of computer-assisted learning. Journal of Nursing Education, 3, 122-126.

Kulik, J. A., Bangert, R. L., \& Williams, G. W. (1983). Effects of computer-based teaching on secondary school students. Journal of Educational Psychology, 75, 19-26.

Kulik, J. A. and Kulik, L. C. (1987). Review of recent research literature on computer-based instruction. Contemporary Educational Psychology, 12, 222-230.

Kulik, L. C., \& Kulik, J. A. (1991). Effectiveness of computer-based instruction: An updated analysis. Computers in Human Behavior, 7, 75-94.

Kulik, J. A., \& Kulik, L.C., \& Cohen, P. (1980). Effectiveness of computer-based college teaching: A meta-analysis of findings. Review of Educational Research, 50, 525-544.

Knowles, M. (1984). Andragogy in Action. San Francisco: Jossey-Bass Publishers.

Lange, L., Haak, S., Lincoln, M., Thompson, C., Turner, C., \& Weir, C. (1997). Use of Iliad to improve diagnostic performance of nurse practitioner students. Journal of Nursing Education, 36(1), 36-45.

Larson, D. E. (1982). The use of computer simulation to teach calculation and regulation of intravenous flow rates to nursing students. Paper presented at the Annual Conference of the Association for the Development of Computer Based Instructional Systems, Vancover, British Columbia, Canada, June 1982. 
Laurillard, D. (1993). Rethinking university teaching: A framework for the effective use of educational technology. London: Routledge.

Lerner, H., Cohen, B., \& Brown, M. (1990). Computer usage among high risk Baccalaureate Nursing students. In J. M. Arnold, \& G.A. Pearson (Eds.), Computer applications in nursing education and practice. New York: National League of Nursing Publications.

Levenson, P. M., Morrow, J.R., \& Signer, B. (1985-1986). A comparison of non-interactive and interactive video instruction about smokeless tobacco. Journal of Educational Technology Systems, 14, 193-202.

Levin, H. M. (1983). Cost-effectiveness: A primer. Beverly Hills, CA: Sage Publications.

Levin, H. M. (1986). Costs and cost-effectiveness of computer-assisted instruction: Some insights. Report No. 86-September 13. Sanford, CA: Center for Educational Research at Sanford, School of Education, Stanford University.

Lewis, M. L. (1997). Decision-making task complexity: Model development and initial testing. Journal of Nursing Education, 36(3), 114-120.

Lewis, D. \& Watson, J. E., (1997). Nursing faculty concerns regarding adoption of computer technology. Computers in Nursing, 15(2), 71-76.

Lewis, D., Watson, J. E. \& Newfield, S. (1997). Implementing instructional technology: Strategies for success. Computers in Nursing, 15(4), 187-190.

Lewis, M. J., Davis, R., Jenkins, D. E., \& Tait, M. I. (2001). A review of evaluative studies of computer-based learning in nursing education. Nursing Education Today, 21(1), 26-37. 
Llabre, M., Clements, N., Fitzhugh, K., Lancelotta, G., Mazzagatti, R., Quinones, N. (1987). The effect of computer-administered testing on test anxiety and performance. Journal of Education and Computer Research, 3, 429-433.

Lowdermilk, D.L., \& Fischel, A. (1991). Computer simulations as a measure on nursing student's decision-making skills. Journal of Nursing Education, 30, 34-39.

Lyons, J., \& Milton, J. (1999). Recognizing through feeling: A physical and computer simulation based on educational theory. Computers in Nursing, 17(3), 114-119.

MacKellar, M., \& Elliott, F. (1999). The importance of training CD-ROM end users. Computers in libraries, 19(2), 63-6.

MacKnight, C.B. (1995). Managing technological change in academe. Cause/Effect, 18(1), 2939.

Mallow, G.E., \& Gilje, F. (1999). Technology-based nursing education: Overview and call for further dialogue. Journal of Nursing Education, 38(6), 248-251.

Marcinkiewicz, H. R. (1993). Computers and teachers: Factors influencing computer use in the classroom. Journal of Research on Computing in Education, 26(2), 220-237.

Martin, L. (2001). Knowledge acquisition and evaluation of an expert system for managing disorders of the outer eye. Computers in Nursing, 19(3), 114-117.

Massy, W. F., \& Wilger, A.K. (1998). Technology's contribution to higher education productivity. In J. E., Groccia, \& J. E. Miller (Eds.), Enhancing Productivity: Administrative, instructional, and technological strategies (pp. 49-59). San Francisco: Jossey-Bass. 
Maynard, C.A. (1996). Relationship of critical thinking ability to professional nursing competence. Journal of Nursing Education, 35(1), 12-18.

McAlindon, M. N. (1992). A study to determine the effectiveness of an interactive video instruction program in teaching registered nurses in the clinical setting the process of quality assurance in nursing. (Doctoral dissertation, Wayne State University, Detroit, Michigan, 1992). Dissertation Abstracts International.

McDowell, B. J., Nardini, D. L., Negley, S. A., \& White, J. E. (1984). Evaluating clinical performance using simulated patients. Journal of Nursing Education, 23(1), 37-39.

McFarland, M. (1993). Students' attitudes toward computer-assisted instruction. Nurse Educator, 18(4), 32-33.

McGinty, T., Reed, S., Sautter, R.C. (1987). Software trends and trendsetters: How they are shaping an industry. Electronic Learning, 6(4), 18-24.

Merril, M. D. (1983). Component display theory. In M. Reigeluth (Ed.). Instructional design theories and models. Hillsdale, N J: Lawrence Erlbaum.

Merril, G. L. \& Barker, V. L. (1996). Virtual reality debuts in the teaching laboratory in nursing. Journal of Intravenous Nursing, 19(4), 182-7.

Michelman, J. E. (1994). Have we undervalued the role of technology in accounting education? Journal of Accounting and Computers, 10,1-19.

Mikan, K. \& Aiken, E. (1992). Computer use in undergraduate nursing education programs. In J. M. Arnold, \& G. A. Pearson (Eds.), Computer applications in nursing education and practice. New York: National League of Nursing Publications. 
Miller, E. J. (2000). In videodisc veritas: Interactive video at Harvard Law School. Technological Horizons in Education (T.H.E.) Journal, 17, 78-80.

Neafsey, P.J. (1997). Computer-assisted instruction for home study: A new venture for continuing education programs in nursing. Journal of Continuing Education in Nursing, $28(4), 167-172$.

Niemiec, R., \& Walberg, H. J. (1987). Comparative effects of computer-assisted instruction: A synthesis of reviews. Journal of Educational Computing Research, 3, 19-37.

Noell, J., \& Carnine, D. (1989). Group and individual computer-assisted video instruction. Educational Technology, 29, 36-37.

Oermann, M. H. (1997). Computer use in ADN Programs. Nurse Educator, 22(6), 8.

Office of Technology Assessment (1995). Teachers and technology: Making the connection (OTA-EHR-616). Washington, DC: U.S. Government Printing Office. Retrieved October 20, 2000, from http://www.ota.nap.edu/pdf/1995idx.html

Olsen, J. (1990). Applying computerized adaptive testing in schools. Measurement Evaluation Counseling Development, 23(4), 31-38.

Park, I. H. (1993). Cooperative learning and individual learning with computer-assisted instruction in an introductory university level chemistry course. (Doctoral dissertation, University of Texas at Austin, 1993). Dissertation Abstracts International, AAT 9400967.

Perciful, E. G., \& Nester, P. A. (1996). The effect of an innovative clinical teaching method on nursing students' knowledge and critical thinking skills. Journal of Nursing Education, $35(1), 23-27$. 
Pierson, M. E. (2001). Technology integration practices as a function of pedagogical expertise. Journal of Research on Computing in Education, 33(4), 413-430.

Pond, E. F. (1987). The development and validation of a computer-assisted instructional program in parent-child nursing designed to enhance critical thinking skills of baccalaureate nursing students (Doctoral dissertation, University of Georgia, 1987). Dissertation Abstracts International, AAT 8806824.

Podemski, R.S. (1981). Computer technology and teacher education. Journal of Teacher Education, 32, 29-32.

Pogue, L. M., \& Pogue, R.F. (1998). Integrating computer-assisted instruction into continuing education and inservice training in the practice setting. (1998). In V. K. Saba, D. B. Pocklington, \& K. P. Miller (Eds.), Nursing and computers: An anthrology, 1987-1996. pp. 574- 580, New York: Springer-Verlag.

Publication manual of the American Psychological Association (2001). (5th ed.). Washington, D.C.: American Psychological Association.

Reif, M., Roulis, E., \& Huber, S. (1995). Alternative assessment for adult learners. Retrieved October 20, 2000, from http://www2.nu.edu/nuri/llconf/conf1995/reif.html

Reigeluth, C. M. \& Schwartz, E. (1989). An instructional theory for the design of computer-based simulations. Journal of Computer-Based Instruction, 16(1), 1-10.

Reiser, L.J. (2002). Professional development and other factors that contribute to the ability to integrate technology into curriculum. Journal of Educational Technology System, 30(4), 437-460. 
Reynolds, A. \& Pontius, S. (1986). CAI enhances the medication dosage calculation competency of nursing students. Computers in Nursing, 8, 158-165.

Roblyer, M. D. (1988). The effectiveness of microcomputers in education: A review of the research from 1980-1987. Technological Horizons in Education (T.H.E.) Journal, 8589.

Ronald, J. A. ( 1991). The Computer as a Partner in Nursing Practice: Implications for Curriculum Change. In P. B. Marr, R. L. Axford, R. L. \& S. K. Newbold (Eds.). Nursing Informatics '91: Proceedings of the Post Conference on Health Care Information Technology: Implications for Change. Heidelberg-Berlin, Germany: Springer-Verlag, 149-153.

Rouse, D. P. (1999). Creating an interactive multimedia computer-assisted program. Computers in Nursing, 17(4), 171-176.

Russell, T. (1999). The no significant difference phenomenon. Raleigh, North Carolina State University Office of Instructional Telecommunications.

Sax, L. J., Astin, A.W., Korn, W. S., Gilmartin, S. K. (1999). The American College Teacher: National Norms for the 1998-1999 HERI Faculty Survey. Los Angeles: Higher Education Research Institute, Graduate School of Education and Information Studies, University of California, Los Angeles.

Schaefer, D., \& Dillman, D. (1998). Development of a standardized e-mail methodology: Results of an experiment. Public Opinion Quarterly, 62, 378- 397.

Scanlan, C.L. (1986). Developing computer-assisted learning materials for continuing education in health professionals. Personal computers and the adult learner, 37-46. 
Schare, B. L., Dunn, S. C., Clark, H. M., Soled, S. W., \& Gilman, B. R. (1991). The effects of interactive video on cognitive achievement and attitude toward learning. Journal of Nursing Education, 30, 109-113.

Sittig, D. F., Jiang, S., Manfre, S., Sinkfeld, K., Ginn, R., Smith, L., Olsen, A., \& Borden, R. (1995). Evaluating computer-based experiential learning simulation: A case study using criterion referenced testing. Computers in Nursing, 13, 17-24.

Simpson, J. (1986). Computer and collaborative work among students. Educational Technology, 26, 37-44.

Slavin, R.E. (1983a). Cooperative learning. New York, NY: Longman.

Slavin, R.E. (1983b). When does cooperative learning increase student achievement? Psychological Bulletin, 94, 429-445.

Smith, M.L. \& Glass, G.V. (1987). Research and evaluation in education and the social sciences. Needham Heights, MA: Allyn and Bacon.

Smith, S. G., Jones, L. L., \& Waugh, M. L. (1986). Production and evaluation of interactive videodisc lessons in laboratory instruction. Journal of Computer-Based Instruction, 13, 117-121.

Sparks, S.M., (1989). Computer-based education in nursing (Lister Hill Monograph no. LHNCBC 89-3). Bethesda, MD: United States Department of Health and Human Services, Public Health Service, National Institute of Health, National Library of Medicine. 
Stead, F. L. (1975). An application of the delphi method of forecasting to nursing education planning in West Virginia (Doctoral dissertation, West Virginia University, 1975). Dissertation Abstracts International, AAT 7600765

Straker, N. (1988). Interactive video: A cost-effective model for mathematics and science classrooms. British Journal of Educational Psychology, 19, 202- 210.

Sutter, E. G., Reid, J. B. (1969). Learner variables and interpersonal conditions in computerassisted instruction. Journal of Educational Psychology, 60, 153-157.

Tanner, C.A. (1989). Use of research in clinical judgement. In C. A. Tanner \& C. A. Lindeman, Using research in clinical practice (pp. 19-34). New York: National League for Nursing.

Tanner, C. A. (1993). More thinking about critical thinking and clinical decision-making. Journal of Nursing Education, 32(9), 387.

Thede, L. Q. (1999). Computers in nursing: Bridges to the future. Philadelphia: Lippincott.

Thede. L. Q., Taft, S., \& Coeling, H. (1994). Computer-assisted instruction: A learner's viewpoint. Journal of Nursing Education, 33(7), 299-305.

Theile, J. E., Allen, C., \& Stucky, M. (1999). Effects of web-based instruction on learning behaviors of undergraduate and graduate students. Nursing and Healthcare Perspectives, 20(4), 199-203.

Theile, J. E., Baldwin, J. H., Hyde, R. S., Sloan, B., \& Strandquist, G. A. (1986). An investigation of decision theory: What are the effects of teaching cue recognition? Journal of Nursing Education, 25(8), 319-24. 
Thiele, J. E., \& Holloway, J.R. (1990). Development of a taxonomy of decision-making properties of computerized clinical simulations. In J. M. Arnold, \& G.A. Pearson (Eds.). Computer applications in nursing education and practice. New York: National League of Nursing Publications.

Thurston, C. O., Secaras, E. D., \& Levin, J. A. (1997). Teaching teleapprenticeships: An innovative model for technology integration in teacher education. Journal of Research on Computing in Education, 29(4), 385-91.

Tilson, E. R. (1986). The effect of computer-enhanced skill training in critical thinking and cognitive monitoring on learning and development of critical thinking in an undergraduate family nursing course. (Doctoral dissertation, University of Georgia, 1986). Dissertation Abstracts International, AAT 8706902

Turley, J. (1996). Nursing decision-making and the science of the concrete. Holistic nurse practitioner, 11(1), 6-14.

Umlauf, M.G. (1990). How to provide around-the-clock CPR certification without losing any sleep. The Journal of Continuing Education in Nursing, 21(6), 284-251.

U.S. Congress, Office of Technology Assessment. (1995). Teachers and Technology: Making the Connection, OTA-HER-616. Washington, DC: U.S. Government Printing Office.

Vincent, J. L. (1984). A Comparison of Learning Outcomes of Lecture Discussion and Computer-Assisted Instruction in a Baccalaureate Nursing Program. (Doctoral dissertation, University of Kansas, 1984). Dissertation Abstracts International, AAT 8513794 
Walvoord, B.E. \& Pool, K. J. (1998). Enhancing pedagogical productivity. In J. E., Groccia, \& J. E. Miller (Eds.), Enhancing productivity: Administrative, instructional, and technological strategies (pp. 35- 48). San Francisco: Jossey-Bass.

Warnock-Matheron, A. (1997). Expert systems: Automated decision support for clinical nursing practice. In V. K. Saba, D.B. Pocklington, \& K. P. Miller (Eds.), Nursing and computers: An anthology, 1987-1996. New York: Springer-Verlag.

Watson, G. (1966). Social psychology-issues and insights. Philadelphia, PA: Lippincott.

Waxman, H.C., \& Huang, S.Y. (1996). Classroom instruction differences by level of technology use in middle school mathematics. Journal of Educational Computing Research, 14(2), 157-169.

Webb, N.M. (1985). Cognitive requirements for learning computer programming in group and individual settings. AEDS Journal, 18, 183-194.

Weiss, P.A. \& Guyton-Simmons, J. (1998). A computer simulation for teaching critical thinking skills. Nurse Educator, 23(2), 30-33.

Weiss, C., \& Jarvis, S. (1986-87). The interactive videodisc for computer-assisted education and training in developing countries. Journal of Educational Technology Systems, 15, 3-18. Wells, F.S. \& Kick, R.C. (1996). Enhancing teaching and learning in higher education with a total multimedia approach. Proceedings of the Mid-South Instructional Technology Conferences ( $1^{\text {st }}$, Murfreesboro, Tennessee, March 31- April 2, 1996).

Whipple, W.R. (1987). Collaborative learning: Recognizing it when we see it. Bulletin of the American Association for Higher Education, 40, 3-7. 
White, J. E. (1995). Using interactive video to add physical assessment data to computer-based simulations in nursing. Computers in nursing, 13(5), 233-235.

Whyte, M.E. (1990). Individualistic versus paired/cooperative computer-assisted instruction: Matching instructional method with cognitive style. (Doctoral Dissertation, University of Southern California, 1990). Copies available: Micrographics Department, Doheny Library, USC, Los Angeles, CA 90089-0182.

Willis, B. (1993). Distance education: A practical guide. Englewood Cliffs, NJ: Educational Technology Publications.

Wilson, F. R., Genco, K. T., \& Yager, G. C. (1985). Assessing the demonstration of a promising methodology. Computer and Human Behavior, 1, 265-275.

Wittrock, M.C. (1991). Generative teaching of comprehension. Elementary School Journal, 92(2), 169-184.

Wise, S.L., \& Plake, B.S. (1990). Computer-based testing in higher education. Measurement Evaluation Counseling Development, 23(4), 3-10.

Wizer, D.R. (1987). Cooperative learning with microcomputers. The Pointer, 32, 31-33.

Worthen, B.R., Van Dusen, L.M., \& Sailor, P.J. (1994). A comparative study of the impact of integrated learning systems on student's time-on-task. International Journal of Educational Research, 21(1), 25-37.

Yanok, J. 1988). Individualized instruction: A good approach. Academic Therapy, 24(2), $163-$ 167.

Young, J. R. (1997). Invasion of the laptops: More colleges adopt mandatory computing programs. The Chronicle of Higher Education, 44 (15), A33-A35. 
Appendix A

Instruments and Correspondence with Participants

Integration of CAI Questionnaire (Electronic Version)

\section{Technology Education West Virginia University \\ Department of Advanced Educational Studies}

\section{Consent and Information Form}

\section{Complete title of the research report:}

The Effective Integration of Computer-assisted Instruction to Teach Clinical Decision-making

\section{Introduction}

You are being asked to participate in this research study being conducted by Beverly J. Schaefer R.N., M.S.N. under the supervision of David McCrory Ph.D. The research is being conducted to fulfill the requirements for a doctoral dissertation in Technology Education in the Department of Advanced Educational Studies at West Virginia University.

\section{Purposes of the Study}

The purposes of this study are to learn more about the integration strategies nurse educators in the United States use to integrate computer-assisted instruction into the courses they teach. It will also provide information about the types of software programs nurse educators use to teach students clinical decision-making skills.

\section{Description of Procedures}

The study involves completing an online questionnaire by clicking with your mouse on a radio button or typing in an answer to indicate the answer that best describes the teaching strategies you and your colleagues use. It will take approximately twenty to thirty minutes to complete. Approximately 140 subjects are expected to participate in this study. If you decide to participate, you do not have to answer all the questions.

\section{Risks and Discomforts}

There are no known risks and no discomfort is expected from participation in this study.

\section{Alternatives}

You do not have to participate in this study.

\section{Benefits}

Your participation in this study will not be of direct benefit you, but the knowledge gained may be of benefits to others. 


\section{Contact Persons}

For more information about this research, please contact Beverly J. Schaefer at BeverlySchaefer@cs.com or by phone at 440/350-7229 or her academic advisor, Dr. David McCrory at 304/293-3803 Ext. 1706. For information regarding your rights as a research subject, you may contact the Executive Secretary of the Institutional Review Board at 304/293-7073.

\section{Confidentiality}

Any information about you obtained as a result of your participation in this research will be kept as confidential as legally possible. Research records and test results, just like hospital records, may be subpoenaed by court order. In any publications that may result from this research, neither your name nor any information from which you might be identified will be published without your consent.

\section{Voluntary Participation}

Participation in this study is voluntary. You are free to withdraw your consent to participate in this study at any time and such refusal will not affect your employment status or your employer in any way. Refusal to participate or your decision to withdraw will involve no penalty to you. Please feel free to ask any questions you may have about the research before you participate. In the event new information becomes available that may affect your willingness to continue to participate in this study, this information will be given to you so that you may make an informed decision about your participation.

If you would like to continue and you are willing to participate in this study, click on this button.

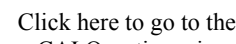

Beverly J. Schaefer R.N., M.S.N.

Signature of the Investigator

September 12, 2003

Date

IRB Approval Stamp is on file at the office of the primary investigator at 10242 Lola Court, Concord, $\mathrm{OH}$ 44077. 
Directions: Please click on the box to indicate your answer and click on underlined words to view the definition.

1. Have you used computer-assisted instruction (CAI) to teach clinical decision-making over the past year?

Yes - (If no, click to skip to question 11)

2. How OFTEN did you use each of the following strategies to integrate CAI into the courses you taught during the past year?

\begin{tabular}{|c|c|c|c|c|c|}
\hline HOW OFTEN STRATEGIES WERE USED? & $\underset{y}{\text { Weekl }}$ & Monthly & Semester & Yearly & Never \\
\hline \multicolumn{6}{|l|}{$\begin{array}{l}\text { A. Pre-test learners to be sure they are adequately prepared to learn the } \\
\text { content of the CAI program }\end{array}$} \\
\hline \multicolumn{6}{|l|}{ B. Explain the objectives of the CAI program to students beforehand } \\
\hline \multicolumn{6}{|l|}{ C. Explain how to use the CAI program to students beforehand } \\
\hline \multicolumn{6}{|l|}{$\begin{array}{l}\text { D. Motivate students by explaining the value of learning the content of the } \\
\text { CAI }\end{array}$} \\
\hline \multicolumn{6}{|l|}{$\begin{array}{l}\text { E. Illustrate relationships between topics in order to integrate the topic of } \\
\text { the CAI with the rest of the course }\end{array}$} \\
\hline \multicolumn{6}{|l|}{$\begin{array}{l}\text { F. Assign CAI programs as self-paced, individualized learning } \\
\text { experiences }\end{array}$} \\
\hline \multicolumn{6}{|l|}{ G. Use CAI programs for small-group learning activities } \\
\hline \multicolumn{6}{|l|}{ H. Use CAI in the classroom as part of a whole-class learning activity } \\
\hline \multicolumn{6}{|l|}{ I. Ensure technical support is available for students who need it } \\
\hline \multicolumn{6}{|l|}{ J. Ensure instructional support is available for students who need it } \\
\hline \multicolumn{6}{|l|}{$\begin{array}{l}\text { K. Conduct post-instruction discussions or critiques in order to reinforce } \\
\text { what students learned and correct misunderstandings }\end{array}$} \\
\hline \multicolumn{6}{|l|}{$\begin{array}{l}\text { L. Use follow-up activities to ensure students use what they learned in the } \\
\text { CAI program }\end{array}$} \\
\hline \multicolumn{6}{|l|}{ M. Make completion of the CAI program part of the course requirements } \\
\hline \multicolumn{6}{|l|}{ N. Assign the CAI program as an optional learning experience } \\
\hline \multicolumn{6}{|l|}{$\begin{array}{l}\text { O. Include the content or skills learned from the CAI program on the } \\
\text { course exams }\end{array}$} \\
\hline \multicolumn{6}{|l|}{$\begin{array}{l}\text { P. Include scores earned on tests embedded in the CAI as part of the } \\
\text { course grade }\end{array}$} \\
\hline \multicolumn{6}{|l|}{$\begin{array}{l}\text { Q. Use the CAI program in place of some other method of teaching the } \\
\text { content }\end{array}$} \\
\hline \multicolumn{6}{|l|}{$\begin{array}{l}\text { R. Provide alternative methods of restudying if results of tests indicate } \\
\text { students failed to meet objectives }\end{array}$} \\
\hline $\begin{array}{l}\text { S. Conduct summative evaluations of student learning to test the } \\
\text { effectiveness of the CAI in meeting course objectives }\end{array}$ & & & & & \\
\hline
\end{tabular}


3. Based on your experience, do you agree or disagree that the following integration strategies are effective for increasing STUDENT LEARNING? Please make your selection based on what you've observed when the particular strategy was either used or not used by a teacher.

\begin{tabular}{|c|c|c|c|c|}
\hline STUDENT LEARNING & $\begin{array}{l}\text { Strongly } \\
\text { Agree }\end{array}$ & Agree & Disagree & $\begin{array}{l}\text { Strongly } \\
\text { Disagree }\end{array}$ \\
\hline \multicolumn{5}{|l|}{$\begin{array}{l}\text { A. Pre-test learners to be sure they are adequately prepared to learn the content of the } \\
\text { CAI program }\end{array}$} \\
\hline \multicolumn{5}{|l|}{ B. Explain the objectives of the CAI program to students beforehand } \\
\hline \multicolumn{5}{|l|}{ C. Explain how to use the CAI program to students beforehand } \\
\hline \multicolumn{5}{|l|}{ D. Motivate students by explaining the value of learning the content of the CAI } \\
\hline \multicolumn{5}{|l|}{$\begin{array}{l}\text { E. Illustrate relationships between topics in order to integrate the topic of the CAI } \\
\text { with the rest of the course }\end{array}$} \\
\hline \multicolumn{5}{|l|}{ F. Assign CAI programs as self-paced, individualized learning experiences } \\
\hline \multicolumn{5}{|l|}{ G. Use CAI programs for small-group learning activities } \\
\hline \multicolumn{5}{|l|}{ H. Use CAI in the classroom as part of a whole-class learning activity } \\
\hline \multicolumn{5}{|l|}{ I. Ensure technical support is available for students who need it } \\
\hline \multicolumn{5}{|l|}{ J. Ensure instructional support is available for students who need it } \\
\hline \multicolumn{5}{|l|}{$\begin{array}{l}\text { K. Conduct post-instruction discussions or critiques in order to reinforce what } \\
\text { students learned and correct misunderstandings }\end{array}$} \\
\hline \multicolumn{5}{|l|}{$\begin{array}{l}\text { L. Use follow-up activities to ensure students use what they learned in the CAI } \\
\text { program }\end{array}$} \\
\hline \multicolumn{5}{|l|}{ M. Make completion of the CAI program part of the course requirements } \\
\hline \multicolumn{5}{|l|}{ N. Assign the CAI program as an optional learning experience } \\
\hline \multicolumn{5}{|l|}{ O. Include the content or skills learned from the CAI program on the course exams } \\
\hline \multicolumn{5}{|l|}{ P. Include scores earned on tests embedded in the CAI as part of the course grade } \\
\hline \multicolumn{5}{|l|}{ Q. Use the CAI program in place of some other method of teaching that content } \\
\hline \multicolumn{5}{|l|}{$\begin{array}{l}\text { R. Provide methods of restudying if results of tests indicate students failed to meet } \\
\text { objectives }\end{array}$} \\
\hline $\begin{array}{l}\text { S. Conduct summative evaluations of student learning to test the effectiveness of the } \\
\text { CAI in meeting course objectives }\end{array}$ & & & & \\
\hline
\end{tabular}


4. Based on your experience, do you agree or disagree that the following integration strategies are effective for REDUCING THE COSTS OF THE COURSE? Please make your selection based on what you've observed when the particular strategy was either used or not used by a teacher.

COSTS OF THE COURSE

F. Assign CAI programs as self-paced, individualized learning experiences

G. Use CAI programs for small-group learning activities

H. Use CAI in the classroom as part of a whole-class learning activity

I. Ensure technical support is available for students who need it

J. Ensure instructional support is available for students who need it

M. Make completion of the CAI program part of the course requirements

N. Assign the CAI program as an optional learning experience

Q. Use the CAI program in place of some other method of teaching that content

5. Based on your experience, do you agree or disagree that the following integration strategies are effective for REDUCING THE TIME STUDENTS SPEND on the learning task? Please make your selection based on what you've observed when the particular strategy was either used or not used by a teacher.

\begin{tabular}{|c|c|c|c|c|}
\hline REDUCE STUDENT TIME ON TASK & $\begin{array}{l}\text { Strongly } \\
\text { Agree }\end{array}$ & Agree & Disagree & $\begin{array}{l}\text { Strongly } \\
\text { Disagree }\end{array}$ \\
\hline \multicolumn{5}{|l|}{ B. Explain the objectives of the CAI program to students beforehand } \\
\hline \multicolumn{5}{|l|}{ C. Explain how to use the CAI program to students beforehand } \\
\hline \multicolumn{5}{|l|}{ F. Assign CAI programs as self-paced, individualized learning experiences } \\
\hline \multicolumn{5}{|l|}{ G. Use CAI programs for small-group learning activities } \\
\hline \multicolumn{5}{|l|}{ H. Use CAI in the classroom as part of a whole-class learning activity } \\
\hline \multicolumn{5}{|l|}{ I. Ensure technical support is available for students who need it } \\
\hline J. Ensure instructional support is available for students who need it & & & & \\
\hline
\end{tabular}


6. Based on your experience, how strongly do you agree or disagree that the following integration strategies are effective for REDUCING THE TIME TEACHERS SPEND on the learning task? Please make your selection based on what you've observed when the particular strategy was either used or not used by a teacher.

\begin{tabular}{|c|c|c|c|c|}
\hline TEACHER'S TIME ON TASK & $\begin{array}{l}\text { Strongly } \\
\text { Agree }\end{array}$ & Agree & Disagree & $\begin{array}{l}\text { Strongly } \\
\text { Disagree }\end{array}$ \\
\hline \multicolumn{5}{|l|}{ F. Assign CAI programs as self-paced, individualized learning experiences } \\
\hline \multicolumn{5}{|l|}{ G. Use CAI programs for small-group learning activities } \\
\hline \multicolumn{5}{|l|}{ H. Use CAI in the classroom as part of a whole-class learning activity } \\
\hline \multicolumn{5}{|l|}{ I. Ensure technical support is available for students who need it } \\
\hline \multicolumn{5}{|l|}{ J. Ensure instructional support is available for students who need it } \\
\hline \multicolumn{5}{|l|}{ M. Make completion of the CAI program part of the course requirements } \\
\hline \multicolumn{5}{|l|}{ N. Assign the CAI program as an optional learning experience } \\
\hline \multicolumn{5}{|l|}{ O. Include the content, or skills learned from the CAI program on the course exams } \\
\hline \multicolumn{5}{|l|}{ P. Include scores earned on tests embedded in the CAI as part of the course grade } \\
\hline Q. Use the CAI program in place of some other method of teaching that content & & & & \\
\hline
\end{tabular}

7. Over the past year, how many of each type of CAI software program have the teachers are your school of nursing used to teach students clinical decision-making? Definitions for each type of software are on the last page.

(0- 999)

\begin{tabular}{|l|l|}
\hline Simulations & \\
\hline Tutorial & \\
\hline Virtual reality & \\
\hline Expert Systems (AI) & \\
\hline Drill-and-Practice & \\
\hline
\end{tabular}


8. How strongly do you agree or disagree with the statement, "teachers at your school of nursing were able to effectively integrate each of the following types of software designed to teach clinical decision-making into the courses they taught?" Please make your selection based on what you've observed when the various types of software were used.

\begin{tabular}{|l|c|l|l|l|}
\hline & $\begin{array}{c}\text { Strongly } \\
\text { Agree }\end{array}$ & Agree & Disagree & $\begin{array}{c}\text { Strongly } \\
\text { Disagree }\end{array}$ \\
\hline Simulations & & & & \\
\hline Tutorials & & & & \\
\hline Virtual Reality & & & & \\
\hline Expert Systems (Artificial Intelligence) & & & & \\
\hline Drill and Practice & & & & \\
\hline
\end{tabular}

9. Of the CAI programs used at your school over the past year to teach clinical decision-making, how many were:

$(0-999)$

\begin{tabular}{|l|l|}
\hline Commercially produced programs - purchased through vendors, book publishers, etc. & \\
\hline Customized programs - produced in-house by a multi-disciplinary team & \\
\hline Teacher-authored programs- one or two authors from your school & \\
\hline
\end{tabular}

10. How strongly do you agree or disagree with the following statement: "teachers at your school of nursing were able to effectively integrate the following types of software designed to teach clinical decision-making into the courses they taught?" Please make your selection based on what you've observed when the various types of software were used.

\begin{tabular}{|l|l|l|l|l|}
\hline \multicolumn{1}{|c|}{ (Place an "X"next to the correct response) } \\
\hline & $\begin{array}{c}\text { Strongly } \\
\text { Agree }\end{array}$ & Agree & Disagree & $\begin{array}{c}\text { Strongly } \\
\text { Disagree }\end{array}$ \\
\hline Commercially purchased programs & & & & \\
\hline $\begin{array}{l}\text { Customized programs produced at the school } \\
\begin{array}{l}\text { Teacher-authored programs - one or two authors from } \\
\text { your school }\end{array}\end{array}$ & & & & \\
\hline
\end{tabular}

And now, please take an additional moment to provide some information about yourself and your school.

11. What type of institution is your school? (Place an "X")

\begin{tabular}{|l|l|}
\hline Public & \\
\hline Private & \\
\hline
\end{tabular}


12. What type of program is the school of nursing? (Place an "X")

\begin{tabular}{|l|l|}
\hline $\begin{array}{l}\text { Bachelors } \\
\text { Degree }\end{array}$ & \\
\hline Associate Degree & \\
\hline Diploma & \\
\hline
\end{tabular}

13. How many students are enrolled in the school of nursing? (1- 999)

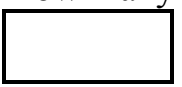

14. Does the school of nursing have its own computer laboratory? (Place an "X")

\begin{tabular}{|l|l|}
\hline Yes & \\
\hline No & \\
\hline
\end{tabular}

15. Where are the computers students use located? (Place an "X" next to all that apply)

\begin{tabular}{|l|l|}
\hline School of nursing & \\
\hline Another department & \\
\hline Library & \\
\hline Students purchase their own & \\
\hline
\end{tabular}

16. How many computers are available for students to use at each location? (0-999)

\begin{tabular}{|l|l|}
\hline School of Nursing & \\
\hline Another department & \\
\hline Library & \\
\hline
\end{tabular}

17. How many support people work in the computer lab? (0-999)

\begin{tabular}{|l|l|l|}
\hline Number of Employees & Part Time & Full Time \\
\hline Technical support & & \\
\hline Instructional support & & \\
\hline
\end{tabular}

18. How many and what percent of the faculty at your school of nursing use CAI to teach clinical decision-making?

\begin{tabular}{|l|l|}
\hline Number of teachers who use CAI? $\quad(0-999)$ & \\
\hline What percent of the faculty is that number? $(0-100 \%)$ & \\
\hline
\end{tabular}


19. What is your role at the school? (Place an X next to all that apply)

\begin{tabular}{|l|l|}
\hline Faculty School of Nursing & \\
\hline Faculty another department & \\
\hline Computer Lab employee & \\
\hline Other (please specify) & \\
\hline
\end{tabular}

20. Are you: (Place an "X”)

\begin{tabular}{|l|l|}
\hline Male & \\
\hline Female & \\
\hline
\end{tabular}

21. Your age range: (Place an " $\mathrm{X}$ ")

\begin{tabular}{|l|l|}
\hline $20-30$ years & \\
\hline $31-40$ years & \\
\hline $41-50$ years & \\
\hline $51-60$ or over & \\
\hline
\end{tabular}

22. Your educational background (Place an " $\mathrm{X}$ " beside all that apply)

\begin{tabular}{|l|l|}
\hline BSN & \\
\hline MSN & \\
\hline Ph.D. Nursing & \\
\hline $\begin{array}{l}\text { Other (please } \\
\text { specify) }\end{array}$ & \\
\hline
\end{tabular}

23. Please write in any other comments you would like to offer that might shed light on this study or your responses.

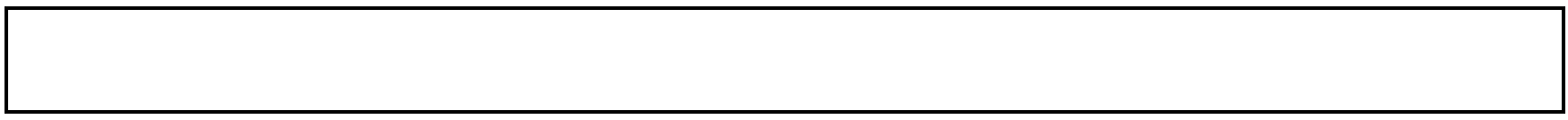

Please enter the authorization code you were provided with the invitation to participate in this study.

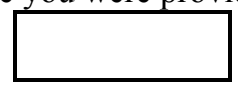

Thank you once again for your participation. 


\section{Definition of Terms}

In this study, COMPUTER-ASSISTED INSTRUCTION (CAI) refers to software programs that employ various strategies and techniques to teach or provide students with practice learning cognitive skills such as decision-making. Instructional software may be purchased commercially on a CD-ROM, interactive video disc (IVD), DVD, a 3.5 disc or

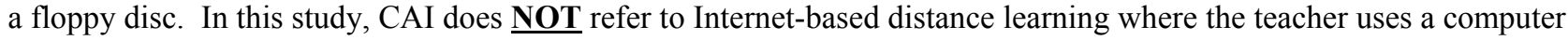
to guide learning or to conduct electronic discussions with students.

CLINICAL DECISION-MAKING is the operationalization of nursing knowledge. It is a complex series of decisions nurses make about what to observe, how to intervene, and then, based on evaluation of the patient's response, what further actions should be taken. Clinical decisions are made by nurses with and on behalf of patients for the purpose of attaining the patient's desired goals.

INTEGRATION STRATEGIES entail the skillful planning and management of instruction by the teacher in order to increase the productivity and effectiveness of the course. The focus of integration strategies are to optimize student learning, reduce the amount of time students and teachers spend on the task and reduce course costs in order to gain the most from the large financial investment that accompanies the use of CAI.

SIMULATION software programs are abstractions or simplified representations of a system, a situation, or set of processes. Students take on a prescribed role and interact with other participants or elements of the simulated environment. There are basically two types of simulations; experiential and symbolic. In an experiential simulation, the student is placed in the situation and reacts to situations as they emerge. In a symbolic simulation, the student remains "outside" the simulated environment but exerts control by adding, removing, altering or selecting correct variables.

TUTORIAL programs present a lesson in basically the same way a teacher in a classroom presents new information. Lessons usually begin by presenting facts and then progress to explaining principles or illustrating a procedure. Many tutorial programs contain a presentation and a practice portion in which students are tested for mastery of the content before proceeding to more difficult lessons.

VIRTUAL REALITY (VR) software programs combine computer software and hardware (i.e., a head mounted visual display helmet, special gloves, or a mannequin) to create a computer-generated environment that is in someway life-like. Desktop VR simulators integrate clinical decision-making with learning a procedure such as administering cardiopulmonary resuscitation, examining the abdomen of a pregnant woman or inserting an intravenous catheter. Learners are able to visualize, manipulate, and interact with complex objects that are portrayed on a computer display screen as they practice their decision-making and problem-solving skills. Some types of VR software completely immerse the user in the environment and provide a sense of immediacy and control created by the immersion: the feeling of being there or presence that comes from a changing visual display that is dependent upon eye or hand or instrument movements.

EXPERT SYSTEMS software is an outgrowth of artificial intelligence in which the computer duplicates the decisionmaking of a human expert in a particular profession. The software is used to assist and train professionals and novices in decision-making tasks. Development of the software is accomplished by capturing and incorporating the knowledge, experience and rules-of-thumb employed by human experts. Depending on the sophistication of the program, when they are queried, some systems give a diagnosis, call for general and specific knowledge and employ tricks-of-the-trade, include exceptions, reasonable guesses and are able to reason from partial knowledge.

DRILL-AND-PRACTICE software is appropriate for educational instruction in higher education when rote memorization or practice in basic skills such as drug calculations is the goal. The software provides a reliable means for assessing mastery level and reduces the time required for learning by providing students with immediate feedback on their progress. Drill-and-practice software is seldom used to teach higher level cognitive skills, such as decision making, but data bases of higher level multiple-choice questions are available to provide students with practice in test-taking skills that resemble state board examination questions. 


\section{A 2. Initial Email Contact Note}

\section{Technology Education West Virginia University College of Human Resources and Education}

Dear [name field],

I am conducting a dissertation research study to determine how nurse educators from across the United States integrate computer-assisted instruction software programs into the courses they teach. Your school was randomly selected from a list of almost 1400 schools to participate. The purpose of this email is to ask for your assistance in identifying a faculty member from your school who would be interested in participating in the study. It would require approximately 20 minutes time and would entail completing an online questionnaire that asks about common practices at your school. The qualifications for participants are as follows:

1) that they be a member of the faculty of your [type of school field] school of nursing,

2) someone who often works with other faculty and students who are using computer-assisted instruction (simulation, tutorial, virtual reality, or expert systems software) to learn clinical decisionmaking skills, and

3) that they be willing to participate.

I would appreciate it if you could forward this message to the person who meets the qualifications that are listed above or send an email address to me where they can be reached so I can contact them. Once the message has been forwarded, I would ask that they simply respond to it by sending me an email address where I can send the cover letter that explains the study more fully and the URL link to the CAI Questionnaire.

PLEASE NOTE: Even if your school does not use computer-assisted instruction to teach students clinical decision-making skills, your participation would still provide valuable information in that it would help me to better understand what is happening in the "real world" of nursing education.

Thank you so much for your assistance.

Sincerely, Beverly J. Schaefer R.N., M.S.N..

Primary Investigator $\backslash$ Doctoral Candidate

West Virginia University

Department of Advanced Educational Studies

334 293-3803 509 Allen Hall P.O. Box 6122 Morgantown, WV 26506-6122

Equal Opportunity / Affirmative Action Institution

A 3. Cover Email Letter 


\section{Technology Education \\ West Virginia University \\ College of Human Resources and Education}

Dear Participant,

May I begin by thanking you for the interest you've expressed in participating in this important research study. To clarify, this is my dissertation research study that is being conducted in partial fulfillment of the requirements I must meet in order to complete a Doctorate in Technology Education. The purpose of the study is to identify the integration strategies nurse educators in the United States use to integrate computer-assisted instruction (CAI) software that is designed to teach clinical decisionmaking into their courses. Let me repeat that again in different words just to be sure you understand; the focus is on teaching and what nursing educators do and not on how well the computer-assisted instruction does what it does. What I discovered from the literature was that there are four basic reasons nursing teachers use CAI. They are (1) to increase student learning, (2) to reduce course costs, (3) to reduce the time students spend on the learning task, or (4) to reduce the time the teacher spends teaching. There are various strategies teachers use to integrate the CAI learning experience into the course and they are the primary focus of this study. In the end, I hope to be able to tell you and other nurse educators which strategies nurse educators are using and how effective a randomly selected group of educators perceive each one is for increasing student learning, reducing course costs, students' time-on-task and teachers' time along with many other facts about how CAI is being used.

If your school does not use CAI or uses it very little, your participation is just as valuable as those who use it often because it will provide me with a balanced and accurate picture of what nurse educators are actually doing. Please just answer the questions as honestly as you possibly can.

May I take this opportunity to say thank you once again for your time and effort. It is greatly appreciated. If you have any questions, please feel free to contact me at BeverlySchaefer@cs.com or by phone at (440) 350-7229. If it is a toll call, please feel free to call and give me your number and I'll call you right back. I have unlimited long distance calling. It should take you approximately 20 to 30 minutes to complete the on-line questionnaire.

Your access code is [individual's code number]. Please write this number down because you will need it at the end in order to submit your questionnaire. The URL address to the questionnaire is at http://www.dalesplace.net/survey/CAIQuestionnaire.htm

Sincerely,

Beverly J. Schaefer R.N., M.S.N..

Primary Investigator

West Virginia University

334 293-3803 509 Allen Hall P.O. Box 6122 Morgantown, WV 26506-6122

Equal Opportunity / Affirmative Action Institution 


\section{A 4. Reminder Email Note}

\section{Technology Education}

West Virginia University

College of Human Resources and Education

Dear Participant,

This is a reminder message intended to jog your memory about the nursing education research study on the use of CAI you agreed to complete. I recognize the winter break may have distracted you from completing the study when I sent it out in mid-December but I'm hoping that you will be able to take a few minutes now to answer the questions. If, for some reason, you won't be able to take part in the study please write to me at: BeverlySchaefer@cs.com to let me know.

To refresh your memory, the purpose of the study is to identify the integration strategies nurse educators in the United States use to integrate computer-assisted instruction (CAI) software designed to teach clinical decision-making into their courses. Again, the focus is on teaching and what nursing educators do and not on how well the computer-assisted instruction does what it does. What I discovered from the literature was that the four basic reasons nursing teachers use CAI are: (1) to increase student learning, (2) to reduce course costs, (3) to reduce the time students spend on the learning task, or (4) to reduce the time the teacher spends teaching. All are valid reasons and there is evidence that CAI may promote all four. There are also many different teaching strategies teachers use to integrate the CAI learning experience into the courses they teach and THEY are the primary focus of this study. In the end, I hope to be able to tell you and other nurse educators which strategies nurse educators are using and how effective a randomly selected group of educators PERCEIVE each one is for increasing student learning, reducing course costs, students' time-on-task and teachers' time along with many other facts about how CAI is being used.

Please Note: if your school does not use CAI or uses it very little, your participation is just as valuable as those who use it frequently because your feedback will provide me with an accurate picture of what nurse educators are actually doing. Just answer the questions as honestly as you can and if they don't pertain to your school skip the first section and answer the questions in the second half. You do not need to answer every question.

May I take this opportunity to say thank you once again for your time and effort. It is greatly appreciated. If you have any questions, please feel free to contact me at BeverlySchaefer@cs.com or by phone at (440) 350-7229. If it is a toll call, please feel free to call and give me your number and I'll call you right back. I have unlimited long distance calling. It should take you approximately 20 to 30 minutes to complete the on-line questionnaire.

Your access code is [CODE]. Please write this number down because you will need it at the end in order to submit your questionnaire. The URL address to the CAI questionnaire is at http://www.dalesplace.net/survey/CAIQuestionnaire.htm

Sincerely,

Beverly J. Schaefer R.N., M.S.N..

Primary Investigator

West Virginia University 
Appendix B

Non-response Bias-check Findings

Table B 1. CAI-user and Non-User Schools: Non-Response Bias-check

\begin{tabular}{|c|c|c|}
\hline & Sample & Non-respondents \\
\hline \multirow{3}{*}{ CAI-users } & 77 & 7 \\
\hline & $71 \%$ & $100 \%$ \\
\hline & 32 & 0 \\
\hline \multirow[t]{2}{*}{ Non-users } & $29 \%$ & $0 \%$ \\
\hline & 109 & 7 \\
\hline Total & $100 \%$ & $100 \%$ \\
\hline
\end{tabular}


Table B 2. Types of Schools and Institutions in Sample and Non-respondent Group: NonResponse Bias-check

Sample Schools:

\begin{tabular}{|c|c|c|c|c|c|c|}
\hline \multicolumn{3}{|c|}{ Public } & \multicolumn{2}{|c|}{ Private } & \multicolumn{2}{|c|}{ Total } \\
\hline \multirow{3}{*}{ Bachelors } & 30 & & 15 & & 45 & \\
\hline & & $36 \%$ & & $58 \%$ & & $41 \%$ \\
\hline & 53 & & 6 & & 59 & \\
\hline \multirow[t]{2}{*}{ Associate } & & $64 \%$ & & $23 \%$ & & $54 \%$ \\
\hline & 0 & & 5 & & 5 & \\
\hline \multirow[t]{2}{*}{ Diploma } & & $0 \%$ & & $19 \%$ & & $5 \%$ \\
\hline & 83 & & 26 & & 109 & \\
\hline Total & & $100 \%$ & & $100 \%$ & & $100 \%$ \\
\hline
\end{tabular}

Non-respondent Schools:

\begin{tabular}{|c|c|c|c|c|c|c|}
\hline \multicolumn{3}{|c|}{ Public } & & \multicolumn{3}{|c|}{ Total } \\
\hline \multirow{3}{*}{ Bachelors } & 2 & & 2 & & 4 & \\
\hline & & $40 \%$ & & $100 \%$ & & $57 \%$ \\
\hline & 3 & & 0 & & 3 & \\
\hline \multirow[t]{2}{*}{ Associate } & & $60 \%$ & & $0 \%$ & & $43 \%$ \\
\hline & 0 & & 0 & & 0 & \\
\hline \multirow[t]{2}{*}{ Diploma } & & $0 \%$ & & $0 \%$ & & $0 \%$ \\
\hline & 5 & & 2 & & 1 & \\
\hline Total & & $100 \%$ & & $100 \%$ & & $100 \%$ \\
\hline
\end{tabular}


Table B 3. Average Student Enrollments in Sample and Non-respondent Groups: Bias-check

Sample Schools

\begin{tabular}{|c|c|c|c|}
\hline & Public & Private & Total \\
\hline \multirow{3}{*}{ Bachelors } & 256 & 233 & 489 \\
\hline & $46 \%$ & $39 \%$ & $42 \%$ \\
\hline & 205 & 202 & 407 \\
\hline \multirow[t]{2}{*}{ Associate } & $37 \%$ & $34 \%$ & $35 \%$ \\
\hline & 100 & 155 & 255 \\
\hline \multirow[t]{2}{*}{ Diploma } & $18 \%$ & $26 \%$ & $22 \%$ \\
\hline & 561 & 590 & 1,151 \\
\hline Total & $100 \%$ & $100 \%$ & $100 \%$ \\
\hline Percent & $49 \%$ & $51 \%$ & $100 \%$ \\
\hline
\end{tabular}

Non-respondent Schools:

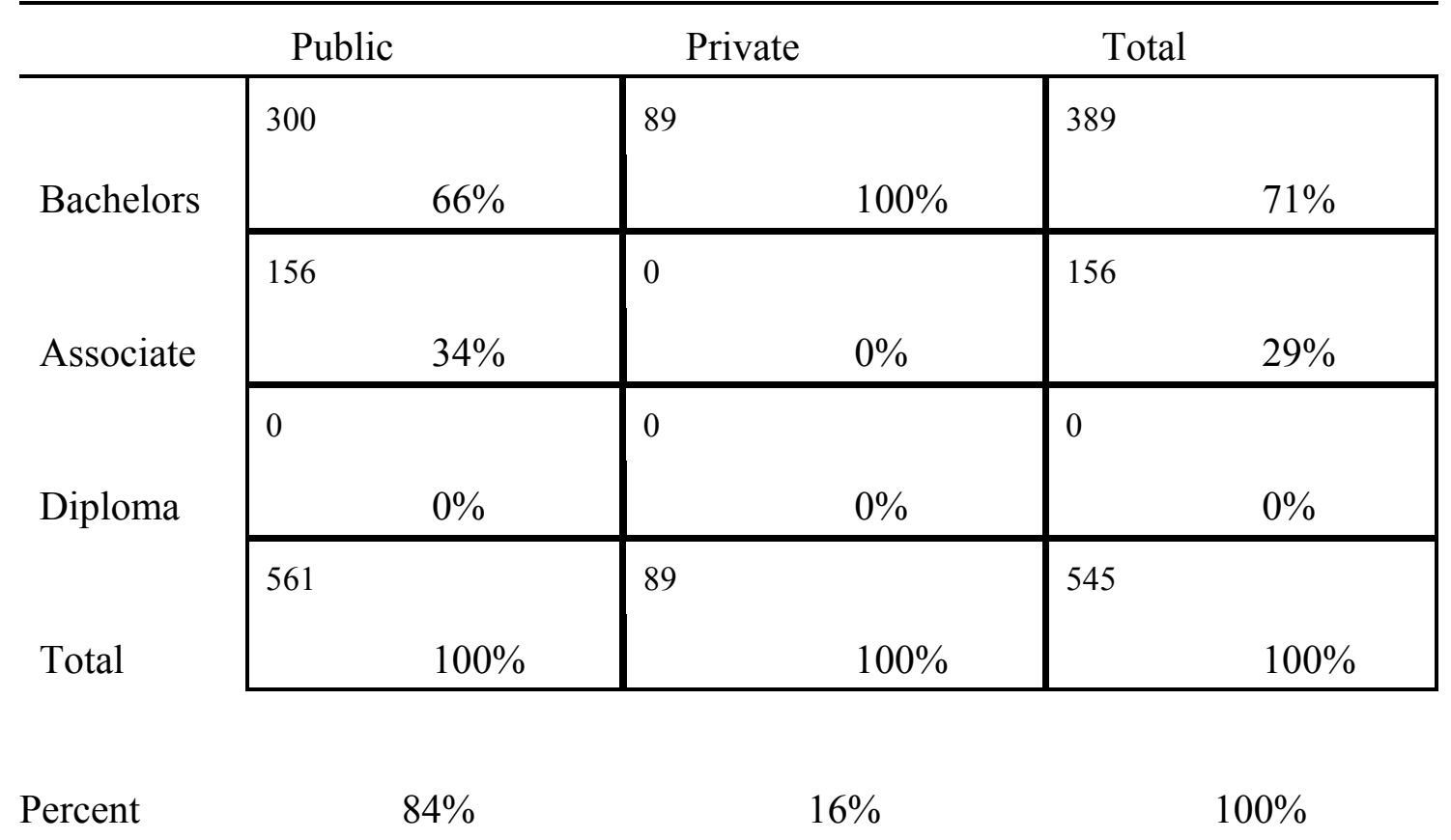


Table B 4. Schools with a Computer Lab in the School of Nursing: Bias-check

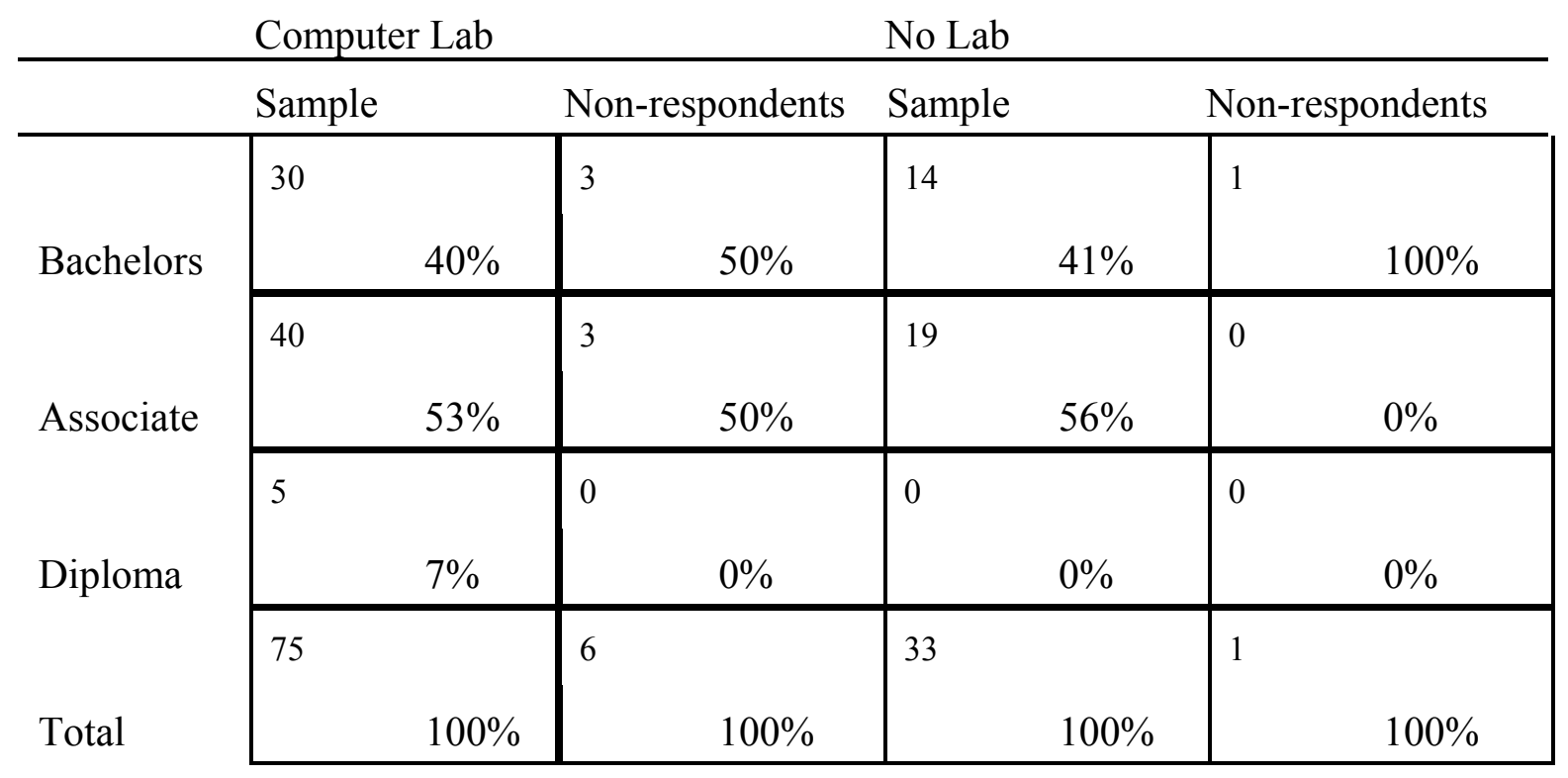

No Response $=1$ (Sample group) 
Table B 5. Participants' Roles: Non-Response Bias-check

\begin{tabular}{|c|c|c|}
\hline Role & Sample & Non-respondents \\
\hline \multirow{3}{*}{ Faculty } & 97 & 3 \\
\hline & $89 \%$ & $43 \%$ \\
\hline & 9 & 4 \\
\hline \multirow[t]{2}{*}{ Administrators } & $8 \%$ & $57 \%$ \\
\hline & 3 & 0 \\
\hline \multirow[t]{2}{*}{ Lab Personnel } & $3 \%$ & $0 \%$ \\
\hline & 109 & 7 \\
\hline Total & $100 \%$ & $100 \%$ \\
\hline
\end{tabular}

Table B 6. Participants' Genders: Non-Response Bias-check

\begin{tabular}{|c|c|c|}
\hline Gender & Sample & Non-respondents \\
\hline \multirow{3}{*}{ Male } & 6 & 7 \\
\hline & $5 \%$ & $100 \%$ \\
\hline & 102 & 0 \\
\hline \multirow[t]{2}{*}{ Female } & $95 \%$ & $0 \%$ \\
\hline & 108 & 7 \\
\hline Total & $100 \%$ & $100 \%$ \\
\hline
\end{tabular}

No Response $=1($ Sample group $)$ 
Table B 7. Participants Age Groups: Non-Response Bias-check

\begin{tabular}{|c|c|c|}
\hline Age Range & Sample & Non-respondents \\
\hline \multirow{3}{*}{20 to 40 years } & 12 & 0 \\
\hline & $11 \%$ & $0 \%$ \\
\hline & 46 & 3 \\
\hline \multirow[t]{2}{*}{41 to 50 years } & $43 \%$ & $43 \%$ \\
\hline & 50 & 4 \\
\hline \multirow[t]{2}{*}{51 and older } & $46 \%$ & $57 \%$ \\
\hline & 108 & 7 \\
\hline Total & $100 \%$ & $100 \%$ \\
\hline
\end{tabular}

No Response $=1$ (Sample group $)$ 
Table B 8. Participants' Educational Backgrounds: Non-Response Bias-check

\begin{tabular}{|c|c|c|}
\hline Education & Sample & Non-respondents \\
\hline \multirow{3}{*}{ BSN and Lower } & 7 & 0 \\
\hline & $6 \%$ & $0 \%$ \\
\hline & 72 & 4 \\
\hline \multirow[t]{2}{*}{ MSN } & $67 \%$ & $57 \%$ \\
\hline & 29 & 3 \\
\hline \multirow[t]{2}{*}{ Doctorate } & $27 \%$ & $29 \%$ \\
\hline & 108 & 6 \\
\hline Total & $100 \%$ & $100 \%$ \\
\hline
\end{tabular}

No Response $=1$ (Sample group), 1 (Non-respondent group) 
Appendix C

Table C 1. Frequency of Use of Integration Strategies: Measures of Central Tendency $(n=77)$

\begin{tabular}{|c|c|c|c|c|}
\hline \multicolumn{2}{|c|}{ Strategy } & \multirow{2}{*}{$\begin{array}{c}\text { Means } \\
1.64\end{array}$} & \multirow{2}{*}{$\frac{\mathrm{SD}}{1.18}$} & \multirow{2}{*}{$\frac{\text { Median }}{1}$} \\
\hline $\mathrm{A}$ & Pretest & & & \\
\hline B & Explain Objectives & 2.66 & 0.99 & 3 \\
\hline $\mathrm{C}$ & Explain CAI & 2.92 & 0.60 & 3 \\
\hline $\mathrm{D}$ & Motivate & 3.05 & 1.01 & 3 \\
\hline $\mathrm{E}$ & Integrate & 3.34 & 1.01 & 3 \\
\hline $\mathrm{F}$ & Individual & 3.56 & 1.00 & 3 \\
\hline G & Small Group & 2.42 & 1.45 & 3 \\
\hline $\mathrm{H}$ & Whole Class & 2.17 & 1.49 & 1 \\
\hline I & Instructional Support & 3.70 & 1.35 & 4 \\
\hline $\mathrm{J}$ & Technical Support & 3.78 & 1.33 & 4 \\
\hline $\mathrm{K}$ & Post Instruction & 2.74 & 1.49 & 3 \\
\hline $\mathrm{L}$ & Follow-up & 2.96 & 1.40 & 3 \\
\hline M & Required & 2.99 & 1.28 & 3 \\
\hline $\mathrm{N}$ & Optional & 2.43 & 1.37 & 3 \\
\hline $\mathrm{O}$ & Course Exam & 2.88 & 1.25 & 3 \\
\hline $\mathrm{P}$ & Embedded & 1.62 & 1.09 & 1 \\
\hline Q & Replace Traditional & 2.35 & 1.37 & 3 \\
\hline $\mathrm{R}$ & Alternative & 1.21 & 1.35 & 3 \\
\hline S & Evaluate CAI & 2.18 & 1.21 & 3 \\
\hline
\end{tabular}


Table C 2. Percentages of Frequency of Use of Integration Strategies $(n=77)$

\begin{tabular}{|c|c|c|c|c|c|c|}
\hline \multicolumn{2}{|c|}{ Strategy } & \multirow{2}{*}{$\frac{1=\text { Never }}{72 \%}$} & \multirow{2}{*}{$\frac{2=\text { Yearly }}{3 \%}$} & \multirow{2}{*}{$\begin{array}{l}3=\text { Semester } \\
18 \%\end{array}$} & \multirow{2}{*}{$\frac{4=\text { Monthly }}{0 \%}$} & \multirow{2}{*}{$\frac{5=\text { Weekly }}{7 \%}$} \\
\hline A & Pretest & & & & & \\
\hline B & Explain Objectives & $16 \%$ & $5 \%$ & $72 \%$ & $3 \%$ & $4 \%$ \\
\hline $\mathrm{C}$ & Explain CAI & $4 \%$ & $8 \%$ & $83 \%$ & $3 \%$ & $3 \%$ \\
\hline $\mathrm{D}$ & Motivate & $12 \%$ & $4 \%$ & $61 \%$ & $14 \%$ & $9 \%$ \\
\hline $\mathrm{E}$ & Integrate & $6 \%$ & $4 \%$ & $56 \%$ & $17 \%$ & $17 \%$ \\
\hline $\mathrm{F}$ & Individual & $4 \%$ & $4 \%$ & $47 \%$ & $23 \%$ & $22 \%$ \\
\hline $\mathrm{G}$ & Small Group & $47 \%$ & $1 \%$ & $26 \%$ & $16 \%$ & $10 \%$ \\
\hline $\mathrm{H}$ & Whole Class & $55 \%$ & $5 \%$ & $14 \%$ & $14 \%$ & $11 \%$ \\
\hline $\mathrm{J}$ & Instructional Support & $6 \%$ & $6 \%$ & $47 \%$ & $6 \%$ & $34 \%$ \\
\hline I & Technical Support & $5 \%$ & $5 \%$ & $38 \%$ & $5 \%$ & $45 \%$ \\
\hline $\mathrm{K}$ & Post Instruction & $32 \%$ & $3 \%$ & $34 \%$ & $16 \%$ & $16 \%$ \\
\hline $\mathrm{L}$ & Follow-up & $23 \%$ & $3 \%$ & $39 \%$ & $20 \%$ & $16 \%$ \\
\hline M & Required & $18 \%$ & $4 \%$ & $51 \%$ & $9 \%$ & $17 \%$ \\
\hline $\mathrm{N}$ & Optional & $38 \%$ & $4 \%$ & $38 \%$ & $11 \%$ & $9 \%$ \\
\hline $\mathrm{O}$ & Course Exam & $22 \%$ & $4 \%$ & $39 \%$ & $28 \%$ & $7 \%$ \\
\hline $\mathrm{P}$ & Embedded & $71 \%$ & $1 \%$ & $24 \%$ & $0 \%$ & $4 \%$ \\
\hline Q & Replace Traditional & $41 \%$ & $4 \%$ & $35 \%$ & $12 \%$ & $8 \%$ \\
\hline $\mathrm{R}$ & Alternative & $47 \%$ & $3 \%$ & $36 \%$ & $7 \%$ & $8 \%$ \\
\hline S & Evaluate CAI & $42 \%$ & $4 \%$ & $45 \%$ & $3 \%$ & $5 \%$ \\
\hline
\end{tabular}

Likert Scale 1 to 5 
Table C 3. Effectiveness of Integration Strategies: Measures of Central Tendency $(n=77)$

\begin{tabular}{|c|c|c|c|c|}
\hline \multicolumn{2}{|c|}{ Strategy } & \multirow{2}{*}{$\frac{\text { Mean }}{2.46}$} & \multirow{2}{*}{$\frac{\mathrm{SD}}{0.94}$} & \multirow{2}{*}{$\frac{\text { Median }}{3}$} \\
\hline A & Pretest & & & \\
\hline $\mathrm{B}$ & Explain Objectives & 3.20 & 0.89 & 3 \\
\hline $\mathrm{C}$ & Explain CAI & 3.34 & 0.80 & 3 \\
\hline $\mathrm{D}$ & Motivate & 3.32 & 0.78 & 3 \\
\hline $\mathrm{E}$ & Integrate & 3.26 & 0.70 & 3 \\
\hline $\mathrm{F}$ & Individual & 3.28 & 0.62 & 3 \\
\hline $\mathrm{G}$ & Small Group & 2.83 & 0.83 & 3 \\
\hline $\mathrm{H}$ & Whole Class & 2.72 & 0.88 & 3 \\
\hline I & Instructional Support & 3.58 & 0.73 & 4 \\
\hline $\mathrm{J}$ & Technical Support & 3.59 & 0.59 & 4 \\
\hline K & Post Instruction & 3.13 & 0.71 & 3 \\
\hline $\mathrm{L}$ & Follow-up & 3.08 & 0.81 & 3 \\
\hline M & Required & 3.22 & 0.75 & 3 \\
\hline $\mathrm{N}$ & Optional & 2.75 & 0.73 & 3 \\
\hline $\mathrm{O}$ & Course Exam & 3.15 & 0.81 & 3 \\
\hline $\mathrm{P}$ & Embedded & 2.47 & 0.94 & 3 \\
\hline Q & Replace Traditional & 2.77 & 0.74 & 3 \\
\hline $\mathrm{R}$ & Alternative & 2.92 & 0.73 & 3 \\
\hline S & Evaluate CAI & 2.99 & 0.72 & 3 \\
\hline
\end{tabular}

Likert Scale 1 to 4 
Table C 4. Percentages of Perceived Effectiveness of Integration Strategies to Increase Student Learning $(n=77)$

\begin{tabular}{|c|c|c|c|c|c|}
\hline \multicolumn{2}{|c|}{ Strategy } & \multirow{2}{*}{$\begin{array}{l}1=\text { Strongly } \\
\text { Disagree }\end{array}$} & \multirow{2}{*}{$\frac{2=\text { Disagree }}{31 \%}$} & \multirow{2}{*}{$\frac{3=\text { Agree }}{56 \%}$} & \multirow{2}{*}{$\begin{array}{l}\begin{array}{l}4=\text { Strongly } \\
\text { Agree }\end{array} \\
8 \%\end{array}$} \\
\hline A & Pretest & & & & \\
\hline B & Explain Objectives & $1 \%$ & $4 \%$ & $55 \%$ & $40 \%$ \\
\hline $\mathrm{C}$ & Explain CAI & $1 \%$ & $1 \%$ & $50 \%$ & $47 \%$ \\
\hline $\mathrm{D}$ & Motivate & $1 \%$ & $0 \%$ & $55 \%$ & $43 \%$ \\
\hline E & Integrate & $1 \%$ & $3 \%$ & $60 \%$ & $36 \%$ \\
\hline $\mathrm{F}$ & Individual & $1 \%$ & $5 \%$ & $59 \%$ & $35 \%$ \\
\hline G & Small Group & $4 \%$ & $16 \%$ & $63 \%$ & $16 \%$ \\
\hline $\mathrm{H}$ & Whole Class & $5 \%$ & $26 \%$ & $52 \%$ & $16 \%$ \\
\hline I & Technical Support & $1 \%$ & $3 \%$ & $28 \%$ & $68 \%$ \\
\hline $\mathrm{J}$ & Instructional Support & $! \%$ & $1 \%$ & $34 \%$ & $63 \%$ \\
\hline K & Post Instruction & $1 \%$ & $8 \%$ & $63 \%$ & $28 \%$ \\
\hline $\mathrm{L}$ & Follow-up & $1 \%$ & $9 \%$ & $61 \%$ & $28 \%$ \\
\hline M & Required & $1 \%$ & $9 \%$ & $46 \%$ & $39 \%$ \\
\hline $\mathrm{N}$ & Optional & $7 \%$ & $23 \%$ & $60 \%$ & $11 \%$ \\
\hline $\mathrm{O}$ & Course Exam & $3 \%$ & $11 \%$ & $51 \%$ & $35 \%$ \\
\hline $\mathrm{P}$ & Embedded & $11 \%$ & $38 \%$ & $37 \%$ & $14 \%$ \\
\hline Q & Replace Traditional & $7 \%$ & $21 \%$ & $60 \%$ & $12 \%$ \\
\hline $\mathrm{R}$ & Alternative & $1 \%$ & $19 \%$ & $62 \%$ & $18 \%$ \\
\hline $\mathrm{S}$ & Evaluate CAI & $1 \%$ & $15 \%$ & $64 \%$ & $20 \%$ \\
\hline
\end{tabular}

Likert Scale 1 to 4 
Table C 5. Perceived Effectiveness of Integration Strategies for Decreasing Costs: Measures of Central Tendency $(\mathrm{n}=77)$

\begin{tabular}{llccc}
\hline \multicolumn{2}{l}{ Strategy } & Mean & SD & Median \\
\hline F & Individual & 2.69 & 0.83 & 3 \\
G & Small Group & 2.39 & 0.89 & 3 \\
$\mathrm{H}$ & Whole Class & 2.28 & 0.79 & 2 \\
$\mathrm{I}$ & Instructional Support & 2.56 & 0.97 & 2 \\
$\mathrm{~J}$ & Technical Support & 2.57 & 0.98 & 2 \\
\hline $\mathrm{M}$ & Required & 2.55 & 0.87 & 3 \\
$\mathrm{~N}$ & Optional & 2.32 & 0.71 & 2 \\
$\mathrm{Q}$ & Replace Traditional & 2.61 & 0.88 & 3
\end{tabular}

Likert Scale 1 to 4 
Table C 6. Percentages of Perceived Effectiveness of Integration Strategies for Decreasing Costs $(\mathrm{n}=77)$

\begin{tabular}{|c|c|c|c|c|c|}
\hline \multicolumn{2}{|c|}{ Strategy } & \multirow{2}{*}{$\begin{array}{c}\begin{array}{c}1=\text { Strongly } \\
\text { Disagree }\end{array} \\
4 \%\end{array}$} & \multirow{2}{*}{$\frac{2=\text { Disagree }}{35 \%}$} & \multirow{2}{*}{$\frac{3=\text { Agree }}{45 \%}$} & \multirow{2}{*}{$\begin{array}{c}\begin{array}{l}4=\text { Strongly } \\
\text { Agree }\end{array} \\
16 \%\end{array}$} \\
\hline $\mathrm{F}$ & Individual & & & & \\
\hline G & Small Group & $4 \%$ & $51 \%$ & $41 \%$ & $4 \%$ \\
\hline $\mathrm{H}$ & Whole Class & $8 \%$ & $55 \%$ & $32 \%$ & $5 \%$ \\
\hline $\mathrm{J}$ & Instructional Support & $9 \%$ & $43 \%$ & $26 \%$ & $22 \%$ \\
\hline I & Technical Support & $9 \%$ & $43 \%$ & $24 \%$ & $23 \%$ \\
\hline M & Required & $5 \%$ & $40 \%$ & $42 \%$ & $12 \%$ \\
\hline $\mathrm{N}$ & Optional & $7 \%$ & $55 \%$ & $34 \%$ & $4 \%$ \\
\hline Q & Replace Traditional & $5 \%$ & $34 \%$ & $47 \%$ & $14 \%$ \\
\hline
\end{tabular}

Likert Scale 1 to 4 
Table C 7. Perceived Effectiveness of Integration Strategies for Decreasing Students' Time: Measures of Central Tendency $(\mathrm{n}=77)$

\begin{tabular}{llccc}
\hline \multicolumn{2}{l}{ Strategy } & Mean & SD & Median \\
\hline B & Explain Objectives & 2.99 & 0.76 & 3 \\
C & Explain CAI & 3.19 & 0.71 & 3 \\
\hline F & Individual & 2.88 & 0.69 & 3 \\
G & Small Group & 2.45 & 0.75 & 2 \\
H & Whole Class & 2.55 & 0.82 & 3 \\
I & Instructional Support & 3.39 & 0.63 & 3 \\
J & Technical Support & 3.39 & 0.69 & 3
\end{tabular}

Likert Scale 1 to 4 
Table C 8. Percentages of Perceived Effectiveness of Integration Strategies to Decrease Students' Time $(\mathrm{n}=77)$

\begin{tabular}{|c|c|c|c|c|c|}
\hline \multicolumn{2}{|c|}{ Strategy } & \multirow{2}{*}{$\begin{array}{l}\begin{array}{l}1=\text { Strongly } \\
\text { Disagree }\end{array} \\
1 \%\end{array}$} & \multirow{2}{*}{$\begin{array}{l}2=\text { Disagree } \\
18 \%\end{array}$} & \multirow{2}{*}{$\begin{array}{l}3=\text { Agree } \\
58 \%\end{array}$} & \multirow{2}{*}{$\begin{array}{c}\begin{array}{c}4=\text { Strongly } \\
\text { Agree }\end{array} \\
23 \%\end{array}$} \\
\hline B & Explain Objectives & & & & \\
\hline $\mathrm{C}$ & Explain CAI & $1 \%$ & $5 \%$ & $62 \%$ & $31 \%$ \\
\hline $\mathrm{F}$ & Individual & $3 \%$ & $24 \%$ & $63 \%$ & $10 \%$ \\
\hline G & Small Group & $3 \%$ & $48 \%$ & $44 \%$ & $5 \%$ \\
\hline $\mathrm{H}$ & Whole Class & $6 \%$ & $43 \%$ & $40 \%$ & $11 \%$ \\
\hline I & Technical Support & $1 \%$ & $4 \%$ & $49 \%$ & $46 \%$ \\
\hline $\mathrm{J}$ & Instructional Support & $1 \%$ & $4 \%$ & $49 \%$ & $46 \%$ \\
\hline
\end{tabular}

Likert Scale 1 to 4 
Table C 9. Perceived Effectiveness of Integration Strategies for Decreasing Teachers' Time: Measures of Central Tendency $(\mathrm{n}=77)$

\begin{tabular}{llllc}
\hline \multicolumn{2}{l}{ Strategy } & Mean & SD & Median \\
\hline F & Individual & 3.00 & 0.90 & 3 \\
G & Small Group & 2.61 & 0.87 & 3 \\
$\mathrm{H}$ & Whole Class & 2.45 & 0.90 & 2 \\
$\mathrm{I}$ & Instructional Support & 3.20 & 0.99 & 4 \\
$\mathrm{~J}$ & Technical Support & 3.05 & 1.01 & 3 \\
\hline $\mathrm{M}$ & Required & 2.70 & 1.01 & 3 \\
$\mathrm{~N}$ & Optional & 2.47 & 0.77 & 2 \\
$\mathrm{O}$ & Course Exam & 2.59 & 1.01 & 3 \\
$\mathrm{P}$ & Embedded & 2.39 & 0.96 & 2 \\
$\mathrm{Q}$ & Replace Traditional & 2.82 & 0.92 & 3
\end{tabular}

Likert Scale 1 to 4 
Table C 10. Percentages of Perceived Effectiveness of Integration Strategies to Decrease Teachers' Time $(\mathrm{n}=77)$

\begin{tabular}{|c|c|c|c|c|c|}
\hline \multicolumn{2}{|c|}{ Strategy } & \multicolumn{2}{|l|}{$1=$ Strongly } & \multirow{2}{*}{$\begin{array}{l}3=\text { Agree } \\
42 \%\end{array}$} & \multirow{2}{*}{$\begin{array}{c}\begin{array}{c}4=\text { Strongly } \\
\text { Agree }\end{array} \\
33 \%\end{array}$} \\
\hline $\mathrm{F}$ & Individual & $4 \%$ & $21 \%$ & & \\
\hline $\mathrm{G}$ & Small Group & $4 \%$ & $38 \%$ & $46 \%$ & $13 \%$ \\
\hline $\mathrm{H}$ & Whole Class & $12 \%$ & $40 \%$ & $36 \%$ & $12 \%$ \\
\hline $\mathrm{J}$ & Instructional Support & $7 \%$ & $12 \%$ & $30 \%$ & $51 \%$ \\
\hline I & Technical Support & $8 \%$ & $16 \%$ & $33 \%$ & $42 \%$ \\
\hline M & Required & $7 \%$ & $27 \%$ & $43 \%$ & $22 \%$ \\
\hline $\mathrm{N}$ & Optional & $4 \%$ & $51 \%$ & $36 \%$ & $10 \%$ \\
\hline $\mathrm{O}$ & Course Exam & $7 \%$ & $37 \%$ & $35 \%$ & $21 \%$ \\
\hline $\mathrm{P}$ & Embedded & $10 \%$ & $44 \%$ & $34 \%$ & $13 \%$ \\
\hline Q & Replace Traditional & $6 \%$ & $22 \%$ & $39 \%$ & $34 \%$ \\
\hline
\end{tabular}

Likert Scale 1 to 4 
Table C 11. Comparison of Means of Frequency of Use of Integration Strategies by Type of School $(n=77)$

\begin{tabular}{llccc}
\hline Strategy & & $\mathrm{BSN}(\mathrm{n}=25)$ & $\mathrm{AD}(\mathrm{n}=48)$ & Diploma $(\mathrm{n}=4)$ \\
\hline $\mathrm{A}$ & Pretest & 1.60 & 1.71 & 1.00 \\
$\mathrm{~B}$ & Explain Objectives & 2.68 & 2.75 & 1.50 \\
$\mathrm{C}$ & Explain CAI & 3.00 & 2.94 & 2.25 \\
$\mathrm{D}$ & Motivate & 3.04 & 3.06 & 3.00 \\
$\mathrm{E}$ & Integrate & 3.54 & 3.29 & 2.75 \\
\hline $\mathrm{F}$ & Individual & 3.60 & 3.63 & 2.50 \\
$\mathrm{G}$ & Small Group & 2.52 & 2.44 & 1.50 \\
$\mathrm{H}$ & Whole Class & 2.52 & 2.08 & 1.00 \\
$\mathrm{I}$ & Technical Support & 3.68 & 3.79 & 2.75 \\
$\mathrm{~J}$ & Instructional Support & 3.68 & 3.90 & 3.00 \\
\hline $\mathrm{K}$ & Post Instruction & 2.80 & 2.73 & 2.50 \\
$\mathrm{~L}$ & Follow-up & 3.36 & 2.92 & 1.00 \\
$\mathrm{M}$ & Required & 2.96 & 3.02 & 2.75 \\
$\mathrm{~N}$ & Optional & 2.28 & 2.50 & 2.50 \\
$\mathrm{O}$ & Course Exam & 2.68 & 3.10 & 1.50 \\
$\mathrm{P}$ & Embedded & 1.80 & 1.48 & 2.25 \\
$\mathrm{Q}$ & Replace Traditional & 2.52 & 2.38 & 1.00 \\
$\mathrm{R}$ & Alternative & 1.72 & 2.44 & 2.50 \\
$\mathrm{~S}$ & Evaluate CAI & 2.36 & 2.15 & 1.50
\end{tabular}

Likert Scale 1 to 5 
Table C 12. Comparison of Frequency of Use of Integration Strategies based on Type of Institution $(\mathrm{n}=77)$

\begin{tabular}{|c|c|c|c|}
\hline \multicolumn{2}{|c|}{ Strategy } & \multirow{2}{*}{$\frac{\text { Public }(n=60)}{1.77}$} & \multirow{2}{*}{$\frac{\text { Private }(n=17)}{1.18}$} \\
\hline A & Pretest & & \\
\hline $\mathrm{B}$ & Explain Objectives & 2.70 & 2.52 \\
\hline $\mathrm{C}$ & Explain CAI & 2.95 & 2.82 \\
\hline $\mathrm{D}$ & Motivate & 3.13 & 2.76 \\
\hline $\mathrm{E}$ & Integrate & 3.37 & 3.24 \\
\hline $\mathrm{F}$ & Individual & 3.60 & 3.41 \\
\hline G & Small Group & 2.52 & 2.06 \\
\hline $\mathrm{H}$ & Whole Class & 2.22 & 2.00 \\
\hline I & Technical Support & 3.80 & 3.35 \\
\hline $\mathrm{J}$ & Instructional Support & 3.88 & 3.41 \\
\hline $\mathrm{K}$ & Post Instruction & 2.83 & 2.41 \\
\hline $\mathrm{L}$ & Follow-up & 2.97 & 2.94 \\
\hline M & Required & 2.92 & 3.24 \\
\hline $\mathrm{N}$ & Optional & 2.47 & 2.29 \\
\hline $\mathrm{O}$ & Course Exam & 2.90 & 2.82 \\
\hline $\mathrm{P}$ & Embedded & 1.62 & 1.65 \\
\hline Q & Replace Traditional & 2.42 & 2.12 \\
\hline $\mathrm{R}$ & Alternative & 2.25 & 1.06 \\
\hline S & Evaluate CAI & 2.20 & 2.12 \\
\hline
\end{tabular}

Likert Scale 1 to 5 
Table C 13. Comparison of Frequency of Use of Integration Strategies based on School Sizes $(n=77)$

\begin{tabular}{|c|c|c|c|c|}
\hline \multicolumn{2}{|c|}{ Strategy } & \multirow{2}{*}{$\frac{\text { Small }(n=25)}{1.50}$} & \multirow{2}{*}{$\frac{\text { Medium }(n=28)}{1.59}$} & \multirow{2}{*}{$\frac{\text { Large }(n=24)}{1.83}$} \\
\hline $\mathrm{A}$ & Pretest & & & \\
\hline $\mathrm{B}$ & Explain Objectives & 2.35 & 2.96 & 2.67 \\
\hline $\mathrm{C}$ & Explain CAI & 2.65 & 2.96 & 3.17 \\
\hline $\mathrm{D}$ & Motivate & 2.88 & 3.22 & 3.04 \\
\hline $\mathrm{E}$ & Integrate & 3.04 & 3.59 & 3.38 \\
\hline $\mathrm{F}$ & Individual & 3.27 & 3.78 & 3.63 \\
\hline $\mathrm{G}$ & Small Group & 1.85 & 2.78 & 2.63 \\
\hline $\mathrm{H}$ & Whole Class & 2.08 & 2.41 & 2.00 \\
\hline I & Technical Support & 3.38 & 3.81 & 3.92 \\
\hline $\mathrm{J}$ & Instructional Support & 3.58 & 3.85 & 3.92 \\
\hline $\mathrm{K}$ & Post Instruction & 2.35 & 2.85 & 3.04 \\
\hline $\mathrm{L}$ & Follow-up & 2.23 & 3.48 & 3.17 \\
\hline M & Required & 2.69 & 3.00 & 3.29 \\
\hline $\mathrm{N}$ & Optional & 2.19 & 2.74 & 2.33 \\
\hline $\mathrm{O}$ & Course Exam & 2.46 & 3.04 & 3.17 \\
\hline $\mathrm{P}$ & Embedded & 1.54 & 1.59 & 1.75 \\
\hline Q & Replace Traditional & 1.73 & 2.59 & 2.75 \\
\hline $\mathrm{R}$ & Alternative & 2.12 & 2.07 & 2.46 \\
\hline S & Evaluate CAI & 1.73 & 2.30 & 2.54 \\
\hline
\end{tabular}

Likert Scale 1 to 5 
Table C 14. Comparison of Perceived Effectiveness of Integration Strategies to Increase Student Learning based on Type of School $(n=77)$

\begin{tabular}{llccc}
\hline Strategy & & $\mathrm{BSN}(\mathrm{n}=25)$ & $\mathrm{AD}(\mathrm{n}=48)$ & Diploma $(\mathrm{n}=4)$ \\
\hline $\mathrm{A}$ & Pretest & 2.58 & 2.46 & 1.75 \\
$\mathrm{~B}$ & Explain Objectives & 3.33 & 3.17 & 2.75 \\
$\mathrm{C}$ & Explain CAI & 3.48 & 3.33 & 2.75 \\
$\mathrm{D}$ & Motivate & 3.25 & 3.38 & 2.75 \\
$\mathrm{E}$ & Integrate & 3.25 & 3.13 & 2.75 \\
\hline $\mathrm{F}$ & Individual & 3.21 & 3.27 & 2.75 \\
$\mathrm{G}$ & Small Group & 2.75 & 2.90 & 2.00 \\
$\mathrm{H}$ & Whole Class & 2.79 & 2.69 & 2.00 \\
$\mathrm{I}$ & Technical Support & 3.63 & 3.63 & 2.75 \\
$\mathrm{~J}$ & Instructional Support & 3.58 & 3.71 & 2.25 \\
\hline $\mathrm{K}$ & Post Instruction & 3.21 & 3.17 & 2.25 \\
$\mathrm{~L}$ & Follow-up & 3.13 & 4.15 & 2.00 \\
$\mathrm{M}$ & Required & 3.25 & 3.27 & 2.50 \\
$\mathrm{~N}$ & Optional & 2.57 & 2.88 & 2.25 \\
$\mathrm{O}$ & Course Exam & 3.22 & 3.21 & 2.00 \\
$\mathrm{P}$ & Embedded & 2.74 & 2.31 & 2.75 \\
$\mathrm{Q}$ & Replace Traditional & 3.00 & 2.77 & 1.50 \\
$\mathrm{R}$ & Alternative & 2.96 & 2.96 & 2.25 \\
$\mathrm{~S}$ & Evaluate CAI & 3.04 & 3.04 & 2.00
\end{tabular}

Likert Scale 1 to 4 
Table C 15. Comparison of the Perceived Effectiveness of the Integration Strategies to Increase Student Learning based on Type of Institution $(n=77)$

\begin{tabular}{llcc}
\hline Strategy & & Public $(\mathrm{n}=60)$ & Private $(\mathrm{n}=17)$ \\
\hline $\mathrm{A}$ & Pretest & 2.49 & 2.35 \\
$\mathrm{~B}$ & Explain Objectives & 3.24 & 3.06 \\
$\mathrm{C}$ & Explain CAI & 3.36 & 3.29 \\
$\mathrm{D}$ & Motivate & 3.36 & 3.18 \\
$\mathrm{E}$ & Integrate & 3.27 & 3.24 \\
\hline $\mathrm{F}$ & Individual & 3.27 & 3.06 \\
$\mathrm{G}$ & Small Group & 2.80 & 2.82 \\
$\mathrm{H}$ & Whole Class & 2.68 & 2.71 \\
$\mathrm{I}$ & Technical Support & 3.64 & 3.35 \\
$\mathrm{~J}$ & Instructional Support & 3.69 & 3.24 \\
\hline $\mathrm{K}$ & Post Instruction & 3.20 & 2.88 \\
$\mathrm{~L}$ & Follow-up & 3.19 & 2.71 \\
$\mathrm{M}$ & Required & 3.25 & 3.12 \\
$\mathrm{~N}$ & Optional & 2.83 & 2.44 \\
$\mathrm{O}$ & Course Exam & 3.17 & 3.06 \\
$\mathrm{P}$ & Embedded & 2.51 & 2.31 \\
$\mathrm{Q}$ & Replace Traditional & 2.83 & 2.56 \\
$\mathrm{R}$ & Alternative & 2.92 & 2.94 \\
$\mathrm{~S}$ & Evaluate CAI & 3.03 & 2.81
\end{tabular}

Likert Scale 1 to 4 
Table C 16. Comparison of the Perceived Effectiveness of Integration Strategies to Increase Student Learning based on School Size $(\mathrm{n}=77)$

\begin{tabular}{llccc}
\hline Strategy & & Small $(\mathrm{n}=25)$ & Medium $(\mathrm{n}=28)$ & Large $(\mathrm{n}=24)$ \\
\hline $\mathrm{A}$ & Pretest & 2.04 & 2.69 & 2.67 \\
$\mathrm{~B}$ & Explain Objectives & 2.85 & 3.38 & 3.38 \\
$\mathrm{C}$ & Explain CAI & 3.15 & 3.42 & 3.46 \\
$\mathrm{D}$ & Motivate & 3.08 & 3.42 & 3.46 \\
$\mathrm{E}$ & Integrate & 3.08 & 3.46 & 3.25 \\
\hline $\mathrm{F}$ & Individual & 3.08 & 3.27 & 3.33 \\
$\mathrm{G}$ & Small Group & 2.46 & 3.00 & 2.96 \\
$\mathrm{H}$ & Whole Class & 2.69 & 2.73 & 2.63 \\
$\mathrm{I}$ & Technical Support & 3.54 & 3.73 & 3.46 \\
$\mathrm{~J}$ & Instructional Support & 3.54 & 3.65 & 3.58 \\
\hline $\mathrm{K}$ & Post Instruction & 2.92 & 3.23 & 3.25 \\
$\mathrm{~L}$ & Follow-up & 2.85 & 3.23 & 3.17 \\
$\mathrm{M}$ & Required & 3.27 & 3.23 & 3.17 \\
$\mathrm{~N}$ & Optional & 2.73 & 2.76 & 2.75 \\
$\mathrm{O}$ & Course Exam & 3.04 & 3.12 & 3.29 \\
$\mathrm{P}$ & Embedded & 2.23 & 2.56 & 2.63 \\
$\mathrm{Q}$ & Replace Traditional & 2.58 & 2.88 & 2.88 \\
$\mathrm{R}$ & Alternative & 2.69 & 2.92 & 3.17 \\
$\mathrm{~S}$ & Evaluate CAI & 2.92 & 3.04 & 3.00
\end{tabular}

Likert Scale 1 to 4 
Table C 17. Comparison of Perceived Effectiveness of Integration Strategies for Decreasing Costs based on Type of School $(\mathrm{n}=77)$

\begin{tabular}{lllll}
\hline $\begin{array}{l}\text { Strategy } \\
\text { 4) }\end{array}$ & & $\mathrm{BSN}(\mathrm{n}=25)$ & $\mathrm{AD}(\mathrm{n}=48)$ & Diploma $(\mathrm{n}=$ \\
\hline $\mathrm{F}$ & Individual & 2.65 & 2.77 & 2.00 \\
$\mathrm{G}$ & Small Group & 2.35 & 2.44 & 2.00 \\
$\mathrm{H}$ & Whole Class & 2.35 & 2.31 & 1.50 \\
$\mathrm{I}$ & Technical Support & 2.48 & 2.67 & 1.75 \\
$\mathrm{~J}$ & Instructional Support & 2.48 & 2.69 & 1.75 \\
\hline $\mathrm{M}$ & Required & 2.61 & 2.56 & 2.00 \\
$\mathrm{~N}$ & Optional & 2.26 & 2.35 & 2.25 \\
$\mathrm{Q}$ & Replace Traditional & 2.74 & 2.65 & 1.50
\end{tabular}

Likert Scale 1 to 4 
Table C 18. Comparison of Perceived Effectiveness of Integration Strategies for Decreasing Costs based on Type of Institution ( $\mathrm{n}=77)$

\begin{tabular}{llcc}
\hline Strategy & & Public $(\mathrm{n}=60)$ & Private $(\mathrm{n}=17)$ \\
\hline $\mathrm{F}$ & Individual & 2.76 & 2.44 \\
$\mathrm{G}$ & Small Group & 2.44 & 2.19 \\
$\mathrm{H}$ & Whole Class & 2.29 & 2.25 \\
$\mathrm{I}$ & Technical Support & 2.68 & 2.13 \\
$\mathrm{~J}$ & Instructional Support & 2.69 & 2.13 \\
\hline $\mathrm{M}$ & Required & 2.61 & 2.31 \\
$\mathrm{~N}$ & Optional & 2.34 & 2.25 \\
$\mathrm{Q}$ & Replace Traditional & 2.68 & 2.38
\end{tabular}

Likert Scale 1 to 4 
Table C 19. Comparison of Perceived Effectiveness of Integration Strategies for Decreasing Costs based on School Sizes $(n=77)$

\begin{tabular}{lllll}
\hline Strategy & \multicolumn{1}{c}{$(\mathrm{n}=24)$} & Small $(\mathrm{n}=25)$ & Medium $(\mathrm{n}=28)$ & Large \\
\hline $\mathrm{F}$ & Individual & 2.54 & 2.68 & 2.88 \\
$\mathrm{G}$ & Small Group & 2.12 & 2.52 & 2.54 \\
$\mathrm{H}$ & Whole Class & 2.15 & 2.32 & 2.38 \\
$\mathrm{I}$ & Technical Support & 2.23 & 2.60 & 2.88 \\
$\mathrm{~J}$ & Instructional Support & 2.27 & 2.60 & 2.88 \\
\hline $\mathrm{M}$ & Required & 2.46 & 2.44 & 2.75 \\
$\mathrm{~N}$ & Optional & 2.12 & 2.20 & 2.67 \\
$\mathrm{Q}$ & Replace Traditional & 2.27 & 2.80 & 2.79
\end{tabular}

Likert Scale 1 to 4 
Table C 20. Comparison of Perceived Effectiveness of Integration Strategies for Decreasing Students' Time based on Type of School $(n=77)$

\begin{tabular}{lllll}
\hline $\begin{array}{l}\text { Strategy } \\
\text { 4) }\end{array}$ & & BSN $(\mathrm{n}=25)$ & $\mathrm{AD}(\mathrm{n}=48)$ & Diploma $(\mathrm{n}=$ \\
\hline B & Explain Objectives & 3.09 & 3.00 & 2.25 \\
$\mathrm{C}$ & Explain CAI & 3.35 & 3.19 & 2.25 \\
\hline F & Individual & 2.87 & 2.94 & 2.25 \\
G & Small Group & 2.35 & 2.54 & 2.00 \\
H & Whole Class & 2.70 & 2.53 & 2.00 \\
I & Instructional Support & 3.43 & 3.47 & 2.25 \\
J & Technical Support & 3.43 & 3.47 & 2.25
\end{tabular}

Likert Scale 1 to 4 
Table C 21. Comparison of Perceived Effectiveness of Integration Strategies for Decreasing Students' Time based on Type of Institution $(n=77)$

\begin{tabular}{llcc}
\hline Strategy & & Public $(\mathrm{n}=60)$ & Private $(\mathrm{n}=17)$ \\
\hline B & Explain Objectives & 3.00 & 2.94 \\
C & Explain CAI & 3.17 & 3.25 \\
\hline F & Individual & 2.92 & 2.75 \\
G & Small Group & 2.53 & 2.19 \\
H & Whole Class & 2.55 & 2.56 \\
I & Instructional Support & 3.45 & 3.19 \\
J & Technical Support & 3.45 & 3.19
\end{tabular}

Likert Scale 1 to 4 
Table C 22. Comparison of Perceived Effectiveness of Integration Strategies for Decreasing Students' Time based on Size of Schools $(\mathrm{n}=77)$

\begin{tabular}{llccc}
\hline Strategy & & Small $(\mathrm{n}=25)$ & Medium $(\mathrm{n}=28)$ & Large $(\mathrm{n}=24)$ \\
\hline $\mathrm{B}$ & Explain Objectives & 2.77 & 3.08 & 3.13 \\
$\mathrm{C}$ & Explain CAI & 3.00 & 3.36 & 3.21 \\
\hline $\mathrm{F}$ & Individual & 2.73 & 2.80 & 3.13 \\
$\mathrm{G}$ & Small Group & 2.23 & 2.68 & 2.46 \\
$\mathrm{H}$ & Whole Class & 2.56 & 2.72 & 2.38 \\
$\mathrm{I}$ & Instructional Support & 3.24 & 3.52 & 3.42 \\
$\mathrm{~J}$ & Technical Support & 3.24 & 3.52 & 3.42
\end{tabular}

Likert Scale 1 to 4 
Table C 23. Comparison of Perceived Effectiveness of Integration Strategies for Decreasing Teachers' Time based on Type of School $(\mathrm{n}=77)$

\begin{tabular}{llccc}
\hline Strategy & & $\mathrm{BSN}(\mathrm{n}=25)$ & $\mathrm{AD}(\mathrm{n}=48)$ & Diploma $(\mathrm{n}=4)$ \\
\hline $\mathrm{F}$ & Individual & 3.04 & 3.00 & 2.75 \\
$\mathrm{G}$ & Small Group & 2.70 & 2.60 & 2.25 \\
$\mathrm{H}$ & Whole Class & 2.35 & 2.51 & 2.25 \\
$\mathrm{I}$ & Technical Support & 3.52 & 3.13 & 2.25 \\
$\mathrm{~J}$ & Instructional Support & 3.30 & 3.00 & 2.25 \\
\hline $\mathrm{M}$ & Required & 2.91 & 2.64 & 2.25 \\
$\mathrm{~N}$ & Optional & 2.39 & 2.49 & 2.75 \\
$\mathrm{O}$ & Course Exam & 3.04 & 2.51 & 1.00 \\
$\mathrm{P}$ & Embedded & 2.74 & 2.21 & 2.50 \\
$\mathrm{Q}$ & Replace Traditional & 2.96 & 2.85 & 1.75 \\
\hline
\end{tabular}

Likert Scale 1 to 4 
Table C 24. Comparison of Perceived Effectiveness of Integration Strategies for Decreasing Teachers' Time based on Type of Institution $(n=77)$

\begin{tabular}{llcc}
\hline Strategy & & Public $(\mathrm{n}=60)$ & Private $(\mathrm{n}=17)$ \\
\hline $\mathrm{F}$ & Individual & 3.03 & 2.88 \\
$\mathrm{G}$ & Small Group & 2.62 & 2.56 \\
$\mathrm{H}$ & Whole Class & 2.45 & 2.44 \\
$\mathrm{I}$ & Technical Support & 3.19 & 3.25 \\
$\mathrm{~J}$ & Instructional Support & 3.00 & 3.25 \\
\hline $\mathrm{M}$ & Required & 2.72 & 2.63 \\
$\mathrm{~N}$ & Optional & 2.48 & 2.44 \\
$\mathrm{O}$ & Course Exam & 2.62 & 2.50 \\
$\mathrm{P}$ & Embedded & 2.36 & 2.50 \\
$\mathrm{Q}$ & Replace Traditional & 2.91 & 2.50
\end{tabular}

Likert Scale 1 to 4 
Table C 25. Comparison of Perceived Effectiveness of Integration Strategies for Decreasing Teachers' Time based on Size of School $(\mathrm{n}=77)$

\begin{tabular}{llccc}
\hline Strategy & & Small $(\mathrm{n}=25)$ & Medium $(\mathrm{n}=28)$ & Large $(\mathrm{n}=24)$ \\
\hline $\mathrm{F}$ & Individual & 2.92 & 3.12 & 2.96 \\
$\mathrm{G}$ & Small Group & 2.40 & 2.84 & 2.58 \\
$\mathrm{H}$ & Whole Class & 2.44 & 2.56 & 2.33 \\
$\mathrm{I}$ & Technical Support & 2.84 & 3.36 & 3.42 \\
$\mathrm{~J}$ & Instructional Support & 2.64 & 3.24 & 3.29 \\
\hline $\mathrm{M}$ & Required & 2.60 & 2.84 & 2.67 \\
$\mathrm{~N}$ & Optional & 2.56 & 2.36 & 2.50 \\
$\mathrm{O}$ & Course Exam & 2.36 & 2.64 & 2.79 \\
$\mathrm{P}$ & Embedded & 2.24 & 2.60 & 2.33 \\
$\mathrm{Q}$ & Replace Traditional & 2.76 & 3.04 & 2.67
\end{tabular}

Likert Scale 1 to 4 
Table C 26. Comparison of Correlations Between Frequency of Use and Perceived Effectiveness of Integration Strategies for Increasing Student Learning by Type of School $(\mathrm{n}=77)$

\begin{tabular}{|c|c|c|c|}
\hline Strategy & & $\mathrm{BSN}(\mathrm{n}=25)$ & $\mathrm{AD}(\mathrm{n}=48)$ \\
\hline A & Pretest & $0.43 *$ & $0.50 *$ \\
\hline B & Explain Objectives & $0.35 *$ & $0.38 *$ \\
\hline $\mathrm{C}$ & Explain CAI & $0.57 *$ & $0.41 *$ \\
\hline $\mathrm{D}$ & Motivate & $0.31 *$ & $0.46 *$ \\
\hline E & Integrate & $0.62 *$ & $0.51 *$ \\
\hline $\mathrm{F}$ & Individual & $0.34 *$ & 0.28 \\
\hline $\mathrm{G}$ & Small Group & $0.38 *$ & $0.48 *$ \\
\hline $\mathrm{H}$ & Whole Class & $0.49 *$ & $0.68 *$ \\
\hline I & Technical Support & 0.09 & $0.39 *$ \\
\hline $\mathrm{J}$ & Instructional Support & 0.26 & 0.22 \\
\hline $\mathrm{K}$ & Post Instruction & 0.27 & 0.12 \\
\hline $\mathrm{L}$ & Follow-up & 0.21 & $0.41 *$ \\
\hline M & Required & $0.35 *$ & 0.18 \\
\hline $\mathrm{N}$ & Optional & $0.57 *$ & $0.56 *$ \\
\hline $\mathrm{O}$ & Course Exam & $0.50 *$ & $0.46 *$ \\
\hline $\mathrm{P}$ & Embedded & $0.51 *$ & $0.50 *$ \\
\hline Q & Replace Traditional & 0.18 & $0.60 *$ \\
\hline $\mathrm{R}$ & Alternative & $0.46 *$ & $0.52 *$ \\
\hline S & Evaluate CAI & $0.31 *$ & $0.46 *$ \\
\hline
\end{tabular}

* Significant at $\mathrm{p}<0.01$ 
Table C 27. Comparison of Correlations Between Frequency of Use and Perceived Effectiveness of Integration Strategies for Increasing Student Learning by Type of Institution $(\mathrm{n}=77)$

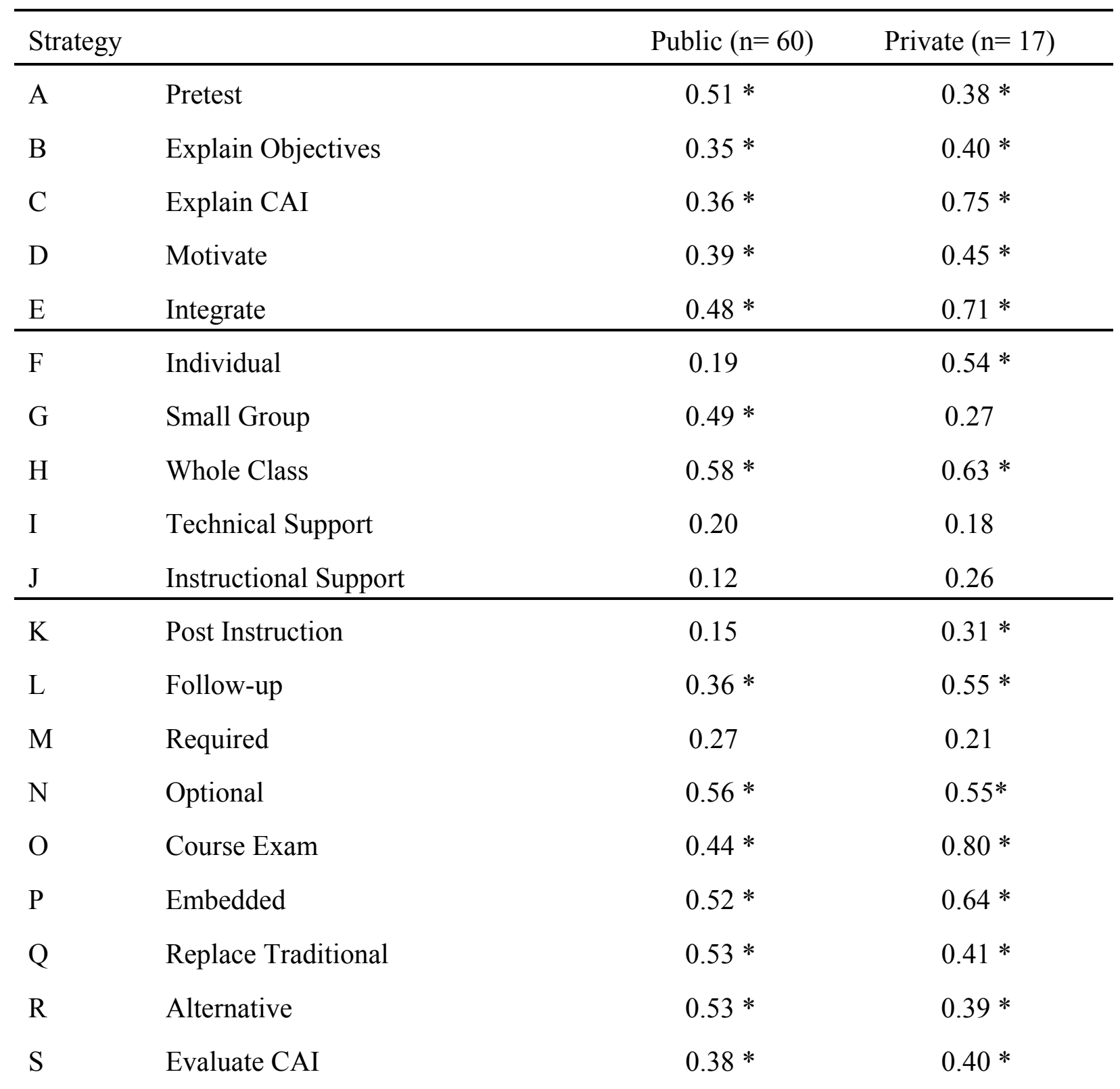

* Significant at $\mathrm{p}<0.01$ 
Table C 28. Comparison of Correlations Between Frequency of Use and Perceived Effectiveness of Integration Strategies for Increasing Student Learning by School Size $\quad(n=77)$

\begin{tabular}{|c|c|c|c|c|}
\hline Strategy & & Small $(n=25)$ & Medium $(n=28)$ & Large $(n=24)$ \\
\hline A & Pretest & $0.51 *$ & $0.51 *$ & $0.55 *$ \\
\hline $\mathrm{B}$ & Explain Objectives & $0.61 *$ & -0.24 & $0.48 *$ \\
\hline $\mathrm{C}$ & Explain CAI & $0.61 *$ & -0.08 & $0.33 *$ \\
\hline $\mathrm{D}$ & Motivate & $0.66 *$ & 0.10 & $0.40 *$ \\
\hline $\mathrm{E}$ & Integrate & $0.50 *$ & 0.07 & $0.72 *$ \\
\hline $\mathrm{F}$ & Individual & $0.67 *$ & $0.38 *$ & -0.15 \\
\hline G & Small Group & $0.55 *$ & $0.39 *$ & $0.41 *$ \\
\hline $\mathrm{H}$ & Whole Class & $0.48 *$ & 0.28 & $0.75 *$ \\
\hline I & Technical Support & $0.39 *$ & 0.23 & 0.02 \\
\hline $\mathrm{J}$ & Instructional Support & $0.49 *$ & 0.06 & 0.15 \\
\hline $\mathrm{K}$ & Post Instruction & $0.36 *$ & -0.16 & $0.49 *$ \\
\hline $\mathrm{L}$ & Follow-up & $0.48 *$ & 0.16 & $0.51 *$ \\
\hline M & Required & $0.39 *$ & 0.24 & 0.29 \\
\hline $\mathrm{N}$ & Optional & $0.60 *$ & $0.50 *$ & $0.64 *$ \\
\hline $\mathrm{O}$ & Course Exam & $0.50 *$ & $0.31 *$ & $0.61 *$ \\
\hline $\mathrm{P}$ & Embedded & $0.68 *$ & $0.46 *$ & $0.44 *$ \\
\hline Q & Replace Traditional & $0.44 *$ & $0.47 *$ & $0.55 *$ \\
\hline $\mathrm{R}$ & Alternative & $0.52 *$ & $0.36 *$ & $0.60 *$ \\
\hline S & Evaluate CAI & $0.38 *$ & 0.14 & $0.53 *$ \\
\hline
\end{tabular}

* Significant at $\mathrm{p}<0.01$ 
Table C 29. Correlation Between Frequency of Use and Perceived Effectiveness of Strategies to Decrease Costs by Type of School $(\mathrm{N}=77)$

\begin{tabular}{llll}
\hline Strategy & & BSN & AD \\
\hline F & Individual & $0.38^{*}$ & 0.03 \\
$\mathrm{G}$ & Small Group & $0.41^{*}$ & -0.06 \\
$\mathrm{H}$ & Whole Class & $0.55^{*}$ & 0.15 \\
$\mathrm{I}$ & Technical Support & -0.01 & -0.15 \\
$\mathrm{~J}$ & Instructional Support & 0.12 & -0.11 \\
\hline $\mathrm{M}$ & Required & $0.40^{*}$ & 0.19 \\
$\mathrm{~N}$ & Optional & -0.20 & 0.13 \\
$\mathrm{Q}$ & Replace Traditional & 0.22 & 0.05 \\
$*$ Significant at $\mathrm{p}<0.01$ & &
\end{tabular}


Table C 30. Correlation Between Frequency of Use and Perceived Effectiveness of Strategies to Decrease Costs by Type of Institution $(\mathrm{N}=77)$

\begin{tabular}{llcc}
\hline Strategy & & Public & Private \\
\hline F & Individual & $0.33^{*}$ & $0.30^{*}$ \\
G & Small Group & 0.05 & 0.26 \\
H & Whole Class & $0.33^{*}$ & 0.10 \\
I & Technical Support & -0.20 & 0.16 \\
J & Instructional Support & -0.19 & 0.29 \\
\hline M & Required & 0.23 & $0.61^{*}$ \\
N & Optional & 0.11 & -0.24 \\
Q & Replace Traditional & 0.10 & $0.41^{*}$ \\
$*$ Significant at $p<0.01$ & &
\end{tabular}


Table C 31. Correlation Between Frequency of Use and Perceived Effectiveness of Strategies to Decrease Costs by School Size $(\mathrm{N}=77)$

\begin{tabular}{llccc}
\hline Strategy & & Small & Medium & Large \\
\hline $\mathrm{F}$ & Individual & 0.18 & 0.13 & 0.18 \\
$\mathrm{G}$ & Small Group & 0.19 & 0.03 & 0.28 \\
$\mathrm{H}$ & Whole Class & $0.52^{*}$ & -0.05 & $0.68^{*}$ \\
$\mathrm{I}$ & Technical Support & 0.03 & -0.20 & -0.01 \\
$\mathrm{~J}$ & Instructional Support & 0.24 & -0.19 & 0.03 \\
\hline $\mathrm{M}$ & Required & $0.53^{*}$ & $0.30^{*}$ & 0.10 \\
$\mathrm{~N}$ & Optional & -0.03 & -0.11 & $0.43^{*}$ \\
$\mathrm{Q}$ & Replace Traditional & $0.38^{*}$ & 0.26 & $0.50^{*}$ \\
$*$ Significant at $\mathrm{p}<0.01$ & & &
\end{tabular}


Table C 32. Correlation Between Frequency of Use and Perceived Effectiveness of Strategies to Decrease the Students' Time by Type of School $(\mathrm{N}=77)$

\begin{tabular}{llcc}
\hline Strategy & & BSN & AD \\
\hline B & Explain Objectives & 0.14 & 0.31 \\
$\mathrm{C}$ & Explain CAI & $0.57^{*}$ & 0.14 \\
\hline $\mathrm{F}$ & Individual & 0.01 & 0.14 \\
$\mathrm{G}$ & Small Group & 0.11 & $0.45^{*}$ \\
$\mathrm{H}$ & Whole Class & 0.17 & $0.38^{*}$ \\
$\mathrm{I}$ & Instructional Support & -0.03 & -0.16 \\
$\mathrm{~J}$ & Technical Support & 0.15 & -0.11 \\
& & & \\
* Significant at $\mathrm{p}<0.01$ & &
\end{tabular}


Table C 33. Correlation Between Frequency of Use and Perceived Effectiveness of Strategies to Decrease the Students' Time by Type of Institution $(\mathrm{N}=77)$

\begin{tabular}{llcc}
\hline Strategy & & Public & Private \\
\hline B & Explain Objectives & 0.22 & 0.28 \\
$\mathrm{C}$ & Explain CAI & 0.12 & $0.75^{*}$ \\
\hline $\mathrm{F}$ & Individual & 0.08 & 0.12 \\
$\mathrm{G}$ & Small Group & $0.32^{*}$ & $0.38^{*}$ \\
$\mathrm{H}$ & Whole Class & 0.37 & 0.09 \\
$\mathrm{I}$ & Instructional Support & -0.14 & -0.11 \\
$\mathrm{~J}$ & Technical Support & -0.15 & 0.21 \\
$*$ Significant at $\mathrm{p}<0.01$ & &
\end{tabular}


Table C 34. Correlation Between Frequency of Use and Perceived Effectiveness of Strategies to Decrease the Students' Time by School Size $(\mathrm{N}=77)$

\begin{tabular}{llccc}
\hline Strategy & & Small & Medium & Large \\
\hline B & Explain Objectives & $0.45^{*}$ & -0.14 & 0.15 \\
C & Explain CAI & $0.51^{*}$ & -0.11 & 0.18 \\
\hline F & Individual & 0.04 & -0.15 & 0.22 \\
G & Small Group & $0.39^{*}$ & 0.09 & $0.31^{*}$ \\
H & Whole Class & $0.43^{*}$ & 0.04 & -0.07 \\
I & Instructional Support & 0.27 & -0.26 & -0.09 \\
J & Technical Support & -0.14 & $-0.46^{*}$ & -0.01
\end{tabular}

* Significant at $\mathrm{p}<0.01$ 
Table C 35. Correlation Between Frequency of Use and Perceived Effectiveness of Strategies to Decrease the Teachers' Time by Type of School $(\mathrm{N}=77)$

\begin{tabular}{llll}
\hline Strategy & & BSN & AD \\
\hline F & Individual & $-0.31^{*}$ & 0.07 \\
$\mathrm{G}$ & Small Group & -0.01 & 0.22 \\
$\mathrm{H}$ & Whole Class & -0.00 & 0.32 \\
$\mathrm{I}$ & Technical Support & 0.08 & 0.03 \\
$\mathrm{~J}$ & Instructional Support & 0.11 & 0.01 \\
\hline $\mathrm{M}$ & Required & -0.06 & 0.08 \\
$\mathrm{~N}$ & Optional & $0.46^{*}$ & 0.43 \\
$\mathrm{O}$ & Course Exam & 0.14 & 0.13 \\
$\mathrm{P}$ & Embedded & 0.29 & 0.08 \\
$\mathrm{Q}$ & Replace Traditional & 0.34 & 0.07
\end{tabular}

* Significant at $\mathrm{p}<0.01$ 
Table C 36. Correlation Between Frequency of Use and Perceived Effectiveness of Strategies to Decrease the Teachers' Time by Type of Institution $(\mathrm{N}=77)$

\begin{tabular}{llcc}
\hline Strategy & & Public & Private \\
\hline F & Individual & -0.12 & -0.11 \\
$\mathrm{G}$ & Small Group & 0.11 & 0.29 \\
$\mathrm{H}$ & Whole Class & 0.25 & -0.10 \\
$\mathrm{I}$ & Technical Support & -0.00 & 0.01 \\
$\mathrm{~J}$ & Instructional Support & -0.06 & 0.21 \\
\hline $\mathrm{M}$ & Required & -0.07 & $0.52^{*}$ \\
$\mathrm{~N}$ & Optional & $0.52^{*}$ & -0.03 \\
$\mathrm{O}$ & Course Exam & -0.03 & $0.74^{*}$ \\
$\mathrm{P}$ & Embedded & 0.14 & $0.44^{*}$ \\
$\mathrm{Q}$ & Replace Traditional & 0.13 & 0.23
\end{tabular}

* Significant at $\mathrm{p}<0.01$ 
Table C 37. Correlation Between Frequency of Use and Perceived Effectiveness of Strategies to Decrease the Teachers' Time by School Size $(\mathrm{N}=77)$

\begin{tabular}{llccc}
\hline Strategy & & Small & Medium & Large \\
\hline $\mathrm{F}$ & Individual & 0.02 & 0.04 & $-0.49^{*}$ \\
$\mathrm{G}$ & Small Group & 0.22 & 0.05 & 0.15 \\
$\mathrm{H}$ & Whole Class & 0.25 & -0.05 & $0.32^{*}$ \\
$\mathrm{I}$ & Technical Support & 0.00 & 0.11 & -0.16 \\
$\mathrm{~J}$ & Instructional Support & -0.05 & 0.01 & 0.03 \\
\hline $\mathrm{M}$ & Required & $0.38^{*}$ & 0.13 & $-0.48^{*}$ \\
$\mathrm{~N}$ & Optional & 0.28 & $0.53 *$ & $0.57^{*}$ \\
$\mathrm{O}$ & Course Exam & $0.38^{*}$ & 0.13 & -0.27 \\
$\mathrm{P}$ & Embedded & $0.42^{*}$ & $0.31 *$ & -0.16 \\
$\mathrm{Q}$ & Replace Traditional & 0.28 & 0.04 & 0.16
\end{tabular}

* Significant at $\mathrm{p}<0.01$ 
Table C 38. Schools with a Computer Lab in the School of Nursing $(N=109)$

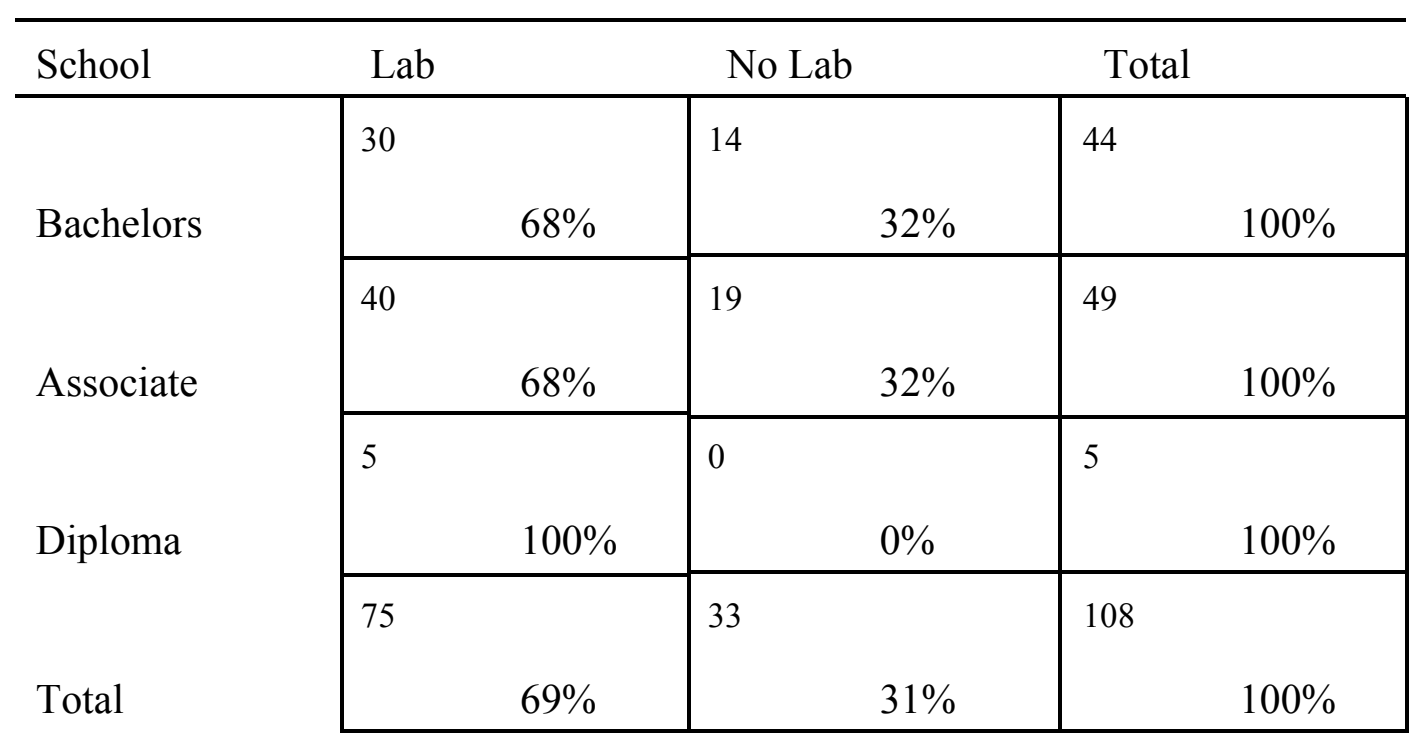

No Response $=1$ 
Table C 39. Institutions with a Computer Lab in the School of Nursing $(\mathrm{N}=109)$

\begin{tabular}{|c|c|c|c|}
\hline Institution & $\mathrm{Lab}$ & No Lab & Total \\
\hline \multirow{3}{*}{ Public } & 59 & 24 & 83 \\
\hline & $71 \%$ & $29 \%$ & $100 \%$ \\
\hline & 16 & 9 & 25 \\
\hline \multirow[t]{2}{*}{ Private } & $64 \%$ & $36 \%$ & $100 \%$ \\
\hline & 75 & 33 & 108 \\
\hline Total & $69 \%$ & $31 \%$ & $100 \%$ \\
\hline
\end{tabular}

No Response $=1$ 
Table C 40. Schools with a Computer Lab in the School of Nursing based on School Size $(\mathrm{N}=109)$

\begin{tabular}{|c|c|c|c|}
\hline Institution & $\mathrm{Lab}$ & No Lab & Total \\
\hline \multirow{3}{*}{ Small } & 25 & 12 & 37 \\
\hline & $68 \%$ & $32 \%$ & $100 \%$ \\
\hline & 25 & 17 & 29 \\
\hline \multirow[t]{2}{*}{ Medium } & $60 \%$ & $40 \%$ & $100 \%$ \\
\hline & 24 & 5 & 29 \\
\hline \multirow[t]{2}{*}{ Large } & $83 \%$ & $17 \%$ & $100 \%$ \\
\hline & 74 & 34 & 108 \\
\hline Total & $69 \%$ & $31 \%$ & $100 \%$ \\
\hline
\end{tabular}

No Response $=1$ 
Table C 41. Number and Percent of Computers in Four Locations Reported by CAI-user and

Non-user Schools $(\mathrm{N}=109)$

\begin{tabular}{|c|c|c|c|c|c|}
\hline School & $\begin{array}{l}\text { School } \\
\text { of Nursing }\end{array}$ & $\begin{array}{l}\text { Another } \\
\text { Department }\end{array}$ & Library & $\begin{array}{l}\text { Students } \\
\text { Purchase }\end{array}$ & Total \\
\hline \multirow{3}{*}{ CAI-users } & 65 & 48 & 63 & 43 & 219 \\
\hline & $30 \%$ & $22 \%$ & $29 \%$ & $20 \%$ & $100 \%$ \\
\hline & 23 & 15 & 26 & 20 & 84 \\
\hline \multirow[t]{2}{*}{ Non-users } & $26 \%$ & $18 \%$ & $31 \%$ & $24 \%$ & $100 \%$ \\
\hline & 88 & 63 & 89 & 63 & 303 \\
\hline Totals & $29 \%$ & $21 \%$ & $29 \%$ & $21 \%$ & $100 \%$ \\
\hline
\end{tabular}


Table C 42. CAI-users and Non-user Schools Reporting Computers in Four Locations ( $N=109)$

\begin{tabular}{|c|c|c|c|c|c|}
\hline School & $\begin{array}{l}\text { School } \\
\text { of Nursing }\end{array}$ & $\begin{array}{l}\text { Another } \\
\text { Department }\end{array}$ & Library & $\begin{array}{l}\text { Students } \\
\text { Purchase }\end{array}$ & Total \\
\hline & 65 & 48 & 63 & 43 & 219 \\
\hline \multirow[t]{2}{*}{ CAI-users } & $74 \%$ & $76 \%$ & $71 \%$ & $68 \%$ & $72 \%$ \\
\hline & 23 & 15 & 26 & 20 & 84 \\
\hline \multirow[t]{2}{*}{ Non-Users } & $26 \%$ & $24 \%$ & $29 \%$ & $32 \%$ & $28 \%$ \\
\hline & 88 & 63 & 89 & 63 & 303 \\
\hline Totals & $100 \%$ & $100 \%$ & $100 \%$ & $100 \%$ & $100 \%$ \\
\hline
\end{tabular}


Table C 43. Types of Schools Reporting Computers in Four Locations ( $\mathrm{N}=109)$

\begin{tabular}{|c|c|c|c|c|c|c|}
\hline School & $\begin{array}{l}\text { School } \\
\text { of Nursing }\end{array}$ & $\begin{array}{l}\text { Another } \\
\text { Department }\end{array}$ & Library & $\begin{array}{l}\text { Students } \\
\text { Purchase }\end{array}$ & Total & $\begin{array}{l}\text { Percent } \\
\text { of All }\end{array}$ \\
\hline \multirow{3}{*}{ Bachelors } & 38 & 25 & 39 & 34 & 136 & $45 \%$ \\
\hline & $28 \%$ & $18 \%$ & $29 \%$ & $25 \%$ & \multicolumn{2}{|l|}{$100 \%$} \\
\hline & 46 & 37 & 46 & 28 & 157 & $52 \%$ \\
\hline \multirow[t]{2}{*}{ Associate } & $29 \%$ & $24 \%$ & $29 \%$ & $18 \%$ & \multicolumn{2}{|l|}{$100 \%$} \\
\hline & 4 & 1 & 4 & 1 & 10 & $3 \%$ \\
\hline \multirow[t]{2}{*}{ Diploma } & $40 \%$ & $10 \%$ & $40 \%$ & $10 \%$ & $100 \%$ & \\
\hline & 88 & 63 & 89 & 63 & 303 & $100 \%$ \\
\hline Total & $29 \%$ & $21 \%$ & $29 \%$ & $21 \%$ & $100 \%$ & \\
\hline
\end{tabular}


Table C 44. Institutions Reporting Computers in Four Locations (N=109)

\begin{tabular}{|c|c|c|c|c|c|c|}
\hline School & $\begin{array}{l}\text { School } \\
\text { of Nursing }\end{array}$ & $\begin{array}{l}\text { Another } \\
\text { Department }\end{array}$ & Library & $\begin{array}{l}\text { Students } \\
\text { Purchase }\end{array}$ & Total & $\begin{array}{l}\text { Percent } \\
\text { of All }\end{array}$ \\
\hline & 70 & 51 & 67 & 44 & 232 & $77 \%$ \\
\hline \multirow[t]{2}{*}{ Public } & $30 \%$ & $22 \%$ & $29 \%$ & $19 \%$ & $100 \%$ & \\
\hline & 18 & 12 & 22 & 19 & 71 & $23 \%$ \\
\hline \multirow[t]{2}{*}{ Private } & $25 \%$ & $17 \%$ & $31 \%$ & $27 \%$ & $100 \%$ & \\
\hline & 88 & 63 & 89 & 63 & 303 & $100 \%$ \\
\hline Total & $29 \%$ & $21 \%$ & $29 \%$ & $21 \%$ & $100 \%$ & \\
\hline
\end{tabular}


Table C 45. School Sizes Reporting Computers in Four Locations ( $N=109)$

\begin{tabular}{|c|c|c|c|c|c|c|}
\hline School & $\begin{array}{l}\text { School } \\
\text { of Nursing }\end{array}$ & $\begin{array}{l}\text { Another } \\
\text { Department }\end{array}$ & Library & $\begin{array}{l}\text { Students } \\
\text { Purchase }\end{array}$ & Total & $\begin{array}{l}\text { Percent } \\
\text { of All }\end{array}$ \\
\hline \multirow{3}{*}{ Small } & 29 & 25 & 30 & 17 & 101 & $33 \%$ \\
\hline & $29 \%$ & $25 \%$ & $30 \%$ & $17 \%$ & \multicolumn{2}{|l|}{$100 \%$} \\
\hline & 32 & 23 & 35 & 27 & 117 & $39 \%$ \\
\hline \multirow[t]{2}{*}{ Medium } & $27 \%$ & $20 \%$ & $30 \%$ & $23 \%$ & \multicolumn{2}{|l|}{$100 \%$} \\
\hline & 27 & 15 & 24 & 19 & 85 & $28 \%$ \\
\hline \multirow[t]{2}{*}{ Large } & $32 \%$ & $18 \%$ & $28 \%$ & $22 \%$ & \multicolumn{2}{|l|}{$100 \%$} \\
\hline & 88 & 63 & 89 & 63 & 303 & $100 \%$ \\
\hline Total & $29 \%$ & $21 \%$ & $29 \%$ & $21 \%$ & $100 \%$ & \\
\hline
\end{tabular}


Table C 46. Percent of Computers in Four Locations Owned by CAI-user and Non-user Schools $(\mathrm{N}=109)$

\begin{tabular}{|c|c|c|c|c|c|}
\hline School & $\begin{array}{l}\text { School } \\
\text { of Nursing }\end{array}$ & $\begin{array}{l}\text { Another } \\
\text { Department }\end{array}$ & Library & Total & \\
\hline \multirow{3}{*}{ CAI-users } & 1,317 & 4,032 & 3,041 & 8,390 & \multirow[b]{2}{*}{$68 \%$} \\
\hline & $76 \%$ & $65 \%$ & $79 \%$ & & \\
\hline & 419 & 2,124 & 1,332 & 3,875 & \\
\hline \multirow[t]{2}{*}{ Non-Users } & $24 \%$ & $35 \%$ & $30 \%$ & & $32 \%$ \\
\hline & 1,736 & 6,156 & 4,394 & 12,265 & \\
\hline Total & $100 \%$ & $100 \%$ & $100 \%$ & & $100 \%$ \\
\hline
\end{tabular}


Table C 47. Number of Computers in Four Locations Owned by CAI-user and Non-user Schools $(\mathrm{N}=109)$

\begin{tabular}{|c|c|c|c|c|c|}
\hline School & $\begin{array}{l}\text { School } \\
\text { of Nursing }\end{array}$ & $\begin{array}{l}\text { Another } \\
\text { Department }\end{array}$ & Library & Total & \\
\hline \multirow{3}{*}{ CAI-users } & 1,317 & 4,032 & 3,041 & 8,390 & \multirow[b]{2}{*}{$100 \%$} \\
\hline & $16 / \%$ & $48 \%$ & $36 \%$ & & \\
\hline & 419 & 2,124 & 1,332 & 3,875 & \\
\hline \multirow[t]{2}{*}{ Non-Users } & $11 \%$ & $55 \%$ & $34 \%$ & & $100 \%$ \\
\hline & 1,736 & 6,156 & 4,394 & 12,265 & \\
\hline Total & $14 \%$ & $50 \%$ & $36 \%$ & & $100 \%$ \\
\hline
\end{tabular}


Table C 48. Number of Computers in Four Locations by Type of School $(\mathrm{N}=109)$

\begin{tabular}{|c|c|c|c|c|c|}
\hline School & $\begin{array}{l}\text { School } \\
\text { of Nursing }\end{array}$ & $\begin{array}{l}\text { Another } \\
\text { Department }\end{array}$ & Library & Total & Percen \\
\hline \multirow{3}{*}{ Bachelors } & 760 & 3,000 & 2,950 & 6,710 & \\
\hline & $11 \%$ & $45 \%$ & $44 \%$ & $100 \%$ & $26 \%$ \\
\hline & 935 & 3,116 & 1,390 & 5,441 & \\
\hline \multirow[t]{2}{*}{ Associate } & $17 \%$ & $57 \%$ & $26 \%$ & $100 \%$ & $46 \%$ \\
\hline & 41 & 40 & 33 & 114 & \\
\hline \multirow[t]{2}{*}{ Diploma } & $36 \%$ & $35 \%$ & $29 \%$ & $100 \%$ & $29 \%$ \\
\hline & 1,738 & 6,156 & 4,373 & 12,265 & \\
\hline Total & $14 \%$ & $50 \%$ & $36 \%$ & $100 \%$ & $100 \%$ \\
\hline
\end{tabular}


Table C 49. Number of Computers in Four Locations by Type of Institution $(\mathrm{N}=109)$

\begin{tabular}{|c|c|c|c|c|c|}
\hline School & $\begin{array}{l}\text { School } \\
\text { of Nursing }\end{array}$ & $\begin{array}{l}\text { Another } \\
\text { Department }\end{array}$ & Library & Total & Percent \\
\hline & 1,453 & 5,391 & 3,863 & 10,707 & \\
\hline \multirow[t]{2}{*}{ Public } & $14 \%$ & $50 \%$ & $36 \%$ & $100 \%$ & $87 \%$ \\
\hline & 283 & 765 & 510 & 1,558 & \\
\hline \multirow[t]{2}{*}{ Private } & $18 \%$ & $49 \%$ & $33 \%$ & $100 \%$ & $13 \%$ \\
\hline & 1,736 & 6,156 & 4,373 & 12,265 & \\
\hline Total & $14 \%$ & $50 \%$ & $36 \%$ & $100 \%$ & $100 \%$ \\
\hline
\end{tabular}


Table C 50. Number of Computers in Four Locations by School Size (N=109)

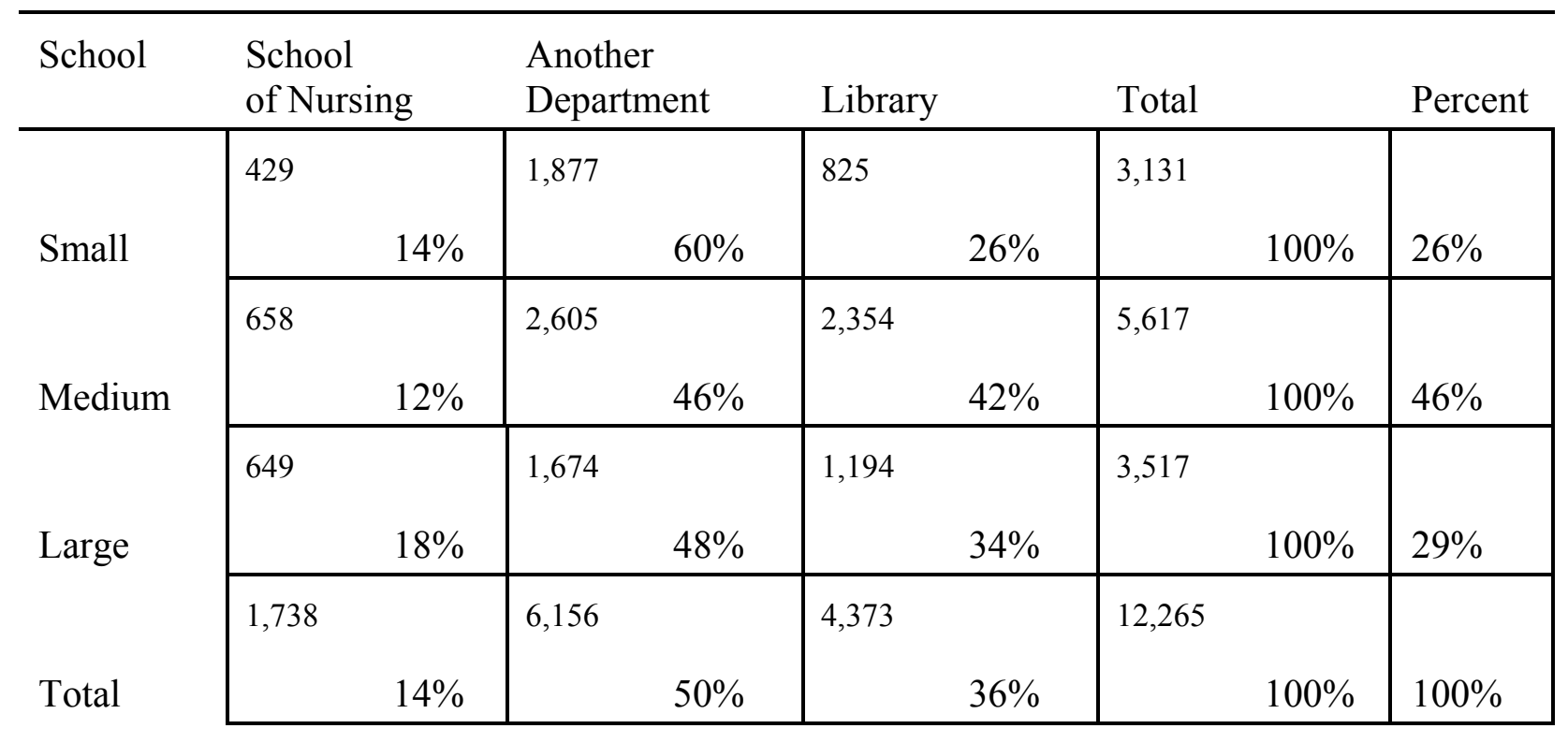


Table C 51. Full and Part-time Technical and Instructional Support Personal Employed by CAI-user and Non-user Schools $(\mathrm{N}=109)$

Technical Support Personnel

\begin{tabular}{|c|c|c|c|}
\hline & Full-time & Part-time & Total \\
\hline \multirow{3}{*}{ CAI-users } & 240 & 160 & 400 \\
\hline & $86 \%$ & $84 \%$ & $85 \%$ \\
\hline & 38 & 31 & 69 \\
\hline \multirow[t]{2}{*}{ Non-users } & $14 \%$ & $16 \%$ & $15 \%$ \\
\hline & 278 & 191 & 469 \\
\hline Total & $100 \%$ & $100 \%$ & $100 \%$ \\
\hline
\end{tabular}

Instructional Support Personnel

\begin{tabular}{|c|c|c|c|}
\hline & Full-time & Part-time & Total \\
\hline \multirow{3}{*}{ CAI-users } & 143 & 73 & 216 \\
\hline & $91 \%$ & $88 \%$ & $90 \%$ \\
\hline & 15 & 10 & 25 \\
\hline \multirow[t]{2}{*}{ Non-users } & $9 \%$ & $12 \%$ & $10 \%$ \\
\hline & 158 & 83 & 241 \\
\hline Total & $100 \%$ & $100 \%$ & $100 \%$ \\
\hline
\end{tabular}


Table C 52. Comparison of the Percent of Full and Part-time Technical and Instructional Support Personal Employed by CAI-user and Non-user Schools $(\mathrm{N}=109)$

Technical Support Personnel

\begin{tabular}{|c|c|c|c|}
\hline & Full-time & Part-time & Total \\
\hline \multirow{3}{*}{ CAI-users } & 240 & 160 & 400 \\
\hline & $60 \%$ & $40 \%$ & $100 \%$ \\
\hline & 38 & 31 & 69 \\
\hline \multirow[t]{2}{*}{ Non-users } & $55 \%$ & $45 \%$ & $100 \%$ \\
\hline & 278 & 191 & 469 \\
\hline Total & $59 \%$ & $41 \%$ & $100 \%$ \\
\hline
\end{tabular}

Instructional Support Personnel

\begin{tabular}{|c|c|c|c|}
\hline & Full-time & Part-time & Total \\
\hline \multirow{3}{*}{ CAI-users } & 143 & 73 & 216 \\
\hline & $66 \%$ & $34 \%$ & $100 \%$ \\
\hline & 15 & 10 & 24 \\
\hline \multirow[t]{2}{*}{ Non-users } & $60 \%$ & $40 \%$ & $100 \%$ \\
\hline & 158 & 83 & 241 \\
\hline Total & $66 \%$ & $34 \%$ & $100 \%$ \\
\hline
\end{tabular}


Table C 53. Full and Part-time Technical and Instructional Support Personal Employed by Three Types of Schools $(\mathrm{N}=109)$

Technical Support Personnel

\begin{tabular}{|c|c|c|c|}
\hline & Full-time & Part-time & Total \\
\hline \multirow{3}{*}{ Bachelors } & 104 & 69 & 173 \\
\hline & $37 \%$ & $36 \%$ & $37 \%$ \\
\hline & 172 & 120 & 292 \\
\hline \multirow[t]{2}{*}{ Associate } & $62 \%$ & $63 \%$ & $62 \%$ \\
\hline & 2 & 2 & 4 \\
\hline \multirow[t]{2}{*}{ Diploma } & $1 \%$ & $1 \%$ & $1 \%$ \\
\hline & 278 & 191 & 469 \\
\hline Total & $100 \%$ & $100 \%$ & $100 \%$ \\
\hline
\end{tabular}

Instructional Support Personnel

\begin{tabular}{|c|c|c|c|}
\hline & Full-time & Part-time & Total \\
\hline \multirow{3}{*}{ Bachelors } & 69 & 34 & 103 \\
\hline & $44 \%$ & $41 \%$ & $43 \%$ \\
\hline & 89 & 49 & 138 \\
\hline \multirow[t]{2}{*}{ Associate } & $56 \%$ & $59 \%$ & $59 \%$ \\
\hline & 0 & 0 & 0 \\
\hline \multirow[t]{2}{*}{ Diploma } & $0 \%$ & $0 \%$ & $0 \%$ \\
\hline & 158 & 83 & 241 \\
\hline Total & $100 \%$ & $100 \%$ & $100 \%$ \\
\hline
\end{tabular}


Table C 54. Comparison of the Percent of Full and Part-time Technical and Instructional Support Personal Employed by Three Types of Schools $(\mathrm{N}=109)$

Technical Support Personnel

\begin{tabular}{|c|c|c|c|}
\hline & Full-time & Part-time & Total \\
\hline \multirow{3}{*}{ Bachelors } & 104 & 69 & 173 \\
\hline & $60 \%$ & $40 \%$ & $100 \%$ \\
\hline & 172 & 120 & 292 \\
\hline \multirow[t]{2}{*}{ Associate } & $59 \%$ & $41 \%$ & $100 \%$ \\
\hline & 2 & 2 & 4 \\
\hline \multirow[t]{2}{*}{ Diploma } & $50 \%$ & $50 \%$ & $100 \%$ \\
\hline & 278 & 191 & 469 \\
\hline Total & $59 \%$ & $41 \%$ & $100 \%$ \\
\hline
\end{tabular}

Instructional Support Personnel

\begin{tabular}{|c|c|c|c|}
\hline & Full-time & Part-time & Total \\
\hline \multirow{3}{*}{ Bachelors } & 69 & 34 & 103 \\
\hline & $67 \%$ & $33 \%$ & $100 \%$ \\
\hline & 89 & 49 & 138 \\
\hline \multirow[t]{2}{*}{ Associate } & $64 \%$ & $36 \%$ & $100 \%$ \\
\hline & 0 & 0 & 0 \\
\hline \multirow[t]{2}{*}{ Diploma } & $0 \%$ & $0 \%$ & $100 \%$ \\
\hline & 158 & 83 & 241 \\
\hline Total & $66 \%$ & $34 \%$ & $100 \%$ \\
\hline
\end{tabular}


Table C 55. Full and Part-time Technical and Instructional Support Personal Employed by Types of Institutions $(\mathrm{N}=109)$

Technical Support Personnel

\begin{tabular}{|c|c|c|c|}
\hline & Full-time & Part-time & Total \\
\hline \multirow{3}{*}{ Public } & 233 & 160 & 393 \\
\hline & $84 \%$ & $84 \%$ & $84 \%$ \\
\hline & 45 & 31 & 76 \\
\hline \multirow[t]{2}{*}{ Private } & $16 \%$ & $16 \%$ & $16 \%$ \\
\hline & 278 & 191 & 469 \\
\hline Total & $100 \%$ & $100 \%$ & $100 \%$ \\
\hline
\end{tabular}

Instructional Support Personnel

\begin{tabular}{|c|c|c|c|}
\hline & Full-time & Part-time & Total \\
\hline \multirow{3}{*}{ Public } & 121 & 60 & 181 \\
\hline & $77 \%$ & $72 \%$ & $75 \%$ \\
\hline & 37 & 23 & 60 \\
\hline \multirow[t]{2}{*}{ Private } & $23 \%$ & $28 \%$ & $25 \%$ \\
\hline & 158 & 83 & 241 \\
\hline Total & $100 \%$ & $100 \%$ & $100 \%$ \\
\hline
\end{tabular}


Table C 56. Full and Part-time Technical and Instructional Support Personal by Employed by Different School Sizes $(\mathrm{N}=109)$

Technical Support Personnel

\begin{tabular}{|c|c|c|c|}
\hline & Full-time & Part-time & Total \\
\hline \multirow{3}{*}{ Small } & 82 & 82 & 164 \\
\hline & $29 \%$ & $43 \%$ & $35 \%$ \\
\hline & 92 & 44 & 136 \\
\hline \multirow[t]{2}{*}{ Medium } & $33 \%$ & $23 \%$ & $29 \%$ \\
\hline & 104 & 65 & 169 \\
\hline \multirow[t]{2}{*}{ Large } & $37 \%$ & $34 \%$ & $36 \%$ \\
\hline & 278 & 191 & 469 \\
\hline Total & $100 \%$ & $100 \%$ & $100 \%$ \\
\hline
\end{tabular}

Instructional Support Personnel

\begin{tabular}{|c|c|c|c|}
\hline & Full-time & Part-time & Total \\
\hline \multirow{3}{*}{ Small } & 45 & 19 & 64 \\
\hline & $28 \%$ & $23 \%$ & $27 \%$ \\
\hline & 61 & 29 & 90 \\
\hline \multirow[t]{2}{*}{ Medium } & $39 \%$ & $35 \%$ & $37 \%$ \\
\hline & 52 & 35 & 87 \\
\hline \multirow[t]{2}{*}{ Large } & $33 \%$ & $42 \%$ & $36 \%$ \\
\hline & 158 & 83 & 241 \\
\hline Total & $100 \%$ & $100 \%$ & $100 \%$ \\
\hline
\end{tabular}


Table C 57. Frequency Distribution and Polygon Graphic Illustration of the Percent of Faculty That Use CAI (N-109)

\begin{tabular}{lc}
\hline Percent of Faculty & Frequency \\
\hline $0 \%$ & 28 \\
1 to $10 \%$ & 6 \\
11 to $20 \%$ & 12 \\
21 to $30 \%$ & 10 \\
31 to $40 \%$ & 10 \\
41 to $50 \%$ & 12 \\
51 to $60 \%$ & 0 \\
61 to 70 & 1 \\
71 to 80 & 3 \\
81 to 90 & 5 \\
91 to 100 & 22 \\
Total & 109
\end{tabular}

Percent of Faculty That Use CAI

Frequency Distribution ( $\mathrm{N}=109)$

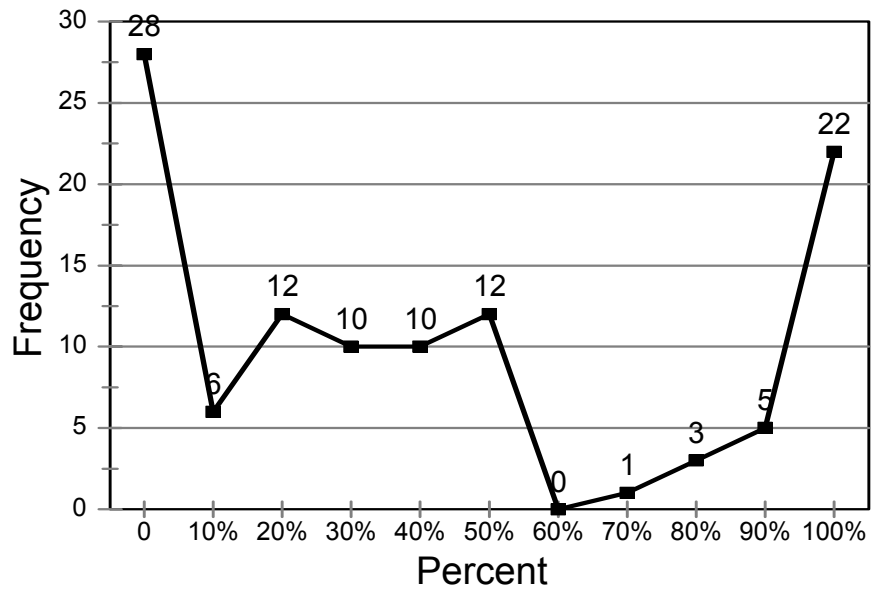


Table C 58. Frequency Distribution and Polygon Graphic Illustration of the Percent of Teachers That Use CAI by Type of School (N-109)

\section{Frequency}

\begin{tabular}{lcc}
\hline Percent of Teachers & BSN $(\mathrm{n}=45)$ & AD $(\mathrm{n}=59)$ \\
\hline $0 \%$ & 17 & 10 \\
1 to $10 \%$ & 4 & 1 \\
11 to $20 \%$ & 5 & 7 \\
21 to $30 \%$ & 4 & 5 \\
31 to $40 \%$ & 5 & 5 \\
41 to $50 \%$ & 7 & 4 \\
51 to $60 \%$ & 0 & 0 \\
61 to $70 \%$ & 0 & 1 \\
71 to $80 \%$ & 1 & 2 \\
81 to $90 \%$ & 0 & 5 \\
91 to $100 \%$ & 2 & 19
\end{tabular}

Percent of Faculty That Use CAI

Type of School

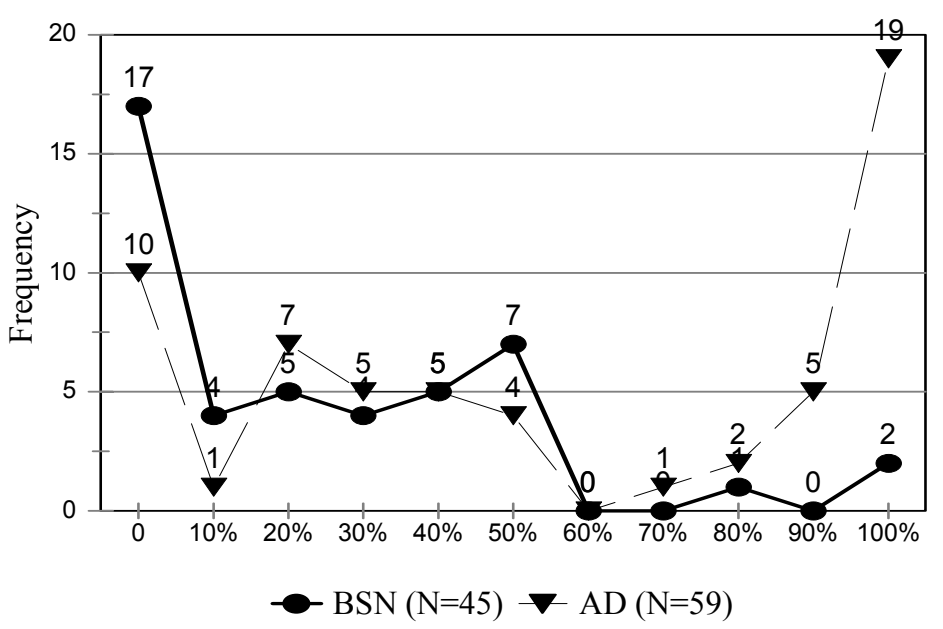


Table C 59. Frequency Distribution and Polygon Graphic Illustration of the Percent of Teachers That Use CAI by Type of Institution (N-109)

Frequency

\begin{tabular}{lcc}
\hline Percent of Faculty & Public $(\mathrm{n}=84)$ & Private $(\mathrm{n}=25)$ \\
\hline $0 \%$ & 22 & 6 \\
1 to $10 \%$ & 3 & 3 \\
11 to $20 \%$ & 9 & 3 \\
21 to $30 \%$ & 9 & 1 \\
31 to $40 \%$ & 7 & 3 \\
41 to $50 \%$ & 7 & 5 \\
51 to $60 \%$ & 0 & 0 \\
61 to $70 \%$ & 1 & 0 \\
71 to $80 \%$ & 1 & 2 \\
81 to $90 \%$ & 3 & 1 \\
91 to $100 \%$ & 21 & 1
\end{tabular}

Percent of Faculty That Use CAI

Type of Institution

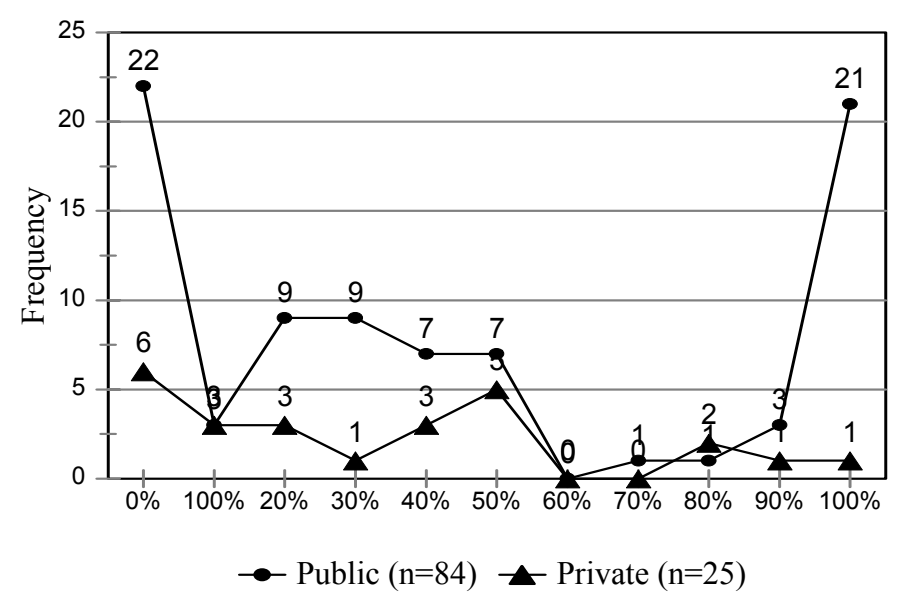


Table C 60. Frequency Distribution and Polygon Graphic Illustration of the Percent of Teachers That Use CAI by Type of Institution (N-109)

Frequency

\begin{tabular}{lrcc}
\hline Percent of Faculty & Small & Medium & Large \\
\hline $0 \%$ & 9 & 14 & 5 \\
1 to $10 \%$ & 2 & 2 & 2 \\
11 to $20 \%$ & 4 & 5 & 3 \\
21 to $30 \%$ & 3 & 6 & 1 \\
31 to $40 \%$ & 0 & 5 & 5 \\
41 to $50 \%$ & 1 & 5 & 6 \\
51 to $60 \%$ & 0 & 0 & 0 \\
61 to $70 \%$ & 1 & 0 & 0 \\
71 to $80 \%$ & 1 & 0 & 2 \\
81 to $90 \%$ & 2 & 1 & 0 \\
91 to $100 \%$ & 13 & 5 & 4
\end{tabular}

Percent of Faculty Use CAI

School Size

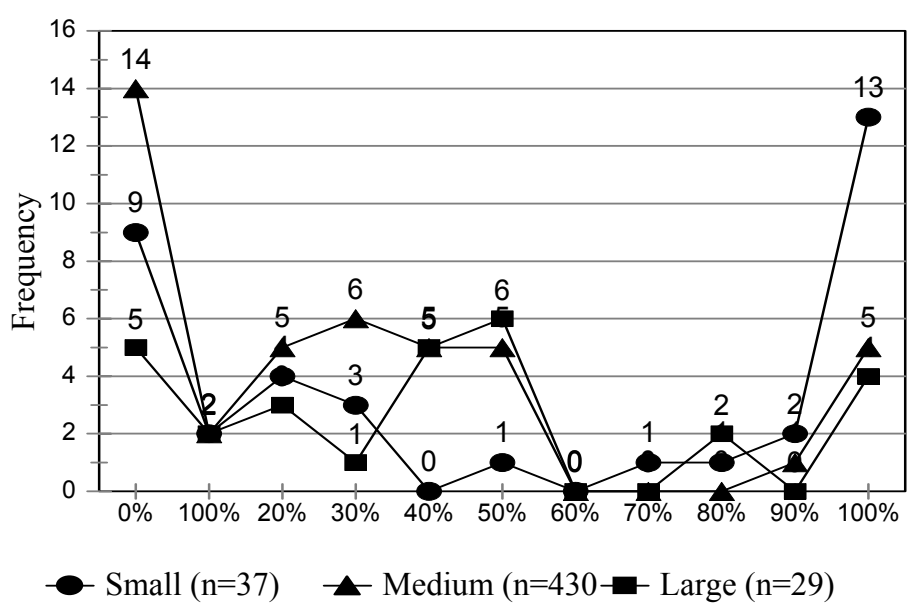


Table C 61. Types of Software Used by Sample Schools by Type of School (N=109)

\begin{tabular}{|c|c|c|c|c|c|c|}
\hline & Simulations & Tutorial & VR & ES & $\mathrm{D} \& \mathrm{P}$ & Total \\
\hline \multirow{3}{*}{ BSN } & 141 & 827 & 56 & 2 & 157 & 1,183 \\
\hline & $13 \%$ & $36 \%$ & $28 \%$ & $2 \%$ & $8 \%$ & $21 \%$ \\
\hline & 920 & 1,449 & 145 & 116 & 1,712 & 4,342 \\
\hline $\mathrm{AD}$ & $86 \%$ & $64 \%$ & $72 \%$ & $98 \%$ & $91 \%$ & $78 \%$ \\
\hline \multirow[t]{2}{*}{ Dip } & $0 \%$ & $0 \%$ & $0 \%$ & $0 \%$ & $0 \%$ & $0 \%$ \\
\hline & 1,065 & 2,278 & 201 & 118 & 1,878 & 5,540 \\
\hline Total & $100 \%$ & $100 \%$ & $100 \%$ & $100 \%$ & $100 \%$ & $100 \%$ \\
\hline
\end{tabular}

\begin{tabular}{|c|c|c|c|c|c|c|}
\hline & Simulations & Tutorial & VR & ES & D\&P & Total \\
\hline \multirow{3}{*}{ BSN } & 141 & 827 & 56 & 2 & 157 & 1,183 \\
\hline & $12 \%$ & $70 \%$ & $5 \%$ & $0 \%$ & $13 \%$ & $100 \%$ \\
\hline & 920 & 1,449 & 145 & 116 & 1,712 & 4,342 \\
\hline \multirow[t]{2}{*}{$\mathrm{AD}$} & $21 \%$ & $33 \%$ & $3 \%$ & $3 \%$ & $39 \%$ & $100 \%$ \\
\hline & 4 & 2 & 0 & 0 & 9 & 15 \\
\hline \multirow[t]{2}{*}{ Dip } & $27 \%$ & $13 \%$ & $0 \%$ & $0 \%$ & $60 \%$ & $100 \%$ \\
\hline & 1,065 & 2,278 & 201 & 118 & 1,878 & 5,540 \\
\hline Total & $19 \%$ & $41 \%$ & $4 \%$ & $2 \%$ & $34 \%$ & $100 \%$ \\
\hline
\end{tabular}


Table C 62 . Types of Software Used by Sample Schools based on Type of Institution (N=109)

\begin{tabular}{|c|c|c|c|c|c|c|}
\hline & Simulations & Tutorial & VR & ES & $\mathrm{D} \& \mathrm{P}$ & Total \\
\hline \multirow{3}{*}{ Public } & 1,003 & 2,135 & 183 & 116 & 1,795 & 5,232 \\
\hline & $94 \%$ & $94 \%$ & $91 \%$ & $98 \%$ & $94 \%$ & $94 \%$ \\
\hline & 62 & 143 & 18 & 2 & 83 & 308 \\
\hline \multirow[t]{2}{*}{ Private } & $6 \%$ & $6 \%$ & $9 \%$ & $3 \%$ & $3 \%$ & $6 \%$ \\
\hline & 1,065 & 2,278 & 201 & 118 & 1,878 & 5,540 \\
\hline Total & $100 \%$ & $100 \%$ & $100 \%$ & $100 \%$ & $100 \%$ & $100 \%$ \\
\hline
\end{tabular}

\begin{tabular}{|c|c|c|c|c|c|c|}
\hline & Simulations & Tutorial & VR & ES & $\mathrm{D} \& \mathrm{P}$ & Total \\
\hline & 1,003 & 2,135 & 183 & 116 & 1,795 & 5,232 \\
\hline \multirow[t]{2}{*}{ Public } & $19 \%$ & $41 \%$ & $3 \%$ & $2 \%$ & $34 \%$ & $100 \%$ \\
\hline & 62 & 143 & 18 & 2 & 83 & 308 \\
\hline \multirow[t]{2}{*}{ Private } & $20 \%$ & $46 \%$ & $1 \%$ & $1 \%$ & $27 \%$ & $100 \%$ \\
\hline & 1,065 & 2,278 & 201 & 118 & 1,878 & 5,540 \\
\hline Total & $19 \%$ & $41 \%$ & $4 \%$ & $2 \%$ & $34 \%$ & $100 \%$ \\
\hline
\end{tabular}


Table C 63. Types of Software Used by Sample Schools based on School Size (N=109)

\begin{tabular}{|c|c|c|c|c|c|c|}
\hline & Simulations & Tutorial & VR & $\mathrm{ES}$ & $\mathrm{D} \& \mathrm{P}$ & Total \\
\hline \multirow{3}{*}{ Small } & 158 & 296 & 47 & 6 & 101 & 608 \\
\hline & $15 \%$ & $13 \%$ & $23 \%$ & $5 \%$ & $5 \%$ & $11 \%$ \\
\hline & 168 & 851 & 52 & 4 & 199 & 1,274 \\
\hline \multirow[t]{2}{*}{ Medium } & $16 \%$ & $37 \%$ & $26 \%$ & $3 \%$ & $11 \%$ & $23 \%$ \\
\hline & 739 & 1,131 & 102 & 108 & 1,578 & 3,658 \\
\hline \multirow[t]{2}{*}{ Large } & $68 \%$ & $30 \%$ & $51 \%$ & $92 \%$ & $84 \%$ & $66 \%$ \\
\hline & 1,063 & 2,278 & 201 & 118 & 1,878 & 5,540 \\
\hline Total & $100 \%$ & $100 \%$ & $100 \%$ & $100 \%$ & $100 \%$ & $100 \%$ \\
\hline
\end{tabular}

\begin{tabular}{|c|c|c|c|c|c|c|}
\hline & Simulations & Tutorial & VR & ES & $\mathrm{D} \& \mathrm{P}$ & Total \\
\hline \multirow{3}{*}{ Small } & 158 & 296 & 47 & 6 & 101 & 608 \\
\hline & $26 \%$ & $49 \%$ & $8 \%$ & $1 \%$ & $17 \%$ & $100 \%$ \\
\hline & 168 & 851 & 52 & 4 & 199 & 1,274 \\
\hline \multirow[t]{2}{*}{ Medium } & $13 \%$ & $67 \%$ & $4 \%$ & $0 \%$ & $16 \%$ & $100 \%$ \\
\hline & 739 & 1,131 & 102 & 108 & 1,578 & 3,658 \\
\hline \multirow[t]{2}{*}{ Large } & $20 \%$ & $31 \%$ & $3 \%$ & $3 \%$ & $43 \%$ & $100 \%$ \\
\hline & 1,063 & 2,278 & 201 & 118 & 1,878 & 5,540 \\
\hline Total & $19 \%$ & $41 \%$ & $4 \%$ & $2 \%$ & $34 \%$ & $100 \%$ \\
\hline
\end{tabular}


Table C 64. Types of Commercially Purchased and Customized Software Used by Sample Schools based on Type of School $(\mathrm{N}=109)$

\begin{tabular}{|c|c|c|c|c|}
\hline & Commercial & Customized & Teacher-authored & Total \\
\hline \multirow{3}{*}{ Bachelors } & 968 & 35 & 14 & 1,017 \\
\hline & $44 \%$ & $22 \%$ & $33 \%$ & $43 \%$ \\
\hline & 1,210 & 20 & 49 & 1,279 \\
\hline \multirow[t]{2}{*}{ Associate } & $55 \%$ & $78 \%$ & $66 \%$ & $56 \%$ \\
\hline & 16 & 0 & 0 & 16 \\
\hline \multirow[t]{2}{*}{ Diploma } & $1 \%$ & $0 \%$ & $0 \%$ & $1 \%$ \\
\hline & 2,194 & 55 & 63 & 2,312 \\
\hline \multirow[t]{3}{*}{ Total } & $100 \%$ & $100 \%$ & $100 \%$ & $100 \%$ \\
\hline & Commercial & Customized & Teacher-authored & Total \\
\hline & 968 & 35 & 14 & 1,017 \\
\hline \multirow[t]{2}{*}{ Bachelors } & $95 \%$ & $3 \%$ & $1 \%$ & $100 \%$ \\
\hline & 1,210 & 20 & 49 & 1,279 \\
\hline \multirow[t]{2}{*}{ Associate } & $95 \%$ & $2 \%$ & $4 \%$ & $100 \%$ \\
\hline & 16 & 0 & 0 & 16 \\
\hline \multirow[t]{2}{*}{ Diploma } & $100 \%$ & $0 \%$ & $0 \%$ & $100 \%$ \\
\hline & 2,194 & 55 & 63 & 2,312 \\
\hline Total & $95 \%$ & $2 \%$ & $3 \%$ & $100 \%$ \\
\hline
\end{tabular}


Table C 65 . Types of Commercially Purchased and Customized Software Used by Sample Schools based on Type of Institution ( $\mathrm{N}=109)$

\begin{tabular}{|c|c|c|c|c|}
\hline & Commercial & Customized & Teacher-authored & Total \\
\hline & 1,985 & 55 & 61 & 2,101 \\
\hline \multirow[t]{2}{*}{ Public } & $90 \%$ & $97 \%$ & $82 \%$ & $90 \%$ \\
\hline & 209 & 0 & 2 & 211 \\
\hline \multirow[t]{2}{*}{ Private } & $10 \%$ & $3 \%$ & $18 \%$ & $10 \%$ \\
\hline & 2,194 & 55 & 63 & 2,312 \\
\hline Total & $100 \%$ & $100 \%$ & $100 \%$ & $100 \%$ \\
\hline
\end{tabular}

\begin{tabular}{|c|c|c|c|c|}
\hline & Commercial & Customized & Teacher-authored & Total \\
\hline \multirow{3}{*}{ Public } & 1,985 & 55 & 23 & 2,101 \\
\hline & $94 \%$ & $3 \%$ & $3 \%$ & 1005 \\
\hline & 209 & 0 & 11 & 211 \\
\hline \multirow[t]{2}{*}{ Private } & $99 \%$ & $0 \%$ & $1 \%$ & $100 \%$ \\
\hline & 2,194 & 55 & 29 & 2,312 \\
\hline Total & $95 \%$ & $2 \%$ & $3 \%$ & $100 \%$ \\
\hline
\end{tabular}


Table C 66. Types of Commercially Purchased and Customized Software Used by Sample

Schools based on Sizes of Schools $(\mathrm{N}=109)$

\begin{tabular}{|c|c|c|c|c|}
\hline & Commercial & Customized & Teacher-authored & Total \\
\hline \multirow{3}{*}{ Small } & 510 & 2 & 23 & 535 \\
\hline & $23 \%$ & $4 \%$ & $4 \%$ & $23 \%$ \\
\hline & 1,067 & 0 & 11 & 1,078 \\
\hline \multirow[t]{2}{*}{ Medium } & $49 \%$ & $0 \%$ & $1 \%$ & $47 \%$ \\
\hline & 617 & 53 & 29 & 699 \\
\hline \multirow[t]{2}{*}{ Large } & $28 \%$ & $8 \%$ & $4 \%$ & $30 \%$ \\
\hline & 2,194 & 55 & 63 & 2,312 \\
\hline Total & $100 \%$ & $100 \%$ & $100 \%$ & $100 \%$ \\
\hline
\end{tabular}

\begin{tabular}{|c|c|c|c|c|}
\hline & Commercial & Customized & Teacher-authored & Total \\
\hline \multirow{3}{*}{ Small } & 510 & 2 & 23 & 535 \\
\hline & $95 \%$ & $0 \%$ & $4 \%$ & $100 \%$ \\
\hline & 1,067 & 0 & 11 & 1,078 \\
\hline \multirow[t]{2}{*}{ Medium } & $99 \%$ & $0 \%$ & $1 \%$ & $100 \%$ \\
\hline & 617 & 53 & 29 & 699 \\
\hline \multirow[t]{2}{*}{ Large } & $88 \%$ & $8 \%$ & $4 \%$ & $100 \%$ \\
\hline & 2,194 & 55 & 63 & 2,312 \\
\hline Total & $95 \%$ & $2 \%$ & $3 \%$ & $100 \%$ \\
\hline
\end{tabular}


Integration of CAI 462

Table C 67. Ratings of Peers' Effectiveness at Integration Five Types of Software by Type of

School: Measures of Central Tendency $(\mathrm{N}=109)$

Simulations

\begin{tabular}{llccc}
\hline & Means & SD & Median & Mode \\
\hline Bachelors & 1.53 & 1.52 & 3.00 & 3.00 \\
Associate & 2.38 & 1.33 & 3.00 & 3.00 \\
Diploma & 1.20 & 1.17 & 2.00 & 1.00
\end{tabular}

Tutorials

\begin{tabular}{lllll}
\hline Bachelors & 1.26 & 1.44 & 3.00 & 3.00 \\
Associate & 2.61 & 1.33 & 3.00 & 3.00 \\
Diploma & 2.00 & 1.26 & 3.00 & 3.00
\end{tabular}

Virtual Reality

\begin{tabular}{lllll}
\hline Bachelors & 0.98 & 1.25 & 2.50 & 3.00 \\
Associate & 1.50 & 1.24 & 2.00 & 1.00 \\
Diploma & 1.00 & 1.10 & 1.00 & 1.00
\end{tabular}

Expert Systems

\begin{tabular}{lllll}
\hline Bachelors & 0.65 & 0.94 & 2.00 & 2.00 \\
Associate & 1.23 & 1.09 & 2.00 & 2.00 \\
Diploma & 0.40 & 0.49 & 1.00 & 1.00
\end{tabular}

Drill-and-practice

\begin{tabular}{lllll}
\hline Bachelors & 1.44 & 1.63 & 3.00 & 3.00 \\
Associate & 2.25 & 1.37 & 3.00 & 3.00 \\
Diploma & 1.60 & 1.30 & 2.00 & 1.00
\end{tabular}


Table C 68. Ratings of Peers' Effectiveness at Integration Commercially Purchased and Inhouse Produced Software: Measures of Central Tendency $(\mathrm{N}=109)$

Commercially Purchased Software:

\begin{tabular}{lcccc}
\hline & Means & SD & Median & Mode \\
\hline Bachelors & 1.69 & 1.60 & 3.00 & 3.00 \\
Associate & 2.68 & 1.38 & 3.00 & 3.00 \\
Diploma & 1.60 & 1.41 & 2.00 & 1.00 \\
Customized Software: & & & & 1.00 \\
\hline Bachelors & 0.82 & 1.10 & 2.00 & 2.00 \\
Associate & 1.24 & 1.20 & 2.00 & 0.00 \\
Diploma & 0.20 & 0.40 & 0.00 & \\
Teacher-authored Software: & 1.04 & 1.25 & & 3.00 \\
\hline Bachelors & 1.32 & 1.19 & 2.00 & 2.00 \\
Associate & 0.20 & 0.40 & 0.00 & 0.00 \\
Diploma & & & 2.00 &
\end{tabular}




\section{Appendix D}

Participants Typed Comments in Response to Question 23

\section{Comments From Non-CAI-users}

\section{A. Comments About How CAI is Used}

1. We have older computers that the student [sic] use with Internet access but no real CAI programs.

2. Our library has varied use capabilities for their computers. Some has complete use and others serve as a "card file"to strictly look up books, etc. like the old card file.

3. We make extensive use of fully web-based courses.

4. I teach mainly in the RN to BSN/MSN and MSN programs here. There is more CAI usage in the 'generic' BSN program.

5. I use a CAI for a non clinical-decision making activity. I use a CD-ROM Interactive Self Instructional Module for an Orientation to the RN Options Program.

6. We teach many web-based classes so having educational materials available on-line is an issue. Students also prefer to have access to programs via the web. But vendors do not have the products available yet or they are very expensive.

7. Faculty in the Department of Nursing use CAI's for instructional purposes however, I am unaware of faculty who use it for "Clinical Decision Making" as you define it. 
8. We are currently in the process of implementing a new pre-licensor undergraduate program. We do not yet have our first graduating class. Everything is under development at this time. We do a lot of web-based teaching, web-enhanced and web-assisted instruction. We currently do not have a nursing skills lab (under construction) but have been working with Cerner Corp to incorporate a comprehensive and progressive technology component for simulated clinical instruction.

\section{B Personal Comments}

1. I have used the CAI system at community college in the past. I am very familiar with teaching this system for teaching nursing students. I taught at Clovis Community College from 1994-2000 using this system.

2. I do plan on using CAI in the future however I am a new faculty member and have not had time to really find any CAI to integrate into the curriculum. I have integrated the Internet.

Comments About the School

I. We have both an Associates and Bachelors Program.

II. Comments of CAI-users

\section{A. Comments About How CAI is Used}

1. I am not sure that we use computers in the way you are looking for in this study. We use computer software (such as case studies) powerpoints [sic] 
and web sites to teach the classroom content and some lab content. We do not use it for clinical procedure checkoffs and competencies. The case studies are used more for discussion and either presentation or review of material. The students make decisions as they progress through the case studies but the decisions are incorporated into learning the material rather than mastery of the content. Certain CDs are available for the students to access at any time in the general computer lab. Other CDs are kept by individual instructors and they can be set up on laptops in the nursing lab for the students to use. We are trying to incorporate computers into our nursing lab. It is scheduled for renovation and a computer station is on our "wish list". We are also under budget restraints because we are supported by the state which like many other states has had monetary deficits the last few years. Good luck with your thesis and feel free to contact me for clarification.

2. Limited University Resources- Nursing faculty serve as Instructional Designers and need to maintain CAI independently and development. Student numbers reflect upper division major - junior year

3. Computer resources on our campus are located in departments as well as shared locations such as computer center library and union. Item 17 is difficult to answer; we do not have a "school of nursing" but a college of health sciences. While we have a computer lab in the school it is not dedicated to health sciences $[$ sic $]$ students but shared with students across campus. We do not have any personnel on-site staffing this lab; it is 
managed by a central IT service. These facts make response to some of your items difficult and perhaps misleading.

4. I use a Web CT hybrid approach to teach an Accelerated ADN Program. Students can attend classes for four semesters (including the summer) and complete the ADN in 18 months as opposed to two years for the Traditional ADN Program. All faculty (traditional and accelerated) use CAI to teach clinical decision making skills. Since the accelerated program uses the WebCT format in the hybrid approach we rely heavily on CAI for assistance in teaching clinical decision making. Time is very valuable so the hybrid approach offers our adult learners options for enhancing their learning and their skills. We have links to Internet sites that reinforce the lessons and don't hesitate to use the CAI programs that come with our textbooks.

5. We use LSRN and LSPN and also interactive videos. We do not have any of the real sophisticated CAI.

6. We have purchased a CAI program but have not had a workshop to explore it and see how to use it to teach clinical decision making. I would love to know the results of your study when complete. Good Luck.

7. We have all used CAIs at this school of nursing (6 instructors). In fact, my project for my MSN was to design a CAI for the nursing program. The cost of continually updating the CAIs every time the technology department updated computers (approximately every 2 years) was prohibitive for our program. We have pulled our CAI resources and 
kept/updated only those that are most recent in content which happened to be those in the Psychiatric Nursing area. We are currently looking at online CAI instruction rather than buying software. I'm sure you appreciate the woes of our state budget like so many others. We just added a cost to the students every semester for online testing and would not be able to pass a CAI cost to the student at this time. We used it as an alternative way to present material especially for students with different learning styles and to increase critical judgment. Good luck with your study.

8. I do not test students for their ability to use CAI because they have to pass a battery of test to be accepted to the nursing program.

9. Personally, have found it very useful to assign highly related simulation programs as weekly clinical preparation for my Maternal-Newborn Nursing Course (i.e. students complete programs related to Labor and Delivery before starting their clinical experience in labor and delivery; postpartum programs before starting postpartum; prenatal programs before attending the prenatal clinic etc.

10. CAIs are mainly used in our institution as a supplemental materials to the course, as an evaluative tool in competencies and is also very helpful in making up lost work in either clinical or classroom setting. I can also duplicate multiple copies for students to each have their own copy.

This increases their flexibility on when they are able to use the software. The reason I am able to create the interactive software is that we have 
excellent faculty support for computer skills and development. We have an entire department that is here just to support teaching. The instruction they provide for faculty includes all types of different computer programs as well as teaching strategy seminars. They provide video and audio equipment for loan and assist us in learning the newest computer technology. We also have computer support for the faculty as well as the student body and they will come to our offices and help us with problems.

11. Faculty at all levels of Nursing education need to increase awareness \& usage of computerized educational programs for students. Allows for a diversity of learning experiences to be explored by the students. Too many old time faculty members do not want change even if it is for the betterment of the students.

12. We just recently (within 6 months) began using simulation technology here at the college. The information I have provided is based, in part, on my observations over the past six months. Interestingly, as the faculty become more comfortable with the technology and begin to see that student learning can be enhanced through these types of programs I am sure that they will experience an increase in personal time that can be devoted to other educational activities. Also with regard to question 13 we have 160 undergrads and approximately 30 graduate students presently in the program...I wasn't sure if you only wanted undergrad numbers. Thank you for the opportunity to participate in your study.

13. I feel CIA helps the students to visualize and apply the theory we cover in 
Integration of CAI 470

class. I would like to see more virtual programing for Critical thinking on an introductory level for 1st year nursing students.

14. Faculty at this school seem resistant to new technology and teach in the same way they have learned and in the same way they have taught in the past. We also have a lack of technical support.

15. Students are often much more interested in computer instruction than faculty. The learning curve for faculty to use technology remains a problem. Faculty using technology spend significantly more time in developing/monitoring technology in courses. Commercially prepared CAI have not fully embraced the depth of knowledge needed. They tend to focus on 'knowledge/comprehension' with some 'application' and no 'synthesis'......

16. A lot of what was asked in the survey is dependent on money -- what we would like to do and what we are able to utilize are two entirely different categories (sometimes I wasn't sure which way to go with the answer)

17. We are moving to more integrated computer use. We hope to have our first computer online course this fall.

18. I use Educator as an adjunct to my lecture. I teach nursing 105-physical assessment. I use this LMS to post lecture notes links to sites that will help them with physical assessment practice tests grades

19. It is very difficult to get faculty to use CAI but we do use it when we can. We do have several computer mannequins that do require students to 
apply critical thinking and decision making when working with these mannequins in small lab group settings. These mannequins are only used in 2-4 lab sessions / semester but as faculty learn more then students are more exposed to the CAI in these situations.

20. I am relatively unsure about the extent that other faculty members use CAI in our school. For one class in med-surg that I co-teach my partner uses a CAI program on hemodynamics. I actually do not require CAI at all. I do tell students about some Internet sites with self-paced learning CAI activities but that is all. Most of my computer based activities are related to the traditional classroom and on-line enhanced pharm course I teach. (P.S. I am so sorry this is late. I don't recall receiving it before.) Also your on-line survey tool is exceptionally well done! Cindy

21. Some of the questions were impossible to answer because the answer was not available - for instance, I go over the CAI info each time it is used some semesters that is weekly some monthly some not at all. I don't think cost is an issue - if anything - buying and using the software is more expensive because I do not use it to replace me in the classroom (even if I did - the school would still have to pay me!) I use them to supplement and to help those students for whom self paced learning is more helpful. We also use them as clinical simulations and then discuss and use the info at the next clinical time. I truly hope you are not finding schools who use CAI's to decrease the time they spend with students!! 


\section{B. Personal Comments}

1. I'm currently working to complete my MSN (May 2005!).

2. MSN completed December 11, 2003 -Tulsa Community College13 hours left before have MSN

3. It was difficult to answer some of the questions if I had not used the CAI in the format you asked. I have a master's in nursing and education but the computer would not let me enter both.

C. Comments about the School

1. We are an articulated nursing program with both ASN and BSN. I was not able to indicate that using the radio buttons. We use CAI in both programs so I put down BSN.

2. CAI installed in the university's server. The university provides computer support. I don't know exact number of staff is available.

3. Development of a hybrid nursing program is currently underway.

D. Comments about Answers

1. Would have liked an "UNSURE" choice for questions that were not really able to be accurately answered.[sic]

2. The one place I didn't put in numbers was the part about the computer lab support.

3 I have no idea. There are three nursing faculty who support the computers in the nursing skills lab. 
4. I could not answer how many full and part time employees work in the University's [sic] computer lab. These labs are also used to support our students. Also on questions 8 and 10 I put Strongly Disagree but they were really not applicable because we do not have our own made CAI's. I just didn't have another option answer.

5. I was not able to answer questions that asked for specific numbers because there is no aggregated data available related to use of software. A number of zero is not always accurate for questions. 


\title{
Curriculum Vitae
}

Beverly J. Schaefer R.N., M.S.N.

10242 Lola Court

Concord, $\mathrm{OH} 44077$
Phone: 440-350-7229

Schaefer440@sbcglobal.net

\section{ACADEMIC APPOINTMENTS:}

1997- 2000

\author{
Meridia Huron School of Nursing-Cleveland Clinic \\ Health Systems \\ East Cleveland, $\mathrm{OH}$ \\ Instructor and Course Co-ordinator
}

Full-time position as clinical, didactic instructor and course co-ordinator for senior level courses. Clinical areas of teaching included ICU, CCU, acute and chronic pediatrics units at Metro-Health Medical Center, adult medical-surgical, ED, and co-ordinated and supervised students during the final semester preceptor experience. Served as Chair of the A-V Committee, member of Curriculum Committee, Curriculum Revision, Evaluation Committee, and Faculty Committee.

1991- 1993

\author{
West Virginia University School of Nursing \\ Morgantown, WV \\ Visiting Professor
}

Full-time position as medical-surgical lecturer and clinical instructor. Supervised junior nursing students on a medical-surgical trauma nursing unit of West Virginia University- Ruby Memorial Hospital. Shared responsibilities for lectures and weekly discussion groups for the junior level "Issues and Trends in Nursing Class." Other duties included student advising, Junior Class Faculty Advisor, Faculty Development Committee. 
1989- 1991

\author{
West Virginia Northern Community College - School \\ of Nursing \\ Weirton and Wheeling, WV \\ Lecturer and Clinical Instructor
}

\begin{abstract}
Course co-ordinator and sole lecturer for a "Fundamentals in Nursing Course" for three semesters and co-lectured for two semesters of "Simple Health Problems."

Supervised clinical experiences for students on pediatric and adult medical-surgical nursing units. Supervised students for one semester on a psychiatric mental health in-patient facility on the Wheeling campus. Served as academic advisor to 40 students, faculty advisor to the Student Nurses Association and mentored several minority students who participated in the school's "Faculty- Mentor Program." Served on the Curriculum Revision Committee for the School of Nursing and National League of Nursing Accreditation Self-Study Committee. Oriented new faculty to the Weirton campus and the teaching role and responsibilities.
\end{abstract}

\title{
PROFESSIONAL EXPERIENCE:
}

1993- 1999

\author{
West Virginia University Hospital \\ Morgantown, WV \\ Staff-Nurse Adult Trauma Unit
}

Full-time staff- nurse on the Medical-Surgical, Trauma Adult Care Nursing Unit at Ruby Memorial Hospital. Other services on the unit included Plastic Surgery, ENT, and Surgical Oncology. Duties included direct patient care and supervision of other nurses on the unit and in a step-down ICU.

1989- 1993

\author{
West Virginia University Hospital \\ Morgantown, WV \\ In-Service Nurse Educator
}

Temporary part-time position as an independent contractor working with the nursing education department. Responsibilities included teaching and evaluating graduate nurses and preceptors on adult acute care nursing units of Ruby Memorial Hospital, writing weekly reports on the graduates' progress and adjustment to the R.N. role. 
1989- 1991

\author{
Wheeling Hospital \\ Wheeling, WV \\ Neuro-Vascular Technologist and Staff-nurse
}

Worked in the neuro-vascular laboratory- full-time from January until August 1989. Responsibilities included taking patient histories, conducting physical examinations, performing carotid artery duplex scans, OPG testing, EMGs and EEGs. Held a staff-nurse position full time from August 1989 until January 1990 and then part-time until March 1991 while teaching at WVNCC. Floated between various departments which included an intermediate coronary care unit, GI unit, medical-surgical, oncology, gynecology and orthopedics nursing units. Responsibilities were numerous and varied by unit.

1988

Vascular Technical Services, Inc.

Pittsburgh, PA

Non-invasive Peripheral Vascular Technologist-RN

Independent contractor working under contract with five different clinics, physicians' offices and hospitals in the Pittsburgh and Uniontown area. Tests included venous and arterial pressure measurements for the detection of peripheral vascular diseases and deep vein thrombosis. Duties included conducting studies, interpreting the results and writing reports.

1985- 1988

\author{
East Ohio Regional Hospital \\ Martins Ferry, $\mathrm{OH}$ \\ Non-invasive Peripheral Vascular \\ Technologist-RN and Behavior Health Nurse
}

Shared time equally between the non-invasive peripheral vascular laboratory and the drug and alcohol rehabilitation "CARE Unit" from 1996 until 1988. In the laboratory, performed duplex scans of the carotid arteries, arterial and venous imagining and peripheral vascular pressure readings. Cross-trained in the echo-cardiograph laboratory. In the CARE Unit, supervised other nurses and worked collaboratively with physicians and counselors in providing care for twenty in-patients and their families. Duties included supervising and providing nursing care during the acute phases of treatment, leading daily group discussions, organizing "Interventions" with employers and family members, and teaching classes for groups of clients and families. Served as the Care Unit Alumni Program Coordinator. 
1983- 1989

\author{
Harrison Community Hospital \\ Cadiz, $\mathrm{OH}$ \\ Staff nurse
}

Employed full time during the summer and then part-time on week-ends while attending undergraduate classes at West Liberty State College. Supervised LPNs, and aids in the direct care of patients on a medical surgical unit.

\title{
EDUCATIONAL PREPARATION:
}

1993- 2005

\author{
Doctorate in Technology Education \\ Department of Advanced Educational Studies \\ West Virginia University
}

Majored in the communications branch of the School of Technology Education and minored in "Computers in Nursing Education." Majority of courses were in the area of instructional design, educational theories, the use of technology delivery systems for adult education, Internet applications and research, and the use of multimedia for higher education. Other courses included the Philosophy of Technology and Society, numerous courses in the history of technology, communications, distance education, and educational measurements.

1995- 1988

\author{
Master of Science in Nursing \\ West Virginia University \\ Morgantown, WV
}

Prepared as an advanced primary nurse practitioner in assessing, planning and implementing nursing interventions with adults, families and groups. A large part of my course work was in the research process, nursing theories, and implementing Bowens' Family Systems Theory with families in a community health setting. 


\author{
Bachelor of Science in Nursing \\ West Liberty State College \\ West Liberty, WV
}

Graduated with "High Honors," and participated in the Honor's Program. Received the "Outstanding Community Health Nurse Award" and was named to the "Dean's List" and listed in "Who's Who in American Colleges" each semester. Focus of course work, in addition to nursing, was in advanced sciences. Served as student representative to the N.L.N. Self-Study for Accreditation of the B.S.N. program from 1983 until 1985.

1980- 1983
Associate Degree in Nursing Science
West Virginia Northern Community College
Wheeling, WV

Named to the "Dean's List," the "Presidents List," and "Who's Who in American Colleges" all but the first semester.

\title{
PROFESSIONAL PRESENTATIONS:
}

1998

\author{
Faculty Development Workshop \\ Introducing Technology in the Classroom
}

Presented a workshop for the faculty of the Meridia Huron School of Nursing on the use of "Authorware and Power-Point Presentation Software" to support multimedia slide presentations for lectures and conference presentations. Presentation included a special focus on the strategies of good instructional design and teaching practices with computer assisted-instruction.

$$
\begin{aligned}
& \text { Orthopedic Workshop } \\
& \text { West Virginia University SON }
\end{aligned}
$$

Worked collaboratively with two other faculty members to design the curriculum and develop instruction for a two-day workshop for the school of nursing faculty and the junior nursing class. 
Worked collaboratively with other members of the Ad-Hoc Task Force in planning a three-day conference. Acted as group leader and recorder to assist the seventy nurses who attended the conference in the development of a plan of action to improve the timing and quality perinatal health care for women and children of Appalachian West Virginia. The compiled report was presented to Representative Robert White and Senator J. Rockefeller, who were both in attendance and has been since included in both Federal and State legislative health care reform plans and legislation.

1989

Wheeling Jesuit College

Wheeling, WV

Guest-speaker for a co-operative intensive care course presented to Wheeling Hospital on "Nursing Care and Assessment of the Client with Peripheral Vascular and Venous Disorders."

\section{CLASSES AND WORKSHOPS ATTENDED:}

Presentation of a study conducted by a nurse researcher who spent four days on the streets of New York disguised as a homeless person in order to document the quality of the health care being provided by the health care resources that are available to the homeless. 
Informatics and Other Uses of Computers in Nursing

Breen School of Nursing, Ursuline College

Pepper Pike, $\mathrm{OH}$

Presentation by Dr. Leslie Nicoll the Editor-in-Chief of Computers in Nursing Journal.

1998

Conference on Critical Thinking \& Clinical

Decision Making in Nursing Education

Columbus, $\mathrm{OH}$

Attended a conference presented by Mount Carmel Hospital to define critical thinking and clinical decision-making and how the concepts are related to nursing education. The focus was on why the instruments that are currently used to assess critical thinking relate so poorly to the outcomes of graduates on the State Board examinations.

1993

National NCLEX-CAT Conference

Atlanta, GA.

Attended as the West Virginia University SON representative to learn about State Board Test construction and the implementation of Computer Adaptive Testing format.

"Assessment: Are Your Student's Learning What You Are Teaching?"

West Virginia University Faculty Development Morgantown, WV

Presented by Dr. P. Cross from the Second National Conference of Teachers. 


\section{Morgantown, WV}

Attended a three-day workshop presented by Linda Brader from the Computer Based Educational League - Teaching and Learning Technologies Center of Academic Computing of Penn State.

1992

NIH Clinical Center Nursing Presentation and Tour of the Facilities.

Bethesda, MD

Accompanied a group of twenty WVU students to the National Institute of Health in Bethesda, Maryland facilities as the Junior Class Advisor.

1991

National Committee for Nursing Research

Conference on Health Promotion for Rural

Communities

Morgantown, WV

A three-day conference that was sponsored by the Alpha Rho Chapter of Sigma Theta Tau where several nationally known speakers presented topics and research findings and their implications for rural health nursing.

1991

1991

1991
Determining the Patient's Right to Decide: Ethics Teleconference

Charleston Area Medical Center

Charleston, WV

Teaching Effectively

WVU Faculty Development

Morgantown, WV

Teaching with SATNET

WVU School of Nursing Faculty Development

Morgantown, WV 
1990

1990

1988- 1989
Cognitive Theories in Education Seminar

Pittsburgh Area Conference Schools of Higher Education, Pittsburgh, PA

The Use of Computers in Education Seminar

IBM Corporation, Pittsburgh, PA

Coronary Care Course

Wheeling Hospital In-service Offering

Wheeling, WV

\section{RESEARCH:}

1993 “Student's Responses to Computerized Testing." An on-line computer testing format was compared with pencil and paper testing from February until May 1993. Unpublished.

1988 "The Impact of an Aerobic Walking Program on the Participation of Persons with Atherosclerosis Obliterans of the Lower Extremities." West Virginia University Masters' Paper. The study explored the use of social support as a motivator to encourage persons with peripheral vascular disease to participate in an exercise program.

1985 "A Summative Study of the Perceptions of Mastery of Professional Skills and Influence on Conceptual Framework of Graduates of a Two-Plus-Two BSN Program.” Responses to a mailed questionnaire were compiled from seventy former West Liberty State College Nursing Students and their employers to determine the effectiveness of the program in meeting its program objectives. The study was conducted as part of a requirement for participation in the WSLC Honor's Program and was presented to the Honor's Program participants, faculty, and advisors. Unpublished. 


\section{PROFESSIONAL MEMBERSHIP:}

1988- 2000

Sigma Theta Tau International Nursing Honor Society- Alpha Rho Chapter

Morgantown, WV

Active membership which included participation in the recommendation and selection of new members and attendance at meetings and conferences whenever possible.

2000-2001

Sigma Theta Tau International Nursing Honor Society- Nu Delta Chapter

Cleveland, $\mathrm{OH}$

Actively participated in organization through attendance at meetings and conferences.

\section{PUBLIC SERVICE:}

2003- Present

CARE Ministry of the Willoughby Hills Evangelical Friends Church

Willoughby Hills, $\mathrm{OH}$

Duties include meeting and greeting new attenders and following up with phone calls and assistance during their first months of attendance at the church.

1990- 1993

Perinatal Action Group for the Health Care of Women and Children in Appalachian West Virginia

A working group that was a division of the Association of Deans and Directors of Nursing Education in West Virginia. Attended monthly meetings in Charleston and Flatwoods, WV. Planned a statewide conference and CEU courses for nurses who attended a three day multi-state conference in Charleston. The group served as a spring board for major legislative changes such as mandatory CEU's for RN licence renewal, gaining prescription writing privileges for advance practitioners and calling attention to the crisis situation and the lack of adequate health care in the state for pregnant women. 
Organized nursing students in planning a huge health fair for the WeirtonStubenville area residents and the students and faculty of WVNCC.

1986- 1989

Advisor to the Care Unit Alumni Association

East Ohio Regional Hospital

Martins Ferry, $\mathrm{OH}$

As part of my employment and in order to carry out various community level projects in the masters' level courses, I organized and taught courses for alumni and family members, some of which included: Communications Skills with the Family, Assertiveness vs. Aggression, Problem-Solving Strategies, Healthy Living and Nutrition, Family Dynamics and the Therapeutic Use of Humor. Also planned and supervised the group's many social activities.

1983- 1986

Crisis Hotline Volunteer

Wheeling, WV

Volunteer duties included six hours each week of answering crisis calls, providing appropriate interventions and making referrals to various community agencies for follow-up assistance including the Rape and Domestic Violence Center and counseling services with the financial and mental health facilities in the Wheeling, WV area. 